\title{
Lachgas- und Methanflüsse eines Gley-Auenbodens unter dem Einfluß einer Rapsfruchtfolge und in Abhängigkeit von der N-Düngung
}

\author{
Dissertation \\ zur Erlangung des Doktorgrades \\ der Fakultät für Agrarwissenschaften \\ der Georg-August-Universität Göttingen
}

vorgelegt von

Frank Schmädeke

geboren in Nienburg/Weser 
D 7

1. Referent

Prof. Dr. E. Przemeck

2. Korreferent

Prof. Dr. F. Beese

Tag der mündlichen Prüfung:

05. November 1998 
Diese Arbeit wurde mit Mitteln der DeUtsche BundesstifTung UMWELT im Rahmen des Verbundprojektes

„klimarelevante Spurengasflüsse beim Anbau nachwachsender Rohstoffe“ gefördert 


\section{Inhaltsverzeichnis}

Seite

1

Einleitung.............................................................. 1

$2 \quad$ Literaturübersicht............................................... 5

$2.1 \quad \mathrm{~N}_{2} \mathrm{O}$-Bildung in Böden..................................................... 5

2.1.1 Ökologische Bedeutung 5

2.1.2 Prozesse der $\mathrm{N}_{2} \mathrm{O}$-Bildung in Böden 5

2.1.3 $\quad \mathrm{N}_{2} \mathrm{O}$-Emissionen aus landwirtschaftlich genutzten Flächen 13

$2.2 \quad \mathrm{CH}_{4}$-Bildung und -Verbrauch in Böden................................ 15

$\begin{array}{lll}2.2 .1 & \text { Ökologische Bedeutung } & 15\end{array}$

2.2.2 Prozesse der Oxidation atmosphärischen Methans in Böden 17

$\begin{array}{lll}\text { 2.2.3 } & \mathrm{CH}_{4} \text {-Aufnahme terrestrischer Böden } & 18\end{array}$

3 Über die Bedeutung des Winterrapsanbaus und der $\mathrm{N}$-Düngung für die $\mathrm{N}_{2} \mathrm{O}$-und $\mathrm{CH}_{4}$-Flüsse eines Gley-Auenbodens

3.1

Einleitung..................................................................... 21

3.2 Material und Methoden..................................................... 24

$\begin{array}{lll}3.2 .1 & \text { Versuchsstandort } & 24\end{array}$

3.2.1.1 Geographische Lage, Klima und Witterung 24

3.2.1.2 Physikalische und chemische Eigenschaften des Bodens 26

$\begin{array}{lll}3.2 .2 & \text { Versuchsvarianten } & 27\end{array}$

$\begin{array}{lll}\text { 3.2.3 Untersuchungsmethoden } & 28\end{array}$

3.2.3.1 Gewinnung der Gasproben im Feld 28

$\begin{array}{lll}\text { 3.2.3.2 Spurengasanalytik } & 30\end{array}$

$\begin{array}{lll}\text { 3.2.3.3 Berechnung der Spurengasflüsse } & 30\end{array}$

3.2.3.4 Bodenphysikalische Untersuchungen 31

$\begin{array}{lll}\text { 3.2.3.4.1 Bodenwassergehalt } & 31\end{array}$

3.2.3.4.2 Lagerungsdichte und Gesamtporenvolumen 32

3.2.3.4.3 Volumetrischer Wassergehalt und volumetrischer Luftgehalt 32 
3.2.3.4.4 Anteil des volumetrischen Wassergehaltes am Gesamtporenvolumen des Bodens

3.2.3.4.5 Porengrößenverteilung und Korngrößenfraktionierung 33

3.2.3.5 Bodenchemische Methoden 33

3.2.3.5.1 pH-Wert 33

3.2.3.5.2 Gesamt-C- und Gesamt-N-Gehalte des Bodens 33

3.2.3.5.3 Carbonat-C 34

3.2.3.5.4 Mineralischer Stickstoff 34

3.2.4 Bewertung der Treibhauswirksamkeit 34

$\begin{array}{lll}3.2 .5 & \text { Statistische Auswertung } & 37\end{array}$

3.3 Ergebnisse............................................................... 38

3.3.1 Zeitlicher Verlauf der Bodenparameter 38

3.3.1.1 Bodentemperatur und wassergefüllte Porenvolumina 38

3.3.1.2 Ammonium- und Nitratgehalte des Bodens 42

3.3.2 Verlauf der $\mathrm{N}_{2} \mathrm{O}$-Emissionen 46

3.3.3 Einflüsse der Bodenparameter auf die $\mathrm{N}_{2} \mathrm{O}$-Emissionen der verschiedenen Fruchtarten $\quad 50$

3.3.4 Kumulierte $\mathrm{N}_{2} \mathrm{O}$-Emissionen 52

3.3.5 Verlauf der $\mathrm{CH}_{4}$-Flüsse 55

3.3.6 Einflüsse der Bodenparameter auf die $\mathrm{CH}_{4}$-Flüsse 55

3.3.7 Kumulierte Methanflüsse 59

3.4 Diskussion...................................................................... 60

3.4.1 $\mathrm{N}_{2} \mathrm{O}$-Emissionen des Bodens $\quad 60$

3.4.2 $\mathrm{CH}_{4}$-Aufnahme des Bodens 68

3.4.3 Klimarelevanz der Produktion der unterschiedlichen Fruchtarten 73 


\section{Untersuchungen über die $\mathrm{N}_{2} \mathrm{O}$ - und $\mathrm{N}_{2}$-Emissionen in unterschiedlichen Tiefen eines Gley-Auenbodens im südniedesächsischen Leinetal}

4.2.1 Entnahme von ungestörten Bodensäulen 80

4.2.2 Funktion und Betrieb der Mikrokosmenanlage 80

4.2.3 Versuchsvarianten 82

4.2.4 Berechnung der Spurengasflüsse in den Mikrokosmen 83

4.2.5 pF-Charakteristik der Bodenhorizonte 83

4.2.6 Physikalische Eigenschaften der Bodensäulen 84

4.2.7 Bestimmung der Denitrifikation Hilfe der ${ }^{15} \mathrm{~N}$ Gasfreisetzungs-Methode $\quad 86$

4.2.7.1 Das Inkubationssystem 86

$\begin{array}{ll}\text { 4.2.7.2 Entnahme und Aufbereitung der Gasproben } & \\ \text { für die Isotopenanalyse } & 87\end{array}$

$\begin{array}{lll}\text { 4.2.7.3 } & { }^{15} \mathrm{~N} \text {-Analytik } & 87\end{array}$

4.2.7.4 Berechnung der denitrifikativen $\mathrm{N}_{2}$-Freisetzung 89

$\begin{array}{lll}4.2 .8 & \text { Statistische Auswertung } & 90\end{array}$

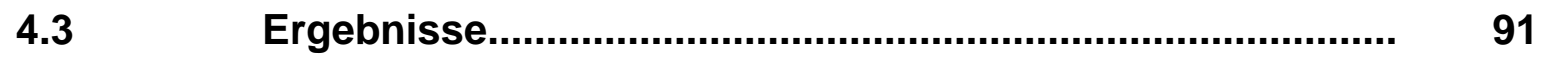

4.3.1 Transporteigenschaften der Bodensäulen für Wasser 91

4.3.2 Nitrataustrag mit dem Perkolat 94

$\begin{array}{lll}\text { 4.3.3 } & \mathrm{N}_{2} \mathrm{O}-\text { Flußraten } & 96\end{array}$

$\begin{array}{lll}\text { 4.3.4 } & \mathrm{CO}_{2} \text {-Flußraten } & 98\end{array}$

4.3.5 $\mathrm{N}_{2}$-Emissionen $\quad 99$

$4.4 \quad$ Diskussion ............................................................................

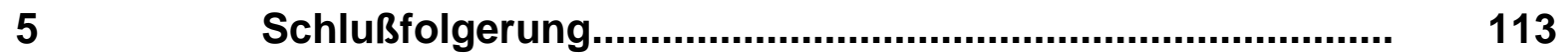

$6 \quad$ Zusammenfassung .................................................... 120

$7 \quad$ Literaturverzeichnis......................................................... 123 
Verzeichnis häufig verwendeter Abkürzungen

Abb.

AMO

C

$\mathrm{CH}_{4}$

Corg

$\mathrm{CO}_{2}$

$d_{B}$

ECD

FCKW

FID

$\mathrm{GC}$

GOF

GPV

GWP

i.d.R.

IPCC

Kap.

KAS

$\mathrm{MMO}$

$\mathrm{N}$

$\mathrm{N}_{\min }$

NO

$\mathrm{NO}_{3}$

$\mathrm{N}_{\mathrm{t}}$

$\mathrm{N}_{2} \mathrm{O}$

$\mathrm{N}_{2} \mathrm{O}_{\text {Feld }}$

$\mathrm{N}_{2} \mathrm{O}_{\text {ind }}$

$\mathrm{O}_{2}$

$\mathrm{PV}$

$r$

$r^{2}$

RME
Abbildung

Ammoniummonooxygenase

Kohlenstoff

Methan

organisch gebundener Kohlenstoff

Kohlendioxid

Lagerungsdichte des Bodens

electron capture detector

Fluorchlorkohlenwasserstoff

flame ionisation detector

Gaschromatograph

Geländeoberfläche

Gesamtporenvolumen des Bodens

global warming potential

in der Regel

Intergovernmental Panel on Climate Change

Kapitel

Kalkammonsalpeter

Methanmonooxygenase

Stickstoff

mineralischer Stickstoff

Stickstoffmonoxid

Nitrat

Gesamtstickstoff

Distickstoffoxid (Lachgas)

$\mathrm{N}_{2} \mathrm{O}$-Feldemissionen

indirekte $\mathrm{N}_{2} \mathrm{O}$-Emissionen

Sauerstoff

Porenwasservolumen der Bodensäulen

Korrelationskoeffizient

Bestimmtheitsmaß

Rapsölmethylester 


$\begin{array}{ll}\text { S } & \text { Sand } \\ \text { T } & \text { Ton } \\ \text { Tab. } & \text { Tabelle } \\ \text { Temp. } & \text { Temperatur } \\ \text { TM } & \text { Trockenmasse } \\ \text { Tg } & \text { Teragramm } \\ \text { U } & \text { Schluff } \\ \text { UBA } & \text { Umweltbundesamt } \\ \text { Vk } & \text { Variationskoeffizient } \\ \text { Vol.H2O } & \text { volumetrischer Wassergehalt des Bodens } \\ \text { vgl. } & \text { vergleiche } \\ \text { WFPS } & \text { water-filled pore space (wassergefülltes Porenvolumen) } \\ \text { WGerste, WG, G } & \text { Wintergerste } \\ \text { WRaps, WRa, R } & \text { Winterraps } \\ \text { WWeizen, WW, W } & \text { Winterweizen }\end{array}$


Seit Beginn der Industrialisierung Anfang des 19. Jahrhunderts ist in der Erdatmosphäre die natürliche Konzentration von klimawirksamen Gasen wie Kohlendioxid, Stickstoffoxiden $\left(\mathrm{N}_{2} \mathrm{O}, \mathrm{NO}\right.$ und $\left.\mathrm{NO}_{2}\right)$, Methan und FCKW stark angestiegen. Als Hauptverursacher hierfür gelten die Verbrennung fossilen Brennstoffs im Energie- und Verkehrssektor, die landwirtschaftliche Inkulturnahme bisher unberührter Naturräume, landwirtschaftliche Bewirtschaftungsmaßnahmen, sowie die weltweite Produktion von FCKW (Abb. 1-1). Insgesamt trägt das klimarelevante Spurengas Stickstoffdioxid zu ca. 6 \% zum anthropogen bedingten Treibhauseffekt bei. Für Methan macht dieser Anteil sogar ca. $19 \%$ aus (BouWMAN, 1990; IPCC, 1994; IPCC, 1995).

\section{Energieverbrauch}

(Energie- und Verkehrssektor)

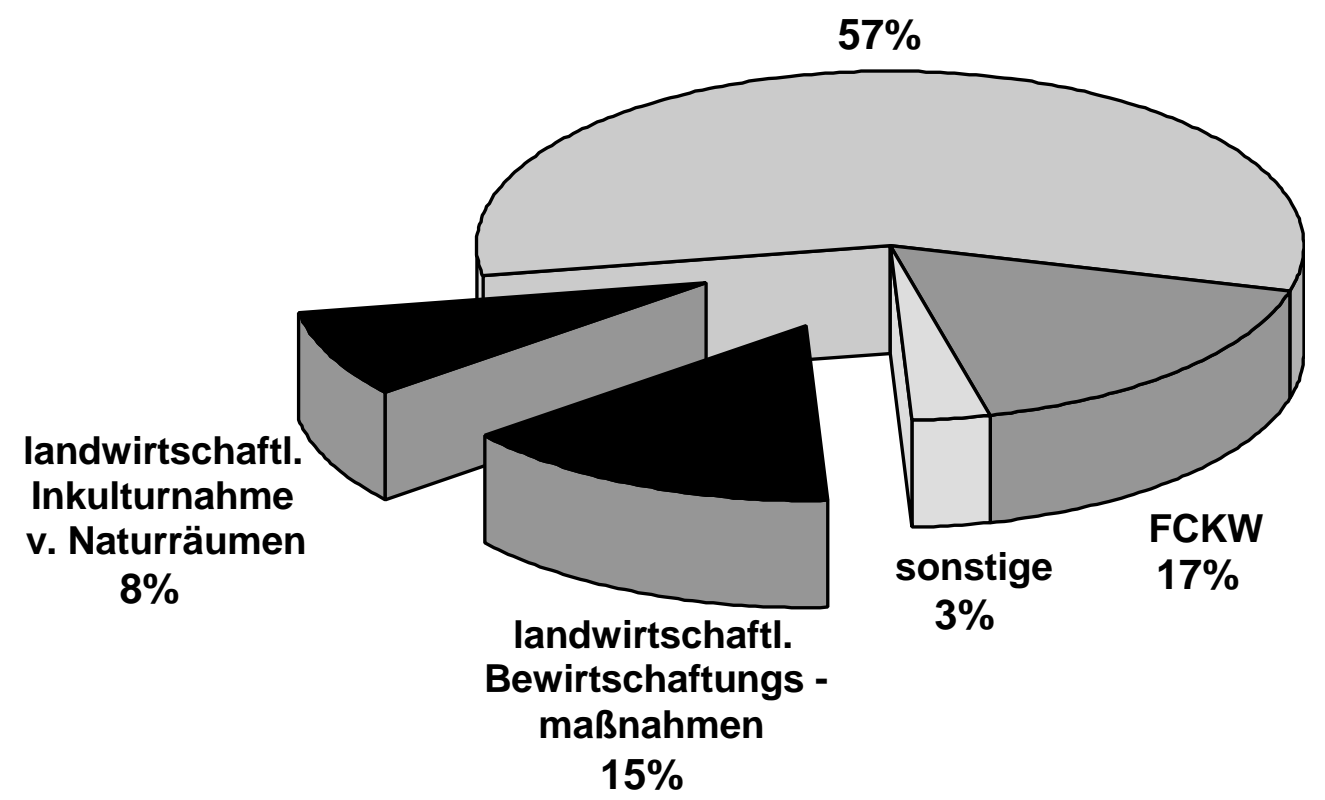

Abb. 1-1: Beiträge anthropogener Aktivitäten zum Treibhauseffekt (nach USEPA, 1989).

Die anthropogen bedingte Belastung der Erdatmosphäre mit Spurengasen stellt aufgrund der damit verbundenen Erwärmung der Atmosphäre und der Zerstörung der Ozonschicht ein ökotoxikologisches Problem dar. Da die Emission klimarelevanter Gase bei der Verbrennung fossiler Energieträger als Hauptverursacher des globalen Treibhauseffektes gilt, wird auch international über 
die Einschränkung ihrer Nutzung diskutiert und u.a. die Substitution fossiler Rohstoffe durch die Produktion nachwachsender Rohstoffe vorgeschlagen.

Bei nachwachsenden Rohstoffen handelt es sich um regenerative Energieträger, für deren Produktion in der EG aufgrund der derzeitigen landwirtschaftlichen Überproduktion Flächen zur Verfügung stehen. Nachwachsende Rohstoffe wie z.B. der Raps zur Produktion von Rapsölmethylester (RME) als Ersatz von Dieselkraftstoff gelten als klimaverträglich. Im Gutachten des Umweltbundesamtes wird jedoch darauf hingewiesen, daß sowohl die Produktion als auch der Verbrauch nachwachsender Rohstoffe nicht frei von Umweltbelastungen und klimawirksamen Emissionen ist (UBA, 1993). Die häufig angeführte Klimaneutralität der Nutzung nachwachsender Rohstoffe sei nicht zwangsläufig gegeben. So sei es nicht sichergestellt, daß die Bereitstellung von RME als Substitut für Dieselkraftstoff eine Umweltentlastung bewirke. Es müssen die Umweltbelastungen berücksichtigt werden, die im Zuge der Bereitstellung der Produktionsmittel während der landwirtschaftlichen Produktion, bei der industriellen Produktion von RME sowie beim Endverbrauch auftreten (UBA, 1993). Maßgeblich sei insbesondere die Bewertung des Treibhauseffektes aufgrund der Emissionen von $\mathrm{CO}_{2}, \mathrm{~N}_{2} \mathrm{O}$ und $\mathrm{CH}_{4}$ während des gesamten "Lebensweges" von RME. Die Klimawirksamkeit der Spurengase wird mit den GWP-Werten (Global Warming Potential) beschrieben, die deren relative Wirksamkeit im Vergleich zu $\mathrm{CO}_{2}$ wiedergeben (IPCC, 1995).

In Ökobilanzen von KALTSCHMITT und REINHARD (1997) und WOLFENSBERGER und DINKEL (1997) wird das bei der energetischen Nutzung von RME freiwerdende $\mathrm{CO}_{2}$ als klimaneutral bewertet, da die äquivalente Menge $\mathrm{CO}_{2}$ über die Photosynthese der Atmosphäre entzogen wird. Unberücksichtigt bleiben in den bisherigen Schätzungen die Auswirkungen des Anbaus land- und forstwirtschaftlicher Nutzpflanzen wie, z.B. WRaps, auf die Stoffflüsse der klimawirksamen Spurengase $\mathrm{N}_{2} \mathrm{O}$ und $\mathrm{CH}_{4}$ in und aus den Böden (UBA, 1993).

Aufgrund der Wirkungskomplexizität der Einflußgrößen sind die Entstehungsbedingungen von $\mathrm{N}_{2} \mathrm{O}$ im Boden in vielen Punkten noch ungeklärt (GRANLI und BOCKMAN, 1994). Bekannt sind eine Reihe von Einflußfaktoren wie Vegetation und Fruchtart, Bodenart, Wassergehalt des Bodens, Bodentemperatur wie auch Art und Menge des ausgebrachten Stickstoffdüngers (BouWMAN, 1990; EichneR, 1990; Sahrawat und KeENEY, 1986). Der Einfluß des Pflanzenaufwuchses auf die $\mathrm{N}_{2} \mathrm{O}$-Emissionen von Böden wird allerdings kontrovers diskutiert. CRIBBS und MILLS (1979) berichten von höheren $\mathrm{N}_{2} \mathrm{O}$-Emissionen auf bewachsenen Flächen, während AULAKH et al. (1982) und DUXBURRY et al. (1982) höhere Emissionen auf Bracheflächen aufzeigen. In der vorliegenden Literatur sind keine Hinweise auf Langzeituntersuchungen über den Einfluß verschiedener Fruchtarten auf die $\mathrm{N}_{2} \mathrm{O}$ - 
Emissionen zu finden. Gleiche Ausführungen sind zu Fragen der $\mathrm{CH}_{4}$-Flüsse zu machen.

Die Höhe der $\mathrm{N}_{2} \mathrm{O}$-Emissionen von Böden wird nach DAvidson (1991) maßgeblich vom Vorhandensein von $\mathrm{NH}_{4}{ }^{+}$und $\mathrm{NO}_{3}^{-}$bestimmt. Einige landwirtschaftliche Nutzpflanzen hinterlassen nach der Ernte auch bei ordnungsgemäßer N-Düngung beachtliche Mengen an mineralischem Stickstoff im Boden. Hierzu zählen die Kartoffel (FISCHER et al., 1992) und der Raps (LICKFETT, 1993). Nach dem Anbau von WRaps kommt es nach LICKFETT (1993) auch bei unterlassener N-Düngung im Herbst und Winter im Gegensatz zum Getreide i.d.R. zu einer Anreicherung von mineralischem $\mathrm{N}$ im Boden. Beträge von 100 und $120 \mathrm{~kg} \mathrm{ha}^{-1} \mathrm{~N}_{\min }$ werden selbst auf mittelschweren und schweren Lehmböden häufig überschritten (Lickfett, 1993). Dieses läßt vermuten, daß es nach dem Anbau von Raps, der als nachwachsender Rohstoff über die Produktion von RME zu einer Entlastung der Umwelt beitragen soll, zu erhöhten $\mathrm{N}_{2} \mathrm{O}$-Emissionen aus den Böden kommt.

Darüber hinaus liegen keine Untersuchungen darüber vor, ob von dieser Fruchtart aufgrund der ihr zugesprochenen bodenlockernden Wirkung sowie aufgrund des Anfalls hoher Mengen an leicht umsetzbaren Ernterückständen besondere Auswirkungen auf die $\mathrm{CH}_{4}$-Flüsse des Bodens ausgehen.

Die Stickstoffmetabolik des Bodens ist aber auch abhängig von Bodenart und Bodentyp. So ist zu erwarten, daß die $\mathrm{N}_{2} \mathrm{O}$-Entstehung und dessen Flüsse durch die chemischen, physikalischen und biologischen Eigenschaften der Bodenhorizonte maßgeblich gesteuert werden. Gley-Auenböden, die in das Forschungsthema der vorliegenden Arbeit einbezogen sind, stellen zwar keinen charakteristischen Standort für den Rapsanbau dar, lassen aber aufgrund unterschiedlicher Eigenschaften ihrer Horizonte eine Mannigfaltigkeit der Prozesse des N-Umsatzes erwarten. Aus Forschungsvorhaben, die dieser Arbeit vorausgingen, liegen bereits nähere Untersuchungen über den Nitratumsatz eines Gley-Auenbodens in einer Rapsfruchtfolge vor (PRZEMECK und LICKFETT, 1992). Ziel der vorliegenden Arbeit ist es, Auswirkungen der Ackerfrüchte einer Rapsfruchtfolge auf die Flüsse von Lachgas und Methan als klimarelevante Spurengase in diesem Gley-Auenboden festzustellen und zu quantifizieren. Dabei soll auch die Bedeutung der N-Düngung berücksichtigt werden. Diesen Fragenkomplex umfaßt der erste Teil der Untersuchungen im Rahmen von Felduntersuchungen. Darüber hinaus ist in dem Forschungsprojekt die Herkunft des $\mathrm{N}_{2} \mathrm{O}$ in dem Bodenprofil zu ergründen sowie ein durch Denitrifikation vermuteter erheblicher $\mathrm{N}$-Verlust experimentell aufzuklären. Hierfür wurden im zweiten Teil der Arbeit Modelluntersuchungen an ungestörten Bodensäulen aus drei Horizonten des Bodens der Versuchsflächen durchgeführt. 
Vor dem Hintergrund einer Raps-Anbaufläche von ca. 914.000 ha (1997) in Deutschland erscheint es schließlich gerechtfertigt, eine ökologische Bewertung des Rapsanbaus aufgrund der vorliegenden Ergebnisse mit Blick auf $\mathrm{N}_{2} \mathrm{O}$ und $\mathrm{CH}_{4}$ abzuschätzen. 


\section{$2 \quad$ Literaturübersicht}

\section{$2.1 \quad \mathrm{~N}_{2}$ O-Bildung in Böden}

\subsection{1 Ökologische Bedeutung}

Distickstoffoxid $\left(\mathrm{N}_{2} \mathrm{O}\right)$ ist ein Treibhausgas, welches in der Stratosphäre den Abbau von Ozon zusätzlich beschleunigt. Seit Beginn der Industrialisierung in der Mitte des 18. Jahrhunderts ist die $\mathrm{N}_{2} \mathrm{O}$-Konzentration in der Atmosphäre um ca. $15 \%$ von 275 ppbv auf 311 ppbv gestiegen (Tab. 2-1). Es wird erwartet, daß die $\mathrm{N}_{2} \mathrm{O}$ Konzentration im Jahr 2030 im Bereich von 350 bis 450 ppbv liegen wird (KRALOVA et al., 1992). Der stetige Anstieg ist zurückzuführen auf die zunehmende anthropogene Emission dieses Spurengases, hervorgerufen durch die Verbrennung fossiler Brennstoffe im Energie- und Verkehrssektor, durch die Rodung von Wäldern sowie durch die Intensivierung der Landwirtschaft (N-Düngung) (USEPA, 1989). Die jährlichen Emissionen in die Atmosphäre stiegen seit der vorindustriellen Zeit um $4,5( \pm 0,6) \mathrm{Tg} \mathrm{N}$ O-N, was einen Anstieg von ca. $50 \%$ bedeutet (GRANLI UND BOCKMAN, 1994).

Tab. 2-1: Charakteristik des Treibhausgases $\mathrm{N}_{2} \mathrm{O}$ in der Atmosphäre (IPCC 1994; IPCC 1995)

\begin{tabular}{l|c}
\hline Konzentration in vorindustrieller Zeit (1750-1800) [ppbv] & 275 \\
Heutige Konzentration [ppbv] & 311 \\
Gegenwärtige Zunahme pro Jahr [\%] & 0,25 \\
Verweildauer in der Atmosphäre (Jahre) & 120 \\
Spezifische Treibhauswirksamkeit bezogen auf die gleiche & 320 \\
Masse $\mathrm{CO}_{2}$ (Zeithorizont: 100 Jahre) & 6 \\
\hline Anteil am anthropogenen Treibhauseffekt [\%] & 6 \\
\hline
\end{tabular}

Die Zunahme der $\mathrm{N}_{2} \mathrm{O}$-Konzentration in der Atmosphäre ist von ökologischer Bedeutung, da sie für mögliche globale Klimaveränderungen mitverantwortlich zu machen ist. $\mathrm{N}_{2} \mathrm{O}$ gilt als "Treibhausgas" der Atmosphäre (SAUERBECK und ISERMANN, 1993). Aufgrund seines hohen spezifischen Treibhauspotentials ist es trotz seiner geringen Konzentration zu ca. $6 \%$ am derzeitigen anthropogenen Treibhauseffekt beteiligt (Tab. 2-1). Der Treibhauseffekt beruht darauf, daß einfallende, kurzwellige Sonnenstrahlung von der Erdoberfläche als langwellige Wärmestrahlen wieder 
abgegeben wird, die durch Treibhausgase absorbiert werden können. Dieses führt zu einer Erwärmung der Erdatmosphäre. Die natürlich vorkommenden Treibhausgase heben nach DUXBURY et al. (1982) die mittlere Temperatur an der Erdoberfläche von $-18^{\circ} \mathrm{C}$ auf $15^{\circ} \mathrm{C}$ an.

Eine zusätzliche, anthropogen verursachte Erhöhung der natürlichen Treibhausgaskonzentration führt $\mathrm{zu}$ einer zusätzlichen Erwärmung der Erdatmosphäre und somit zum "anthropogenen Treibhauseffekt". Die hohe chemische Stabilität von $\mathrm{N}_{2} \mathrm{O}$ in der Troposphäre, die seine hohe Verweildauer in der Atmosphäre (120 Jahre; Tab. 2-1) erklärt, führt dazu, daß $\mathrm{N}_{2} \mathrm{O}$ durch Diffusion in die Stratosphäre gelangen kann und dort zusätzlich zum Abbau der Ozonschicht beiträgt (CRUTZEN et al., 1979). In der Stratosphäre reagiert es mit photochemisch erzeugtem atomaren Sauerstoff und bildet dabei $\mathrm{NO}$, das mit Ozon unter Bildung von $\mathrm{NO}_{2}$ und $\mathrm{O}_{2}$ reagiert. Die primär durch $\mathrm{N}_{2} \mathrm{O}$ verursachte Abnahme der Ozonschicht soll bei ungefähr $5 \%$ liegen (UBA, 1992). Eine zunehmende Zerstörung der stratosphärischen Ozonschicht fördert den Anstieg der UV-B-Strahlung auf der Erdoberfläche. CRUZEN und ENHALT (1977) prognostizieren, daß bei einer Verdoppelung der $\mathrm{N}_{2} \mathrm{O}$-Konzentration in der Atmosphäre die UV-B-Strahlung an der Erdoberfläche um ca. $20 \%$ steigen wird.

\subsubsection{Prozesse der $\mathrm{N}_{2} \mathrm{O}$-Bildung in Böden}

Als Quelle für Distickstoffmonoxid in Böden gelten die Nitrifikation und die Denitrifikation (FIRESTONE et al., 1979; BREMNER UND BLACKMER, 1981; SAHRAWAT Und KeEney, 1996; Robertson, 1989; Bouwman, 1990; Smith und Arah, 1990; SHARP, 1991; GISI et al., 1990; BADR und Probert 1992; BATJES, 1992). An beiden Prozessen der $\mathrm{N}_{2} \mathrm{O}$-Bildung können sowohl autotrophe (lithotrophe) als auch heterotrophe (organotrophe) Mikroorganismen beteiligt sein (BLACKMER et al., 1980; BREMNER und BLACKMER, 1981; ANDERSON Und LEVINE,1986; BLACKMER und CERRATO, 1986; Tortoso und HutChINSON, 1990; SKIBA et al., 1993; PenNington und Ellis, 1993). Für terrestrische Böden wird in der Literatur die $\mathrm{N}_{2} \mathrm{O}$-Produktion in Verbindung mit der Denitrifikation bedeutender angesehen als mit der Nitrifikation (FIREstone et al., 1980; SeILER und ConRAD, 1981; RUdAZ et al., 1991; Granli und BOCKMAN, 1994). Jedoch dürfen die Emissionen aus der Nitrifikation nicht unterschätzt werden, da besonders in ariden Gebieten wasserungesättigte Verhältnisse in Ackerböden und damit eine die Nitrifikation fördernde $\mathrm{O}_{2}$-Versorgung in Böden viel häufiger auftreten als wassergesättigte Bedingungen (FIRESTONE und DAVIDSON, 1989; RUdAZ et al., 1991; KRALOVA et al., 1992; MuMmeY et al., 1994).

In Abbildung 2-1 ist die Bildung und Emission von $\mathrm{N}_{2} \mathrm{O}$ durch die genannten Prozesse der mikrobiellen N-Transformation anhand des "hole-in-the-pipe" Modells 
nach DAVIDSON (1991) dargestellt. Es ist zu erkennen, daß im Bereich der wassergefüllten Porenräume des Bodens die Höhe der $\mathrm{N}_{2} \mathrm{O}$-Freisetzung von den Prozeßraten der Nitrifikation und der Denitrifikation abhängt. Die Höhe der N-Flüsse durch die Prozeßröhren wird dabei maßgeblich vom Vorhandensein der Quellsubstrate $\mathrm{NH}_{4}{ }^{+}$und $\mathrm{NO}_{3}{ }^{-}$bestimmt. Die "Leckgröße" in den Prozeßröhren bestimmt den relativen Stickstoffanteil, der während dieser Prozesse als $\mathrm{N}_{2} \mathrm{O}$ freigesetzt wird. Die aeroben Nitrifikanten können $\mathrm{N}_{2} \mathrm{O}$ nur bilden, nicht aber verbrauchen. Die unter Sauerstoffmangel denitrifizierenden Bakterien hingegen können $\mathrm{N}_{2} \mathrm{O}$ sowohl produzieren (Reduktion von Nitrit zu $\mathrm{N}_{2} \mathrm{O}$ ) als auch verbrauchen (Reduktion von $\mathrm{N}_{2} \mathrm{O}$ zu N $\mathrm{N}_{2}$ ). Beim Transport vom Produktionsort durch den Boden in die Atmosphäre kann $\mathrm{N}_{2} \mathrm{O}$ je nach Diffusionsbedingungen jederzeit weiter zu $\mathrm{N}_{2}$ reduziert werden. Die Diffusionsbedingungen des Bodens wirken hierbei nicht nur auf die Transportrate von $\mathrm{N}_{2} \mathrm{O}$, sondern beeinflussen auch seine Produktions- und Reduktionsrate. Das Schema zeigt, daß die Emission von $\mathrm{N}_{2} \mathrm{O}$ nicht gleichzusetzen ist mit der Produktion von $\mathrm{N}_{2} \mathrm{O}$ im Boden.

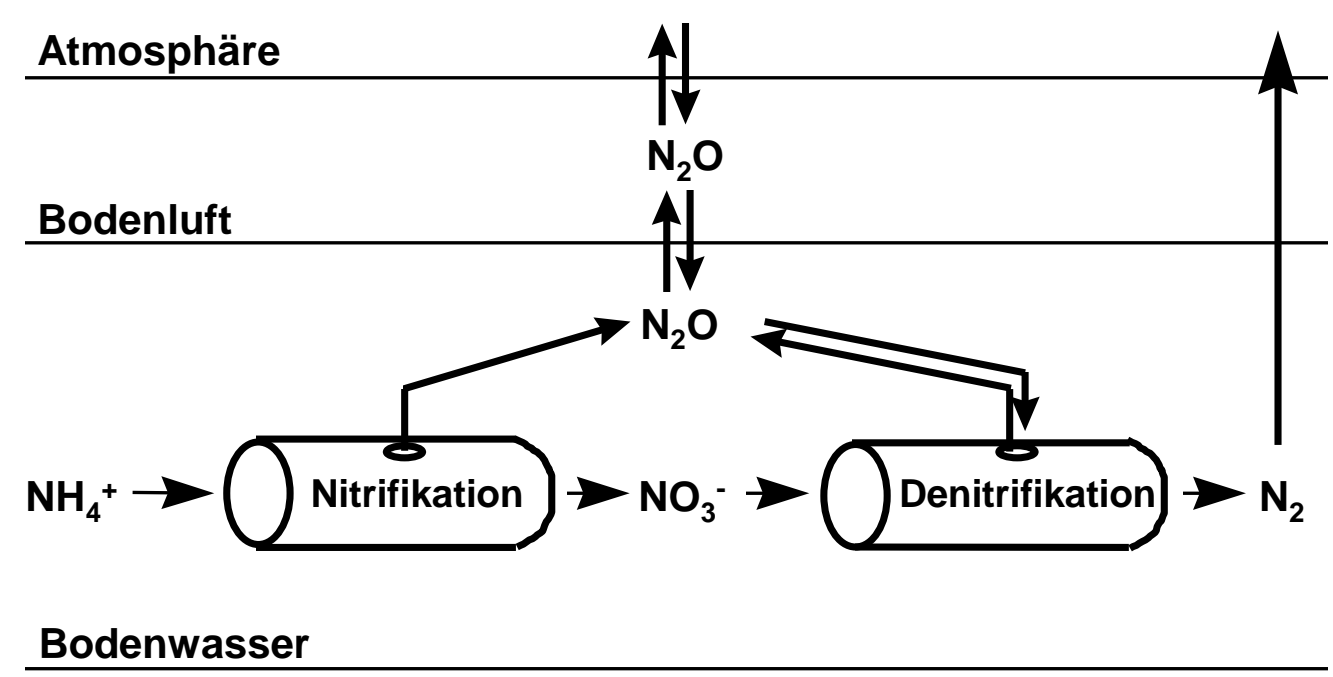

Abb. 2-1: $\quad$ Bildung und Emission von $\mathrm{N}_{2} \mathrm{O}$ durch Nitrifikation und Denitrifikation (nach DAVIDSON, 1991)

Die wichtigsten Steuergrößen der $\mathrm{N}_{2} \mathrm{O}$-bildenden Prozesse sind in Abb. 2-2 in Anlehnung an ROBERTSON (1989) dargestellt, die ausführlich von FIRESTONE und Davidson (1989), Robertson (1989), BATJes Und BRIDGES (1992), BeEse (1993), BOUWMAN (1994) und GRANLI und BOCKMAN (1994) behandelt wurden. 


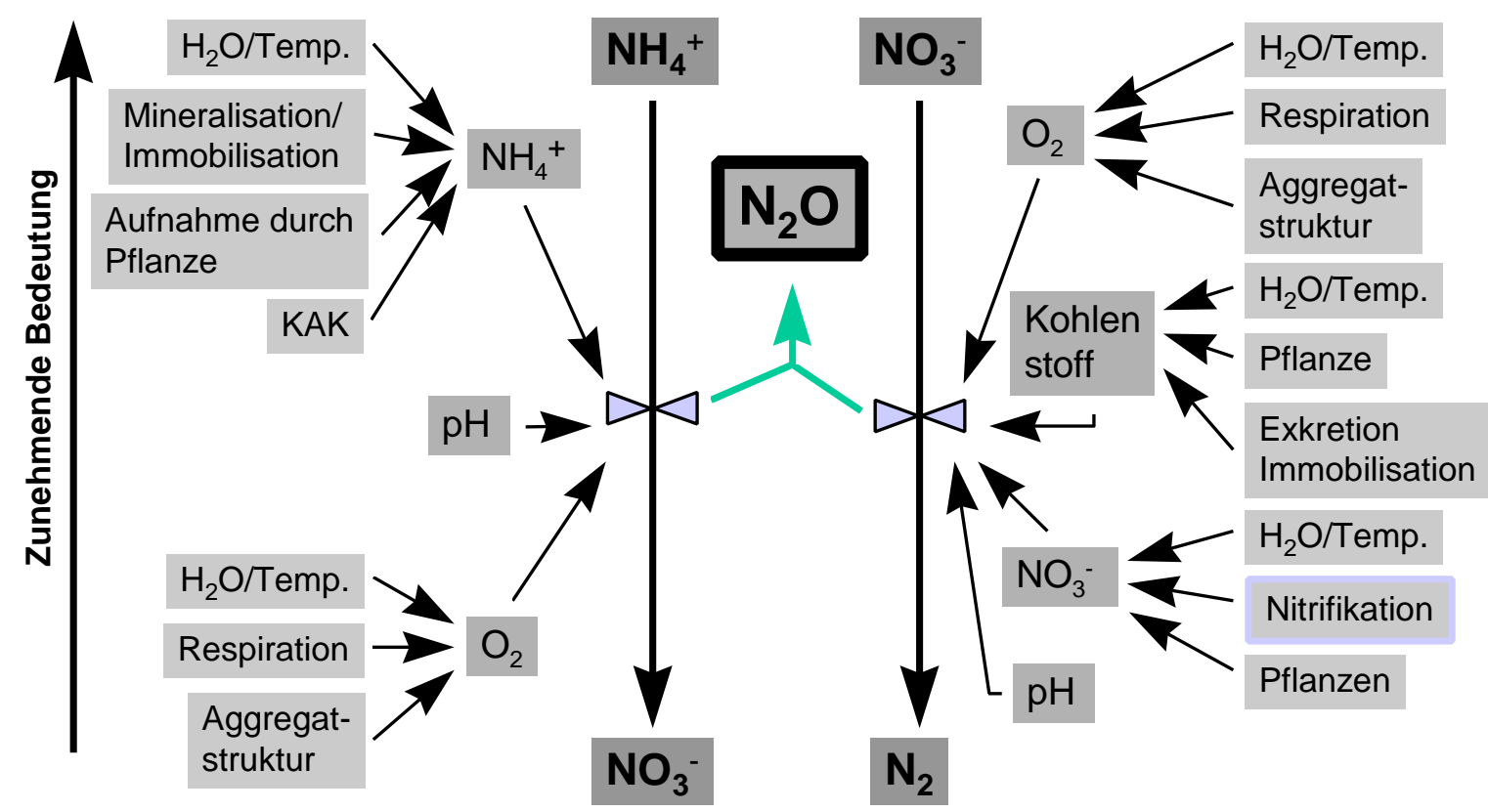

Nitrifikation

Denitrifikation

Abb. 2-2: $\quad$ Einflußfaktoren der $\mathrm{N}_{2} \mathrm{O}-, \mathrm{NO}$ und $\mathrm{N}_{2}$-bildenden Prozesse (verändert nach ROBERTSON, 1989)

\section{Nitrifikation}

Die Nitrifikation ist die mikrobielle Oxidation von Ammonium $\left(\mathrm{NH}_{4}^{+}\right)$über Nitrit $\left(\mathrm{NO}_{2}{ }^{-}\right)$ zu Nitrat $\left(\mathrm{NO}_{3}{ }^{-}\right)$(Abb. 2-3). Die Nitrifizierer nutzen hierbei die durch die Bildung von Nitrit $(272 \mathrm{~kJ})$ und Nitrat $(79 \mathrm{~kJ})$ freigesetzte Energie zur Lebenserhaltung. In landwirtschaftlich genutzten Böden dominiert die Ammoniumoxidation durch chemoautotrophe Nitrifizierer. Der erste Reaktionsschritt, die Oxidation von Ammonium zu Nitrit, wird hierbei von obligat aeroben Bakterien der Gattung Nitrosomonas, Nitrosococcus, Nitrospira und Nitrosolobus vollzogen (BLACKMER et al., 1980, BREMNER und BLACKMER, 1981). Die zweite Reaktion, die Oxidation von Nitrit zu Nitrat, erfolgt durch die Bakterien der Gattung Nitrobacter (LIPSCHULTZ et al., 1981). Diese Reaktion läuft schneller ab als die erste, deshalb findet sich Nitrit im Boden nur in Spuren.

Die Nitrifikation wird durch zahlreiche Einflußfaktoren wie z.B. die Konzentration an Ammonium in der Bodenlösung, den Sauerstoffpartialdruck und den pH-Wert des Bodens beeinflußt. Diese Einflußgrößen werden wiederum durch andere Faktoren mitbestimmt (Abb. 2-2). Der relative Anteil der $\mathrm{N}_{2} \mathrm{O}$-Produktion bei der Nitrifikation kann durch das $\mathrm{N}_{2} \mathrm{O} / \mathrm{NO}_{3}{ }^{-}$-Verhältnis beschrieben werden. 
FIRESTONE und DAVIDSON (1989) zeigten, daß dieses Verhältnis durch die Sauerstoffverfügbarkeit und den pH-Wert des Bodens beeinflußt wird. Obwohl die Autoren bei abnehmendem Sauerstoffpartialdruck und sinkendem $\mathrm{pH}$-Wert meistens einen Anstieg des $\mathrm{N}_{2} \mathrm{O} / \mathrm{NO}_{3}{ }^{-}$-Verhältnisses beobachten, konnten sie direkte Rückschlüsse auf die absolute Höhe der $\mathrm{N}_{2} \mathrm{O}$-Produktion hieraus jedoch nicht ableiten, da diese Einflußfaktoren gleichzeitig die Nitrifikationsraten selbst verringerten.

Der biochemische Prozeß der $\mathrm{N}_{2} \mathrm{O}$-Bildung während der Nitrifikation ist noch nicht vollständig aufgeklärt. PAPEN und RENNENBERG (1990) beschreiben sowohl die mikrobielle Oxidation von Hydroxylamin $\left(\mathrm{NH}_{2} \mathrm{OH}\right)$ als auch die chemische Umwandlung von Nitroxyl (HNO) zu $\mathrm{N}_{2} \mathrm{O}$ als mögliche Ursache. Andere Autoren gehen davon aus, daß $\mathrm{N}_{2} \mathrm{O}$ bei Sauerstoffmangel direkt aus Nitrit gebildet wird (Seiler und ConRad, 1981; Firestone und Davidson, 1989). Diese "NitrifiziererDenitrifikation" gewinnt bei abnehmender Sauerstoffverfügbarkeit des Bodens an Bedeutung (GrofFMAN, 1991). Es handelt sich dabei um einen reduktiven Prozeß, bei dem unter Sauerstoffmangel einige Bakterienarten das Nitrit, welches nicht zu Nitrat oxidiert werden kann, als Elektronenakzeptor nutzen. Mit diesem Reduktionsprozeß wird gleichzeitig erreicht, daß das Zellgift Nitrit nicht im Boden akkumuliert wird (FIRESTONE und DAVIDSON, 1989).

In der Regel soll die $\mathrm{N}_{2} \mathrm{O}-\mathrm{N}$-Freisetzung deutlich weniger als $1 \%$ der nitrifizierten Stickstoffmenge betragen (FIRESTONE und DAVIDSON, 1989). FLESSA et al. (1996) fanden auf einem Lößlehm nach Ammoniumdüngung eine $\mathrm{N}_{2} \mathrm{O}$-Freisetzung von lediglich $0,06 \%$ des nitrifizierten Stickstoffs.

\section{Denitrifikation}

Denitrifikation ist die dissimilatorische Reduktion von Nitrat $\left(\mathrm{NO}_{3}{ }^{-}\right)$oder Nitrit $\left(\mathrm{NO}_{2}{ }^{-}\right)$ zu Stickstoffmonoxid (NO), Distickstoffmonoxid $\left(\mathrm{N}_{2} \mathrm{O}\right)$ oder molekularem Stickstoff $\left(\mathrm{N}_{2}\right)$ (Abb. 2-3). Die Denitrifikanten gewinnen dabei ihre Energie aus der Oxidation eines Reduktionsmittels: Die heterotrophen Denitrifikanten aus der Oxidation von organischen Kohlenstoffverbindungen (SCHLEGEL, 1985), die autotrophen Denitrifikanten aus reduzierten Schwefelverbindungen (KöLLE et al., 1985; BÖTTCHER, 1992) wie auch aus $\mathrm{Fe}^{2+}$ (BURESH und MORAGHAN, 1976; LIND und Pedersen, 1976). 


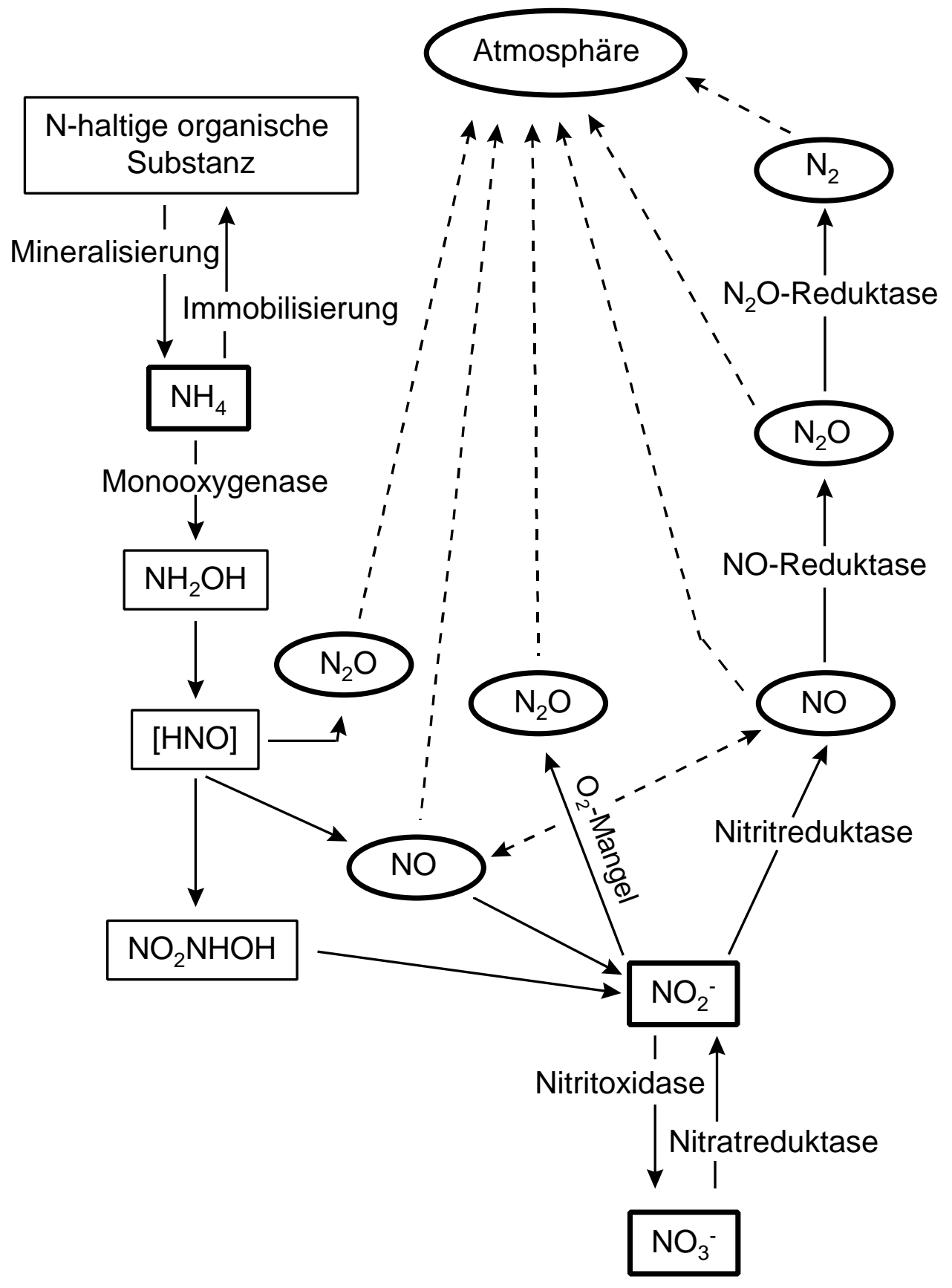

Abb. 2-3: $\quad$ Stickstoffumsetzung durch die autotrophe Nitrifikation und durch die Denitrifikation. Die gestrichelten Linien kennzeichnen ein mögliches Entweichen gasförmiger NVerbindungen in die Atmosphäre (verändert nach FIRESTONE und DAVIDSON, 1989). 
Der Kohlenstoff dient heterotrophen Organismen nicht nur als Elektronendonator, sondern ist auch für den assimilatorischen Stoffwechsel notwendig. Zur Beschreibung der organotrophen Denitrifikation unter sauren (Gl. 1) und alkalischen Bedingungen (Gl. 2) geben BURFORD und BREMNER (1975) und TRUDELL et al. (1996) folgende stöchiometrische Gleichungen an:

$$
\begin{aligned}
& 5 \mathrm{CH}_{2} \mathrm{O}+4 \mathrm{NO}_{3}{ }^{-}+4 \mathrm{H}^{+} \Rightarrow 2 \mathrm{~N}_{2}+5 \mathrm{CO}_{2}+7 \mathrm{H}_{2} \mathrm{O} \\
& 5 \mathrm{CH}_{2} \mathrm{O}+4 \mathrm{NO}_{3}{ }^{-} \Rightarrow 2 \mathrm{~N}_{2}+5 \mathrm{HCO}_{3}{ }^{-}+\mathrm{H}^{+}+2 \mathrm{H}_{2} \mathrm{O}
\end{aligned}
$$

Bei der autotrophen Denitrifikation dienen den Mikroorganismen primäre Schwefelverbindungen, wie z.B. Pyrit und Melnikovit oder amorphe sulfidische Sekundärverbindungen als Energiequelle (BÖTTCHER, 1992). Die Reaktion verläuft nach KöLLE et al. (1985) gemäß folgender Gleichung:

$$
5 \mathrm{FeS}_{2}+14 \mathrm{NO}_{3}{ }^{-}+4 \mathrm{H}^{+} \Rightarrow 7 \mathrm{~N}_{2}+10 \mathrm{SO}_{4}{ }^{2+}+5 \mathrm{Fe}^{2+}+2 \mathrm{H}_{2} \mathrm{O}
$$

Die autotrophe Denitrifikation wurde in verschiedenen Grundwassereinzugs-gebieten beobachtet (Kölle et al., 1983; VAN BeEK et al., 1988; Postma et al., 1991). In Untersuchungen von FUNK (1993), der den Zusammenhang zwischen Sulfatbildung und Denitrifikation in Lößprofilen beschreibt, kann die Sulfatproduktion nur einen Teil der beobachteten Nitratverluste erklären. Daraus schließt er, daß auch das bei der Eisensulfidreduktion gebildete freie $\mathrm{Fe}^{2+}$ als Reduktionsmittel berücksichtigt werden muß:

$$
5 \mathrm{Fe}^{2+}+\mathrm{NO}_{3}{ }^{-}+7 \mathrm{H}_{2} \mathrm{O} \Rightarrow 5 \mathrm{FeOOH}+0,5 \mathrm{~N}_{2}+9 \mathrm{H}^{+}
$$

Denitrifikanten sind fakultativ anaerob; sie sind in der Lage, anstatt des Sauerstoffs auch sauerstoffhaltige Stickstoffverbindungen als Elektronenakzeptoren zu nutzen. Typische Vertreter von Denitrifikanten sind Bakterien der Gattung Pseudomonas, Thiobacillus denitrificans, Corynebacterium, Paracoccus, Chromobacterium, Hyphomicrobium und Serratina (INGRAHAM, 1981; PAYNE, 1995). Die meisten denitrifizierenden Bakterien sind nur in der Lage, einen oder zwei Reaktionsschritte der gesamten Denitrifikation auszuführen (GERMON, 1985). MALINOWSKY und OTTOW (1995) weisen darauf hin, daß auch einige Pilzarten (u.a. Fusarium, Acremonium und Aspergillus) Nitrat reduzieren können. Nitratreduktion durch reduzierte SVerbindungen wird bei Thiobacillus denitrificans (KöLLE et al., 1983) und durch $\mathrm{Fe}^{2+}$ durch Gallionella ferruginea (POSTMA, 1990) beobachtet.

Die Denitrifikation als anaerobem Prozeß ist in terrestrischen Böden von der $\mathrm{O}_{2}$ Konzentration abhängig (Abb. 2-2). Der Sauerstoffpartialdruck des Bodens wird durch die Sauerstoffnachlieferung aus der Atmosphäre und durch den respiratorischen $\mathrm{O}_{2}$-Verbrauch gesteuert. Die Denitrifikation kann daher sowohl durch eine Verringerung der $\mathrm{O}_{2}$-Diffusion in den Boden durch erhöhte Bodenwassergehalte als auch durch einen gesteigerten mikrobiellen 
Sauerstoffverbrauch im Zuge der Zersetzung leicht verfügbarer organischer Substanz ausgelöst werden (FLESSA und BEESE, 1995). Als weitere Einflußfaktoren werden hinsichtlich der Respirationsleistung die Bodentemperatur und hinsichtlich der $\mathrm{O}_{2}$-Diffusion die Aggregatstruktur (Bodenverdichtungen) genannt (Abb. 2-2, ROBERTSON, 1989). Der für die Mikroorganismen verfügbare Kohlenstoff selbst stellt einen limitierenden Faktor für die Denitrifikation dar (WEIER et al., 1993 b,c). Jedoch kann auch bei geringerer Kohlenstoffverfügbarkeit in der Zone unterhalb des Wurzelraumes Nitrat reduziert werden. Hier sind die Verweilzeiten des Nitrates in der Regel wesentlich höher als im Oberboden, so daß für eine Reduktion mehr Zeit bleibt. Außerdem ist die Wahrscheinlichkeit der Bildung anaerober Kompartimente aufgrund der $\mathrm{O}_{2}$-Zehrung im Oberboden und langer $\mathrm{O}_{2}$-Diffusionswege durch größere Entfernungen von der Bodenoberfläche erhöht, zudem können autotrophe Reduktionsprozesse auftreten (ROLLAND, 1995).

Der Reduktionsschritt vom Nitrit zum Distickstoffoxid als Intermediärprodukt der Denitrifikationskette ist noch nicht vollständig geklärt. Da nicht alle Denitrifizierer Nitrat vollständig zum $\mathrm{N}_{2}$ reduzieren, tritt $\mathrm{N}_{2} \mathrm{O}$ auch als Produkt der Denitrifikation auf. Der relative $\mathrm{N}_{2} \mathrm{O}$-Anteil an den gasförmigen $\mathrm{N}$-Verlusten des Bodens wird von vielen Steuergrößen beeinflußt. Das Verhältnis von $\mathrm{N}_{2} \mathrm{O} / \mathrm{N}_{2}$ sinkt mit zunehmender Feuchtigkeit des Bodens (ERIKSEN und HolTEM-HARTWIG, 1993; BURESH et al., 1993); Böden mit einem hohen Wassergehalt geben daher weniger $\mathrm{N}_{2} \mathrm{O}$ an die Atmosphäre ab als entwässerte Böden. Im Gegensatz dazu wurden beträchtliche $\mathrm{N}_{2} \mathrm{O}$ Emissionen aus bewässerten Reisfeldern gemessen (BouwmAN et al., 1990). Dieses zeigt, daß verschiedene denitrifizierende Bakterien unterschiedlich auf Sauerstoffund Wassergehalt des Bodens reagieren (MUNCH und OTTOW, 1987). Die denitrifikative Bildung von $\mathrm{N}_{2} \mathrm{O}$ in aeroben Böden kann durch punktuelle anaerobe Zonen ("hot spots") möglich sein (HEINEMEYER et al., 1988, FLESSA und BEESE, 1995). Bei diesen "hot spots" kann es sich zum Beispiel um eine Anhäufung von eingearbeiteten, leicht mineralisierbaren Ernteresten handeln. Durch die Mineralisierung dieser Erntereste mit nachfolgender Nitrifikation im aeroben Bereich um diese "hot spots" herum wird zusätzlich ausreichend Nitrat für eine Denitrifikation im anaeroben Bereich zur Verfügung gestellt. Durch eine hohe Mineralisationsrate in den aeroben Zonen nimmt der Gehalt an Elektronenakzeptoren stark zu, wodurch die Denitrifikationsrate in einem größeren Maße bestimmt wird als unter total anaeroben Bedingungen (ABOU-SEADA und OTTOW, 1985).

Wenn eine im Vergleich zum Nitratangebot mangelhafte Kohlenstoffverfügbarkeit auftritt, werden die N-Oxide von den Denitrifikanten nur unvollständig genutzt. Stickstoffmonoxid wie auch Distickstoffoxid treten dann als Endprodukt auf und entweichen in die Atmosphäre (Bouwman et al., 1990). WEIER et al. (1993 a) fanden, 
daß das $\mathrm{N}_{2} \mathrm{O} / \mathrm{N}_{2}$-Verhältnis unter denitrifizierenden Bedingungen mit abnehmendem Gehalt an verfügbarem Kohlenstoff stieg.

Weitere Faktoren, die das $\mathrm{N}_{2} \mathrm{O} / \mathrm{N}_{2}$-Verhältnis beeinflussen, sind der $\mathrm{pH}$-Wert und die Temperatur (Abb. 2-2). Die Aktivität der $\mathrm{N}_{2} \mathrm{O}$-Reduktase wird bei Temperaturen $<5^{\circ} \mathrm{C}$ und $\mathrm{pH}-$ Werten $<5$ überproportional verringert, so daß das $\mathrm{N}_{2} \mathrm{O} / \mathrm{N}_{2}$-Verhältnis in sauren Böden und bei Temperaturen um den Gefrierpunkt deutlich ansteigt (Weier und Gilliam, 1986; Melin und NOMmiK, 1983).

Der relative Anteil der $\mathrm{N}_{2} \mathrm{O}-\mathrm{N}-$ Freisetzung an der Gesamt-N-Freisetzung der Denitrifikation kann zwischen 0 und $100 \%$ betragen und ist damit nur unzureichend abschätzbar (GRANLI und BOCKMAN, 1994).

\subsection{3 $\quad \mathrm{N}_{2} \mathrm{O}$-Emissionen aus landwirtschaftlich genutzten Flächen}

Eine Abschätzung der $\mathrm{N}_{2} \mathrm{O}$-Emissionen aus landwirtschaftlich genutzten Flächen ist mit großen Unsicherheiten behaftet. In der vorliegenden Literatur liegen keine speziellen Langzeituntersuchungen über den Einfluß von Winterraps auf die Lachgasemissionen vor. Die $\mathrm{N}_{2} \mathrm{O}$-Emissionsraten verschiedener Standorte unterliegen starken Schwankungen. Dieses gilt im besonderen Maße für Agrarökosysteme (BouWMAN, 1990). Aus den bisher erhobenen Daten geht jedoch deutlich hervor, daß die $\mathrm{N}_{2} \mathrm{O}$-Emissionen aus landwirtschaftlich genutzten Flächen deutlich über denen von unkultivierten Flächen bzw. naturnahen Ökosystemen liegen. Grund für die besonders starken Schwankungen der $\mathrm{N}_{2} \mathrm{O}$-Emissionen landwirtschaftlich genutzter Flächen wie auch für das höhere Emissionsniveau aus Agrarökosystemen könnte sein, daß die bodenseitigen Einflußfaktoren der $\mathrm{N}_{2} \mathrm{O}$ Freisetzung maßgeblich durch Bewirtschaftungsmaßnahmen beeinflußt werden (vgl. GRANLI UND BOCKMANN, 1994). Zu den Einflußfaktoren des Bodens gehören u.a. der Bodenwassergehalt, der Gehalt an organischem Kohlenstoff und Stickstoff, die Textur, die Porosität und der pH-Wert.

Bewirtschaftungsmaßnahmen, die die $\mathrm{N}_{2} \mathrm{O}$-Emission erhöhen, können die Bodenbearbeitung selbst (STALEY et al., 1990; HANSEN et al., 1993; RuSER et al., 1997), Be- und Entwässerung (MosIER et al., 1986) sowie auch das Management der Ernterückstände (FLESSA und BEESE, 1995) sein. Diese Maßnahmen haben i.d.R. eine Steigerung des N-Umsatzes im Boden zur Folge. Die deutlichsten Auswirkungen zeigt jedoch die Stickstoffdüngung. Starke $\mathrm{N}_{2} \mathrm{O}$-Emissionsereignisse, hervorgerufen durch den Überschuß an Ausgangssubstrat für die Nitrifikation $\left(\mathrm{NH}_{4}{ }^{+}\right)$ wie auch für die Denitrifikation $\left(\mathrm{NO}_{3}{ }^{-}\right)$, wurden direkt nach den Düngergaben beobachtet. Mit steigender N-Applikationsmenge wurde zumeist auch eine Zunahme der $\mathrm{N}_{2} \mathrm{O}$-Emission beobachtet (BOUWMAN, 1990; VAN KESSEL et al., 1993). Einige 
Arbeiten registrieren auch einen unterschiedlichen Einfluß von $\mathrm{NO}_{3}{ }^{-}$und $\mathrm{NH}_{4}{ }^{+}-$ Dünger auf die $\mathrm{N}_{2} \mathrm{O}$-Emissionen (EICHNER, 1990).

Die $\mathrm{N}_{2} \mathrm{O}$-Emissionen aus hoch mit Stickstoff versorgten, landwirtschaftlich genutzten Böden sind sowohl nach SAUERBECK und ISERMANN (1993) als auch nach GRANLI und BOCKMAN (1994) wesentlich an den weltweit anthropogenen $\mathrm{N}_{2} \mathrm{O}$-Emissionen beteiligt. Als wesentliche Ursache für den Anstieg der globalen atmosphärischen $\mathrm{N}_{2} \mathrm{O}-$ Konzentration gilt der zunehmende Einsatz von mineralischem Stickstoff in der Landwirtschaft, der sich weltweit auf ca. $80 \mathrm{Tg} \mathrm{N} \mathrm{a}^{-1}$ Handelsdünger und etwa die gleiche Menge an Wirtschaftsdünger in den letzten 30 Jahren vervierfacht hat. Hinzu kommt ein schlecht quantifizierbarer Eintrag über die symbiontische N-Fixierung beim Anbau von Leguminosen, der möglicherweise jedoch in einer ähnlichen Größenordnung liegt (DuXBURY et al., 1993; BOUWMAN, 1994).

Da sowohl die Höhe der applizierten N-Menge als auch die applizierte N-Form von Düngerstickstoff hinsichtlich der Höhe der $\mathrm{N}_{2} \mathrm{O}$-Emissionen eine zentrale Rolle spielen, versuchte EICHNER (1990) anhand von Literaturdaten Emissionsfaktoren abzuleiten. Häufig wurden in der Literatur nur Kurzzeiteffekte beschrieben, die nur unter Vorbehalt zur Abschätzung annueller $\mathrm{N}_{2} \mathrm{O}$-Emissionen herangezogen werden konnten. Conrad und SeILer (1980), SAuerbeck und ISERMAnN (1993) wie auch RUZ-JEREZ et al. (1994) berichteten von $\mathrm{N}_{2} \mathrm{O}-\mathrm{N}$ Verlusten, die sich zwischen 0,01 und $3,45 \%$ des eingesetzten Düngerstickstoffs bewegen.

BouWMAN (1994) stellte auf der Grundlage ganzjähriger Untersuchungen unabhängig von der Düngerform einen Zusammenhang zwischen der applizierten Düngermenge und der annuellen $\mathrm{N}_{2} \mathrm{O}-$ Emission dar:

$\mathrm{N}_{2} \mathrm{O}-\mathrm{N}$ Emission $\left[\mathrm{kg}\right.$ ha $\left.\mathrm{a}^{-1}\right]=0,0125 \times \mathrm{N}$-Düngung $\left[\mathrm{kg} \mathrm{ha} \mathrm{a}^{-1}\right]$

Im Mittel wurden 1,25\% des applizierten Stickstoffs als $\mathrm{N}_{2} \mathrm{O}-\mathrm{N}$ emittiert. Die mittlere Basisemission der ungedüngten Referenzflächen betrug $1 \mathrm{~kg} \mathrm{~N}_{2} \mathrm{O}-\mathrm{N}$ ha $\mathrm{a}^{-1}$. Diese Beziehung beruhte auf 20 Datensätzen von 7 Standorten, auf denen überwiegend Mais angebaut wurde. Die dort dargestellte Regression ist deshalb laut BouWMAN (1994) nicht dazu geeignet, die Emissionen anderer Standorte oder anderer Feldfrüchte abzuschätzen. Aufgrund der mangelnden Datenbasis stellt diese Regression trotz großer Unsicherheiten derzeit eine der wichtigsten Grundlagen für die weltweite Abschätzung der $\mathrm{N}_{2} \mathrm{O}$-Emissionen aus landwirtschaftlich genutzten Böden dar (IPCC, 1996).

Um eine düngungsabhängige $\mathrm{N}_{2} \mathrm{O}$-Gesamtemission landwirtschaftlich genutzter Böden abzuschätzen, müssen neben den direkten $\mathrm{N}_{2} \mathrm{O}$-Emissionen auch noch indirekte $\mathrm{N}_{2} \mathrm{O}$-Emissionen des Standortes berücksichtigt werden. Über die Auswaschung von Stickstoff, Bodenerosion oder gasförmige Ammoniakverluste 
kommt es zu einer Stickstoffbelastung angrenzender naturnaher terrestrischer und aquatischer Ökosysteme, die dann ebenfalls erhöhte $\mathrm{N}_{2} \mathrm{O}$-Emissionen aufweisen können. Diese indirekten $\mathrm{N}_{2} \mathrm{O}$-Emissionen tragen wesentlich zur düngungsabhängigen Gesamtemission einer landwirtschaftlich genutzten Fläche bei (DUXBURY et al., 1993) und müssen somit bei deren Abschätzung mit berücksichtigt werden. Die Höhe der indirekten Emissionen ist jedoch bisher weitgehend unbekannt, wird aber meistens auf ca. 0,75\% des gedüngten Stickstoffs (IPCC, 1996) geschätzt. Die Gesamtemissionen als Summe aus direkten und indirekten $\mathrm{N}_{2} \mathrm{O}$-Emissionen betragen laut IPCC (1996) im Mittel 2 \% der Stickstoffdüngung.

\section{2. $\mathrm{CH}_{4}$-Bildung und -Verbrauch in Böden}

\subsection{1 Ökologische Bedeutung}

Methan $\left(\mathrm{CH}_{4}\right)$ ist neben dem Kohlendioxid das wichtigste Treibhausgas. Sein Anteil am anthropogenen Treibhauseffekt wird auf $19 \%$ geschätzt (Tab. 2-2). Seit der Industrialisierung zu Beginn des 19. Jahrhunderts ist die Methankonzentration in der Erdatmosphäre um $145 \%$ auf 1714 ppbv angestiegen, in den letzten 50 Jahren betrug der Konzentrationsanstieg nahezu 1,0 \% im Jahr (BLAKE und ROWLAND, 1988; KHALIL und RASMUSSEN 1990). THOMPSON und CICERONE (1986) vermuten, daß die

Tab. 2-2: $\quad$ Charakteristik des Treibhausgases $\mathrm{CH}_{4}$ in der Atmosphäre (IPCC 1994; IPCC 1995)

\begin{tabular}{l|c}
\hline Konzentration in vorindustrieller Zeit (1750-1800) [ppbv] & 700 \\
Heutige Konzentration [ppbv] & 1714 \\
Gegenwärtige Zunahme pro Jahr [\%] & 0,8 \\
Verweildauer in der Atmosphäre (Jahre) & $12-17$ \\
Spezifische Treibhauswirksamkeit bezogen auf die gleiche & 24,5 \\
Masse $\mathrm{CO}_{2}$ (Zeithorizont: 100 Jahre) & $\mathbf{1 9}$ \\
\hline Anteil am anthropogenen Treibhauseffekt [\%] & \\
\hline
\end{tabular}


atmosphärische Methankonzentration bis zum Jahr 2035 auf 2900 ppbv steigen wird. Dieser Anstieg wird sowohl auf erhöhte Emissionen als auch auf eine Reduzierung der Senkenstärke zurückgeführt. Anthropogene Ursachen machen hierbei ca. 60$80 \%$ der Gesamtemissionen von $520 \pm 80 \mathrm{Tg} \mathrm{CH}_{4} \mathrm{a}^{-1}$ aus. Massentierhaltung, anaerobe Fermentation im Reisanbau oder auf Mülldeponien, Verbrennung von Biomasse sowie die Förderung von fossilen Brennstoffen sind die wichtigsten Prozesse, in denen dieser hohe Anteil an $\mathrm{CH}_{4}$ freigesetzt wurde (WATSON et al., 1992; HEYER, 1993). Aus natürlichen Feuchtgebieten werden ca. $20 \%$ der Gesamtemissionen emittiert (IPCC, 1995). Das den anaeroben Zonen des Bodens entweichende Methan kann zu annähernd $90 \%$ von methanotrophen Bakterien oxidiert werden, wenn es in aerobe Bereiche des Bodens gelangt (CONRAD und RotHFUSS 1991). Die aeroben Bereiche des Bodens wirken als biologische Senke. Das dem Boden entweichende Methan wird in der Atmosphäre zu ca. $90 \%$ durch photochemische Reaktion mit Hydroxyradikalen abgebaut (WATSON et al., 1992). Ein Teil des Methans diffundiert von der Atmosphäre in die Stratosphäre, in der es jedoch nur in geringem Umfang am Ozonabbau beteiligt ist (CRUTZEN, 1991). Die einzige biologische Senke für atmosphärisches Methan stellt die Oxidation in aeroben Böden terrestrischer Ökosysteme dar, wodurch nach neueren Schätzungen etwa 15-60 Tg CH $\mathrm{Ca}^{-1}$ abgebaut werden (CICERONE und OREMLAND, 1988; ScHULZ et al., 1990 und WATSON et al., 1992). Der Beitrag der Böden ist im Vergleich zum Methanabbau in der Atmosphäre zwar gering, jedoch liegt er in der gleichen Größenordnung wie der jährliche $\mathrm{CH}_{4}$-Anstieg in der Atmosphäre von 35-40 Tg $\mathrm{CH}_{4}$ $\mathrm{a}^{-1}$ (IPCC, 1994). Bei den für die Oxidation des Methans verantwortlichen Mikroorganismen handelt es sich um eine weitgehend unbekannte Gruppe von Bakterien, die fast ausschließlich im Oberboden aktiv ist (HARRIS et al., 1982). Im Unterschied zu den genannten, an Methanquellen lebenden methanotrophen Bakterien handelt es sich im Oberboden um Bakterien mit einer extrem hohen Substrataffinität. Sie verfügen alle über das Enzym Methan-monooxigenase, das eine Oxidation der relativ geringen atmosphärischen Methanmengen ermöglicht (Czepiel et al., 1995, Bender und ConRAD, 1992). Den $\mathrm{CH}_{4}$-oxidierenden Mikroorganismen in den terrestrischen Oberböden wird eine regulierende Funktion im globalen Methankreislauf zugeschrieben (IPCC, 1994). Die Methanoxidierer reagieren auf Veränderungen der Methankonzentration direkt. OJIMA et al. (1993) berichten, daß der Anstieg der atmosphärischen Methankonzentration von 0,7 auf heute 1,7 ppmv zu einer wesentlich besseren $\mathrm{CH}_{4}$-Versorgung der Methanoxidierer geführt hat und sich die $\mathrm{CH}_{4}$-Aufnahmerate aus der Luft überproportional auf $250 \%$ erhöht hat. 


\subsubsection{Prozesse der Oxidation atmosphärischen Methans in Böden}

Im globalen Methankreislauf stellt die biologische Oxidation durch das Schlüsselenzym Methanmonooxigenase (MMO) für Methan eine wichtige Senke dar. Die Oxidation von Methan bis hin zum Kohlendioxid dient den methanotrophen Bakterien zur Energiegewinnung und Substratversorgung (Abb. 2-4). Ein verwandter mikrobieller Oxidationsprozeß ist die Oxidation von $\mathrm{NH}_{3}$ zu Hydroxylamin durch die Ammoniummonooxygenase (AMO) (BEDARD und KNOWLES, 1989).

Sowohl die MMO als auch die AMO zeichnen sich durch eine relativ breite Substratspezifität aus. Aufgrund einer ähnlichen Molekülstruktur vermögen sie sowohl Ammoniak wie auch Ammonium zu oxidieren. Die Konzentration der Substrate und die Affinität der Enzyme zu ihrem Substrat sind entscheidend für ihren Umsatz. Nach Ammoniumzufuhr kommt es somit zur Konkurrenz um aktive Zentren von der MMO wie auch von AMO. Dieses wird als Ursache für die Reduktion der Methanoxidation nach Ammoniumzufuhr angesehen (MOSIER et al., 1991; HüTSCH et al., 1994; SCHNELL und KING, 1994; DUNFIELD und KNOWLES, 1995; BORKEN, 1996).

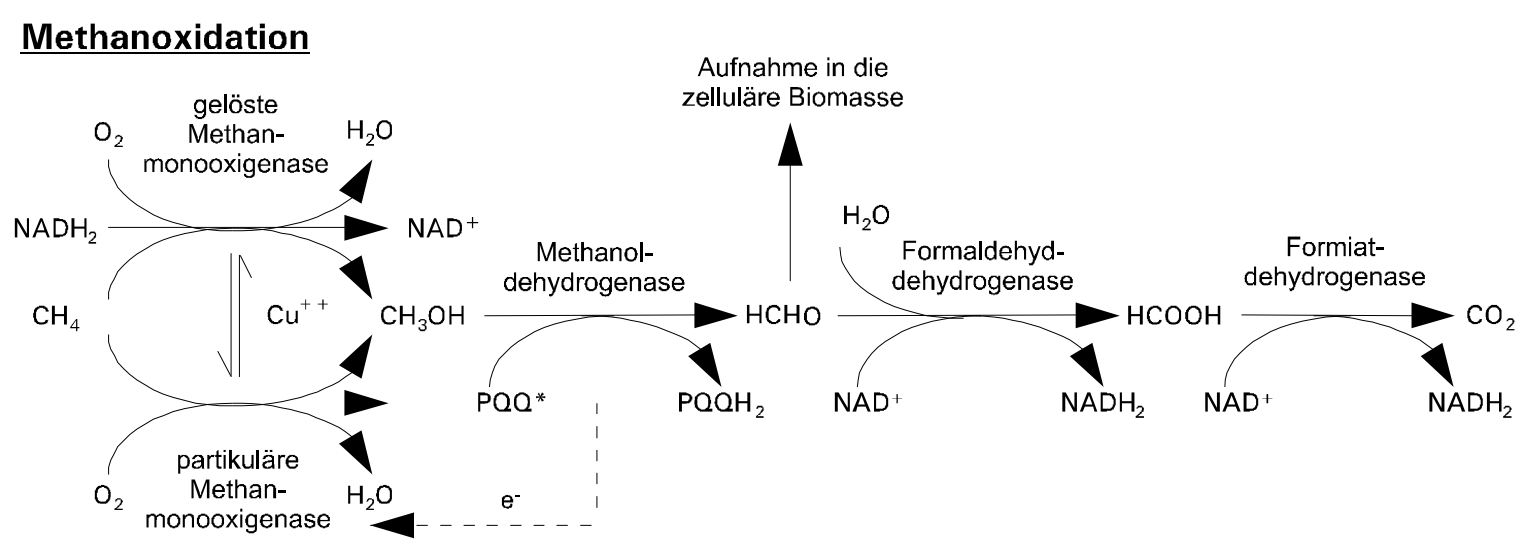

\section{Ammoniumoxidation}

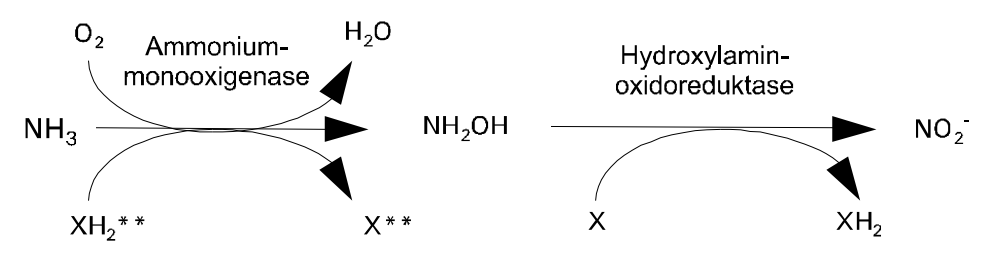

Abb. 2-4: Reaktionsfolge der Methan- und Ammoniumoxidation (nach BEDARD und KNOWLES, 1989). 
In einer Studie von SCHIMEL et al. (1993) werden die wichtigsten Steuergrößen der Methanoxidation in hierachischer Reihenfolge dargestellt. In dieser wie auch in anderen Arbeiten wird als Haupteinflußfaktor der Methanoxidation die Gasdiffusion von der Atmosphäre zum Ort der Konsumenten betrachtet (STRIEGEL, 1993). Die Gasdiffusion in den Boden hinein wird vor allen Dingen vom luftführenden Porenvolumen und der Tortuosität des Bodens bestimmt. Sie ändert sich mit dem Wassergehalt des Bodens, mit Bodenbearbeitungsmaßnahmen, sowie durch Bioturbation. Der dominierende Einfluß des Wassers auf die Gasdiffusion wird durch die Tatsache verdeutlicht, daß Methan in Wasser einen ca. $10^{4}$-fach höheren Diffusionskoeffizienten gegenüber demjenigen in Luft aufweist (MARRERO und MASON, 1972). Deshalb wird in vielen Arbeiten der Wassergehalt des Bodens als Haupteinflußgröße der Methanaufnahme genannt (STEUDLER et al., 1989, WHALEN et al., 1990, CzEPIEL et al., 1995). Die Gaspermeabilität des Bodens ist ein weiterer Parameter, der die Methanzufuhr aus der Atmosphäre zum Oxidationsort bestimmt. Diese wird besonders von kontinuierlichen Poren bestimmt, die sowohl auf biogenem Wege von Wurzeln oder Regenwürmern wie auch auf physikalischem Wege (Polyedergefüge) gebildet werden. Neben dem Bodengefüge hat auch die Bodentextur (Korngrößenzusammensetzung) einen Einfluß auf die Methandiffusion. Auf sandigen, skeletthaltigen Böden werden meist höhere Methanaufnahmeraten gemessen (DörR et al., 1993).

Der Einfluß der Bodentemperatur auf die Aktivität der methanotrophen Bakterien spielt aufgrund der Substratlimitierung eine untergeordnete Rolle (KING und AdAMSON, 1992; CRILL et al., 1994; BORKEN, 1996). Indirekt wirkt sich die Temperatur jedoch über eine Veränderung der Evapotranspiration auf die Methandiffusion aus. Eine eindeutige Aussage über die steuernde Wirkung von biologischen oder chemischen Bodenparametern ist nur eingeschränkt möglich, da deren Effekte durch physikalische Transportprozesse überlagert werden. DUNFIELD et al. (1993) fanden, daß das Optimum der Methanoxidation bei pH 5-7 liegt, woraus abgeleitet wurde, daß die Methanoxidierer nur teilweise an saure Bedingungen adaptiert sind. Auch BORKEN und BRUMME (1997) zeigten auf versauerten Waldböden einen insgesamt positiven Effekt der pH-Erhöhung auf die Methanoxidation.

\subsection{3 $\mathrm{CH}_{4}$-Aufnahme terrestrischer Böden}

Die Jahresflüsse der Methanoxidation verschiedener terrestrischer Standorte unterscheiden sich mit jährlichen Oxidationsraten zwischen 0,1 bis $15,2 \mathrm{~kg} \mathrm{CH}_{4} \mathrm{ha}^{-1}$ beträchtlich voneinander. Die höchsten Flüsse wurden in Wäldern mit sandigen bis steinigen Böden gemessen (STEUDLER et al., 1989; CRILL, 1991; BOWDEN et al., 1993; DOBBIE und SMITH, 1996), die niedrigsten Raten sind typisch für 
landwirtschaftlich genutzte Flächen (Flessa et al., 1995; DoBBIE und SMITH, 1996; MCDONALD et al., 1996). Mit Flußraten zwischen 0,1 und 2,0 $\mathrm{kg} \mathrm{CH}_{4} \mathrm{ha}^{-1} \mathrm{a}^{-1}$ liegt die Variation der verschiedenen Agrarflächen (Grünland, Ackerland etc.) bedeutend niedriger als die der Waldökosysteme, bei denen eine Variationsbreite von 0,49 und $15,2 \mathrm{~kg} \mathrm{CH}_{4} \mathrm{ha}^{-1} \mathrm{a}^{-1}$ gefunden wurde (MOSIER et al., 1991; LESSARD et al., 1994; Ambus und CHRISTENSEN, 1995; DobBie et al., 1996; STEUDLER et al., 1989).

Die Umwandlung unkultivierter Flächen bzw. naturnaher Ökosysteme in landwirtschaftlich genutzte Flächen hat im Mittel zu einer $40 \%$ igen Reduktion der Senkenstärke terrestrischer Böden für atmosphärisches Methan geführt (OJIMA, 1993). Eine Abnahme der Methanoxidation im Zusammenhang mit einer veränderten Landnutzung wurde sowohl für tropische (KELLER et al., 1990) als auch für gemäßigte Klimabreiten (DOBBIE et al., 1996) nachgewiesen. Die Vielzahl an Faktoren, die eine Veränderung der $\mathrm{CH}_{4}$-Oxidation bewirkt, ist jedoch bis heute wegen ihrer Komplexität noch nicht vollständig bekannt (KING und ADAMSON, 1992). DOBBIE et al. (1996) zeigten, daß durch die Umwandlung von Waldflächen in landwirtschaftliche Flächen allein im temperaten Klimabereich die Methanoxidation um ca. 2,3 $\mathrm{Tg} \mathrm{CH}_{4} \mathrm{a}^{-1}$ zurückging. PRIEME et al. (1997) zeigten, daß durch die Inkulturnahme die $\mathrm{CH}_{4}$-Oxidationsleistung langfristig reduziert wird. Nach Wiederaufforstung landwirtschaftlich genutzter Böden sind annähernd 100 Jahre nötig, bis das natürliche Oxidationspotential erreicht wird.

Natürliche terrestrische Ökosysteme zeigen gegenüber landwirtschaftlich genutzten Flächen im allgemeinen höhere Aufnahmeraten. Landwirtschaftliche Weizenflächen wiesen im Vergleich zu ungestörtem Grasland eine um $75 \%$ geringere jährliche Methanaufnahme auf (OJIMA et al., 1993). Die Inkulturnahme von natürlichen Ökosystemen ist jedoch fast immer mit zusätzlichen Stickstoffeinträgen verbunden, so daß es sich hierbei häufig um einen Kombinationseffekt handelt, der auch durch Düngungsmaßnahmen beeinflußt wird. Der Stickstoffeinsatz in der Landwirtschaft wird für eine Reduzierung der Senkenstärke verantwortlich gemacht (STEUDLER et al., 1989; Mosıer et al., 1991; HüTSCH et al., 1994). In den Waldökosystemen Mitteleuropas kommt es seit einigen Jahrzehnten zu erhöhten indirekten Stickstoffeinträgen aus der Landwirtschaft (BEESE und MATZNER, 1986). OJIMA et al. (1993) induzierten durch Stickstoffzufuhr auch in natürlichen Ökosystemen einen Rückgang der Oxidation atmosphärischen Methans. Verglichen mit der Landnutzungsänderung ist der unmittelbare N-Düngungseffekt in der Regel geringer (STEUdLeR et al., 1989; SteUdLeR et al., 1996; Mosier et al., 1991; HÜTSCH et al., 1994).

Als eine weitere Ursache für eine geringere Methanoxidation in Böden von Agrarökosystemen gelten die bewirtschaftungsbedingten Bodenverdichtungen. 
HANSEN et al. (1993) fanden auf einem Ackerboden nach Bodenverdichtungen eine Reduktion der Methanaufnahme um 50 \%. Auch der anthropogene Säureeintrag in die Böden kann die Senkenstärke für atmosphärisches Methan reduzieren (BORKEN und BRUMME, 1997).

Die anthropogene Reduktion der Methansenke terrestrischer Böden wird global auf ca. 3 - $4 \mathrm{Tg} \mathrm{CH}_{4} \mathrm{a}^{-1}$ geschätzt (POTTER et al., 1996). Es kann bisher keine Aussage darüber getroffen werden, ob durch eine extensivere landwirtschaftliche Nutzung der Böden die Methanoxidation langfristig wieder ansteigt. Viele wissenschaftliche Untersuchungen deuten jedoch darauf hin, daß eine Verminderung der Stickstoffdüngung langfristig zu einer Erhöhung der $\mathrm{CH}_{4}$-Oxidation führt. 


\section{3 Über die Bedeutung des Winterrapsanbaus und der N-Düngung für die $\mathrm{N}_{2}$ O-und $\mathrm{CH}_{4}$-Flüsse eines Gley-Auenbodens}

\subsection{Einleitung}

1997 wurden in der Bundesrepublik Deutschland ca. 914.000 ha WRaps angebaut (StATISTISCHES BundesAmT, 1997), neben der Nahrungsmittelproduktion fand ein großer Teil des Raps als nachwachsender Rohstoff zur Produktion von Rapsölmethylester (RME) Verwendung. RME wird als Kraftstoffersatz eingesetzt, um unter anderem die Emissionen der treibhauswirksamen Gase $\mathrm{CO}_{2}, \mathrm{~N}_{2} \mathrm{O}$ und $\mathrm{CH}_{4}$, die bei der Verbrennung fossiler Energieträger auftreten, zu reduzieren. $\mathrm{CO}_{2}$ ist in der Atmosphäre in einer Konzentration von ca. 355 ppmv enthalten, $\mathrm{N}_{2} \mathrm{O}$ von ca. 311 ppbv und $\mathrm{CH}_{4}$ von 1,74 ppmv. Das bei der Nutzung von RME freiwerdende $\mathrm{CO}_{2}$ wird als klimaneutral gewertet, da es zuvor der Atmosphäre über die Photosynthese entzogen wurde. Dagegen werde entsprechend der Ökostudie zum RME (UBA, 1993) die Erdatmosphäre zusätzlich mit $\mathrm{N}_{2} \mathrm{O}$ belastet, da es beim Anbau von WRaps $\mathrm{zu}$ erhöhten $\mathrm{N}_{2} \mathrm{O}$-Feldemissionen käme. Bezüglich der Bewertung der $\mathrm{N}_{2} \mathrm{O}$ Emissionen wird im Rahmen des UBA-Gutachtens (UBA 1993) betont, daß die Schätzungen über zusätzliche $\mathrm{N}_{2} \mathrm{O}$-Emissionen, die speziell dem Anbau von WRaps zuzuordnen sind, noch sehr unsicher seien. In der vorliegenden Literatur gibt es keinen Hinweis auf Langzeituntersuchungen, in denen der Einfluß des Winterraps auf die $\mathrm{N}_{2} \mathrm{O}$-Emissionen nachgewiesen wurde. FINDLAY und MCKENNEY (1979) und VAN CleEMPUT et al. (1992) konnten zwar fruchtartspezifische $\mathrm{N}_{2} \mathrm{O}$-Emissionen nachweisen (Klee, Luzerne, Mais u.a.), jedoch leiteten sie diese Erkenntnis lediglich aus Kurzzeituntersuchungen ab.

BOUWMAN (1990) und VAN KESSEL et al. (1993) machten deutlich, daß mit steigender $\mathrm{N}$-Düngung meistens auch mit einer Zunahme der $\mathrm{N}_{2} \mathrm{O}$-Emissionen zu rechnen sei; ein eventueller Einfluß der angebauten Frucht wurde hierbei jedoch nicht berücksichtigt. Schon EICHNER (1990) weist jedoch in seinen Untersuchungen darauf hin, daß die Höhe der $\mathrm{N}_{2} \mathrm{O}$-Emissionen auch von der Art des $\mathrm{N}$-Düngers abhängen kann.

Die Bildung und Emission von $\mathrm{N}_{2} \mathrm{O}$ ist auf die Tätigkeit von Denitrifikanten und Nitrifikanten im Boden zurückzuführen (FIRESTONE et al., 1979; ROBERTSON, 1989). Diese Mikroorganismen konkurrieren mit den angebauten Pflanzen um den Stickstoff in der Bodenlösung (SCHACHTSCHABEL et al., 1989). Ihre Aktivität wird durch Einflußgrößen, wie z.B. dem Bodenwassergehalt, der Temperatur des Bodens wie auch der Bodenart bestimmt (BouWMAN, 1990). Da nach DAVIDSON (1991) das Ausmaß der Lachgasproduktion wiederum von der Menge des zur Verfügung 
stehenden Stickstoffs abhängt, wird der Einfluß der Vegetation auf die $\mathrm{N}_{2} \mathrm{O}$ Emissionen eines Standortes deutlich.

Verschiedene Fruchtarten weisen unterschiedliche Stickstoffaneignungsvermögen auf. LICKFETT (1993) berichtet, daß sich WRaps durch ein erhebliches NAufnahmevermögen auszeichnet. Es müssen jedoch auch die unterschiedlichen Vorfruchteffekte verschiedener Fruchtarten im Hinblick auf die einsetzende NMineralisierung der verbliebenen Erntereste berücksichtigt werden. ENGEL und MANGSTL (1988) sowie BECKER (1991) berichten von hohen $\mathrm{N}_{\min }$-Gehalten (ca. $95 \mathrm{~kg}$ $\mathrm{ha}^{-1} 90 \mathrm{~cm}^{-1}$ ) im Herbst nach dem Anbau von Raps oder Ackerbohnen. LICKFETT (1993) berichtet, daß es gerade nach dem Anbau von WRaps gegenüber dem Getreide im Herbst und Winter unabhängig von der Produktionsintensität zu hohen $\mathrm{N}_{\text {min }}$-Gehalten im Boden (> $100 \mathrm{~kg} \mathrm{ha}^{-1} 90 \mathrm{~cm}^{-1}$ ) kommt. Es ist also zu vermuten, daß unabhängig von der Höhe der N-Düngung besonders nach Raps erhöhte $\mathrm{N}_{2} \mathrm{O}$ Emissionen auftreten.

Vor diesem Hintergrund erscheint es problematisch, die $\mathrm{N}_{2} \mathrm{O}$-Emissionen der Produktionsflächen wie üblich anhand grober Schätzungen vereinfachend zu ermitteln, indem von einem konstanten Emissionsfaktor ausgegangen wird. BouWmaN (1994) schätzt die $\mathrm{N}_{2} \mathrm{O}-$ Emissionen landwirtschaftlich genutzter Böden in den gemäßigten Zonen auf ca. 1,25\% des eingetragenen Stickstoffs.

Aerobe Böden terrestrischer Ökosysteme stellen die einzige biologische Senke für atmosphärisches $\mathrm{CH}_{4}$ dar (CICERONE und OREMLAND, 1988; WATSON et al., 1992). Landwirtschaftlich beeinflußte Flächen zeigten bei einer Erhöhung der N-Zufuhr keine nennenswerte Reduktion der $\mathrm{CH}_{4}$-Oxidationsleistungen (FLESSA et al., 1995; BORKEN, 1996). Es wird hingegen davon ausgegangen, daß die Umwandlung naturnaher Ökosysteme in landwirtschaftlich genutzte Flächen in Verbindung mit höheren N-Einträgen im Mittel zu einer $40 \%$ igen Reduktion der Methanaufnahmerate dieser Böden führt (OJIMA et al., 1993). Über eine Reduktion der Methanaufnahmeraten des Bodens durch den Anbau von WRaps gibt die Literatur jedoch keine Auskunft. Als wichtigster Einflußfaktor der Methanoxidation gilt die Gaspermeabilität des Bodens (SCHIMEL et al., 1993; STRIEGEL, 1993), die vom Wassergehalt des Bodens maßgeblich beeinflußt wird. Die $\mathrm{CH}_{4}$-Oxidation in gut durchlüfteten Böden erstreckt sich von $0-20 \mathrm{~cm}$ unter GOF und nimmt mit zunehmender Tiefe ab (CRILL, 1991; AdAMSEN und KING, 1993; TYLER et al., 1994).

Um Kenntnisse über die Spurengasflüsse beim Anbau von Winterraps als nachwachsenden Rohstoff zu gewinnen, wurden anhand eines Feldversuchs über einen Zeitraum von 2 Jahren wöchentlich Spurengasmessungen in der Fruchtfolge WRaps-WWeizen-WGerste durchgeführt. Parallel dazu wurden Bodenparameter wie 
Bodenfeuchte, Bodentemperatur und Bodennitratgehalt aufgenommen, um deren Einflüsse auf die $\mathrm{N}_{2} \mathrm{O}$ - und $\mathrm{CH}_{4}$-Spurengasflüsse des Standortes herauszuarbeiten. Weiterhin wurde geklärt, ob es hinsichtlich der Spurengasflüsse fruchtartspezifische Unterschiede gab und inwieweit sich eine Halbierung der N-Düngung auf diese auswirkte.

Die Klimawirksamkeit der Produktion einzelner Fruchtarten der Fruchtfolge WRapsWWeizen-WGerste wird in dieser Arbeit unter Berücksichtigung einer differenzierten $\mathrm{N}$-Düngung abgeleitet und verglichen. Es wird versucht, das $\mathrm{CO}_{2}$ Einsparungspotential von Raps als nachwachsendem Rohstoff im Hinblick auf eine mögliche Reduzierung des anthropogen bedingten Treibhauseffektes unter Einbeziehung des gesamten Lebensweges bis hin zur Produktion von Rapsölmethylester (RME) abzuschätzen. 


\subsection{Material und Methoden}

\subsubsection{Versuchsstandort}

\subsubsection{Geographische Lage, Klima und Witterung}

Der Feldversuch wurde auf dem Flurstück „Leinekamp“ des Versuchsgutes Reinshof (Gö, 51³1'48' Breite; 956’39" Länge) der Universität Göttingen durchgeführt (Abb. 3-1), das ca. 2 km südlich von Göttingen gelegen ist.

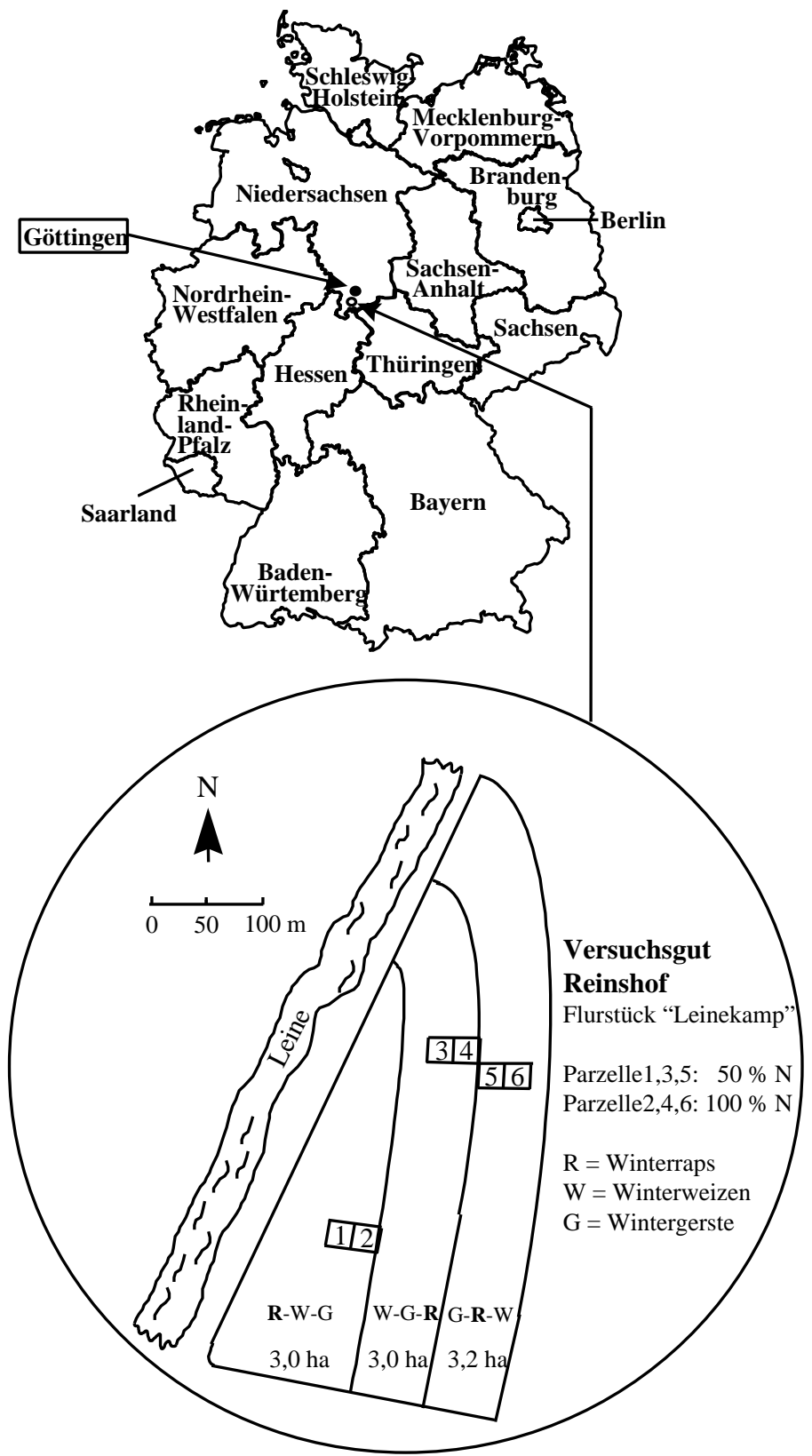

Abb. 3-1: $\quad$ Lage des Versuchsstandortes und Anlage der Versuchsparzellen in der Zeit von März 1995 bis Februar 1997. 
Der Standort liegt am östlichen Ufer der Leine auf $153 \mathrm{~m}$ über NN und weist im 40jährigen Mittel eine Lufttemperatur von $8,6^{\circ} \mathrm{C}$ und eine jährliche Niederschlagssumme von $635 \mathrm{~mm}$ auf (Deutscher Wetterdienst Göttingen). Für die Erfassung der Wetterdaten stand auf dem Versuchsgelände eine Meßstation (FMA, Fa. Lambrecht, Göttingen) zur Verfügung. Unter Standardbedingungen wurden in stündlicher Auflösung die Lufttemperatur (2 $\mathrm{m}$ Höhe über GOF) und der Niederschlag (0,5 m über GOF) ermittelt (Abb. 3-2).

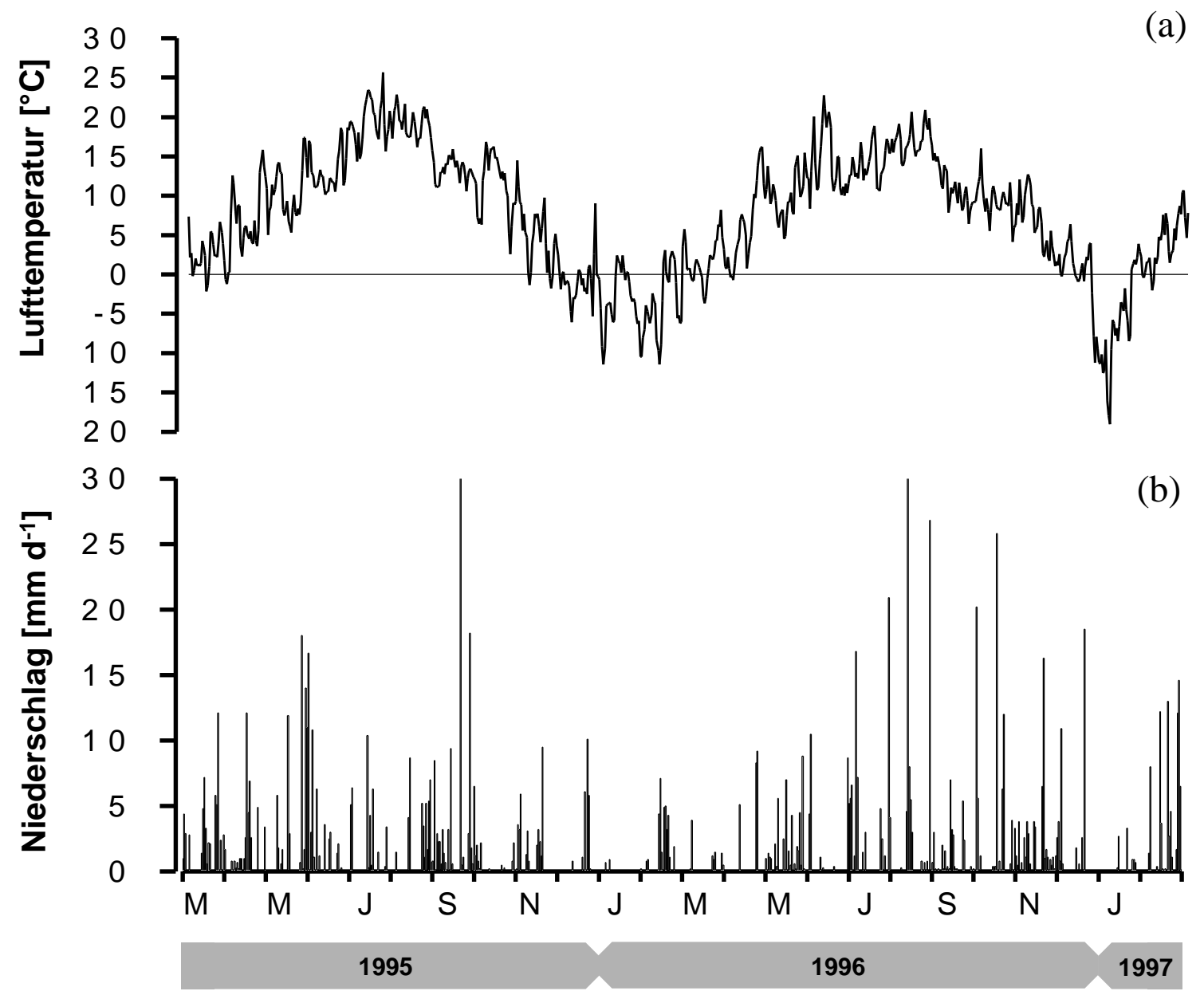

Abb. 3-2: $\quad$ Mittlere Tagestemperaturen in $2 \mathrm{~m}$ über GOF (a) und tägliche Niederschlagsmengen (b) des Standorts für den Versuchszeitraum 01. März 1995 bis 28. Februar 1997.

Hieraus ergaben sich die im Folgenden aufgeführten mittleren Lufttemperaturen und die mittleren Niederschläge für die Jahre 1995 und 1996:

$\begin{array}{lccc} & 1995 & 1996 & \text { langjähriges Mittel } \\ \text { mittlere Lufttemperatur }\left[{ }^{\circ} \mathrm{C}\right] & 8,5 & 6,8 & 8,6 \\ \text { Summe Niederschlag }[\mathrm{mm}] & 620 & 513 & 635\end{array}$


Das Jahr 1996 zeichnete sich im langjährigen Vergleich durch relativ geringe Niederschläge aus, während das Jahr 1996 eine relativ hohe mittlere Jahrestemperatur aufwies. Parallel zu den wöchentlichen Spurengasmessungen (Kap. 3.2.3.1) wurden der Luftdruck und die bodennahe Lufttemperatur bestimmt, um die Spurengasflüsse berechnen zu können (vgl. Kap. 3.2.3.3). Gleichzeitig wurde auch die Bodentemperatur in $2,5 \mathrm{~cm}$ Tiefe gemessen, da diese als eine der steuernden Einflußgrößen der $\mathrm{N}_{2} \mathrm{O}$-Emissionen gilt (vgl. Kap. 2.1).

\subsubsection{Physikalische und chemische Eigenschaften des Bodens}

Der Gley-Auenboden des Versuchsstandortes besteht aus stark bis mittel schluffigem Ton mit einer hohen bis sehr hohen nutzbaren Feldkapazität von 170225 mm (TEIWES, 1997). Der Grundwasserflurabstand schwankte im Versuchszeitraum zwischen 1,80 und 1,00 m. Innerhalb der oberen $8 \mathrm{dm}$ des Profils war der Grundwassereinfluß durch Eisenfleckigkeit zu erkennen. Die wichtigsten bodenchemischen und -physikalischen Kenngrößen, die während des Versuchs-

Tab. 3-1: $\quad$ Physikalische und chemische Eigenschaften des Versuchsbodens

\begin{tabular}{|c|c|c|c|c|c|c|c|c|c|c|c|}
\hline \multirow[t]{3}{*}{$\begin{array}{l}\text { Par- } \\
\text { zelle }\end{array}$} & \multirow[t]{3}{*}{$\begin{array}{l}\text { Versuchs- } \\
\text { glied }\end{array}$} & \multirow{3}{*}{$\begin{array}{c}\text { Tiefe } \\
\text { [cm u. GOF] }\end{array}$} & \multirow{3}{*}{ Horizont } & \multicolumn{3}{|c|}{$\begin{array}{l}\text { Korngrößen- } \\
\text { verteilung }\end{array}$} & \multirow{3}{*}{$\begin{array}{c}d_{B} \\
{\left[g^{-3} c^{-3}\right]}\end{array}$} & \multirow{3}{*}{$\begin{array}{c}\mathrm{pH} \\
\left(\mathrm{CaCl}_{2}\right)\end{array}$} & \multirow{3}{*}{$\begin{array}{c}\mathrm{CaCO}_{3} \\
\text { [\%] }\end{array}$} & \multirow{3}{*}{$\begin{array}{c}\mathbf{N}_{\mathbf{t}} \\
\text { [\%] }\end{array}$} & \multirow{3}{*}{$\begin{array}{l}\mathrm{C}_{\text {org }} \\
\text { [\%] }\end{array}$} \\
\hline & & & & $\mathbf{S}$ & $\mathbf{U}$ & $\mathbf{T}$ & & & & & \\
\hline & & & & [\%] & [\%] & [\%] & & & & & \\
\hline 2 & R-W-G & $5-10$ & $A p$ * & 4,7 & 64,9 & 30,4 & 1,24 & 7,5 & 4,55 & 0,19 & 1,55 \\
\hline 2 & & $30-35$ & $A h^{*}$ & 4,3 & 65,3 & 30,4 & 1,33 & 7,6 & 5,01 & 0,16 & 1,49 \\
\hline 2 & & $145-150$ & Go-Gr * & 1,1 & 46,0 & 52,9 & 1,51 & 7,6 & 10,10 & 0,11 & 1,42 \\
\hline 4 & W-G-R & $5-10$ & Ap & 4,7 & 70,2 & 25,1 & 1,35 & 7,6 & 5,00 & 0,16 & 1,49 \\
\hline 4 & & $30-35$ & Ah & 4,2 & 77,4 & 18,4 & 1,49 & 7,8 & 5,33 & 0,15 & 1,41 \\
\hline 6 & G-R-W & $5-10$ & $A p$ & 4,6 & 60,2 & 35,2 & 1,33 & 7,5 & 2,88 & 0,21 & 1,57 \\
\hline 6 & & $30-35$ & $\mathrm{Ah}$ & 4,9 & 62,3 & 32,8 & 1,42 & 7,6 & 3,24 & 0,18 & 1,48 \\
\hline & Gras & $5-10$ & $A p$ & 3,6 & 68,8 & 27,6 & 1,38 & 7,1 & 3,76 & 0,18 & 1,46 \\
\hline & & $30-35$ & $\mathrm{Ah}$ & 3,4 & 68,5 & 28,1 & 1,39 & 7,4 & 4,13 & 0,16 & 1,47 \\
\hline
\end{tabular}

* Diese Horizonte wurden auch für den Laborversuch (Kapitel 4) verwendet. 
zeitraumes im Juli 1995 erhoben wurden, sind in Tabelle 3-1 zusammengefaßt. Die Lagerungsdichte $\left(d_{B}\right)$ des Ap- wie auch des Ah-Horizontes wurden 2 mal jährlich bestimmt und über den Versuchszeitraum gemittelt; die Lagerungsdichte des Go-GrHorizontes wurde im April 1996 einmalig nach Aushub einer Bodengrube zur Entnahme von ungestörten Bodensäulen (vgl. Kap. 4.2.1) bestimmt. Hinsichtlich der Versorgung mit Phosphor, Kalium und Magnesium befanden sich die Versuchsflächen in der Gehaltsstufe C, deshalb mußten laut Empfehlung der Offizialberatung diese Grundnährstoffe nur nach Entzug gedüngt werden.

\subsubsection{Versuchsvarianten}

Der Feldversuch mit der Fruchtfolge Winterraps - Winterweizen - Wintergerste wies zwei N-Düngungsstufen auf: $100 \%$ und $50 \%$ der durch die Offizialberatung gegebenen N-Düngungsempfehlung. Als N-Dünger wurde Kalkammonsalpeter (27\% N) verwendet. Als Vergleichsvariante diente eine mehrjährig ungedüngte Grasfläche, die im Abstand von ca. $200 \mathrm{~m}$ zu den Versuchsflächen auf gleichartigem Boden gelegen war. Die Versuchsglieder wurden auf einer Fläche von jeweils

Tab. 3-2: Fruchtarten und Düngungsstufen des Feldversuches

\begin{tabular}{ccc|cc|cc}
\hline \multirow{2}{*}{$\begin{array}{c}\text { Par- } \\
\text { zelle }\end{array}$} & $\begin{array}{c}\text { Versuchs- } \\
\text { glied }\end{array}$ & Hauptfrucht & $\begin{array}{c}\text { N-Düngung } \\
{\left[\mathbf{k g ~ h a}^{-1}\right]^{*}}\end{array}$ & Hauptfrucht & $\begin{array}{c}\text { N-Düngung } \\
{\left[\mathbf{k g ~ h a}^{-1}\right]^{*}}\end{array}$ & $\begin{array}{c}\text { 1996/97 } \\
\text { Hauptfrucht }\end{array}$ \\
\hline 1 & R-W-G & WRa & 90 & WW & 55 & WG \\
2 & R-W-G & WRa & 182 & WW & 110 & WG \\
\hline 3 & W-G-R & WW & 92 & WG & 65 & WRa \\
4 & W-G-R & WW & 185 & WG & 130 & WRa \\
\hline 5 & G-R-W & WG & 87 & WRa & 66 & WW \\
6 & G-R-W & WG & 175 & WRa & 133 & WW \\
\hline & Gras & Gras & 0 & Gras & 0 & Gras \\
\hline
\end{tabular}

$\mathrm{WRa}=$ Winterraps, Sorte Lirajet

$\mathrm{WW}=$ Winterweizen, Sorte Xanthos

WG = Wintergerste, Sorte Sympax (1994/95) und Sorte Venus (1995/96 und 96/97)

* Die N-Düngung wurde unter Berücksichtigung von $\mathbf{N}_{\min }$ im Frühjahr in 0-30 $\mathrm{cm}$ Bodentiefe durchgeführt 
$48 \mathrm{~m} \times 25 \mathrm{~m}$ ohne Wiederholung angelegt, da auf diesen die Spurengasmessungen in 4-facher Wiederholung durchgeführt wurden (vgl. Kap. 3.2.3.1). Die Versuchsanlage gewährleistete, daß alle Fruchtarten und Düngungsstufen in beiden Versuchsjahren vorhanden waren (Tabelle 3-2).

\subsubsection{Untersuchungsmethoden}

\subsubsection{Gewinnung der Gasproben im Feld}

Die Gewinnung der auf $\mathrm{N}_{2} \mathrm{O}, \mathrm{CH}_{4}$, und $\mathrm{CO}_{2}$ zu untersuchenden Gasproben erfolgte im Feldversuch von März 1995 bis Februar 1997 einmal wöchentlich mittels geschlossener Bodenhauben in vierfacher Wiederholung, die kurzzeitig auf die Bodenoberfläche aufgesetzt wurden („closed-chamber-method“) (Abb. 3-3). Die Hauben wurden auf fest installierte Bodensockel aufgesteckt, die zu Versuchsbeginn $15-20 \mathrm{~cm}$ tief in den Boden gedrückt wurden. Die Bodensockel wurden nur in der Zeit von Ernte- und Bodenbearbeitungsmaßnahmen kurzfristig entfernt. Sie dienten zur Verhinderung eines seitlichen Nachströmens von Frischluft in die Hauben während der Anreicherungszeit. Die gewählte Installationstiefe dieser Sockel verringerte auch die von HEALY et al. (1996) beschriebene laterale Gasdiffusion im Boden während der Anreicherungszeit von 30 bis 90 Minuten.

Je Haube wurde zu Beginn, nach der Hälfte und zum Ende der Gasanreicherungszeit aus der Haubenatmosphäre eine Gasprobe entnommen. Die Gasemissions- bzw. Gasaufnahmeraten wurden aus der Konzentrationsänderung von $\mathrm{N}_{2} \mathrm{O}, \mathrm{CH}_{4}$ und $\mathrm{CO}_{2}$ in der Haubenatmosphäre während des Beprobungszeitraumes errechnet. Die Flußraten für die Meßtermine wurden durch das arithmetische Mittel beschrieben. Die verwendeten zylindrischen PVC-Hauben waren lichtundurchlässig, um eine Erwärmung des abgedeckten Bodens zu vermeiden und deckten eine Bodenfläche von $1900 \mathrm{~cm}^{2}$ ab. Um im Haubenbereich weitgehend natürliche Feldbedingungen zu erhalten, wurden die Pflanzen während der Anreicherungsphase in die Hauben eingeschlossen. Durch Distanzringe konnte die Haubenhöhe dem Pflanzenbestand bis zu einer Pflanzenlänge von $80 \mathrm{~cm}$ angepaßt werden. Die Rapspflanzen mußten daher mit einer zuvor in den Hauben installierten Stützhilfe verflochten werden. Bei Getreide war dieses nicht erforderlich. Die Gasanreicherungszeit variierte in Abhängigkeit von der Anzahl der Distanzringe sowie der erwarteten $\mathrm{N}_{2} \mathrm{O}$-Emissionsaktivität zwischen 30 und 90 Minuten. Mit jedem Distanzring wurde das Anreicherungsvolumen vergrößert; somit mußte im allgemeinen auch die Anreicherungszeit erhöht werden, um bei gleichbleibender Grundfläche eine detektierbare Gasanreicherung zu gewährleisten. Die 
Gasprobenahme aus den verschlossenen Hauben erfolgte mittels eines batteriebetriebenen Gasprobennehmers (LOFTFIELD et al., 1997), auf dem eine zuvor evakuierte Gasprobenflaschen mit einem Volumen von $100 \mathrm{ml}$ aufgesteckt wurde. Ein Gasprobenehmer ermöglichte vor der jeweiligen Probenahme ein Spülen der Ansaugleitung mit der Haubenluft und eine Kontrolle auf Dichtigkeit der evakuierten Probenflaschen.

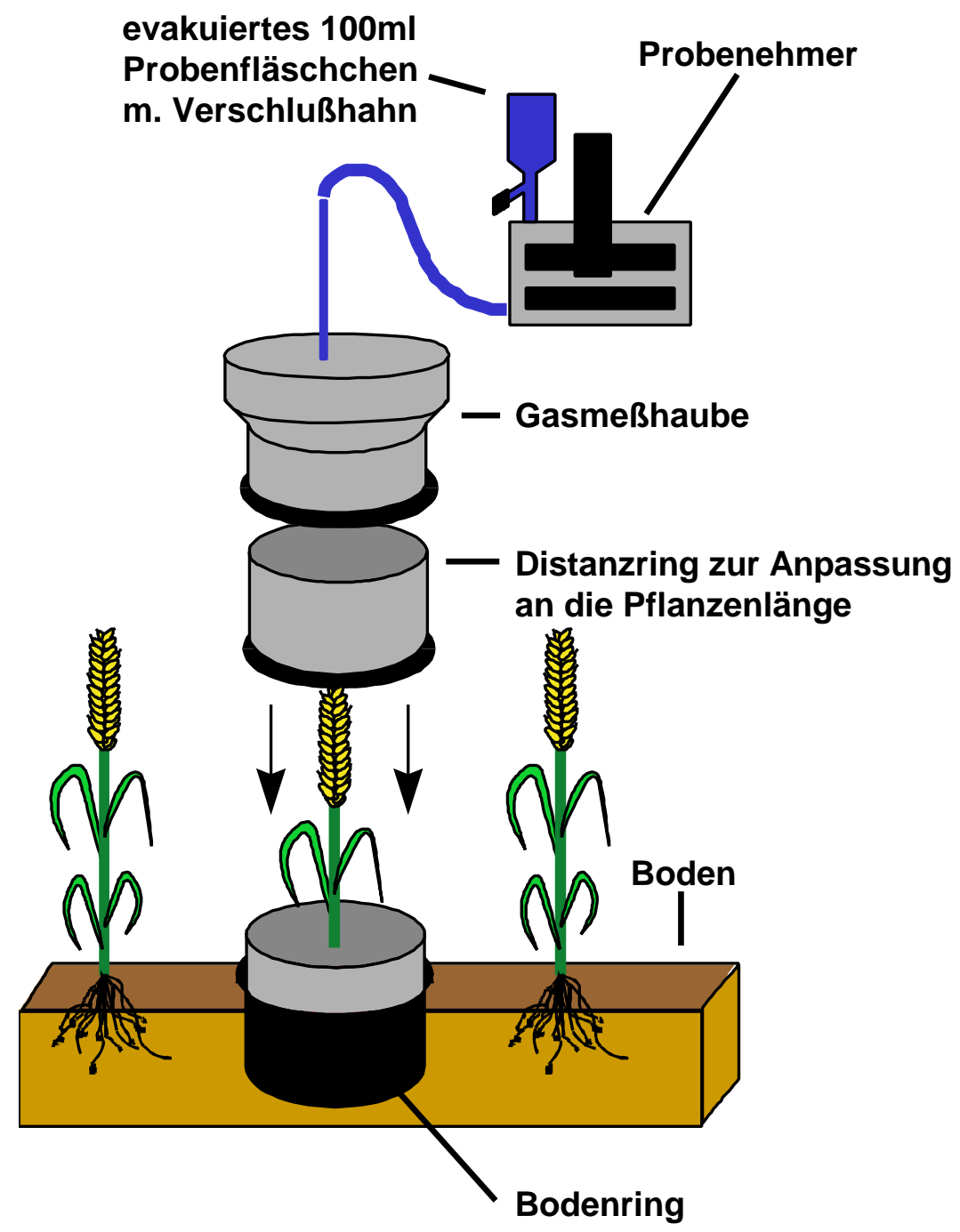

Abb. 3-3: $\quad$ Schema der Gasprobenahme im Feld (,closed-chamber-method“) 


\subsubsection{Spurengasanalytik}

Nach der Probenahme wurden die Gasproben innerhalb von 3 Tagen im Labor mit einem Gasanalysensystem, wie es von LOFTFIELD et al. (1997) detailliert beschrieben wurde, analysiert. Dieses System bestand aus einem Autosampler für Gassammelflaschen und einem Gaschromatographen (Carlo Erba, Typ GC 6000), der mit einem ${ }^{63} \mathrm{Ni}$-Elektroneneinfangdetektor (ECD) sowie einem Flammenionisationsdetektor (FID) ausgestattet war. Der ECD wurde zur Messung der $\mathrm{CO}_{2-}$ wie auch $\mathrm{N}_{2} \mathrm{O}$-Konzentrationen benötigt und zeigte nach 28 Messwiederholungen für die ambienten Eichgaskonzentrationen für $\mathrm{CO}_{2}$ (350 ppmv) und $\mathrm{N}_{2} \mathrm{O}$ (330 ppbv) einen relativen Fehler von $\pm 0,5 \%$ bzw. $\pm 0,9 \%$ (BORKEN, 1996). Der FID wurde zur Messung der Methankonzentration benötigt und zeigte für die ambiente Eichgaskonzentration von $\mathrm{CH}_{4}(1900$ ppbv) einen relativen Meßfehler von $\pm 0,1 \%$ (Tab. 3-3).

Tab. 3-3: Reproduzierbarkeit der Gasanalyse. Angegeben sind das Mittel $\bar{x}$, die Standardabweichung s und der Variationskoeffizient Vk der Integrale I sowie der relative Fehler (n = 28) (BORKEN, 1996).

\begin{tabular}{rrrcc}
\hline Gasart & $\overline{\mathrm{x}}$ & $\mathbf{s}$ & Vk [\%] & relativer Fehler [\%] \\
\hline $\mathrm{CO}_{2}$ & 357,1 & 4,6 & 1,3 & 0,5 \\
$\mathrm{~N}_{2} \mathrm{O}$ & 353,9 & 7,8 & 2,2 & 0,9 \\
$\mathrm{CH}_{4}$ & 1449,6 & 4,0 & 0,3 & 0,1 \\
\hline
\end{tabular}

\subsubsection{Berechnung der Spurengasflüsse}

Die Errechnung der Gasflußraten für $\mathrm{CO}_{2}, \mathrm{~N}_{2} \mathrm{O}$ bzw. $\mathrm{CH}_{4}$ erfolgte anhand der Steigung der Regressionsgeraden (Änderung der Gaskonzentration in der Haube über die Zeit) nach den folgenden Gleichungen:

$$
\begin{array}{lll}
\mathrm{FCO}_{2} & =\frac{d c}{d t} * 10^{-6} * 60 *\left(\frac{\mathrm{Mi} * 10^{3} * \mathrm{VH}}{\mathrm{Mv} * \mathrm{~A}}\right) * R & {\left[\mathrm{mg} \mathrm{m}^{-2} \mathrm{~h}^{-1}\right]} \\
\mathrm{FN}_{2} \mathrm{O} \text { bzw. } \mathrm{CH}_{4}=\frac{d c}{d t} * 10^{-9} * 60 *\left(\frac{\mathrm{Mi} * 10^{6} * \mathrm{VH}}{\mathrm{Mv} * \mathrm{~A}}\right) * R & {\left[\mu \mathrm{g} \mathrm{m}^{-2} \mathrm{~h}^{-1}\right]}
\end{array}
$$




\begin{tabular}{|c|c|c|c|c|}
\hline $\mathrm{dc} / \mathrm{d}$ & $=$ & \multicolumn{3}{|c|}{$\begin{array}{l}\left.\text { Konzentrationsänderung der Gase } \mathrm{CO}_{2} \text { [ppmv } \min ^{-1}\right] \text { bzw. } \mathrm{N}_{2} \mathrm{O} \\
\left.\text { und } \mathrm{CH}_{4} \text { [ppbv } \text { min }^{-1}\right]\end{array}$} \\
\hline \multirow[t]{3}{*}{$\mathrm{Mi}$} & $=$ & Molmasse & $\mathrm{CO}_{2}-\mathrm{C}$ & $12\left[\mathrm{~g} \mathrm{~mol}^{-1}\right]$ \\
\hline & & & $\mathrm{N}_{2} \mathrm{O}-\mathrm{N}$ & $28\left[\mathrm{~g} \mathrm{~mol}^{-1}\right]$ \\
\hline & & & $\mathrm{CH}_{4}-\mathrm{C}$ & $12\left[\mathrm{~g} \mathrm{~mol}^{-1}\right]$ \\
\hline $\mathrm{V}_{\mathrm{H}}$ & $=$ & \multicolumn{3}{|c|}{ Volumen der Haube [l] } \\
\hline \multirow[t]{3}{*}{ Mv } & $=$ & Molvolumen & $\mathrm{CO}_{2}$ & $22,26\left[\mathrm{Imol}^{-1}\right]$ \\
\hline & & & $\mathrm{N}_{2} \mathrm{O}$ & $22,25\left[\mid \mathrm{mol}^{-1}\right]$ \\
\hline & & & $\mathrm{CH}_{4}$ & $22,36\left[\mid \mathrm{mol}^{-1}\right]$ \\
\hline A & $=$ & \multicolumn{3}{|c|}{ Bodenoberfläche der Haube $\left[\mathrm{m}^{2}\right]$} \\
\hline $\mathrm{R}$ & $=$ & \multicolumn{3}{|c|}{ Reduktionsfaktor } \\
\hline
\end{tabular}

Die Reduktion des Gasvolumens auf den Normalzustand $\left(0^{\circ} \mathrm{C}\right.$ und $\left.1013 \mathrm{hPa}\right)$ ist nach den Gasgesetzen für ein ideales Gas durch den Reduktionsfaktor $(R)$ gegeben:

$$
R=\frac{p_{0}}{p a^{*}\left(1+0,00367 * T_{a}\right)}
$$

$\mathrm{p}_{0} \quad=\quad$ Normaldruck $[\mathrm{hPa}]$

$\mathrm{p}_{\mathrm{a}}=$ Luftdruck zur Zeit der Probenahme [hPa]

$\mathrm{T}_{\mathrm{a}}=$ Lufttemperatur in Bodennähe zur Zeit der Probenahme $\left[{ }^{\circ} \mathrm{C}\right]$

Luftdruck sowie Lufttemperatur wurden zu den jeweiligen Probenahmeterminen vor Ort ermittelt.

\subsubsection{Bodenphysikalische Untersuchungen}

\subsection{Bodenwassergehalt}

Die Bodenwassergehalte W\% wr wurden gravimetrisch durch Trocknung des Bodens (105 ${ }^{\circ} \mathrm{C}, 24$ Stunden) bestimmt und nach HARTGE (1991) berechnet:

$$
W \% \operatorname{tr}=\frac{B d_{n}-B d_{t r}}{B d_{t r}} \times 100
$$


$\mathrm{Bd}_{\mathrm{n}} \quad=$ nasser Boden $[\mathrm{g}]$

$\mathrm{Bd}_{\mathrm{tr}} \quad=$ trockener Boden $[\mathrm{g}]$

\subsection{Lagerungsdichte und Gesamtporenvolumen}

Die Lagerungsdichte $\left(\mathrm{d}_{\mathrm{B}}\left[\mathrm{g} \mathrm{cm}^{-3}\right]\right)$ wurde an ungestörten Stechzylinderproben $\left(257 \mathrm{~cm}^{3}\right.$ ) ermittelt (HARTGE, 1992) und das Gesamtporenvolumen (GPV) nach HARTGE (1991) wie folgt berechnet:

$$
\mathrm{GPV}=1-\frac{\mathrm{d}_{\mathrm{B}}}{\mathrm{d}_{\mathrm{F}}} \times 100
$$

$\mathrm{d}_{\mathrm{B}} \quad=$ Lagerungsdichte des Bodens $\left[\mathrm{g} \mathrm{cm}^{-3}\right]$

$\mathrm{d}_{\mathrm{F}} \quad=$ Dichte der festen Bodenbestandteile $\left[\mathrm{g} \mathrm{cm}^{-3}\right]$

(Quarz $=2,65 \mathrm{~g} \mathrm{~cm}^{-3}$ )

\subsection{Volumetrischer Wassergehalt und volumetrischer Luftgehalt}

Der volumetrische Wassergehalt (Vol.H2O) des Bodens wurde nach HARTGE (1992) berechnet:

Vol $_{H_{2} \mathrm{O}}=\frac{B d_{n}-B d_{t r}}{B d_{t r}} \times \frac{d_{B}}{d_{W}} 100 \quad[\%]$

$\mathrm{Bd}_{\mathrm{n}}=$ nasser Boden [g]

$\mathrm{Bd}_{\mathrm{t}}=$ trockener Boden [g]

$\mathrm{d}_{\mathrm{W}} \quad=$ Dichte des Wassers $\left[\mathrm{g} \mathrm{cm}^{-3}\right]$ 
Der volumetrische Luftgehalt (Vol.Luft) des Bodens ergibt sich aus:

Vol.Luft $=$ GPV - Vol. ${ }_{2} \mathrm{O}[\%]$

\subsection{Anteil des volumetrischen Wassergehaltes am Gesamt- porenvolumen des Bodens}

Der relative Anteil wassergefüllter Poren am Gesamtporenvolumen (GPV) des Bodens („water-filled-pore-space“, WFPS) wurde aus dem Vol. H2O und dem GPV des Bodens berechnet:

$$
\text { WFPS }=\frac{\mathrm{Vol}_{\mathrm{H}_{2} \mathrm{O}}}{\mathrm{GPV}} \times 100[\%]
$$

\subsection{Porengrößenverteilung und Korngrößenfraktionierung}

Die Porengrößenverteilung wurde anhand der Wassergehalte bei verschiedenen Wasserspannungen errechnet (SCHLICHTING et al., 1995). Die Korngrößenfraktionen des Bodens wurden durch Sieben bzw. Sedimentationsverfahren bestimmt (SCHLICHTING et al., 1995).

\subsubsection{Bodenchemische Methoden}

\subsubsection{1 pH-Wert}

Die Bestimmung des $\mathrm{pH}$-Wertes erfolgte mittels $\mathrm{pH}$-Elektrode in einer $0,01 \mathrm{M} \mathrm{CaCl}_{2}-$ Lösung.

\subsection{Gesamt-C- und Gesamt-N-Gehalt des Bodens}

Gesamt-C $\left(\mathrm{C}_{\mathrm{t}}\right)$ und Gesamt-N $\left(\mathrm{N}_{\mathrm{t}}\right)$ wurden durch Elementaranalyse (C/N-Analysator NA 1500, Fa. CARLO ERBA) bestimmt. 


\subsection{Carbonat-C}

Das im Boden enthaltene $\mathrm{CaCO}_{3}$ wurde nach SCHEIBLER gasvolumetrisch ermittelt (SCHLICHTING und BLUME, 1966).

\subsection{Mineralischer Stickstoff}

Die Bestimmung der $\mathrm{N}_{\text {min }}$-Gehalte wurde in feldfeuchten Bodenproben mit 0,01 M $\mathrm{CaCl}_{2}$-Lösung (HouBA et al., 1986) durchgeführt. Nitrat und Ammonium wurden mit Hilfe eines Continuous-Flow-Autoanalyzers (SFAS 5100, Fa. Skalar) photometrisch bestimmt.

\subsubsection{Bewertung der Treibhauswirksamkeit}

Die im Rahmen dieser Arbeit analytisch ermittelten $\mathrm{N}_{2} \mathrm{O}$-,,Feld“-Emissionen (vgl. 3.2.3.1) bei der Produktion von WRaps, WWeizen und WGerste stellen nur einen Teil der durch den Anbau bedingten zusätzlichen klimarelevanten Emissionen dar. Um den Anteil dieser Emissionen an der gesamten Treibhauswirksamkeit beim Anbau dieser Früchte zu beschreiben und die durch den Anbau bedingten zusätzlichen Gesamtemissionen der verschiedenen Früchte miteinander vergleichen zu können, wurde zusätzlich auf Datenmaterial aus der Literatur zurückgegriffen. Dieses gilt im besonderen bei der Abschätzung des $\mathrm{CO}_{2}$-Einsparungspotentials von Raps als nachwachsenden Rohstoff, bei der der gesamte Lebensweg des Raps bis hin zur Produktion von Rapsölmethylester (RME) berücksichtigt wurde.

Bilanziert wurden die Treibhausgase $\mathrm{N}_{2} \mathrm{O}$ und $\mathrm{CH}_{4}$. Die Klimawirksamkeit dieser Gase wurde mit den GWP-Werten (Global Warming Potential, IPCC, 1994) beschrieben, die die relative Treibhauswirksamkeit bezogen auf $\mathrm{CO}_{2}$ wiedergeben (Tab. 3-4). Bei der Berechnung der GWP-Werte wurde ein Integrationszeitraum von 100 Jahren zugrundegelegt (vgl. IPCC, 1994), die mittlere atmosphärische Verweildauer der Gase wurde berücksichtigt. Die Emissionen von $\mathrm{N}_{2} \mathrm{O}$ und $\mathrm{CH}_{4}$ ließen sich anschließend als $\mathrm{CO}_{2}$-Äquivalente darstellen und konnten aufsummiert und verglichen werden. 
Tab. 3-4: Relative Treibhauspotentiale (GWP-Werte) der betrachteten klimawirksamen Gase bezogen auf die gleiche Masse $\mathrm{CO}_{2}$ und einem Zeitraum von 100 Jahren (IPCC, 1994).

Treibhausgas

Treibhauspotential

(GWP-Werte)

\begin{tabular}{ccc}
\hline Kohlendioxid & $\mathrm{CO}_{2}$ & 1 \\
Distickstoffoxid & $\mathrm{N}_{2} \mathrm{O}$ & 320 \\
Methan & $\mathrm{CH}_{4}$ & 24,5 \\
\hline
\end{tabular}

Um den Treibhauseffekt durch die Produktion der 3 Fruchtarten WRaps, WWeizen und WGerste zu beschreiben, wurden folgende Emissionen als $\mathrm{CO}_{2}$-Äquivalente ausgedrückt und addiert:

\section{Emissionen biologischen Ursprungs:}

- die direkten $\mathrm{N}_{2} \mathrm{O}$-Emissionen

- die indirekten $\mathrm{N}_{2} \mathrm{O}$-Emissionen, die aus dem Austrag des Stickstoffs in benachbarte Systeme resultieren

\section{Klimawirksame Emissionen energetischen Ursprungs:}

- die $\mathrm{CO}_{2}-, \mathrm{N}_{2} \mathrm{O}$ - und $\mathrm{CH}_{4}$-Emissionen, die im Zuge der Bereitstellung von Düngeund Pflanzenschutzmitteln auftraten

- die $\mathrm{CO}_{2}$-Emissionen durch den Verbrauch und die Bereitstellung von Dieselkraftstoff für sämtliche Bewirtschaftungsmaßnahmen

Um fruchtartspezifische direkte $\mathbf{N}_{2} \mathrm{O}$-Emissionen berechnen zu können, wurde eine zeitliche Zuordnung der im Feld ermittelten Emissionen zu den Fruchtarten vorgenommen; die Emissionsraten der entsprechenden Zeiträume wurden anschließend kumuliert (vgl. Kap. 3.2.5). FLESSA et al. (1995) bemerken, daß eine Beschränkung der fruchtspezifischen Emissionen auf die Vegetationszeit einer Fruchtart unter unseren Klimabedingungen für eine verläßliche Abschätzung der Jahresflüsse nicht geeignet sei. Im Rahmen dieser Arbeit wurde deshalb der Zeitraum nach der Ernte bis zum Vegetationsbeginn im folgenden Frühjahr der Vorfrucht zugeordnet. Hieraus ergab sich für die einzelnen Fruchtarten ein Bilanzierungszeitraum vom 1. März des Erntejahres bis zum 28./29. Februar des 
Folgejahres. Diese Aufteilung hatte zur Folge, daß bei einer Winterung als nachgestellter Hauptfrucht ein Teil inrer Vegetationszeit, nämlich die Austriebsperiode im Herbst, der Vorfrucht zugeordnet wurde. Der Bilanzzeitraum der Winterung begann somit erst im Frühjahr (ab März) mit den Düngungsmaßnahmen.

Die in die Bilanzierung aufgenommene Emission errechnete sich aus der fruchtspezifischen Emission abzüglich der Basisemission der ungedüngten Grasfläche im gleichen Zeitraum.

Die indirekten $\mathbf{N}_{2} \mathrm{O}$-Emissionen, die nur aus dem Austrag von Stickstoff in benachbarte Systeme resultieren, wurden nicht erfaßt. Es wurde daher entsprechend den Angaben des IPCC (1996) von einer indirekten Emission in Höhe von $0,75 \%$ der applizierten Stickstoffmenge ausgegangen.

Die energetischen Emissionen durch die Bereitstellung von Dünge- und Pflanzenschutzmittel wurden anhand von Literaturdaten abgeschätzt (KALTSCHMITT und REINHARD, 1997). Die zugrundegelegten mittleren Emissionsfaktoren für die aufgewandten Produktionsmittel sind in Tabelle 3-5 aufgeführt.

Tab. 3-5: Energetische Emissionen von $\mathrm{CO}_{2}, \mathrm{~N}_{2} \mathrm{O}$ und $\mathrm{CH}_{4}$ durch die Bereitstellung von Produktionsmitteln sowie durch die Bereitstellung und Verbrennung von Dieselkraftstoff im Zuge der Bewirtschaftung der Produktionsfläche (HYDRO AGRI, 1993; KALTSCHMITT und REINHARDT, 1997).

\begin{tabular}{llll}
\hline Produktionsmittel & \multicolumn{3}{c}{ Emissionen } \\
& $\begin{array}{l}\mathrm{CO}_{2} \\
{[\mathrm{~g}]}\end{array}$ & $\begin{array}{l}\mathbf{N}_{\mathbf{2}} \mathrm{O} \\
{[\mathrm{g}]}\end{array}$ & $\begin{array}{l}\mathbf{C H}_{4} \\
{[\mathrm{~g}]}\end{array}$ \\
\hline Stickstoffdünger $(1 \mathrm{~kg} \mathrm{~N})$ & 2468 & 9,63 & 0,45 \\
Phosphor-Dünger $\left(1 \mathrm{~kg} \mathrm{P} \mathrm{O}_{5}\right)$ & 700 & 0,042 & 0,023 \\
Kalium-Dünger $\left(1 \mathrm{~kg} \mathrm{~K} \mathrm{~K}_{2} \mathrm{O}\right)$ & 453 & 0,0094 & 0,021 \\
Pflanzenschutzmittel $(1 \mathrm{~kg}$ Wirkstoff $)$ & 4921 & 1,51 & 0,18 \\
Dieselkraftstoff $(1 \mathrm{~kg})$ & 3490 & & \\
\hline
\end{tabular}

Die energetischen Emissionen im Zuge der Bewirtschaftungsmaßnahmen der Fruchtfolge wurden über den Dieselkraftstoffverbrauch (Ackerschlepper/Erntemaschinen) erfaßt. Neben der direkten $\mathrm{CO}_{2}$-Emission aus der Verbrennung des Diesels wurden auch die indirekten Emissionen durch die Bereitstellung des Kraftstoffs (Exploration, Raffinerieverarbeitung, Transport) berücksichtigt. Bei den 
Berechnungen wurde eine mittlere $\mathrm{CO}_{2}$-Emission von $3,49 \mathrm{~kg}$ je verbrauchtem Kilogramm Dieselkraftstoff zugrundegelegt (HYDRO AGRI, 1993) (Tab. 3-5). Der Kraftstoffverbrauch für alle ackerbaulichen Maßnahmen wurde anhand von Literaturdaten (HYDRO AGRI, 1993) ermittelt. Es wurde davon ausgegangen, daß der für die Grundbodenbearbeitung (Grubber- und Pflugarbeiten), die Saatbettbereitung und die Aussaat (Kreiselegge und Drillmaschine) eingesetzte $110 \mathrm{~kW}$-Schlepper einen mittleren Kraftstoffverbrauch von $13 \mathrm{~kg} \mathrm{~h}^{-1}$ aufwies. Für den Pflegeschlepper (Pflegemaßnahmen und Abfuhr von Erntegut (3 km Entfernung Feld - Hof)) mit 55 $\mathrm{kW}$ wurde ein mittlerer Verbrauch von $7 \mathrm{~kg} \mathrm{~h}^{-1}$ und für den Mähdrescher von $35 \mathrm{~kg} \mathrm{~h}^{-1}$ veranschlagt.

Die Fixierung von Kohlendioxid wurde bei der durchgeführten Bilanzierung als klimaneutral gewertet, da sowohl bei der energetischen Nutzung als auch beim biologischen Abbau durch die Biomasse das $\mathrm{CO}_{2}$, welches zuvor über die Photosynthese der Atmosphäre entzogen wurde, wieder emittiert wird. Diese Betrachtung basiert auf der Annahme, daß der Vorrat an organischer Substanz im Boden konstant bleibt.

\subsubsection{Statistische Auswertung}

Die Datenkalkulationen und Berechnungen von Mittelwert und Standardabweichung wurden mit Excel 7.0 (Microsoft) durchgeführt. Für weiterführende statistische Verrechnungen wurde das Programmpaket Sigma Stat/Sigma Plot (Jandel Scientific $\mathrm{GmbH}$, Erkrath) verwendet. Die Gasflußraten und die parallel erhobenen Steuergrößen der Feldversuche wurden mit dem Kolmogorow-Smirnov-Test auf Normalverteilung geprüft. Bei den normal verteilten bzw. nach Transformation normal verteilen Daten wurden Pearsonsche Korrelationskoeffizienten berechnet. Ließen sich die Datensätze auch durch Transformation nicht normal verteilen, wurden Spearmansche Rangsummen-Korrelationen berechnet. Um die Wirkungsstärke einzelner, den Gasfluß bestimmender Parameter zu quantifizieren, wurde bei normal verteilten Datensätzen eine multiple schrittweise Regressionsanalyse durchgeführt. Um in den Versuchsvarianten, wie z.B. den beiden N-Stufen oder den Ackerflächen bzw. dem Grasland, die Mittelwerte der Meßgrößen auf statistische Unterschiede zu prüfen, wurde unter der Annahme von nicht normalverteilten Daten der Mann-Whitney-Rangsummentest durchgeführt. Beim Vergleich von mehr als zwei Varianten wurde der Student-Newman-Keuls-Test angewendet. 


\subsection{Ergebnisse}

Die Höhe der $\mathrm{N}_{2} \mathrm{O}$-Emissionen eines Bodens wird durch biologische, physikalische und chemische Einflußfaktoren bestimmt, da diese sowohl direkt als auch indirekt auf die $\mathrm{N}_{2} \mathrm{O}$-Entstehungsprozesse der Nitrifikation und Denitrifikation wirken (vgl. Kap. 2.1.2). Als die wichtigsten Steuergrößen für die $\mathrm{N}_{2} \mathrm{O}$-Abgaberaten eines Standortes werden jedoch immer wieder die Bodentemperatur, das wassergefüllte Porenvolumen und die $\mathrm{N}_{\min }$-Gehalte des Bodens genannt (ROBERTSON, G.P., 1989; GRANLI und BocKMANN, 1994). Diese Einflußfaktoren werden in der Literatur auch im Hinblick auf die Höhe der Methanoxidation kontrovers diskutiert (STEUDLER et al., 1989; KING und ADAMSON, 1992; BORKEN, 1996). Auch in der vorliegenden Arbeit soll der Einfluß dieser drei Bodenparameter auf die Spurengasflüsse herausgearbeitet werden. Vor der Darstellung der Spurengasflüsse werden im folgenden zunächst die zeitlichen Verläufe der genannten Einflußfaktoren dargestellt, die parallel zu den Spurengasmessungen erhoben wurden.

Die Probenahmen erfolgten wöchentlich von Mitte März 1995 bis Ende Februar 1997. Da die Untersuchungen in der WRaps - Fruchtfolge in den 3 Varianten R (=WRaps) -W (=WWeizen) -G (=WGerste), W-G-R und G-R-W parallel durchgeführt wurden, war ein Vergleich der Fruchtarten in jedem Jahr möglich. In den Ergebnisdarstellungen wurden sowohl die aufgenommenen Bodenparameter als auch die Spurengasflüsse jeweils für einen Jahreszeitraum vom 1. März - 28./29. Februar dargestellt. Desweiteren wurde differenziert nach Sommer- und Winterhalbjahr. Als Sommerhalbjahr wurde hierbei die Zeit vom 01. März bis 31. August festgelegt und deckt den Zeitraum von der ersten Frühjahrsdüngung bis zur Ernte ab. Es beinhaltet also die Hauptwachstumsphase der jeweiligen Frucht. Das entsprechende Winterhalbjahr (01. September bis 28./29. Februar) umfaßt im wesentlichen die Bestellung und die Winterruhe der Früchte und des Bodens bis zum Beginn des Frühjahrswachstums.

\subsubsection{Zeitlicher Verlauf der Bodenparameter}

\subsubsection{Bodentemperatur und wassergefüllte Porenvolumina}

\section{Bodentemperatur}

Die in Abb. 3-4 und 3-5 dargestellten Bodentemperaturen wurden parallel zu den Spurengasmessungen jeweils zwischen 8 und $14 \mathrm{Uhr}$ aufgenommen. Im Mittel der vier Prüfvarianten lag die durchschnittliche Bodentemperatur in $2,5 \mathrm{~cm}$ Tiefe 1995/96 bei $10,1^{\circ} \mathrm{C}$ (Abb. 3-4). Mitte August wurde mit knapp $30^{\circ} \mathrm{C}$ ein Temperaturmaximum 
erreicht, die niedrigsten Werte lagen Ende Januar bei $-2,7{ }^{\circ} \mathrm{C}$. Es ist besonders darauf hinzuweisen, daß sich im Winterhalbjahr 1995/96 die Monate November bis Februar durch mittlere Bodentemperaturen um den Gefrierpunkt auszeichneten. Dem Gefrieren des Bodens folgte in regelmäßigen Abständen ein Wiederauftauen bzw. ein Antauen des Bodens.

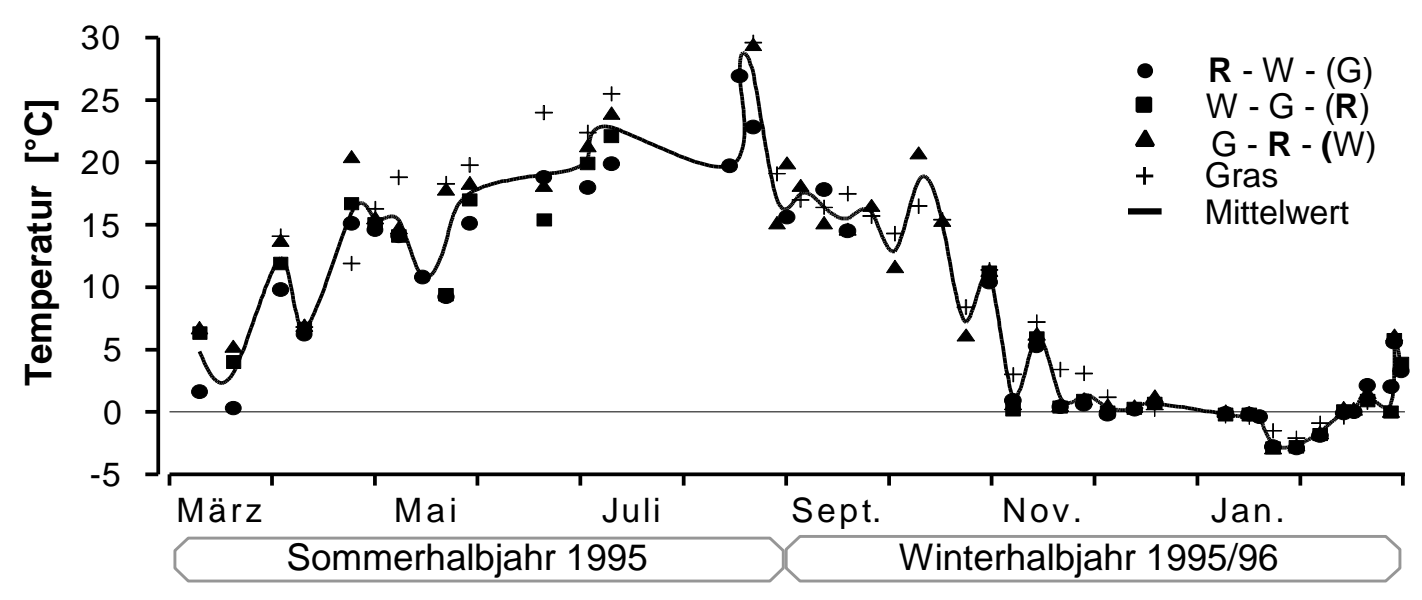

( ) : Frucht des Folgejahres

Abb. 3-4: Bodentemperaturen in 2,5 cm Tiefe zum Zeitpunkt der Gasprobenahme zwischen 8 und 14 Uhr im Zeitraum März 1995 bis Februar 1996.

Im Versuchsjahr 1996/97 (Abb. 3-5) lag die durchschnittliche Bodentemperatur mit $9,6{ }^{\circ} \mathrm{C}$ nur unwesentlich unter der des Vorjahres. Maximale Bodentemperaturen wurden mit durchschnittlich $21{ }^{\circ} \mathrm{C}$ schon Anfang Juni erreicht, minimale Temperaturen um $-2{ }^{\circ} \mathrm{C}$ wurden Anfang Januar gemessen. Das Sommerhalbjahr 1996 zeichnete sich durch einen vergleichsweise kalten Monat März aus, der Boden war bis Mitte des Monats gefroren, erst Anfang April taute der Boden endgültig auf. Im Winterhalbjahr 1996/97 wurden lediglich in der Zeit von Mitte Dezember bis Ende Januar Bodentemperaturen unterhalb des Gefrierpunktes gemessen.

In beiden Versuchsjahren unterschieden sich in den Monaten Oktober bis Februar die Bodentemperaturen der unterschiedlichen Versuchsvarianten nicht voneinander, in den übrigen Monaten kam es hingegen zu stärkeren Abweichungen, die durch unterschiedliche Beschattungen durch den Aufwuchs bzw. mit unterschiedlichen Bodenbearbeitungsmaßnahmen und Bearbeitungsterminen von Juli bis September zu begründen sind. Eine fruchtartspezifische Abweichung von der mittleren Bodentemperatur der Versuchsvarianten ließ sich jedoch nicht erkennen.

Bezüglich des Einflusses der Bodentemperaturen beider Jahre auf die $\mathrm{N}_{2} \mathrm{O}$ Emissionen ist besonders auf die Zeit von November 1995 bis März 1996 
hinzuweisen, da sich diese Zeit durch einen regelmäßigen Wechsel zwischen Frostund Tauphasen auszeichnete. In der Literatur wird häufig auf Frost-Tau induzierte $\mathrm{N}_{2} \mathrm{O}$-Emissionen hingewiesen (FLESSA et al., 1995; KAMP und STEINDL, 1997).

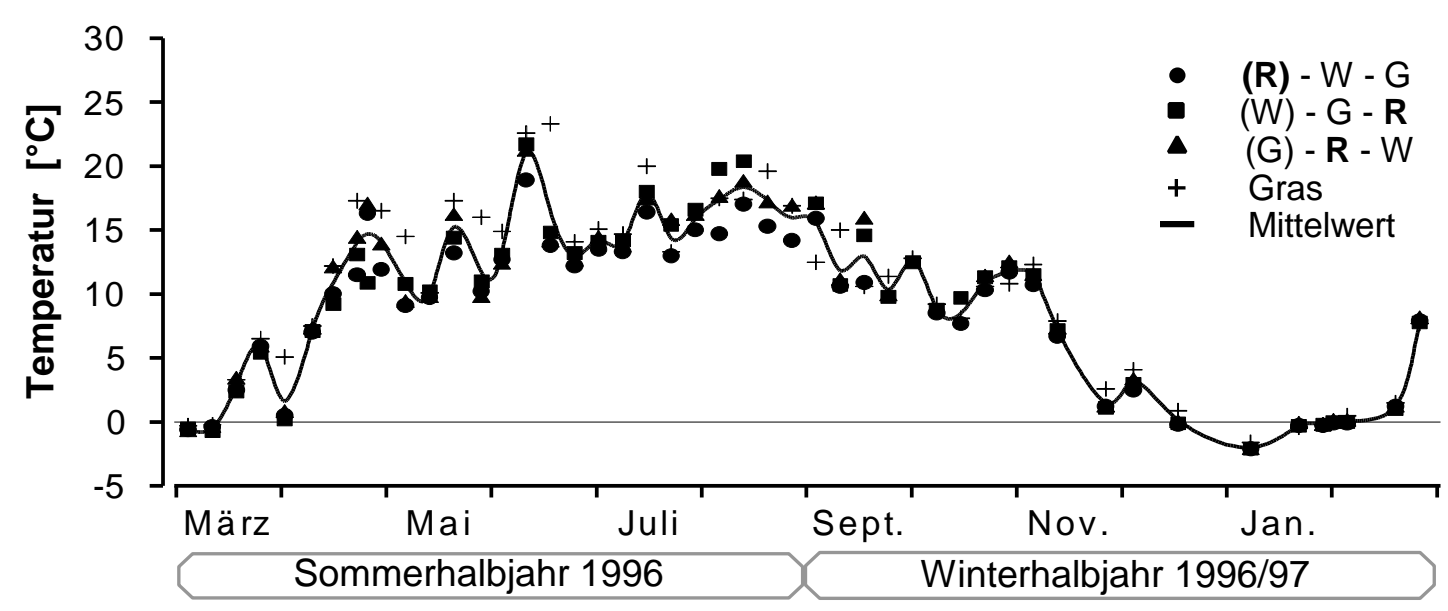

( ) : Frucht des Folgejahres

Abb. 3-5: Bodentemperaturen in 2,5 cm Tiefe zum Zeitpunkt der Gasprobenahme zwischen 8 und 14 Uhr im Zeitraum März 1996 bis Februar 1997.

\section{Wassergefülltes Porenvolumen}

Das wassergefüllte Porenvolumen (WFPS) (Abb. 3-6) des Versuchsstandortes lag im Versuchszeitraum 1995/96 bei durchschnittlich 64,5 \%. Im Mai/Juni stieg das mittlere WFPS nach einer längeren Austrocknungsphase infolge von stärkeren Niederschlägen von $<60 \%$ auf Werte von $>70 \%$ an. Während des Sommerhalbjahres wurden Minima der wassergefüllten Porenvolumina um $44 \%$ Anfang August erreicht. Das WFPS zeigte im Winterhalbjahr 1995/96 (Ende Februar) mit 92,5\% im Mittel über alle Varianten maximale Werte. 


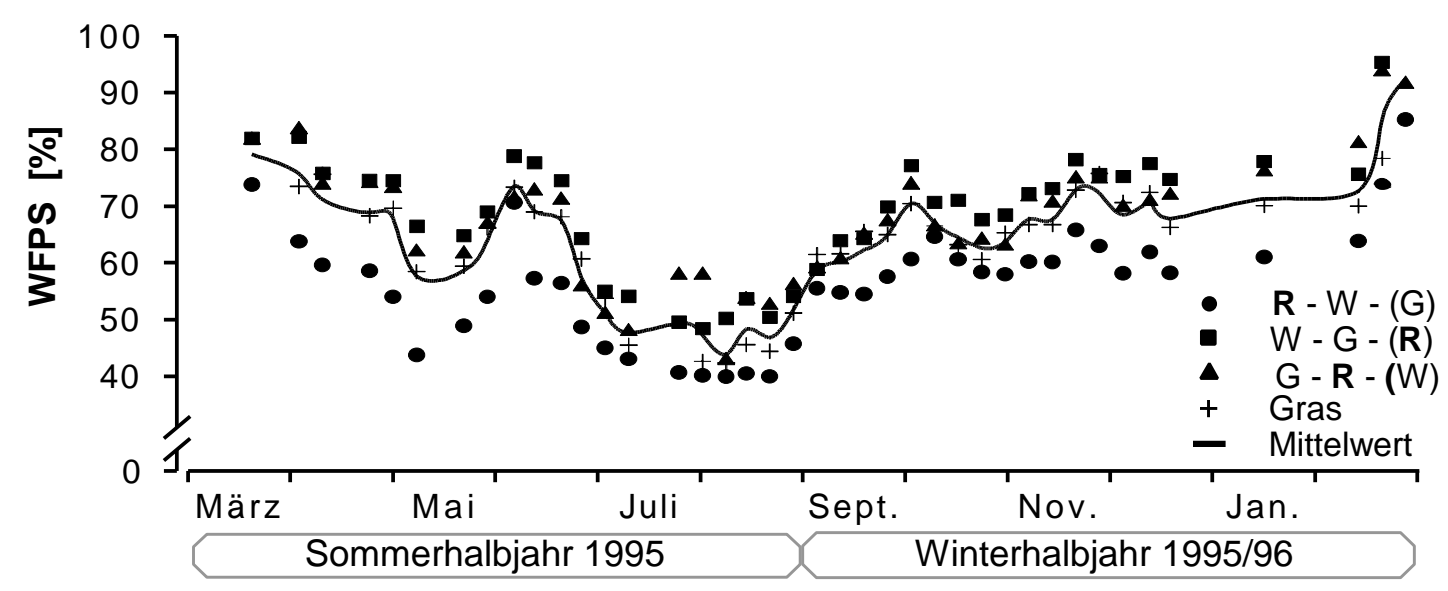

Abb. 3-6: Wassergefüllte Porenvolumina (WFPS) auf den Versuchsflächen im Untersuchungszeitraum März 1995 bis Februar 1996.

1996/97 lag das WFPS im Versuchsmittel mit 65,5\% auf gleichem Niveau wie 1995/96 (Abb. 3-7). Vergleichbar mit dem vorangegangenen Versuchsjahr sank das mittlere WFPS im Sommerhalbjahr auf ca. 43 \% (Juni '96). Im Gegensatz zum Vorjahr stieg aufgrund ausgeprägter Niederschlagsereignisse im Juli 1996 der Anteil der wassergefüllten Porenvolumina vorübergehend auf Werte von $>60 \%$ an. Das maximale WFPS dieses Versuchsjahres lag Ende Januar 1997 mit $85 \%$ im Versuchsmittel jedoch um ca. $7 \%$ unter dem maximalen Wert des Vorjahres.

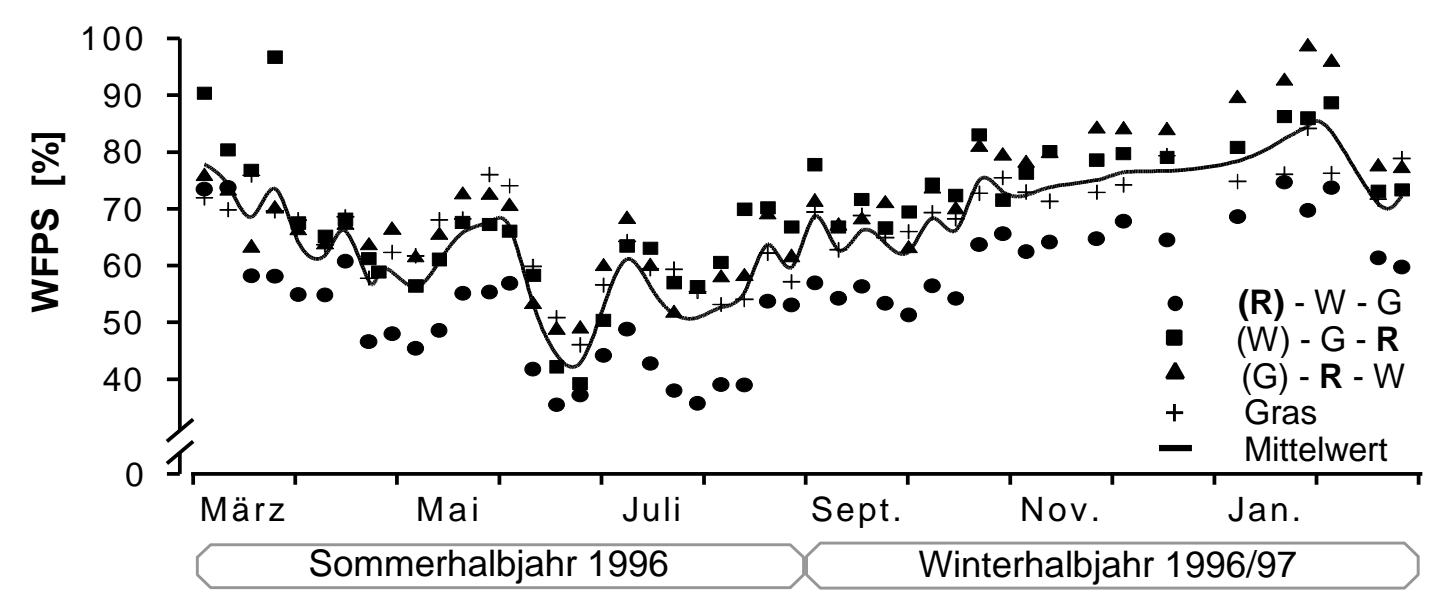

Abb. 3-7: Wassergefüllte Porenvolumina (WFPS) auf den Versuchsflächen im Untersuchungszeitraum März 1996 bis Februar 1997. 
Die Versuchsvariante R-W-G wies über beide Jahre wassergefüllte Porenvolumina auf, die deutlich unter denjenigen des Versuchsmittels lagen. Hierbei muß berücksichtigt werden, daß bei dieser Versuchsvariante die vergleichsweise geringste mittlere Lagerungsdichte des Bodens $\left(1,24 \mathrm{~g} \mathrm{~cm}^{-3}\right)$ in die Berechnung des WFPS einfloß (vgl. Tab. 3-1).

Sowohl im Jahre 1995/96 als auch im Jahre 1996/97 kam es im Mai zu einer kurzfristigen Wiedervernässung des Bodens, während es nach der Ernte im Juli/August jeweils nur zu einem langsamen aber stetigen Anstieg des WFPS bis in den Januar/Februar hinein kam. Gerade nach schneller Wiedervernässung des Bodens infolge starker Niederschläge wird in vielen Arbeiten von erhöhten $\mathrm{N}_{2} \mathrm{O}$ Emissionen berichtet (MOISIER und HUTCHINSON, 1981; FleSSA et al., 1995). Der besonders starke Anstieg des WFPS im Februar 1996 läßt daher hohe $\mathrm{N}_{2} \mathrm{O}$ Emissionen erwarten. Parallel zu einem Anstieg des WFPS sind jedoch sinkende Methanaufnahmeraten zu erwarten. In der Literatur wird von einer negativen Korrelation zwischen dem WFPS und der Methanoxidation berichtet (BOWDEN et al., 1993a).

\subsubsection{Ammonium- und Nitratgehalte des Bodens}

Die $\mathrm{N}_{\min }$-Gehalte wurden lediglich im Ap-Horizont (0-30 cm Bodentiefe) bestimmt, da auch die anderen Bodenparameter nur innerhalb dieses Horizontes ermittelt wurden. Ammonium konnte in $0-30 \mathrm{~cm}$ Bodentiefe auf dem Standort lediglich kurz nach den $\mathrm{N}$-Düngeterminen (KAS) im Boden nachgewiesen werden. Deshalb wurde auf eine Darstellung verzichtet.

In den in Abb. 3-8 und Abb. 3-9 dargestellten Varianten mit $100 \%$ N wurde Stickstoff nach den Empfehlungen der Offizialberatung der Landwirtschaftskammer Hannover ausgebracht. Es handelte sich hierbei also um keine N-Überdüngung, wie sie häufig angewandt wird, um Wirkungen deutlicher zu machen. In den beiden Versuchsjahren führte eine Reduzierung dieser Stickstoffdüngung in der Rotation zu keiner entsprechenden Reduzierung der Bodennitratgehalte in $0-30 \mathrm{~cm}$. In den Varianten (R-W-G - $100 \%$, W-G-R - $100 \%$, G-R-W -100\%) zeigten sich im Vergleich zu den N-reduzierten Varianten (50\%) lediglich direkt nach den N-Düngungen vorübergehend höhere Nitratgehalte. Auch frühere Untersuchungen auf dem Versuchsstandort zeigten, daß eine Reduzierung der N-Düngung bei WRaps, WWeizen und WGerste zu keinen sinkenden $\mathrm{N}_{\min }$-Gehalten im Boden führte (LICKFETT, 1993). Bei der folgenden Darstellung des Bodennitrat-Zeitganges der verschiedenen Varianten werden deshalb die N-Düngestufen zusammengefaßt. 

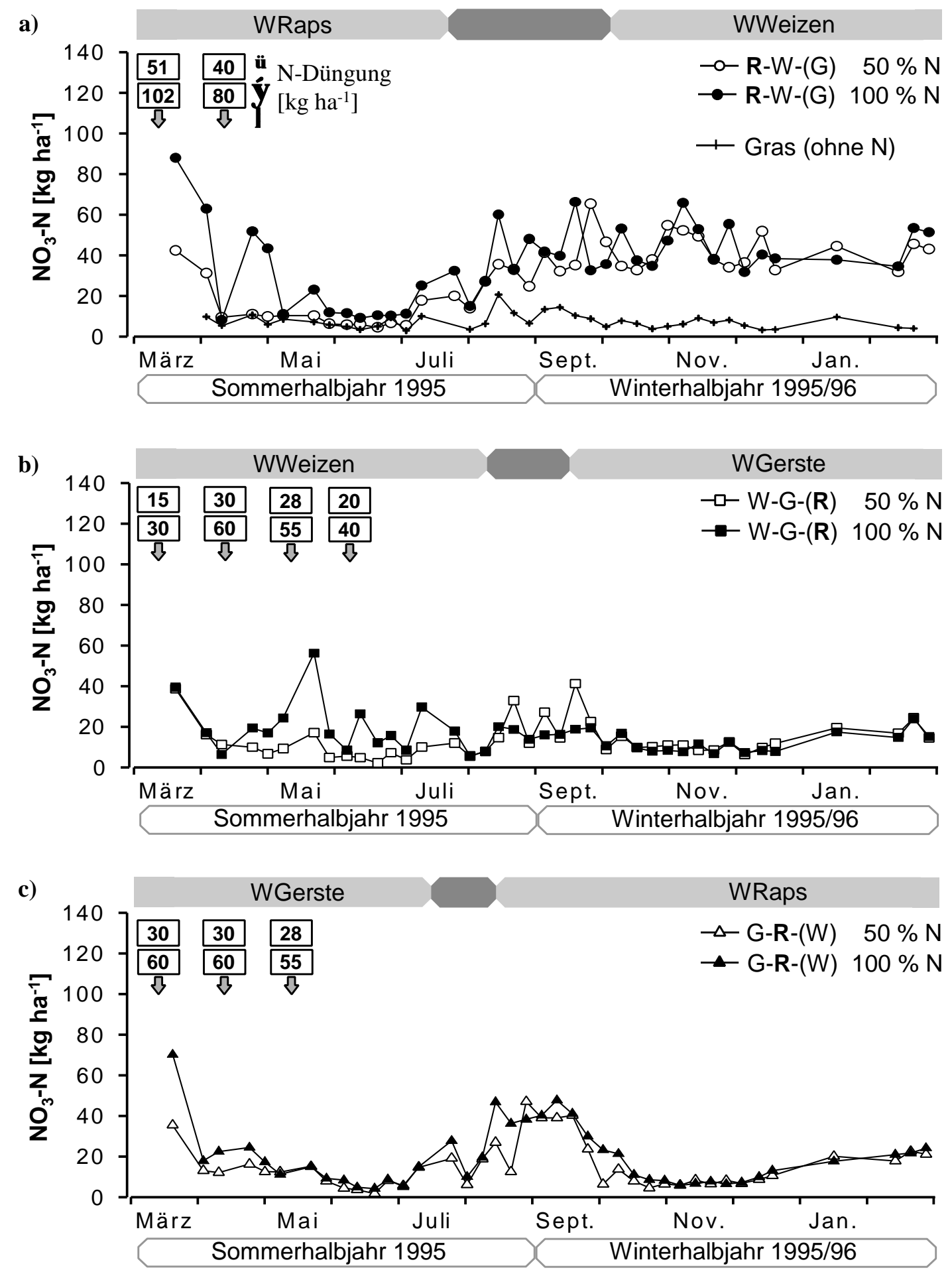

Abb. 3-8: Zeitlicher Verlauf der Nitratgehalte in 0-30 $\mathrm{cm}$ Bodentiefe in der gesamten Rotation einer Rapsfruchtfolge im Untersuchungszeitraum März 1995 bis Februar 1996. 


\section{5/96}

Unter WRaps (Abb. 3-8 (a)) lag im Sommerhalbjahr 1995 die Nitratkonzentration in 0-30 cm Bodentiefe mit $23 \mathrm{~kg} \mathrm{ha}^{-1}$ unter dem Durchschnitt, im Mai und Juni zeigten sich Nitratgehalte $<10 \mathrm{~kg} \mathrm{ha}^{-1}$. In der Zeit von Juli bis August stiegen die Nitratgehalte wieder auf Werte bis max. $60 \mathrm{~kg} \mathrm{ha}^{-1}$ an. In dem sich anschließenden Winterhalbjahr hielten sich die Bodennitratgehalte in $0-30 \mathrm{~cm}$ Tiefe im Mittel bei $43 \mathrm{~kg} \mathrm{ha}^{-1}$, im September wurden mit $66 \mathrm{~kg} \mathrm{ha}^{-1}$ maximale Nitratgehalte erreicht.

Beim WWeizen (Abb. 3-8 (b)) kam es im Sommerhalbjahr 1995 lediglich infolge der Frühjahrsdüngung zu Bodennitratgehalten von max. $60 \mathrm{~kg} \mathrm{ha}^{-1}$. Im Mittel lagen sie während dieser Zeit bei $18 \mathrm{~kg} \mathrm{ha}^{-1}$. Im Winterhalbjahr kam es unter der Folgefrucht WGerste zu keinem Nitratanstieg in den oberen $30 \mathrm{~cm}$ Boden, wie er nach WRaps zu verzeichnen war, die mittleren Gehalte lagen ähnlich denjenigen im Sommer unter WWeizen bei lediglich $14 \mathrm{~kg} \mathrm{ha}^{-1}$.

Unter WGerste (Abb. 3-8 $\quad$ (c)) zeigten sich im Sommerhalbjahr 1995 Bodennitratgehalte von durchschnittlich $18 \mathrm{~kg} \mathrm{ha}^{-1}$ in 0-30 cm Tiefe. Im Juni war kaum noch Nitrat nachzuweisen, nach der Ernte im Juli kam es jedoch zu einem deutlichen Anstieg bis auf Werte nahe $50 \mathrm{~kg} \mathrm{ha}^{-1}$. Nach dem Aufgang der Folgesaat WRaps im September (= Winterhalbjahr 1995/96) gingen die Nitratgehalte für den Rest des Winterhalbjahres auf Gehalte von z.T. deutlich unter $20 \mathrm{~kg} \mathrm{ha}^{-1}$ in $0-30 \mathrm{~cm}$ Bodentiefe zurück.

Auf der ungedüngten Grasfläche (Kontrolle) (Abb. 3-8 (a)) lagen die Nitratgehalte in den oberen $30 \mathrm{~cm}$ Boden sowohl im Sommerhalbjahr 1995 als auch im Winterhalbjahr 1995/96 im Mittel unter $10 \mathrm{~kg} \mathrm{~N} \mathrm{ha}^{-1}$, sie unterlagen außerdem keinen jahreszeitlich- oder bewirtschaftungsbedingten Schwankungen.

\section{6/97}

Unter WRaps (Abb. 3-9 (c)) zeigten sich im Sommerhalbjahr 1996 von März bis Anfang Mai infolge der N-Düngung Nitratgehalte von über $100 \mathrm{~kg} \mathrm{~N} \mathrm{ha}^{-1}$. Im gesamten Sommerhalbjahr lagen mittlere Nitratgehalte von $41 \mathrm{~kg} \mathrm{~N} \mathrm{ha}^{-1}$ vor. Die Gehalte ab Mai sanken auf konstante Werte $<20 \mathrm{~kg}^{\text {Nitrat }-\mathrm{N}} \mathrm{ha}^{-1}$. Unter der Folgefrucht WWeizen stiegen die Nitratkonzentrationen in diesem Winterhalbjahr nicht an und waren somit nicht so ausgeprägt wie diejenigen im Winterhalbjahr 1995/96 mit durchschnittlich 43 kg Nitrat-N ha ${ }^{-1}$. Sie lagen aber immer noch deutlich über den mittleren Nitratgehalten im Winterhalbjahr 1995/96 nach WWeizen oder WGerste. 

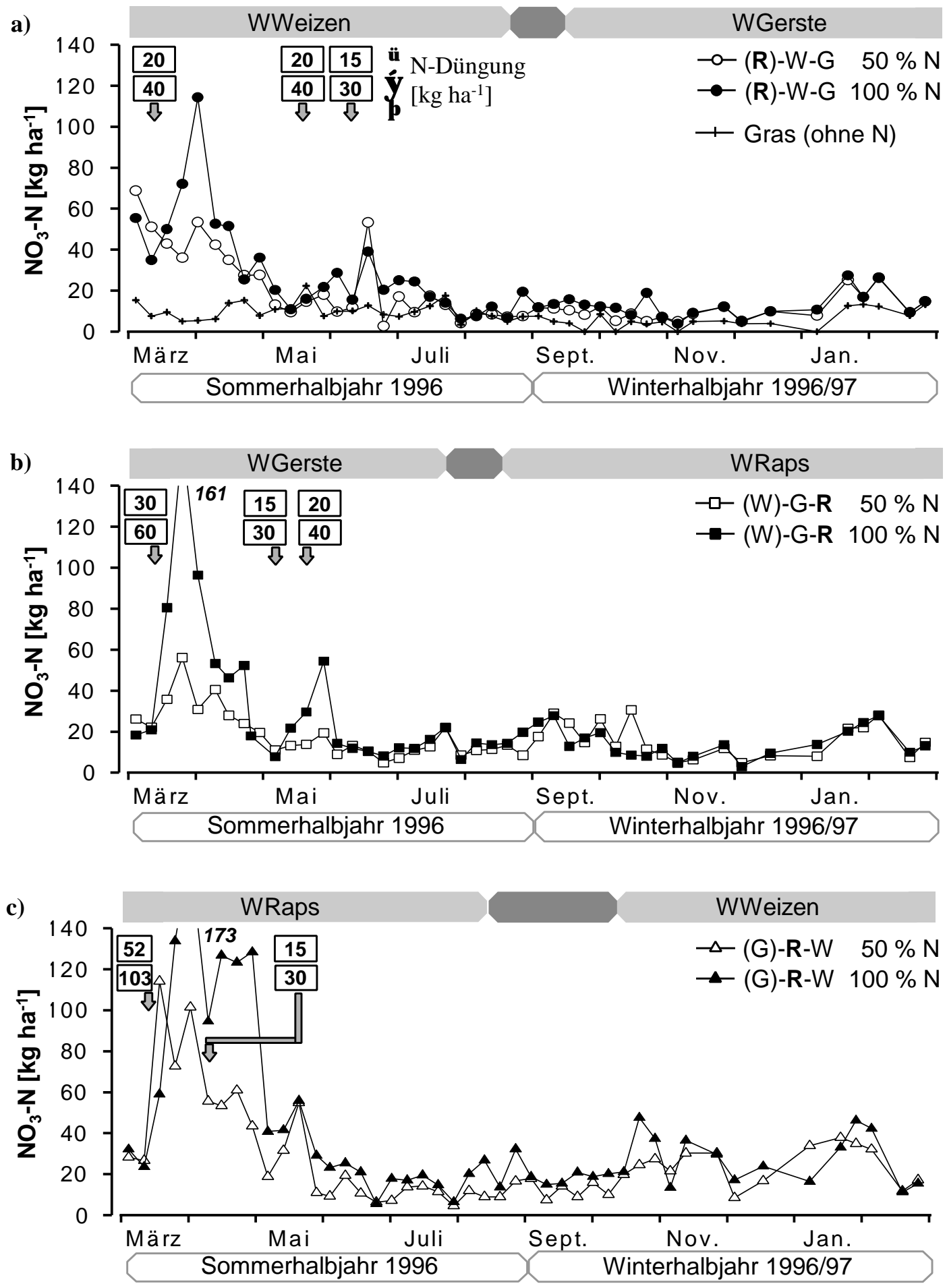

Abb. 3-9: Zeitlicher Verlauf der Nitratgehalte in 0-30 $\mathrm{cm}$ Bodentiefe in der gesamten Rotation einer Rapsfruchtfolge im Untersuchungszeitraum März 1996 bis Februar 1997. 
Beim WWeizen (Abb. 3-9 (a)) traten im Sommerhalbjahr 1996 vor allen Dingen im März/April in 0-30 cm Bodentiefe noch relativ hohe Nitratgehalte auf. Dieses ist zum einen offensichtlich auf die N-Gabe im März zurückzuführen, zum anderen auch auf hohe Nitratmengen im vorangegangenen Winterhalbjahr (ca. $60 \mathrm{~kg}^{\text {Nitrat}}-\mathrm{N} \mathrm{ha}{ }^{-1} \mathrm{im}$ Februar 1996). Unter der auf WWeizen folgenden WGerste wurden im Winterhalbjahr im Mittel lediglich Gehalte von $15 \mathrm{~kg}$ Nitrat-N ha ${ }^{-1}$ ermittelt.

Unter der WGerste (Abb. 3-9 (b)) wurden im Sommerhalbjahr 1996 mit durchschnittlich $25 \mathrm{~kg}$ Nitrat- $\mathrm{N}$ ha ${ }^{-1}$ höhere Nitratgehalte als im Vergleichszeitraum des Vorjahres nachgewiesen. Dieses dürfte, wie auch beim WRaps und WWeizen, auf das erst verspätet einsetzende Wachstum ab Mitte April (nach der ersten NGabe) und die damit verbundene verzögerte N-Aufnahme durch die Pflanzen zurückzuführen sein. Es zeigte sich weder nach der Stoppelbearbeitung noch beim darauffolgenden WRaps ein deutlicher Anstieg der Nitratgehalte. Im Winterhalbjahr 1996/97 wurden unter WRaps mittlere Gehalte in Höhe von $15 \mathrm{~kg}^{\text {Nitrat }-\mathrm{N}}$ ha $^{-1}$ in 0-30 cm Bodentiefe nachgewiesen.

Wie auch im Vorjahr lagen die Nitratgehalte auf der ungedüngten Grasfläche (Kontrolle) (Abb. 3-9 (a)) in den oberen $30 \mathrm{~cm}$ Boden im Mittel unter $10 \mathrm{~kg} \mathrm{~N} \mathrm{ha}^{-1}$, sie unterlagen außerdem keinen jahreszeitlich- oder bewirtschaftungsbedingten Schwankungen.

\subsubsection{Verlauf der $\mathrm{N}_{2} \mathrm{O}$-Emissionen}

\section{5/96}

Bei Gesamtbetrachtung beider N-Stufen beliefen sich die $\mathrm{N}_{2} \mathrm{O}-\mathrm{N}$-Emissionen aus der WRapsfläche (Abb. 3-10 (a)) im Sommerhalbjahr 1995 auf durchschnittlich 11,5 $\mu \mathrm{g}$ $\mathrm{m}^{-2} \mathrm{~h}^{-1}$. Im März und April wurden kurz nach den beiden N-Gaben kurzfristig leicht erhöhte Emissionsraten von maximal $43 \mu \mathrm{g} \mathrm{N} \mathrm{N}_{2} \mathrm{O}-\mathrm{N} \mathrm{m}^{-2} \mathrm{~h}^{-1}$ festgestellt. Erst im darauffolgenden Winterhalbjahr 1995/96 kam es unter der Folgefrucht WWeizen im Dezember zu einem deutlichen Anstieg der $\mathrm{N}_{2} \mathrm{O}$-Emissionen, die im Februar maximale Werte von fast $100 \mu \mathrm{g} \mathrm{N}_{2} \mathrm{O}-\mathrm{N} \mathrm{m}^{-2} \mathrm{~h}^{-1}$ erreichten. 

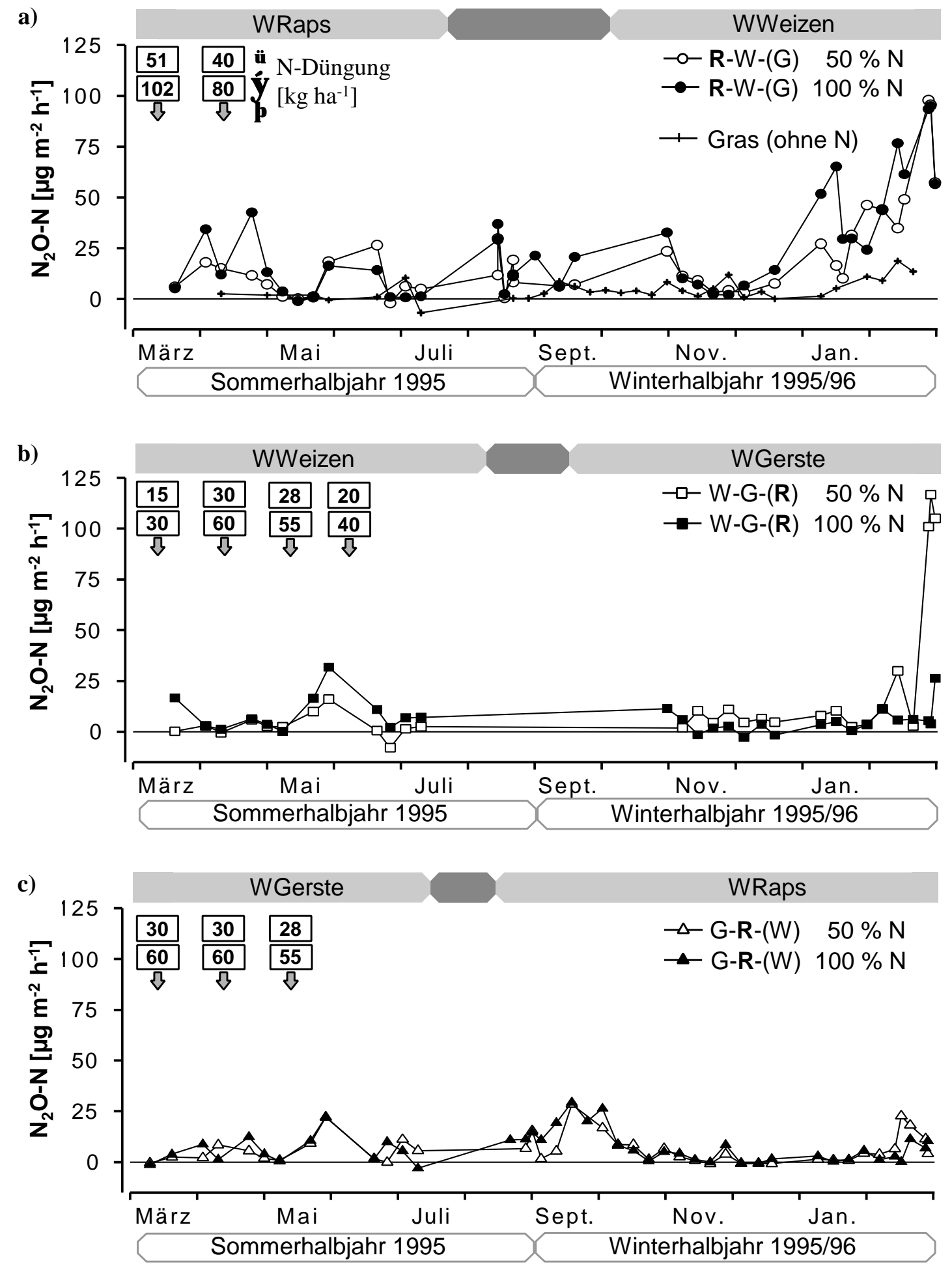

Abb. 3-10: Zeitlicher Verlauf der $\mathrm{N}_{2} \mathrm{O}-\mathrm{N}-$ Flüsse in der gesamten Rotation einer Rapsfruchtfolge im Untersuchungszeitraum März 1995 bis Februar 1996. 
Die WWeizenfläche (Abb. 3-10 (b)) zeigte in der Zeit von Mitte März bis Mitte Juli (Sommerhalbjahr 1995) mit durchschnittlichen Emissionen von $<10 \mu \mathrm{g} \mathrm{N}_{2} \mathrm{O}-\mathrm{N} \mathrm{m}^{-2} \mathrm{~h}^{-}$

${ }^{1}$ sehr geringe Flußraten. Lediglich Ende Mai stiegen sie parallel zu einer starken Wiederbefeuchtung des Bodens (Vgl. Abb. 3-6) auf Werte nahe $30 \mu \mathrm{g} \mathrm{N}_{2} \mathrm{O}-\mathrm{N} \mathrm{m}^{-2} \mathrm{~h}^{-1}$ an. Ein vergleichbarer Anstieg der Flußraten deutete sich zur gleichen Zeit sowohl im WRaps als auch in der WGerste an. In der Zeit von Mitte Juli bis Ende Oktober wurden auf dieser Fläche aus technischen Gründen keine Messungen durchgeführt. In den Monaten November 1995 bis Januar 1996 zeigten sich jedoch unter der Folgefrucht WGerste unverändert niedrige Emissionen. Im Februar kam es, wie schon auf der Vergleichsfläche unter WWeizen, zu einem starken, aber kurzfristigeren Anstieg der Flußraten auf Werte von $>100 \mu \mathrm{g} \mathrm{N}_{2} \mathrm{O}-\mathrm{N} \mathrm{m}^{-2} \mathrm{~h}^{-1}$.

Unter der WGerste (Abb. 3-10 (c)) wurden im Sommerhalbjahr 1995 sehr niedrige Emissionen von durchschnittlich $<10 \mu \mathrm{g} \mathrm{N}_{2} \mathrm{O}-\mathrm{N} \mathrm{m}^{-2} \mathrm{~h}^{-1}$ gemessen, auch unter der Folgefrucht WRaps erhöhte sich im Winterhalbjahr 1995/96 das Emissionsniveau nicht. Im gesamten Versuchsjahr waren auf dieser Fläche keine besonderen Emissionsereignisse festzustellen.

Die $\mathrm{N}_{2} \mathrm{O}-\mathrm{N}$-Emissionen des ungedüngten Graslandes (Abb. 3-10 (a)) waren über das gesamte Versuchsjahr sehr niedrig, im Mittel lagen sie unter $5 \mu \mathrm{g} \mathrm{N}_{2} \mathrm{O}-\mathrm{N} \mathrm{m} \mathrm{m}^{-2} \mathrm{~h}^{-1}$. Eine Saisonalität der Flußraten war hier nicht zu erkennen.

\section{$1996 / 97$}

Unter WRaps (Abb. 3-11 (c)) wurden, wie schon im Vergleichszeitraum des Vorjahres im Sommerhalbjahr 1996, mit durchschnittlich $9 \mu \mathrm{g} \mathrm{N}_{2} \mathrm{O}-\mathrm{N} \mathrm{m} \mathrm{m}^{-2} \mathrm{~h}^{-1}$ wiederholt sehr niedrige Emissionen ermittelt. Unmittelbar nach der Rapsernte stiegen die Emissionen deutlich an. Im darauffolgenden Winterhalbjahr 1996/97 zeigte sich erneut, daß nach WRaps unter der Folgefrucht WWeizen deutlich erhöhte $\mathrm{N}_{2} \mathrm{O}$-Emissionen auftreten können. Im Versuchsmittel wurden ca. $37 \mathrm{\mu g}$ $\mathrm{N}_{2} \mathrm{O}-\mathrm{N} \mathrm{m}^{-2} \mathrm{~h}^{-1}$ emittiert, im Januar 1997 wurden maximale Emissionsereignisse in Höhe von fast $400 \mu \mathrm{g} \mathrm{N} \mathrm{N}_{2} \mathrm{O}-\mathrm{N} \mathrm{m}^{-2} \mathrm{~h}^{-1}$ festgestellt.

Unter WWeizen (Abb. 3-11 (a)), der Folgefrucht nach WRaps, setzten sich im März 1996 die hohen $\mathrm{N}_{2} \mathrm{O}$-Emissionen des vorangegangenen Winterhalbjahres mit maximalen Werten von $175 \mu \mathrm{g} \mathrm{N}_{2} \mathrm{O}-\mathrm{N} \mathrm{m}^{-2} \mathrm{~h}^{-1}$ (in N $50 \%$ ) fort. Ab April sanken sie jedoch auf ein niedriges Niveau, so daß es im Sommerhalbjahr 1996 unter WWeizen zu mittleren Emissionen $<20 \mu \mathrm{g} \mathrm{N}_{2} \mathrm{O}-\mathrm{N} \mathrm{m}^{-2} \mathrm{~h}^{-1} \mathrm{kam}$. Bereinigt um die extrem hohen Märzemissionen wies das Sommerhalbjahr mittlere Emissionen von nur $14 \mu \mathrm{g} \mathrm{N} \mathrm{N}_{2} \mathrm{O}-\mathrm{N}$ $\mathrm{m}^{-2} \mathrm{~h}^{-1}$ auf. Damit lagen 1996 die mittleren Sommeremissionen unter Winterweizen auf gleichem Niveau mit denjenigen des WRapses. Im folgenden Winterhalbjahr 

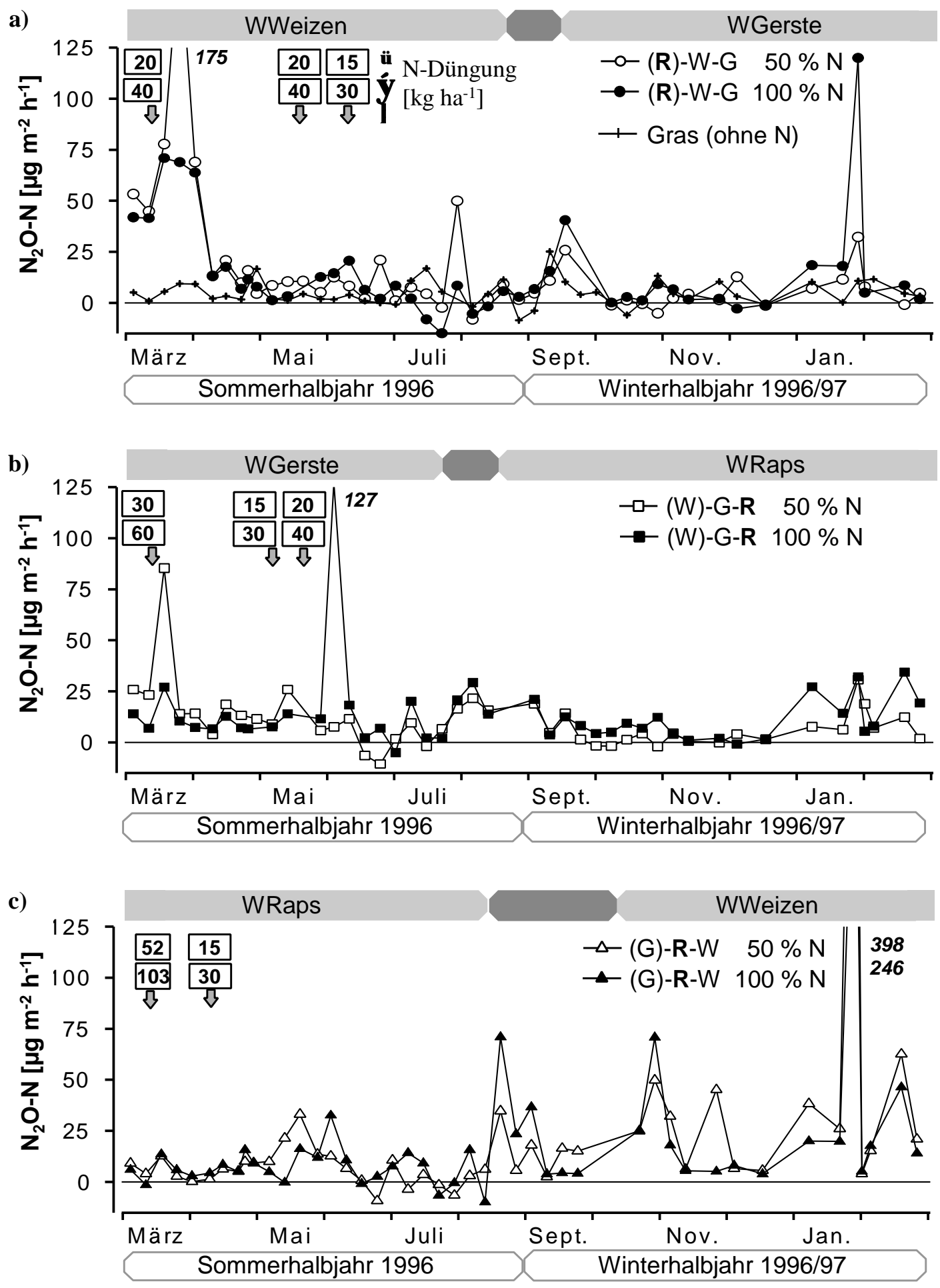

Abb. 3-11: Zeitlicher Verlauf der $\mathrm{N}_{2} \mathrm{O}-\mathrm{N}-$ Flüsse in der gesamten Rotation einer Rapsfruchtfolge im Untersuchungszeitraum März 1996 bis Februar 1997. 
1996/97 traten unter der Folgefrucht WGerste mit $10 \mu \mathrm{g} \mathrm{N}_{2} \mathrm{O}-\mathrm{N} \mathrm{m}^{-2} \mathrm{~h}^{-1}$ ähnlich niedrige mittlere Emissionen wie im Vergleichszeitraum des Vorjahres auf. Wie auch schon unter WWeizen beobachtet, zeigte sich auch unter WGerste Ende Januar ein maximaler Emissionspeak. Dieser fiel mit dem Auftauen des Bodens nach fast 6 Wochen Dauerfrost zusammen (Vgl. Abb. 3-5).

In der WGerste (Abb. 3-11 (b)) wurden im Sommerhalbjahr 1996 durchschnittlich $15 \mathrm{\mu g} \mathrm{N}_{2} \mathrm{O}-\mathrm{N} \mathrm{m}^{-2} \mathrm{~h}^{-1}$ emittiert. Im März wurde zwar infolge der ersten N-Düngung ein Emissionspeak verzeichnet, im Gegensatz zu den $\mathrm{N}_{2} \mathrm{O}$-Emissionen im WWeizen war das Emissionsniveau in diesem Monat jedoch schwächer ausgeprägt. Wie auch schon im Vorjahr beobachtet, kam es im folgenden Winterhalbjahr 1996/97 unter WRaps zu sehr niedrigen $\mathrm{N}_{2} \mathrm{O}-\mathrm{N}-$ Emissionen von $<10 \mu \mathrm{g} \mathrm{N}_{2} \mathrm{O}-\mathrm{N} \mathrm{m}^{-2} \mathrm{~h}^{-1}$.

Auch im Versuchsjahr 1996/97 zeigte das ungedüngte Grasland (Abb. 3-11 (a)) mit Werten $<5 \mu \mathrm{g} \mathrm{N} \mathrm{N}_{2} \mathrm{O}-\mathrm{N} \mathrm{m}^{-2} \mathrm{~h}^{-1}$ sehr niedrige Emissionen; eine Saisonalität der Flußraten war auch in diesem Versuchsjahr nicht zu erkennen.

Es ist zu erkennen, daß das $\mathrm{N}_{2} \mathrm{O}$-Emissionsniveau auf dem Standort Reinshof relativ gering war. Infolge von N-Düngemaßnahmen deuteten sich lediglich leichte Emissionserhöhungen an, eine Ausnahme stellten diesbezüglich nur die hohen Emissionspeaks nach der ersten N-Applikation im März 1996 dar. Eine Reduzierung der N-Düngung um $50 \%$ führte in beiden Versuchsjahren weder in den Sommerhalbjahren noch in den Winterhalbjahren zu Veränderungen der $\mathrm{N}_{2} \mathrm{O}$ Emissionen. Innerhalb der WRaps-Fruchtfolge wurden die höchsten N-Emissionen im Herbst/Winter nach WRaps gemessen. Deutlich erhöhte Emissionen traten während der Frost-Tau Perioden auf.

\subsubsection{Einflüsse der Bodenparameter auf die $\mathrm{N}_{2} \mathrm{O}$-Emissionen der verschiedenen Fruchtarten}

Die Ergebnisse der Untersuchungen zeigen deutlich, daß in der Fruchtfolge R-W-G die $\mathrm{N}_{2} \mathrm{O}$-Emissionen maßgeblich durch die Vorfruchtwirkung des Rapses beeinflußt werden. Der Einfluß der Fruchtart auf die $\mathrm{N}_{2} \mathrm{O}$-Freisetzung geht also über den Erntezeitpunkt hinaus. Um die Einflüsse der Bodenparameter auf die $\mathrm{N}_{2} \mathrm{O}$ Emissionen verschiedener Fruchtarten beschreiben zu können, wurde deshalb, wie in Kapitel 3.2.4 bereits beschrieben, das Winterhalbjahr mit der Folgefrucht jeweils der voranstehenden Vorfrucht zugeordnet. Um für die einzelnen Fruchtarten die Einflüsse von Bodenfeuchte, Bodentemperatur und Nitratgehalt auf die $\mathrm{N}_{2} \mathrm{O}$ Emissionen quantifizieren zu können, wurden Korrelationen zwischen diesen 
Parametern und den $\mathrm{N}_{2} \mathrm{O}$-Flußraten berechnet, wobei nicht zwischen den jeweiligen $\mathrm{N}$-Stufen differenziert wurde.

Unter WRaps (Tab. 3-6) zeigten sich in den Sommerhalbjahren 1995 und 1996 keine signifikanten Zusammenhänge zwischen den Emissionsraten und den parallel bestimmten Bodenparametern. Maximale Sommeremissionsereignisse, wie sie z.B. Mitte August 1996 nach der Rapsernte auftraten, waren nur von kurzer Dauer. In den folgenden Winterhalbjahren begünstigten hohe Bodenwassergehalte (Abb. 3-6 und 3-7) die $\mathrm{N}_{2} \mathrm{O}$-Freisetzung. Hier konnten $31 \%$ (Winterhalbj. 95/96) bzw. $24 \%$ (Winterhalbj. 96/97) der Variabilität der Emissionsraten über den WFPS erklärt werden.

Tab. 3-6: Korrelationskoeffizienten zwischen den möglichen Einflußfaktoren (WFPS, Bodentemperatur, Bodennitratgehalte) und den $\mathrm{N}_{2} \mathrm{O}-\mathrm{N}$-Flußraten sowie die Bestimmtheits-maße der einfachen bzw., wo möglich, der schrittweisen multiplen Regression.

\begin{tabular}{|c|c|c|c|c|c|c|c|c|c|c|c|c|}
\hline \multirow[b]{2}{*}{$\begin{array}{c}\text { Frucht- } \\
\text { art }\end{array}$} & \multicolumn{3}{|c|}{ Sommerhalbj. 1995} & \multicolumn{3}{|c|}{ Sommerhalbj. 1996} & \multicolumn{3}{|c|}{ Winterhalbj. 1995/96 } & \multicolumn{3}{|c|}{ Winterhalbj. 1996/97 } \\
\hline & $\begin{array}{l}\text { Einfuß- } \\
\text { faktor }\end{array}$ & $\mathbf{r}$ & $r^{2}$ & $\begin{array}{l}\text { Einfuß- } \\
\text { faktor }\end{array}$ & $\mathbf{r}$ & $r^{2}$ & $\begin{array}{l}\text { Einfuß- } \\
\text { faktor }\end{array}$ & $\mathbf{r}$ & $r^{2}$ & $\begin{array}{l}\text { Einfuß- } \\
\text { faktor }\end{array}$ & $\mathbf{r}$ & $r^{2}$ \\
\hline \multirow[t]{2}{*}{ WRa } & n.s. & & & n.s. & & & $\mathbf{W}^{\star \star}$ & 0,56 & 0,31 & $\mathbf{W}^{* \star}$ & 0,49 & 0,24 \\
\hline & & & & & & & & & & $\log N^{*}$ & 0,39 & \\
\hline \multirow[t]{3}{*}{ ww } & n.s. & & & $\mathbf{W}^{* \star *}$ & 0,55 & 0,30 & n.s. & & & n.s. & & \\
\hline & & & & $\mathrm{T}^{\star * *}$ & $-0,54$ & & & & & & & \\
\hline & & & & $\log N^{* * *}$ & 0,40 & & & & & & & \\
\hline WG & $T^{*}$ & 0,41 & 0,17 & $W^{*}$ & 0,31 & 0,10 & $\log N^{* *}$ & 0,51 & 0,26 & n.s. & & \\
\hline
\end{tabular}

Die Messungen des Winterhalbjahres wurden jeweils der vorangestellten Hauptfrucht zugeordnet.

$\mathrm{r}=$ Korrelationskoeffizient der einfachen linearen Korrelation;

$r^{2}=$ Bestimmtheitsmaß der in die multiple Regression mit eingeflossenen Einflußfaktoren;

Einflußfaktor (fett) $\Rightarrow$ in multiple Regression mit eingeflossen;

n.s. = Einflußfaktoren nicht signifikant;

$\mathrm{W}=$ WFPS; $\mathrm{T}=$ Bodentemperatur; $\mathrm{N}=$ Bodennitrat;

$* \mathrm{p}<0,05 ; * * \mathrm{p}<0,01 ; * * * \mathrm{p}<0,001$

Beim WWeizen (Tab. 3-6) konnten während des Sommerhalbjahres 1995, berechnet über beide N-Stufen, keine signifikanten Korrelationen zwischen der Emissionshöhe und den Steuergrößen gefunden werden. Im Sommerhalbjahr 1996 waren die Emissionen hochsignifikant positiv mit dem WFPS und dem Nitratgehalt 
des Bodens sowie aufgrund der Frostperiode im Frühjahr negativ mit der Bodentemperatur korreliert. Da die Steuergrößen untereinander jedoch eng korreliert waren, ging als einziger Faktor das wassergefüllte Porenvolumen mit einem Bestimmtheitsmaß von $30 \%$ in die schrittweise multiple Regression ein. Zwischen den Steuergrößen und den Lachgasemissionen bestanden bei dieser Frucht für die Winterhalbjahre keine signifikanten Korrelationen.

Unter WGerste (Tab. 3-6) zeigten sich im Sommer wie auch im Winter nur sehr schwache Zusammenhänge zwischen den Emissionsraten und den gemessenen Steuergrößen. Das höchste Bestimmtheitsmaß $\left(r^{2}=0,26\right)$ wurde, berechnet über beide N-Stufen, im Winterhalbjahr 1995/96 mit dem Nitratgehalt erreicht.

\subsubsection{Kumulierte $\mathrm{N}_{2} \mathrm{O}$-Emissionen}

Um die gemessenen Emissionen beim Anbau von WRaps, WWeizen und WGerste sowie die der Grasfläche miteinander vergleichen zu können und evtl. fruchtartspezifische Unterschiede herauszuarbeiten, wurden die jährlichen Gasflüsse stufenweise kumulativ berechnet. Dabei wurde davon ausgegangen, daß die Flußraten zwischen zwei Meßterminen konstant waren.

In Tabelle 3-7 sind die kumulierten $\mathrm{N}_{2} \mathrm{O}$-Emissionen für die verschiedenen Fruchtarten in den Sommer- und Winterhalbjahren sowie für das jeweilige Gesamtjahr 1995/96 und 1996/97 (März - Februar) dargestellt.

Auf der WRapsfläche trat mit 1,8 $\mathrm{kg} \mathrm{N}_{2} \mathrm{O}-\mathrm{N} \mathrm{ha}^{-1}$ im Jahr 1996/97 in der stickstoffreduzierten Variante die höchste Jahresemission des gesamten Versuchszeitraumes auf (Tab. 3-7). Im Vergleich zu den anderen Flächen wurde in beiden Jahren auf den WRaps-Flächen das meiste $\mathrm{N}_{2} \mathrm{O}$ emittiert $(1,25$ bis $1,8 \mathrm{~kg}$ $\mathrm{N}_{2} \mathrm{O}-\mathrm{N}$ ha ${ }^{-1}$ ). Die deutlich erhöhte Emission aus dem WRaps hatte ihren Ursprung in den Winteremissionen, die im Mittel $71 \%$ der Gesamtjahresemissionen der Rapsfläche ausmachten. Der WRaps war die einzige Fruchtart, die im Winterhalbjahr signifikant höhere $\mathrm{N}_{2} \mathrm{O}$-Emissionen aufwies als die ungedüngte Rasenfläche. 
Tab. 3-7: Kumulierte $\mathrm{N}_{2} \mathrm{O}-\mathrm{N}-E m i s s i o n e n$ in den Sommer- und Winterhalbjahren sowie für das Gesamtjahr 1995/96 und 1996/97 (März - Februar).

\begin{tabular}{|c|c|c|c|c|c|c|c|c|c|c|c|}
\hline \multirow[t]{2}{*}{$\begin{array}{l}\text { Frucht- } \\
\text { art }\end{array}$} & \multicolumn{2}{|c|}{ N-Düngung } & \multicolumn{3}{|c|}{$\begin{array}{l}\text { Emissionen } \\
\text { Sommerhalbjahr } \\
\text { [kg N } \mathrm{N}_{2} \mathrm{O}-\mathrm{N} \mathrm{ha}^{-1} \text { ] }\end{array}$} & \multicolumn{3}{|c|}{$\begin{array}{c}\text { Emissionen } \\
\text { Winterhalbjahr } \\
{\left[\mathrm{kg} \mathrm{N}_{2} \mathrm{O}-\mathrm{N} \mathrm{ha}^{-1}\right]}\end{array}$} & \multicolumn{3}{|c|}{$\begin{array}{l}\text { Emissionen } \\
\text { Gesamtjahr } \\
{\left[\mathrm{kg} \mathrm{N}_{2} \mathrm{O}-\mathrm{N} \mathrm{ha}^{-1}\right]}\end{array}$} \\
\hline & 1995 & 1996 & 1995 & 1996 & Mittel & $1995 / 96$ & $1996 / 97$ & Mittel & $1995 / 96$ & $1996 / 97$ & Mittel \\
\hline WRa & 182 & 133 & $\begin{array}{c}0,42^{a b} \\
(34) \\
0,54^{a} \\
(31)\end{array}$ & $\begin{array}{c}0,34^{C} \\
(19) \\
0,45^{c} \\
(31)\end{array}$ & 0,50 & $\begin{array}{c}0,83^{a b} \\
(66) \\
1,18^{a} \\
(69)\end{array}$ & $\begin{array}{c}1,47^{a} \\
(81) \\
1,01^{a} \\
(69)\end{array}$ & 1,10 & 1,71 & 1,46 & 1,59 \\
\hline WW & 185 & 110 & $\begin{array}{c}\mathbf{0 , 1 7 ^ { c d }} \\
(29) \\
0,39^{a b} \\
(60)\end{array}$ & $\begin{array}{c}1,04^{a} \\
(79) \\
0,68^{b c} \\
(57)\end{array}$ & 0,54 & $\begin{array}{c}0,42^{b c} \\
(71) \\
0,26^{c} \\
(40)\end{array}$ & $\begin{array}{c}0,27^{b} \\
(21) \\
0,51^{b} \\
(43)\end{array}$ & 0,35 & 0,59 & 1,31 & 0,92 \\
\hline WG & 175 & 160 & $\begin{array}{c}0,26^{b c} \\
(49) \\
0,27^{b c} \\
(47)\end{array}$ & $\begin{array}{c}0,67^{b c} \\
(73) \\
0,70^{b c} \\
(59)\end{array}$ & 0,49 & $\begin{array}{c}0,27^{C} \\
(51) \\
0,30^{C} \\
(53)\end{array}$ & $\begin{array}{c}0,25^{b} \\
(27) \\
0,48^{b} \\
(41)\end{array}$ & 0,39 & 0,57 & 1,19 & 0,72 \\
\hline Gras & 0 & 0 & $\begin{array}{r}0,02^{d} \\
(8)\end{array}$ & $\begin{array}{r}0,18^{C} \\
(43)\end{array}$ & 0,10 & $\begin{array}{r}0,24^{C} \\
(92)\end{array}$ & $\begin{array}{c}0,24^{b} \\
(57)\end{array}$ & 0,24 & 0,26 & 0,42 & 0,34 \\
\hline
\end{tabular}

Die Messungen des Winterhalbjahres wurden jeweils der vorangestellten Hauptfrucht zugeordnet.

( ): $\quad$ prozentuale Anteile der Sommer- bzw. Winteremissionen am Gesamtjahr.

Indexbuchstaben: kennzeichnen signifikante Unterschiede $(\mathrm{p}<0,05)$ zwischen den Fruchtarten innerhalb des jeweiligen Halbjahres.

Die $\mathrm{N}_{2} \mathrm{O}$-Freisetzung aus den Getreideflächen variierte von $0,59-1,31 \mathrm{~kg} \mathrm{~N}_{2} \mathrm{O}-\mathrm{N}$ ha $^{-1}$ bei Weizen und von $0,53-1,19 \mathrm{~kg} \mathrm{~N}_{2} \mathrm{O}-\mathrm{N} \mathrm{ha}^{-1}$ bei Gerste. Der relative Anteil der Winteremissionen an den Gesamtjahresemissionen war sehr unterschiedlich.

Die niedrigste $\mathrm{N}_{2} \mathrm{O}$ - Jahresemission zeigte sich mit 0,26 kg $\mathrm{N}_{2} \mathrm{O}-\mathrm{N}$ ha ${ }^{-1}$ auf der Grasfläche (1995/96).

Bei einer Verminderung der N-Düngung um $50 \%$ kam es auf diesem Versuchsstandort in keiner Fruchtart zu einem signifikanten Rückgang der $\mathrm{N}_{2} \mathrm{O}$ - 
Emissionen $(p<0,05)$. Lediglich beim WWeizen zeigte sich im Sommer 1995 ein schwach signifikanter Rückgang.

Zusammenfassend kann festgestellt werden, daß im Versuchsmittel über die beiden Anbaujahre 1995/96 und 1996/97 (Abb. 3-12), berechnet über beide N-Stufen, auf dem Versuchsstandort beim Anbau von WRaps 1,6 $\mathrm{kg} \mathrm{N}_{2} \mathrm{O}-\mathrm{N}^{-1} \mathrm{ha}^{-1} \mathrm{a}^{-1}$ emittiert wurden. Im Vergleich dazu betrug die mittlere Jahresemission aus WWeizen 0,9 und die aus WGerste lediglich $0,8 \mathrm{~kg} \mathrm{~N}_{2} \mathrm{O}-\mathrm{N} \mathrm{ha}^{-1} \mathrm{a}^{-1}$. Die Abbildung zeigt die dominierende Bedeutung der $\mathrm{N}_{2} \mathrm{O}$-Emissionen im Winterhalbjahr nach der WRapsernte an der mit dieser Frucht verbundenen $\mathrm{N}_{2} \mathrm{O}$-Jahresemission. Im Winterhalbjahr (September - Februar) wurde hier ebensoviel $\mathrm{N}_{2} \mathrm{O}$ emittiert wie im Mittel der Fruchtfolge in einem ganzen Bilanzierungsjahr. Vom Grasland wurden im Versuchsmittel lediglich $0,34 \mathrm{~kg} \mathrm{~N}_{2} \mathrm{O}-\mathrm{N} \mathrm{ha}^{-1} \mathrm{a}^{-1}$ emittiert.

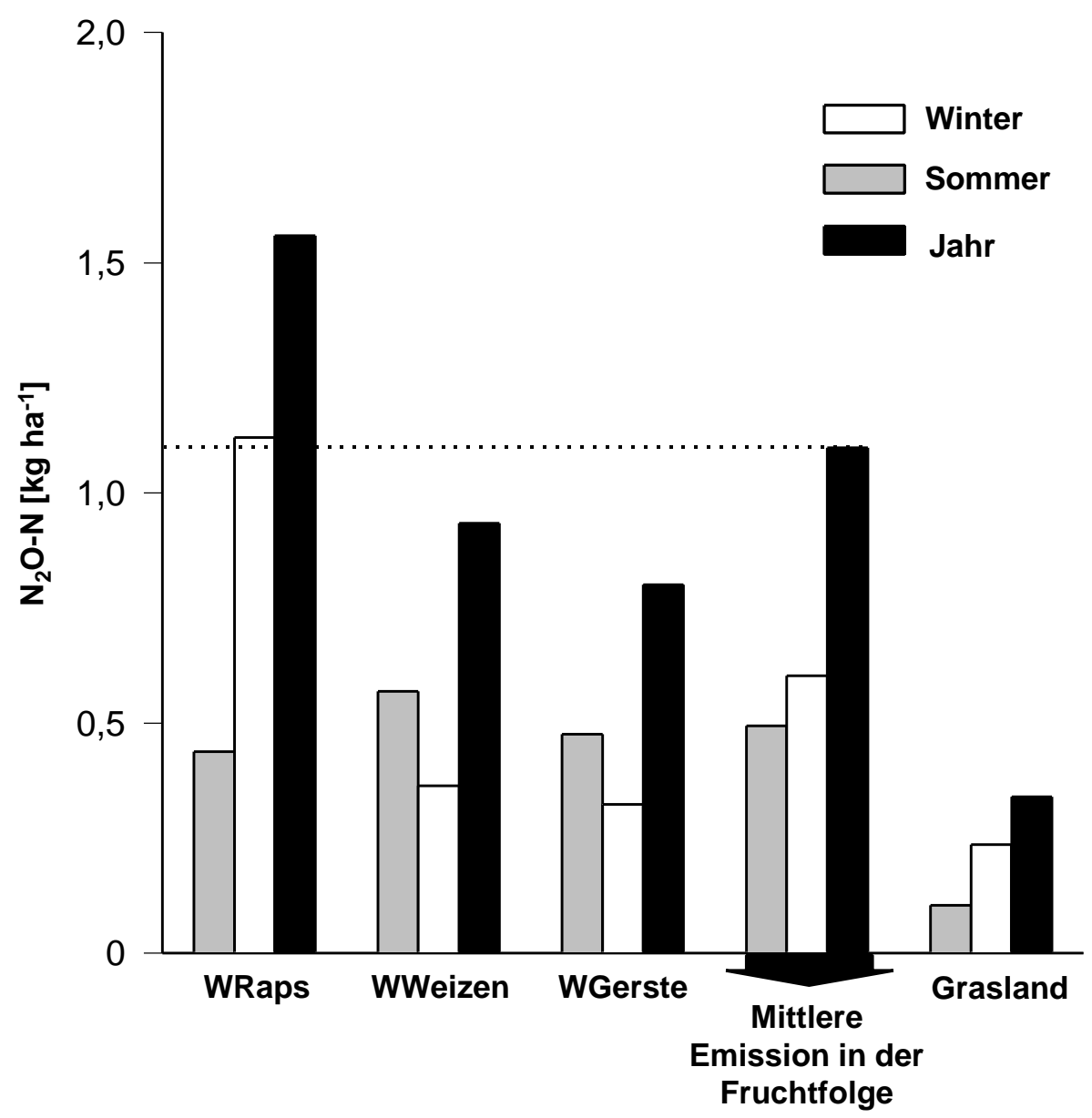

Abb. 3-12: Fruchtartspezifische $\mathrm{N}_{2} \mathrm{O}-\mathrm{N}-$ Gesamtemissionen auf Basis halbjährlich kumulierter $\mathrm{N}_{2} \mathrm{O}-\mathrm{N}$-Emissionen (Versuchsmittel) über beide $\mathrm{N}$-Stufen. Das Winterhalbjahr wurde jeweils der vorangestellten Hauptfrucht zugeordnet. 


\subsubsection{Verlauf der $\mathrm{CH}_{4}$-Flüsse}

Während des gesamten Untersuchungszeitraumes wies der Versuchsstandort fast ausschließlich negative Methanflüsse auf (Abb. 3-13 und Abb. 3-14). Das bedeutet, daß der Versuchsstandort eine Senke für atmosphärisches Methan darstellte. Fruchtartspezifische Spurengasflüsse, wie sie beim $\mathrm{N}_{2} \mathrm{O}$ beobachtet wurden, konnten bei den Methanflüssen nicht festgestellt werden.

Eine Reduktion der Stickstoffdüngung um $50 \%$ führte in keiner Variante zu einer eindeutigen Veränderung der Methanflüsse. In allen Versuchsvarianten war jedoch in beiden Versuchsjahren eine deutliche Saisonalität der Methanflüsse zu erkennen. Die Sommerhalbjahre zeichneten sich dabei zu Zeiten niedriger Wassergehalte des Bodens (vgl. Abb. 3-6 und Abb. 3-7) durch relativ hohe Methanaufnahmeraten aus. Im Versuchsmittel lagen die Flußraten in den Sommerhalbjahren bei ca. $-8 \mu \mathrm{g} \mathrm{m}^{-2} \mathrm{~h}^{-1}$ $\mathrm{CH}_{4}$-C. Im Sommerhalbjahr 1995 wurden maximale Methanaufnahmeraten von 22 $\mu \mathrm{g} \mathrm{m}^{-2} \mathrm{~h}^{-1}$ (Abb. 3-13 (a)), im Sommerhalbjahr 1996 sogar von $28 \mu \mathrm{g} \mathrm{m}^{-2} \mathrm{~h}^{-1}$ ermittelt (Abb. 3-14 (a) und c)). In den Winterhalbjahren 1995/96 wie auch 1996/97 lagen die mittleren Flußraten hingegen bei nur $-3 \mu \mathrm{g} \mathrm{m}^{-2} \mathrm{~h}^{-1} \mathrm{CH}_{4}-\mathrm{C}$. Parallel zu den noch relativ niedrigen Bodenwassergehalten im September wurden auch in den Winterhalbjahren noch maximale Methanaufnahmeraten von $>10 \mu \mathrm{g} \mathrm{m}^{-2} \mathrm{~h}^{-1}$ erreicht, mit zunehmenden Bodenwassergehalten sanken sie jedoch gegen Null.

Die Graslandfläche (Abb. 3-13 (a) und 3-14 (a)) wies zwar einen den Fruchtfolgevarianten vergleichbaren saisonalen Verlauf der Methanflüsse auf, jedoch auf einem besonders in den Sommerhalbjahren niedrigeren Niveau.

\subsubsection{Einflüsse der Bodenparameter auf die $\mathrm{CH}_{4}$-Flüsse}

In allen Fruchtarten wurde sowohl in den Sommer- als auch in den Winterhalbjahren eine negative Korrelation (Tab. 3-8) zwischen dem WFPS und der Methanoxidation gefunden. Im Mittel konnten rund $40 \%$ der zeitlichen Variation der $\mathrm{CH}_{4}$-Aufnahme über die Dynamik des WFPS erklärt werden. Die Temperaturen in 2,5 cm Bodentiefe korrelierten über den gesamten Versuchszeitraum in nahezu allen Varianten positiv mit der $\mathrm{CH}_{4}$-Oxidation (Versuchsmittel: $r=0,59$ ). Da die Steuergrößen WFPS und Bodentemperatur nicht unabhängig voneinander waren, ging jeweils nur das WFPS in die schrittweise multiple Regression ein. 

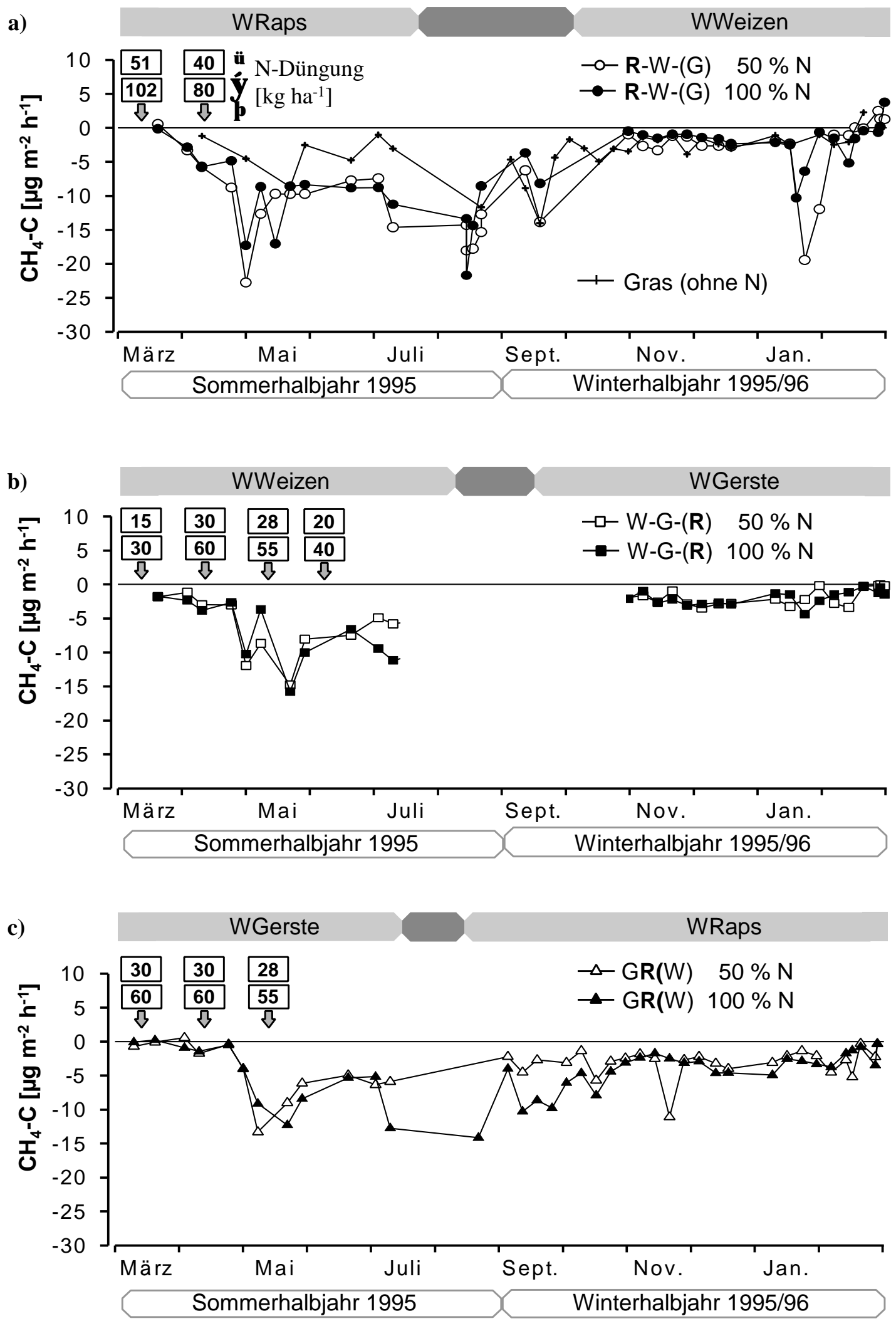

Abb. 3-13: Zeitlicher Verlauf der $\mathrm{CH}_{4}$-C-Flüsse in der gesamten Rotation einer Rapsfruchtfolge im Untersuchungszeitraum März 1995 bis Februar 1996. 

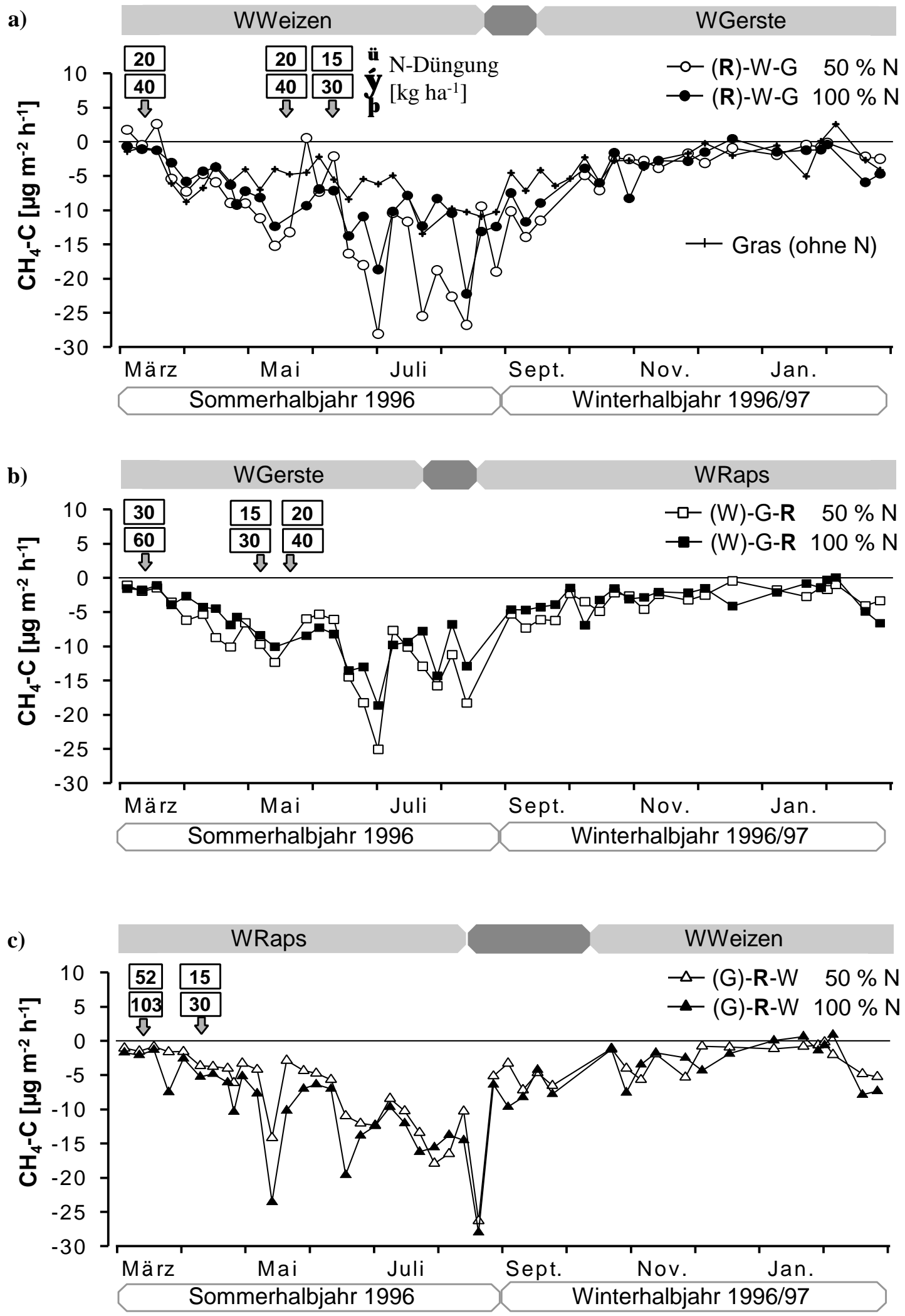

Abb. 3-14: Zeitlicher Verlauf der $\mathrm{CH}_{4}$-C-Flüsse in der gesamten Rotation einer Rapsfruchtfolge im Untersuchungszeitraum März 1996 bis Februar 1997. 
Nur im Sommer 1996 korrelierte in allen Varianten der Nitratgehalt des Bodens negativ mit der $\mathrm{CH}_{4}$-Oxidation (Tab. 3-8). Er erbrachte ausschließlich in der WGerste gemeinsam mit dem WFPS ein Bestimmtheitsmaß von $56 \%$. Im WRaps und auch im WWeizen wurden dagegen die Nitratgehalte aufgrund starker Korrelationen der Steuergrößen untereinander nicht in die schrittweise multiple Regression aufgenommen. In Abb. 3-14 wurde aber deutlich, daß es im Sommerhalbjahr 1996 nach den Ammoniumnitratdüngungen in den Monaten Mai und Juni zu einer Verringerung der $\mathrm{CH}_{4}$-Oxidationsraten kam. Wie in Kapitel 2.2.2 ausgeführt, ist eine mögliche Ursache hierfür die geringe Substratspezifität der Methanoxigenase, die sowohl Methan als auch Ammonium oxidieren kann.

Tab. 3-8: Korrelationskoeffizienten zwischen den möglichen Einflußfaktoren (WFPS, Bodentemperatur, Bodennitratgehalte) und den $\mathrm{CH}_{4}$-C-Flußraten sowie die Bestimmtheits-maße der einfachen bzw., wo möglich, der schrittweisen multiplen Regression.

\begin{tabular}{|c|c|c|c|c|c|c|c|c|c|c|c|c|}
\hline \multirow[b]{2}{*}{$\begin{array}{c}\text { Frucht- } \\
\text { art }\end{array}$} & \multicolumn{3}{|c|}{ Sommerhalbj. 1995} & \multicolumn{3}{|c|}{ Sommerhalbj. 1996} & \multicolumn{3}{|c|}{ Winterhalbj. 1995/96 } & \multicolumn{3}{|c|}{ Winterhalbj. 1996/97 } \\
\hline & $\begin{array}{l}\text { Einfuß- } \\
\text { faktor }\end{array}$ & $r$ & $r^{2}$ & $\begin{array}{l}\text { Einfuß- } \\
\text { faktor }\end{array}$ & $\mathbf{r}$ & $r^{2}$ & $\begin{array}{l}\text { Einfuß- } \\
\text { faktor }\end{array}$ & $\mathbf{r}$ & $r^{2}$ & $\begin{array}{l}\text { Einfuß } \\
\text { faktor }\end{array}$ & $r$ & $r^{2}$ \\
\hline WRa & $\begin{array}{c}\log W^{\star \star \star} \\
T^{\star \star}\end{array}$ & $\begin{array}{r}-0,62 \\
0,44\end{array}$ & 0,39 & $\begin{array}{c}\mathbf{W}^{\star \star \star} \\
\mathrm{T}^{\star \star \star} \\
\log \mathrm{N}^{\star \star \star *}\end{array}$ & $\begin{array}{l}-0,59 \\
0,61 \\
-0,56\end{array}$ & 0,36 & $\mathbf{W}^{*}$ & $-0,46$ & 0,21 & $\begin{array}{l}\mathbf{W}^{\star \star \star} \\
T^{\star \star \star} \\
\log N\end{array}$ & $\begin{array}{r}-0,69 \\
0,63 \\
-0,44\end{array}$ & 0,47 \\
\hline wW & $\mathbf{W}^{*}$ & $-0,46$ & 0,21 & $\begin{array}{c}\mathbf{W}^{\star \star \star} \\
\mathrm{T}^{\star \star \star} \\
\log \mathrm{N}^{\star \star \star}\end{array}$ & $\begin{array}{r}-0,69 \\
0,59 \\
-0,57\end{array}$ & 0,48 & $\begin{array}{c}\mathbf{W}^{\star \star} \\
\mathrm{N}^{\star}\end{array}$ & $\begin{array}{l}-0,55 \\
-0,45\end{array}$ & 0,31 & $\begin{array}{l}\mathbf{W}^{\star \star \star} \\
T^{\star \star \star}\end{array}$ & $\begin{array}{l}-0,69 \\
0,76\end{array}$ & 0,48 \\
\hline WG & $\begin{array}{c}\mathbf{W}^{\star \star \star} \\
\mathrm{T}^{\star \star}\end{array}$ & $\begin{array}{r}-0,79 \\
0,58\end{array}$ & 0,62 & $\begin{array}{c}W^{\star \star \star} \\
\log T^{\star \star \star} \\
\log N^{\star \star \star}\end{array}$ & $\begin{array}{r}-0,73 \\
0,61 \\
-0,69\end{array}$ & 0,56 & $\log W^{*}$ & $-0,35$ & 0,12 & $\begin{array}{l}\mathbf{W}^{\star \star \star} \\
T^{\star \star \star}\end{array}$ & $\begin{array}{r}-0,64 \\
0,51\end{array}$ & 0,40 \\
\hline
\end{tabular}

Die Messungen des Winterhalbjahres wurden jeweils der vorangestellten Hauptfrucht zugeordnet. $\mathrm{r}=$ Korrelationskoeffizient der einfachen linearen Korrelation;

$r^{2}=$ Bestimmtheitsmaß der in die multiple Regression mit eingeflossenen Einflußfaktoren;

Einflußfaktor (fett) $\Rightarrow$ in multiple Regression mit eingeflossen;

$\mathrm{W}=$ WFPS; $\mathrm{T}=$ Bodentemperatur; $\mathrm{N}=$ Bodennitrat;

$* \mathrm{p}<0,05 ; * * \mathrm{p}<0,01 ; * * * \mathrm{p}<0,001$ 


\subsubsection{Kumulierte Methanflüsse}

In Tabelle 3-9 sind die kumulierten $\mathrm{CH}_{4}$-Aufnahmeraten bezogen auf das Sommerund Winterhalbjahr sowie auf das Gesamtjahr (März - Februar) der Jahre 1995/96 und 1996/97 dargestellt. Im Versuchsmittel wurde in der Rotation zwischen 450 und $570 \mathrm{~g} \mathrm{CH}_{4}-\mathrm{C} \mathrm{ha}^{-1} \mathrm{a}^{-1}$, vom ungedüngten Grasland lediglich $360 \mathrm{~g} \mathrm{CH}_{4}-\mathrm{C}$ ha $^{-1} \mathrm{a}^{-1}$ aufgenommen.

Tab. 3-9: Kumulierte $\mathrm{CH}_{4}$-C-Aufnahme in den Sommer- und Winterhalbjahren sowie für das Gesamtjahr 1995/96 und 1996/97 (März - Februar).

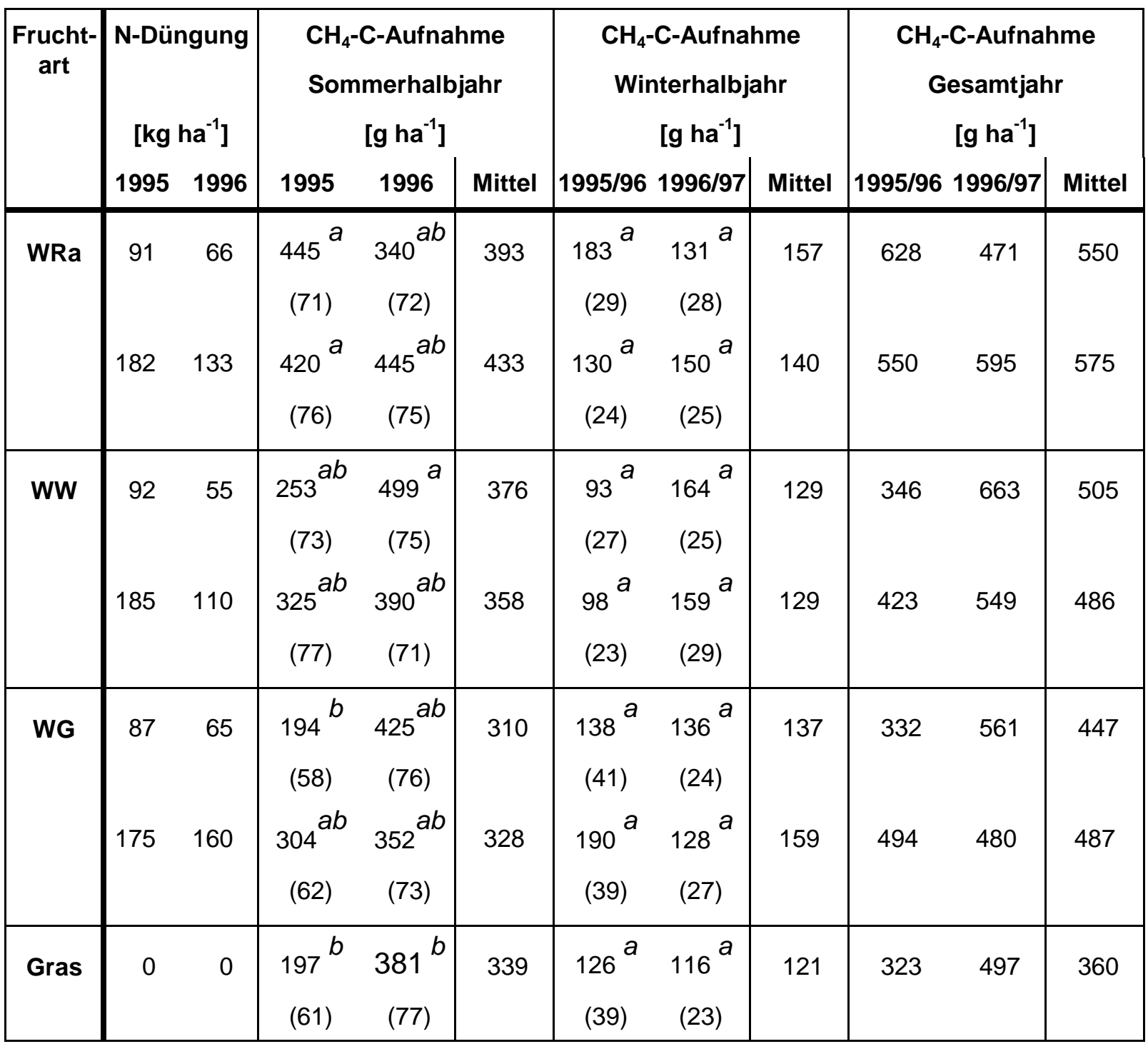

Die Messungen des Winterhalbjahres wurden jeweils der vorangestellten Hauptfrucht zugeordnet.

( ):

Indexbuchstaben: prozentuale Anteile der Sommer- bzw. Winteremissionen am Gesamtjahr. kennzeichnen signifikante Unterschiede $(\mathrm{p}<0,05)$ zwischen den Fruchtarten innerhalb des jeweiligen Halbjahres. 
Über $70 \%$ der jährlichen Methanaufnahme fand in den Sommerhalbjahren statt. Deutliche fruchtartspezifische Effekte, wie sie bei den Lachgasemissionen zu beobachten waren, ließen sich bei der Methanoxidation nicht nachweisen. Im Sommerhalbjahr 1995 zeigten sich zwar signifikante Unterschiede zwischen den Kulturen, diese bestätigten sich jedoch im darauffolgenden Sommerhalbjahr nicht. Die tendenziell niedrigsten Aufnahmeraten des ungedüngten Graslandes dürften auf die höhere Lagerungsdichte dieser Fläche zurückzuführen sein (keine lockernde Bodenbearbeitungsmaßnahmen) (vgl. Tab. 3-1). Eine Verminderung der Ammoniumnitratdüngung um $50 \%$ führte zu keinem signifikanten Anstieg der kumulierten halbjährlichen Methanaufnahme.

\subsection{Diskussion}

\subsection{1 $\quad \mathrm{N}_{2} \mathrm{O}$-Emissionen des Bodens}

DAVIDSON (1991) macht deutlich, daß die Höhe der $\mathrm{N}_{2} \mathrm{O}$-Emissionen maßgeblich vom Vorhandensein von Ammonium und Nitrat im Boden bestimmt wird. Da sich unterschiedliche Stickstoffaneignungsvermögen verschiedener Fruchtarten direkt auf den $\mathrm{N}_{\text {min }}$-Gehalt des Bodens auswirken (LICKFETT, 1993), ist zu vermuten, daß es zu fruchtartspezifischen $\mathrm{N}_{2} \mathrm{O}$-Emissionen kommen kann. Auch eine unterschiedliche Mineralisierbarkeit der Erntereste verschiedener Fruchtarten kann zu unterschiedlichen $\mathrm{N}_{\text {min }}$-Gehalten im Boden führen. LICKFETT (1993) berichtete, daß es unabhängig von der Produktionsintensität nach dem Anbau von WRaps im Vergleich zum Getreide im Herbst und Winter zu erhöhten $\mathrm{N}_{\min }$-Gehalten im Boden $\left(>100 \mathrm{~kg} \mathrm{ha}^{-1} 90 \mathrm{~cm}^{-1}\right.$ ) kam. Es ist deshalb zu vermuten, daß es gerade nach dem Anbau von WRaps unabhängig von der $\mathrm{N}$-Düngeintensität zu erhöhten $\mathrm{N}_{2} \mathrm{O}$ Emissionen kommt. Sowohl FINDLAY und MCKENNEY (1979) als auch VAN CLEEMPUT et al. (1992) wiesen zwar auf fruchtartspezifische $\mathrm{N}_{2} \mathrm{O}$-Emissionen beim Anbau von Klee, Luzerne und Mais hin, jedoch leiteten sie ihre Erkenntnisse nur aus einer kleinen Anzahl von Messungen ab. Werden kurzfristig durchgeführte $\mathrm{N}_{2} \mathrm{O}$ Messungen auf das gesamte Jahr interpoliert, kann dieses zu einer starken Überbzw. Unterschätzung der kalkulierten $\mathrm{N}_{2} \mathrm{O}$-Jahresemissionen führen. Es gibt bis heute keine Langzeituntersuchungen über $\mathrm{N}_{2} \mathrm{O}$-Flüsse in einer Rapsfruchtfolge unter Berücksichtigung einer differenzierten N-Düngung. 


\section{Fruchtartspezifische $\mathrm{N}_{2} \mathrm{O}$-Emissionen}

Die in dieser Arbeit durchgeführten Untersuchungen zeigen, daß der Einfluß einer Fruchtart auf die $\mathrm{N}_{2} \mathrm{O}$-Emissionen nicht auf ihre Vegetationszeit beschränkt ist, sondern im besonderen Maße auch das Winterhalbjahr der Folgefrucht umfaßt. Die von FLESSA et al. (1998) auf dem Versuchsstandort "Scheyern“ durchgeführten Versuche bestätigen dies. Es ist aber zu berücksichtigen, daß die Dauer und Intensität der Vorfruchtwirkung nicht nur abhängig von der vorangestellten Fruchtart, sondern auch durch Bodenbearbeitungsmaßnahmen und die Folgefrucht beeinflußt sein dürfte. Dieses zeigt sich besonders nach WRaps, dessen Erntereste ein engeres $\mathrm{C} / \mathrm{N}-$ Verhältnis $(<50)$ als Getreidestroh aufwiesen.

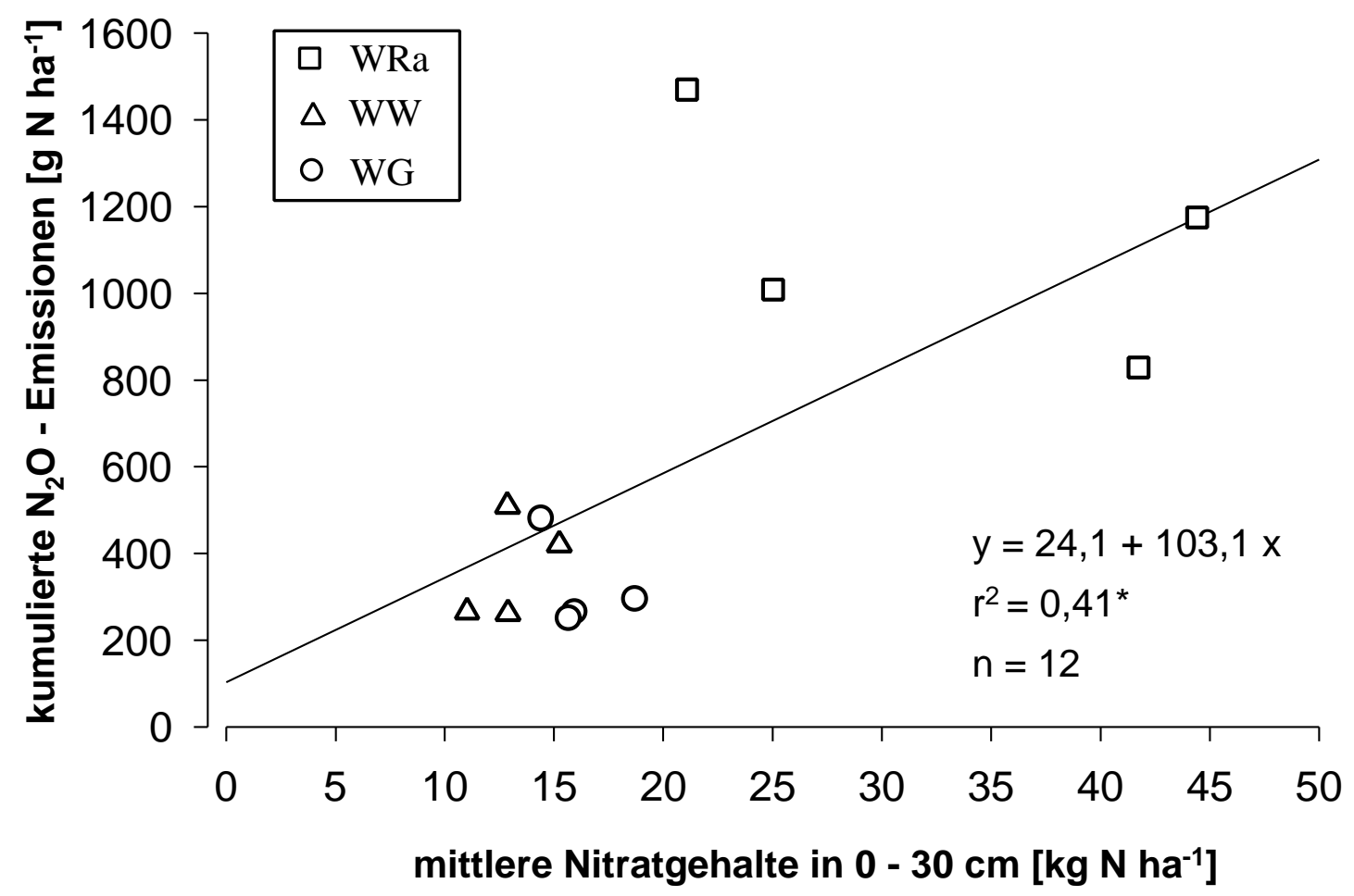

Abb. 3-16: Einfluß der mittleren Nitratgehalte auf die kumulierten $\mathrm{N}_{2} \mathrm{O}-\mathrm{N}-$ Emissionen in den Winterhalbjahren des Versuchszeitraumes unter Berücksichtigung aller Fruchtarten. Signifikanzniveau $\mathrm{p}<0,05$.

Für alle Fruchtarten der Fruchtfolge konnte für die Winterhalbjahre zwar ein signifikant positiver linearer Zusammenhang zwischen den mittleren Nitratgehalten des Bodens und den halbjährlich kumulierten $\mathrm{N}_{2} \mathrm{O}$-Emissionen nachgewiesen werden (Abb. 3-16), dem jedoch nur ein unbefriedigendes Bestimmtheitsmaß $\left(r^{2}=\right.$ 0,41) zukam. Auch Mosier et al. (1993), Lind (1995), Murakami (1987), EATON und 
PATRIQUIN (1993) und MATSON et al. (1990) beschrieben diesen Effekt und führten inn darauf zurück, daß steigende Nitratgehalte im Boden das $\mathrm{N}_{2} \mathrm{O} / \mathrm{N}_{2}$-Verhältnis vergrößern und gleichzeitig die Denitrifikation fördern. Eine Grundvoraussetzung hierfür sei jedoch ein hoher Bodenwassergehalt (RYDEN, 1993, und SKIBA et al., 1992). Die im Vergleich zu den Sommerhalbjahren hohen Bodenwassergehalte (vgl. Tab. 3-5 und 3-6) der Winterhalbjahre dürften neben gleichzeitig erhöhten Nitratgehalten der Grund dafür sein, daß sich auf dem Reinshof nur für die Winterhalbjahre ein Zusammenhang zwischen den Nitratgehalten und den $\mathrm{N}_{2} \mathrm{O}$ Emissionen nachweisen ließ.

Staley et al. (1990), Hansen et al. (1993), Ruser et al. (1998) sowie Flessa und BEESE (1995) führen die Bodenbearbeitung und die Einarbeitung der Ernterückstände als Gründe für gesteigerte $\mathrm{N}_{2} \mathrm{O}$-Emissionen an, da diese Maßnahmen zu einer Erhöhung des N-Umsatzes im Boden führten. Auf dem Reinshof zeigten sich allein beim Anbau von Winterraps in den Winterhalbjahren 1995/96 und 1996/97 im Vergleich zu den anderen Fruchtarten signifikant höhere $\mathrm{N}_{2} \mathrm{O}$-Emissionen (Tab. 3-7) bei vergleichsweise hohen Nitratgehalten in $0-30 \mathrm{~cm}$ Bodentiefe (Abb. 3-8 und 3-9). Nach LICKFETT (1993) sind die erhöhten Nitratgehalte auf die Vorfruchtwirkung des WRapses zurückzuführen. Untersuchungen von LICKFETT (1993) und SCHEFFER (1993) haben gezeigt, daß bei ordnungsgemäßer und extensiver N-Düngung nach der Ernte von WRaps im Vergleich zu Getreide z.T. deutlich erhöhte Nitratgehalte im Boden auftraten $\left(100 \mathrm{~kg} \mathrm{ha}^{-1} 90 \mathrm{~cm}^{-1}\right.$ und mehr). LICKFETT und PRZEMECK (1997) führen dies auf die Mineralisation der Ernte- und Wurzelrückstände des Raps zurück. KAISER et al. (1997) begründen hohe $\mathrm{N}_{2} \mathrm{O}$ Winteremissionen nach Zuckerrüben mit einem engen $\mathrm{C} / \mathrm{N}$ - Verhältnis der Erntereste.

Um hohe $\mathrm{N}_{2} \mathrm{O}$-Emissionen nach WRaps zu vermeiden, müssen daher Nitratgehalte im Winter nach Raps reduziert werden. LICKFETT (1993) schlägt hierzu Änderungen im Bodenbearbeitungs- und Fruchtfolgekonzept vor.

\section{Wichtige Einflußgrößen auf die $\mathrm{N}_{2} \mathrm{O}$-Abgaberaten}

Nach Robertson (1989) und Granl und BockMAN (1994) sind die Bodentemperatur, der Wassergehalt des Bodens sowie der $\mathrm{NO}_{3}$-Gehalt des Bodens maßgebliche Steuergrößen für die $\mathrm{N}_{2} \mathrm{O}$-Abgaberaten eines Standortes. In den vorliegenden Untersuchungen trugen diese Parameter unter den Fruchtarten WRaps, WWeizen und WGerste nur gering zur Klärung der zeitlichen Variabilität der $\mathrm{N}_{2} \mathrm{O}$-Flüsse (Tab. 3-6) bei. Dieses läßt sich mit dem im Vergleich zu anderen 
Standorten (FLESSA et al., 1998) niedrigen Emissionsniveau des Standortes Reinshof (vgl. Abb. 3-10, 3-11 und 3-12) während der beiden Versuchsjahre erklären.

Das DBU Verbundprojekt „Freisetzung und Verbrauch der klimarelevanten Spurengase $\mathrm{N}_{2} \mathrm{O}$ und $\mathrm{CH}_{4}$ beim Anbau nachwachsender Rohstoffe" (FLESSA et al., 1998), in dessen Rahmen auch die vorliegenden Untersuchungen durchgeführt wurden, läßt einen Vergleich des Emissionsniveaus verschiedener Standorte aufgrund des parallelen Anbaus gleicher Fruchtarten bei differenzierter N-Düngung zu (Abb. 3-15). Die mittleren annuellen $\mathrm{N}_{2} \mathrm{O}$-Emissionen der Jahre 1995 bis 1997 unter und nach Winterweizen auf dem Versuchsstandort „Scheyern“ (lößüberlagerte Braunerde, $40 \mathrm{~km}$ nördlich von München) (vgl. FLESSA et al., 1998) waren im Mittel ca. 3,5 mal höher als auf dem Reinshof (Abb. 3-15). Die zeitliche Variation der $\mathrm{N}_{2} \mathrm{O}$ Emissionen wies dort eine bessere Korrelation mit der Bodentemperatur, den Bodenwassergehalten und den Nitratgehalten des Bodens auf (vgl. FLESSA et al., 1998). Auch der Boden des Versuchsstandortes „Canstein“, ein weiterer Standort dieses Projektes (Pseudogley-Braunerde, $30 \mathrm{~km}$ westlich von Kassel), wies unter WRaps bei gleicher N-Düngung im Versuchsjahr 1996/97 ebenfalls deutlich höhere jährliche $\mathrm{N}_{2} \mathrm{O}$-Emissionen als Reinshof auf (vgl. Abb. 3-15).

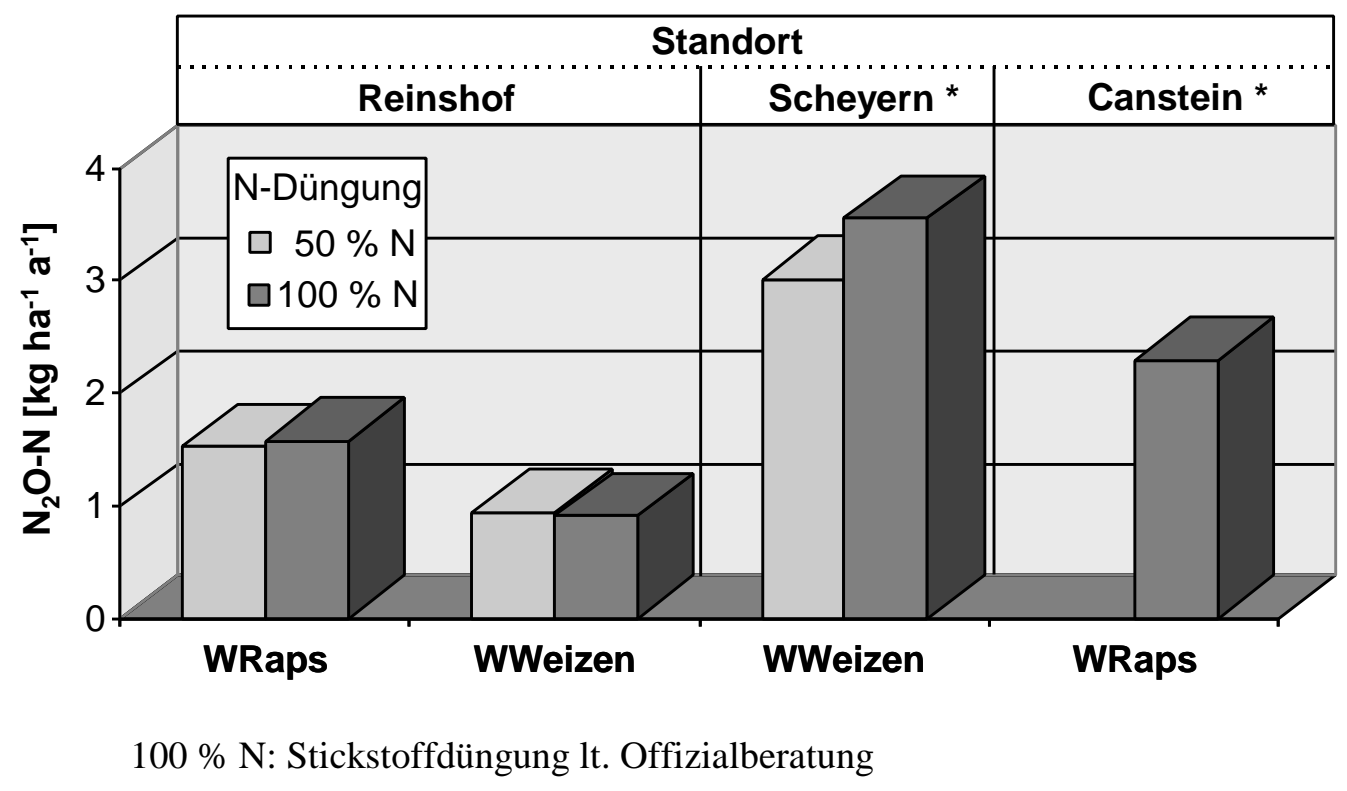

Abb. 3-15: $\quad \mathrm{N}_{2} \mathrm{O}$ - Jahresemissionen der Versuchsstandorte Reinshof und Scheyern (1995 bis 1997) sowie Canstein (1996/97). （* verändert nach: FLESSA et al., 1998)

Das wassergefülte Porenvolumen des Bodens lieferte in multiplen Regressionsrechnungen für alle Fruchtarten auf dem Reinshof keine signifikanten bzw. nur unbefriedigende Korrelationen $\left(r^{2}=<0,31\right)$ zur Beschreibung der zeitlichen 
Variabilität von $\mathrm{N}_{2} \mathrm{O}-$ Emissionen (Tab. 3-6). In Untersuchungen von ROLSTON et al. (1978), Denmead et al. (1979), RYDEN und Lund (1980), ConRAD et al. (1983) und MOSIER et al. (1986) wurden hingegen positive Korrelationen zwischen $\mathrm{N}_{2} \mathrm{O}$ Emissionen und Bodenwassergehalten ermittelt.

Auch stärkere Niederschlagsereignisse ( $>15 \mathrm{~mm}$ tägl.) führten auf dem Reinshof (vgl. Abb. 3-2) kurzfristig nur zu unwesentlich erhöhten $\mathrm{N}_{2} \mathrm{O}$-Emissionen. In Untersuchungen von MOSIER und HUTCHINSON (1981), CATES und KEENEY (1987), HANSEN et al. (1993) und FLESSA et al. (1995) wurden hingegen erhöhte $\mathrm{N}_{2} \mathrm{O}$ Emissionen nach starken Niederschlägen oder nach Beregnungsmaßnahmen gefunden. Als Grund für diese Emissionsereignisse wird von MOSIER und HUTCHINSON (1981) eine reduzierte $\mathrm{O}_{2}$-Diffusion in den Boden angenommen, wodurch die Denitrifikation begünstigt sei. LINN und DORAN (1984) wiesen nach, daß es im Boden bei einem wassergefüllten Porenvolumen $>60 \%$ zu einer Abnahme der Respiration sowie zu einer Zunahme der $\mathrm{N}_{2} \mathrm{O}$-Produktion als Folge einer diffusionsbedingten Abnahme der $\mathrm{O}_{2}$-Verfügbarkeit kam.

Ende Mai 1995 nahm auf den Versuchsflächen des Reinshof nach einer längeren Austrocknungsphase (WFPS $<60 \%$ ) das wassergefüllte Porenvolumen infolge von Niederschlägen auf $>70 \%$ zu (Abb. 3-6). Trotzdem stiegen die $\mathrm{N}_{2} \mathrm{O}$-Emissionsraten unter den 3 Fruchtarten nach dieser Wiederbefeuchtung nur unwesentlich auf ca. $30 \mu \mathrm{g} \mathrm{N}_{2} \mathrm{O}-\mathrm{N} \mathrm{m}^{-2} \mathrm{~h}^{-1}$ an (Abb. 3-10). MOSIER et al. (1986) sowie RUdAZ et al. (1991) beschreiben hingegen deutlich ausgeprägte $\mathrm{N}_{2} \mathrm{O}$-Emissionspeaks nach der Wiederbefeuchtung ausgetrockneter Böden; dies wird von FIRESTONE und DAVIDSON (1989) sowie von RUDAZ et al. (1991) vor allem auf Denitrifikation zurückgeführt.

Die Denitrifikation ist ein anaerober Prozeß, der maßgeblich durch den respiratorischen $\mathrm{O}_{2}$-Verbrauch im Zuge der Zersetzung leicht verfügbarer organischer Substanz sowie durch die Sauerstoffnachlieferung aus der Atmosphäre beeinflußt wird. Nach FLESSA und BEESE (1995) können erhöhte Bodenwassergehalte aufgrund einer reduzierten $\mathrm{O}_{2}$-Diffusion in den Boden hinein Auslöser für eine gesteigerte Denitrifikation sein. Da nicht alle Denitrifizierer Nitrat vollständig bis zum $\mathrm{N}_{2}$ reduzieren, kann es nach GRANLI und BOCKMANN (1994) in Bereichen partiell zunehmender Sauerstoffverfügbarkeit zu einer unvollständigen Nitratreduktion und somit zu verstärkten $\mathrm{N}_{2} \mathrm{O}$-Emissionen kommen.

Auch für den Reinshof waren bei hohem WFPS (> $60 \%$ ) sowie nach Wiederbefeuchtungsereignissen höhere denitrifikationsbürtige $\mathrm{N}_{2} \mathrm{O}$-Emissionen zu erwarten, was sich jedoch nicht bestätigte. Ein Grund hierfür könnte sein, daß der Gley-Auenboden zwar eine sehr feinkörnige Textur aufweist (Tab. 3-1), sich gleichzeitig jedoch durch eine auffallend starke Aggregierung auszeichnet. 
Besonders in den Sommermonaten begünstigt die Ausbildung zahlreicher tiefer Schrumpfrisse bis in eine Tiefe von zum Teil $>6 \mathrm{dm}$ die Infiltration von Niederschlägen und den Gasaustausch, so daß es nur bedingt zu denitrifikativen $\mathrm{N}_{2} \mathrm{O}$-Verlusten des Oberbodens kommen kann. In einem Laborversuch wiesen TORBERT und WOOD (1992) nach, daß bei gleichbleibendem wassergefülltem Porenvolumen, aber gleichzeitiger Erhöhung des Grobporenanteils die gasförmigen $\mathrm{N}$-Verluste reduziert wurden. Dies zeigt, daß die gasförmigen $\mathrm{N}$-Verluste nicht nur vom Anteil des wassergefüllten Porenvolumens, sondern ebenso von der Porengrößenverteilung und -struktur abhängen. Zudem wiesen auf dem Reinshof während des gesamten Versuchszeitraumes lediglich 6 Tage Niederschlagsmengen $>20 \mathrm{~mm} \mathrm{~d}^{-1}$ auf. Starkregenereignisse, die in kurzer Zeit zu einer starken Wiedervernässung mit den oben genannten Folgen für die $\mathrm{N}_{2} \mathrm{O}$-Bildung führen könnten, waren also selten. FLESSA et al. (1998) berichten, daß auf dem Versuchsstandort Scheyern nahe München während des gleichen Versuchszeitraumes doppelt so viele Niederschlagsereignisse mit einer Intensität $>20 \mathrm{~mm} \mathrm{~d}^{-1}$ auftraten. Bei $20 \%$ höheren Gesamtniederschlägen als auf dem Standort Reinshof zeigten sich deutliche Zusammenhänge zwischen der Niederschlagsmenge und dem $\mathrm{N}_{2} \mathrm{O}$-Emissionsniveau.

CONRAD et al. (1983), BENKISER et al. (1987) und SCHNEIDER (1991) beschreiben deutlich positive Korrelationen zwischen der Bodentemperatur und der $\mathrm{N}_{2} \mathrm{O}$-Bildung aus Nitrifikation oder Denitrifikation. In den eigenen Untersuchungen wurden keine bzw. nur sehr schlechte Korrelationen zwischen den Bodentemperaturen in 2,5 cm Bodentiefe und den $\mathrm{N}_{2} \mathrm{O}$-Flüssen unter den 3 Fruchtarten nachgewiesen (Tab. 3-6). Die höchsten Winteremissionen traten aber in den Frost-Tau-Zyklen des Bodens auf (Abb. 3-4 und 3-5 sowie 3-10 und 3-11). Dieses Phänomen wurde zwar von Christensen und Tiedje (1990), Flessa et al. (1995), KAmp und SteindL (1997), RUSER et al. (1996) und SOMMERFELD et al. (1993) mehrfach beschrieben, jedoch sind die Ursachen nicht vollständig geklärt. Biologische und physikalische Prozesse werden als Ursache für die $\mathrm{N}_{2} \mathrm{O}$-Freisetzung in Tauperioden diskutiert.

Im Winterhalbjahr 1995/96 waren die Frost-Tau-Zyklen auffallend stark ausgeprägt (Abb. 3-4). In den Auftauphasen, in denen sich Wasser aus der geschmolzenen Schneedecke bis zu einem Zentimeter hoch auf dem noch gefrorenen Boden staute (Ende Februar 1996), kam es zu besonders hohen Emissionsereignissen sowohl nach WRaps als auch nach WWeizen. Höchste $\mathrm{N}_{2} \mathrm{O}$-Emissionen wurden auch in der Auftauphase des Bodens Ende Januar 1997 wiederholt nach WRaps unter WWeizen gemessen (Abb. 3-5 und 3-11 (c)). Der Boden taute nicht nur von oben nach unten, sondern auch von der unteren Frostgrenze in Richtung Bodenoberfläche. Es wird vermutet, daß es infolge des Wiederauftauens zu einer 
erhöhten Verfügbarkeit an leicht abbaubaren Kohlenstoffverbindungen kam, wodurch die Aktivität heterotropher Mikroorganismen und somit die $\mathrm{O}_{2}$-Zehrung im Boden anstieg. Da das an der Oberfläche gestaute Wasser zusätzlich eine Diffusionsbarriere für atmosphärischen Sauerstoff darstellt, dürften die hohen $\mathrm{N}_{2} \mathrm{O}$ Emissionsereignisse zu diesem Zeitpunkt aufgrund eines stark abgesunkenen Sauerstoffpartialdruckes denitrifikativer Herkunft sein. Spezielle Untersuchungen wurden zur Klärung dieser Frage nicht durchgeführt.

Weiter wird vermutet, daß leicht wasserlösliches $\mathrm{N}_{2} \mathrm{O}$ wahrscheinlich an der wassergesättigten oder wasserüberstauten Bodenoberfläche in Lösung geht, bevor es anschließend aus der Lösungsphase in die atmosphärische Luft freigesetzt wird. Die Nitratnachlieferung dürfte während der Auftauphase sowohl auf eine Mobilisierung aus der aufgetauten mortalen Biomasse als auch aus Ernterückständen zurückzuführen sein.

Hohe, auf Frost-Tau-Effekte zurückzuführende $\mathrm{N}_{2} \mathrm{O}$-Emissionen zeigten sich unter WWeizen (Abb. 3-11(a)) auch noch im März 1996 (= Sommerhalbjahr 1996). Gefördert wurden diese hohen Emissionen jedoch nicht nur durch erhöhte Nitratgehalte, z.B. infolge des WRaps-Anbaus (Abb. 3-9(a)), sondern zusätzlich durch die erste Frühjahrs-N-Düngung zu WWeizen, die vor Ende der Frostperiode ausgebracht worden war. Ein starkes, aber kurzes Emissionsereignis (Abb. 3-11 (b)) zeigte sich ebenfalls infolge der 1. Frühjahrs-N-Gabe zur WGerste Mitte März 1996. Diese hohen Emissionen dürften neben den Frost-Tau-Effekten ausschließlich mit den erhöhten Nitratgehalten infolge der Düngung zu erklären sein. DuXBURY et al. (1982) und DUXBURY (1994) berichten von steigenden $\mathrm{N}_{2} \mathrm{O}$-Emissionen nach hoher Stickstoffdüngung.

\section{Einfluß der N-Düngung auf die $\mathrm{N}_{2} \mathrm{O}$-Emissionen}

Eine Reduzierung der N-Düngung um $50 \%$ in der WRaps-WWeizen-WGersteFruchtfolge führte weder in den Sommer- noch in den Winterhalbjahren zu einer signifikanten Reduktion der $\mathrm{N}_{2} \mathrm{O}$-Emissionen (Tab. 3-7). Parallel dazu kam es zu keiner Verringerung der Nitratgehalte des Bodens (vgl. Kap. 3.3.1.2). LICKFETT (1993) sowie LICKFETT und PRZEMECK (1997) berichten, daß eine Verminderung der Stickstoffdüngung bei WRaps zu keiner entsprechenden Reduzierung der Nitratgehalte des Bodens im Herbst und Winter nach der Ernte führen, sofern die Grundsätze einer „ordnungsgemäßen Bewirtschaftung“ als obere Düngungsgrenze eingehalten werden. Auch KAISER et al. (1997) fanden beim Anbau verschiedener Fruchtarten für die Winterhalbjahre keine lineare Beziehung zwischen den $\mathrm{N}_{2} \mathrm{O}$ Verlusten und einer Reduktion der N-Düngung (N $100 \%$ (=ordnungsgemäß), N 50 \% 
und $\mathrm{N} 0$ ). Lediglich für die Vegetationszeit deutete sich ein schwacher Zusammenhang an. Diese Ergebnisse stehen im Widerspruch zu Untersuchungen von EICHNER (1990) UND BOUWMAN (1994), die eine generelle Abhängigkeit der $\mathrm{N}_{2} \mathrm{O}$ Emission von der applizierten Düngermenge fanden. Auch auf den Versuchsflächen in Scheyern wurde ein deutlicher N-Düngereffekt festgestellt (FLESSA et al., 1998).

Auf dem seit ca. 10 Jahren ungedüngten Grasland des Reinshof (Tab. 3-7) traten mit $0,34 \mathrm{~kg} \mathrm{~N}_{2} \mathrm{O}-\mathrm{N} \mathrm{ha}^{-1} \mathrm{a}^{-1}$ die geringsten $\mathrm{N}_{2} \mathrm{O}$-Emissionen auf; sie können als Basisemissionen dieses Standortes angenommen werden.

BouWmaN (1994) schätzt die düngungsinduzierte Emission von $\mathrm{N}_{2} \mathrm{O}$ anhand einer Literaturauswertung auf 1,25\% der applizierten N-Menge unter der Annahme, daß ungedüngte Flächen im Mittel eine Basisemission von $1 \mathrm{~kg} \mathrm{~N}_{2} \mathrm{O}-\mathrm{N} \mathrm{ha}^{-1} \mathrm{a}^{-1}$ zeigen. Diese von Bouwman dargestellte Beziehung stellt zur Zeit die wichtigste Grundlage für die Abschätzung der globalen Emission aus landwirtschaftlich genutzten Böden dar (IPCC, 1996). Der Autor weist aber darauf hin, daß die Beziehung sich nicht dazu eignet, die Emissionen unterschiedlicher Fruchtarten zu schätzen.

Eine nach Bouwman (1994) nachvollzogene Berechnung (vgl. Kap. 2.1.3) der düngungsbezogenen $\mathrm{N}_{2} \mathrm{O}$-Emissionen unter Berücksichtigung der Basisemission des Reinshof $\left(0,34 \mathrm{~kg} \mathrm{ha}^{-1} \mathrm{a}^{-1}\right)$ variiert je nach Fruchtart, Versuchsjahr und Düngungsintensität zwischen 0,2 und 2,1\% (Tab. 3-10). In allen Fruchtarten führte die Reduktion der Düngung zu einer relativen Erhöhung der düngungsbezogenen $\mathrm{N}_{2} \mathrm{O}-\mathrm{N}$-Austräge. Entgegen dem mittleren Emissionsfaktor von $1,25 \%$ der

Tab. 3-10: Düngungs- und ertragsbezogene annuelle $\mathrm{N}_{2} \mathrm{O}-\mathrm{N}-$ Emissionen (März bis Februar) (abzüglich der Basisemissionen aus dem ungedüngten Grasland).

\begin{tabular}{|c|c|c|c|c|c|c|c|c|}
\hline \multirow[t]{2}{*}{$\begin{array}{c}\text { Frucht- } \\
\text { art }\end{array}$} & \multicolumn{2}{|c|}{$\begin{array}{l}\text { N-Düngung } \\
{\left[\mathrm{kg} \mathrm{ha}^{-1}\right]}\end{array}$} & \multicolumn{2}{|c|}{$\begin{array}{c}\mathrm{N}_{2} \mathrm{O}-\mathrm{N} \text { in \% } \\
\text { des Dünger-N }\end{array}$} & \multicolumn{2}{|c|}{$\begin{array}{l}\text { Kornertrag } \\
{\left[\mathrm{dt} \mathrm{TM} \mathrm{ha}{ }^{-1}\right]}\end{array}$} & \multicolumn{2}{|c|}{$\begin{array}{c}\mathrm{N}_{2} \mathrm{O}-\mathrm{N} / \text { Ertragsrelation } \\
{\left[\mathrm{g} \mathrm{dt}^{-1}\right]}\end{array}$} \\
\hline & 1995 & 1996 & $1995 / 96$ & $1996 / 97$ & 1995/96 & $1996 / 97$ & $1995 / 96$ & 1996/97 \\
\hline \multirow[t]{2}{*}{ WRa } & 91 & 66 & 1,1 & 2,1 & 35,7 & 25,2 & 35 & 72 \\
\hline & 182 & 133 & 0,8 & 0,8 & 33,8 & 33,6 & 51 & 44 \\
\hline \multirow[t]{2}{*}{ WW } & 92 & 55 & 0,4 & 1,6 & 59,5 & 76,9 & 10 & 17 \\
\hline & 185 & 110 & 0,2 & 0,7 & 69,2 & 91,1 & 9 & 13 \\
\hline \multirow[t]{2}{*}{ WG } & 87 & 65 & 0,3 & 0,8 & 61,1 & 41,3 & 9 & 22 \\
\hline & 175 & 160 & 0,2 & 0,6 & 69,7 & 55,95 & 8 & 21 \\
\hline
\end{tabular}


applizierten N-Menge nach BouwmaN (1994) ergaben sich im Mittel beider NDüngungsstufen in den untersuchten Fruchtarten Faktoren von 1,2\% für WRaps, $0,73 \%$ für WWeizen und $0,48 \%$ für die WGerste. Der Einfluß der Fruchtart auf die $\mathrm{N}_{2} \mathrm{O}$-Emissionen läßt sich also nicht vereinfachend auf die Höhe der fruchtspezifischen $\mathrm{N}$-Düngung reduzieren. Die Verwendung eines mittleren Emissionsfaktors von 1,25\% (BouwMAN, 1994) hätte zu einer deutlichen Überschätzung der $\mathrm{N}_{2} \mathrm{O}$-Emissionen aus der Getreidefläche geführt.

Eine Verringerung der N-Gaben hatte andererseits in der Regel eine Erhöhung der $\mathrm{N}_{2} \mathrm{O}-\mathrm{N}-$ Verluste je Einheit Ertrag zur Folge (Tab. 3-10), da eine Reduzierung der NGabe zwar i.d.R. zu einem Rückgang der Erträge führte, aber gleichzeitig keinen Rückgang der $\mathrm{N}_{2} \mathrm{O}$-Emissionen bewirkte. FLESSA et al. (1998) konnten für den Versuchsstandort Scheyern nach reduzierter N-Düngung für alle Fruchtarten Ertragsrückgänge nachweisen. Da gleichzeitig die $\mathrm{N}_{2} \mathrm{O}-$ Emissionen im Vergleich zu den Erträgen überproportional zurückgingen, kam es in Scheyern im Gegensatz zum Reinshof i.d.R. nach einer Reduzierung der N-Düngung zu einem deutlichen Rückgang der emittierten $\mathrm{N}_{2} \mathrm{O}-\mathrm{N}$-Mengen je Ertragseinheit (FLESSA et al., 1998).

Neben den in dieser Untersuchung bestimmten direkten $\mathrm{N}_{2} \mathrm{O}$-Emissionen aus den Versuchsflächen können jedoch auch die indirekten $\mathrm{N}_{2} \mathrm{O}$-Emissionen im Felde berücksichtigt werden. Nach Angaben des IPCC (1996) kann von einer indirekten Emission in Höhe von $0,75 \%$ der applizierten Stickstoffmenge ausgegangen werden. Diese werden damit begründet, daß es durch den Austrag von Dünger- $N$ aus landwirtschaftlich genutzten Flächen in den benachbarten terrestrischen oder aquatischen Systemen zu zusätzlichen $\mathrm{N}_{2} \mathrm{O}$-Emissionen kommen kann. Während es in der WRaps-Fruchtfolge des Reinshof bei einer Reduzierung der N-Düngung um $50 \%$ zu keinem signifikanten Rückgang der direkten $\mathrm{N}_{2} \mathrm{O}$-Feldemissionen kam (Tab. 3-7), ist parallel dazu aber von einem rechnerischen Rückgang der indirekten $\mathrm{N}_{2} \mathrm{O}$ Emissionen auszugehen. Die gesamten $\mathrm{N}_{2} \mathrm{O}$-Emissionen biologischen Ursprungs (=Summe aus direkten Feldemissionen und indirekten Emissionen) würden somit bei einer reduzierten N-Düngung auch auf dem Reinshof sinken.

\subsection{2 $\quad \mathrm{CH}_{4}$-Aufnahmen des Bodens}

Die landwirtschaftliche Umwandlung von naturnahen Ökosystemen führt nach KELLER et al. (1990) und DoBbIE et al. (1996) zu einer Abnahme der Methanoxidation terrestrischer Böden. Ob es speziell durch den Anbau von WRaps zu einer zusätzlichen Verringerung der Methanaufnahmeraten eines Standortes kommt, wurde bisher nicht geklärt. In dieser Arbeit soll analog zur Vorgehensweise beim $\mathrm{N}_{2} \mathrm{O}$ 
der Einfluß von Bodentemperatur, WFPS und $\mathrm{N}_{\min }$-Gehalte des Bodens auf die Methanoxidation herausgearbeitet werden. KING und ADAMSON (1992), CRILL et al. (1994) und BORKEN (1996) können keinen eindeutigen Zusammenhang zwischen der Bodentemperatur und der Methanoxidation des Bodens nachweisen. STRIEGEL et al. (1993) betrachten als Haupteinflußfaktor der Methanoxidation die Höhe der Methandiffusion von der Atmosphäre zu den Methanoxidierern im Boden; die Diffusion werde wiederum maßgeblich vom Wassergehalt des Bodens bestimmt. Der vermehrte Einsatz von Stickstoffdüngern in der Landwirtschaft führt nach STEUDLER et al. (1989) Mosier et al. (1991) und HütscH et al. (1994) zu einer Reduktion der Senkenstärke des Bodens.

\section{Wichtige Einflußgrößen auf die $\mathrm{CH}_{4}$-Flußraten}

Deutliche fruchtartspezifische Effekte, wie sie bei den Lachgasemissionen zu beobachten waren, ließen sich bei der Methanoxidation nicht nachweisen, d.h. durch den Anbau von WRaps kam es auf zuvor landwirtschaftlich genutzten Flächen zu keiner zusätzlichen Reduzierung der Methanaufnahmeraten.

Der Versuchsstandort stellte während des gesamten Versuchszeitraumes in allen Versuchsvarianten eine Senke für atmosphärisches Methan dar. CICERONE und OREMLAND (1988) und WATSON et al. (1992) führen dieses auf die Aktivität von methanoxidierenden Mikroorganismen zurück (vgl. Kap. 2.2.2), die damit eine regulierende Funktion im globalen Methankreislauf einnehmen. Die mittleren $\mathrm{CH}_{4}^{-}$ Aufnahmeraten der WRaps-Fruchtfolge waren mit 0,45 bis max. $0,58 \mathrm{~kg} \mathrm{ha}^{-1} \mathrm{a}^{-1} \mathrm{im}$ Vergleich zu allen methanoxidierenden Standorten jedoch relativ gering (Tab. 3-9). BOWDEN et al. (1993) und DoBBIE et al. (1996) beschreiben Methanaufnahmeraten in Waldökosystemen von $>5 \mathrm{~kg} \mathrm{ha}^{-1} \mathrm{a}^{-1}$. Es wird sogar von Senkenstärken von 13 bis $15 \mathrm{~kg} \mathrm{ha}^{-1} \mathrm{a}^{-1}$ berichtet (STEUDLER et al., 1989), jedoch beruhen die zuletzt genannten Zahlen auf Messungen, die ausschließlich während der Vegetationsperiode (Mai bis Oktober) durchgeführt wurden. Da solche Messungen die jahreszeitliche Dynamik der Methanflüsse, wie sie auch auf dem Reinshof deutlich zu erkennen waren (Abb. 3-10 und 3-11), nicht widerspiegeln, ist bei einer Übertragung dieser Daten auf das gesamte Jahr von einer Überschätzung der Senkenstärke auszugehen. Für landwirtschaftlich genutzte Flächen werden Aufnahmeraten zwischen 0,1 und 2,0 kg ha ${ }^{-1} \mathrm{a}^{-1}$ angegeben (MOSIER et al., 1991; LeSSARD et al., 1994; Ambus und Christensen, 1995; FleSSA et al., 1995). Der direkte Vergleich mit dem Standort Scheyern (40 km nördlich von München) zeigte während des gleichen Versuchszeitraumes unter WWeizen in beiden $\mathrm{N}$-Varianten weniger als $50 \%$ der jährlichen Methanaufnahmeraten des Reinshof, unter 
Winterraps in Canstein (30 km westlich von Kassel) waren es im Versuchsjahr 1996/97 ebenfalls nur ca. 55 \% der mittleren annuellen $\mathrm{CH}_{4}$-Oxidationsleistung (Abb. 3-17).

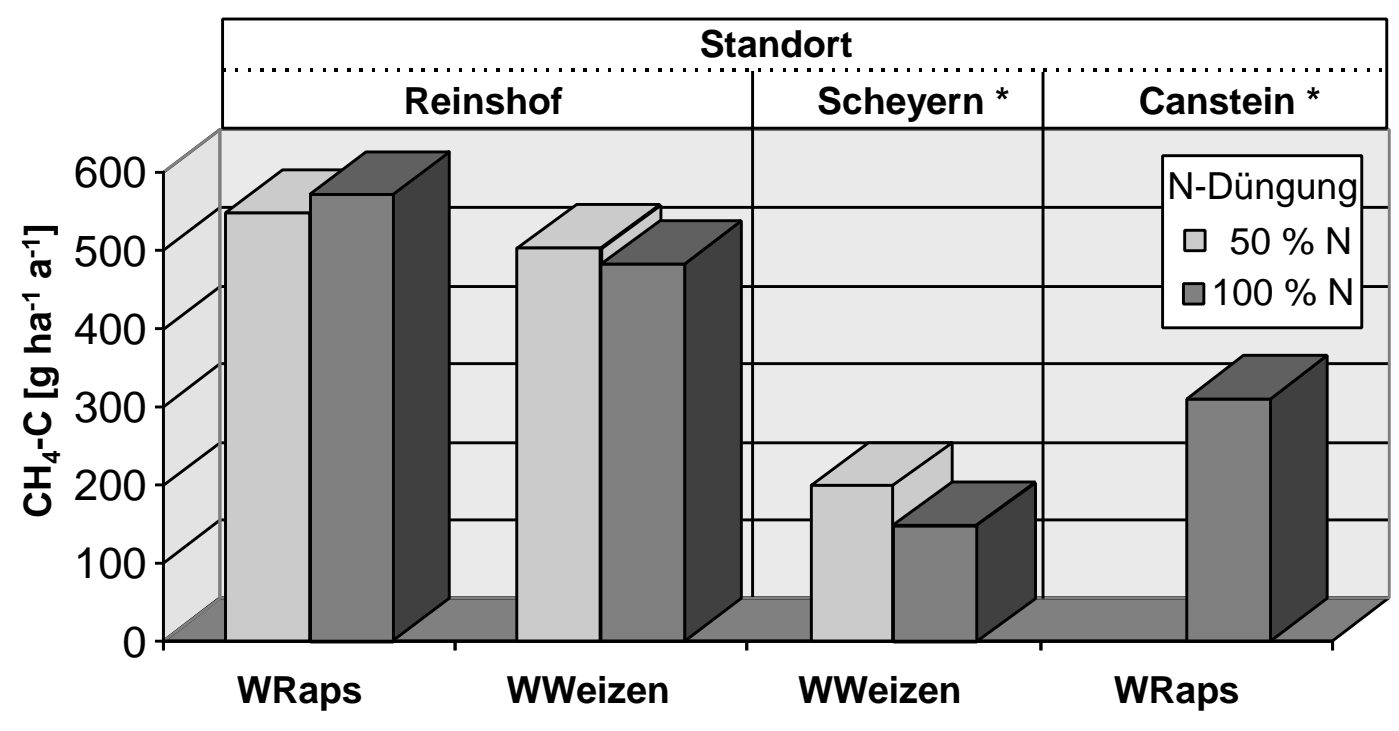

$100 \%$ N: N-Düngung lt. Offizialempfehlung

Abb. 3-17: $\quad \mathrm{CH}_{4^{-}}$Jahresoxidationsleistung der Versuchsstandorte Reinshof und Scheyern (1995 bis 1997) sowie Canstein (1996/97). (*verändert nach: FLESSA et al., 1998)

Die Methanflüsse waren sowohl in den Sommer- als auch in den Winterhalbjahren unter allen Fruchtarten signifikant negativ mit dem WFPS korreliert. Die Bestimmtheitsmaße der multiplen Regression betrugen max. 0,62; im Mittel aller Fruchtarten konnten rund $40 \%$ der Variation der $\mathrm{CH}_{4}$-Aufnahme über die Dynamik des WFPS erklärt werden (vgl. Tab. 3-8). Analog dazu zeigten STEUDLER et al. (1989), WHALEN et al. (1990), DörR et al. (1993), CASTRO et al. (1995) und CZEPIEL et al. (1995), daß die Methanflußrate vom Wassergehalt des Bodens abhängt. Die Ergebnisse dieser Arbeit bestätigen, daß der Gasaustausch im Boden maßgeblich durch das WFPS gesteuert wird. Es wirkt somit als limitierender Faktor für die Substratversorgung $\left(\mathrm{CH}_{4}\right)$ der methanotrophen Mikroorganismen und führt somit zu einer Begrenzung der $\mathrm{CH}_{4}$-Oxidationsleistung des Bodens. Dies ist auf physikalische Vorgänge zurückzuführen, da die Diffusion von Methan in Wasser um den Faktor $10^{4}$ langsamer als in Luft verläuft (MARREDO und MASON, 1972). 
Im Versuchsmittel erfolgten mehr als $70 \%$ der jährlichen Oxidationsleistungen während der Sommerhalbjahre (Tab. 3-9). Während der mittlere WFPS saisonal bedingt in den beiden Sommerhalbjahren des Versuchszeitraumes im Mittel der gesamten Fruchtfolge bei nur ca. $60 \%$ lag, wurden für die Winterhalbjahre im Mittel ca. $71 \%$ berechnet (vgl. Abb. 3-6 und 3-7). In der WRaps-Fruchtfolge konnte für den gesamten Versuchszeitraum ein signifikant negativer Zusammenhang zwischen den mittleren wassergefüllten Porenvolumina und der mittleren Höhe der $\mathrm{CH}_{4}{ }^{-}$ Aufnahmeraten nachgewiesen werden (Abb. 3-18), das Bestimmtheitsmaß lag bei 0,63. Auch BOWDEN et al. (1993), LeSSARD et al. (1994) und AmBus und CHRISTENSEN (1995) berichten von einer Saisonalität der Methanflüsse, die sie vorwiegend auf den Wassergehalt im Boden zurückführen. Auf dem Reinshof kam es in den Winterhalbjahren bei sehr feuchten Bodenverhältnissen (WFPS im Mittel $>76 \%$ ) vereinzelt auch zu geringen Methan-Emissionen. Auch BowDEN et al. (1993) fanden im Winter und Frühjahr geringe Methan-Emissionen, die bei hohem Bodenwassergehalt auf anaerobe Bedingungen hinweisen.

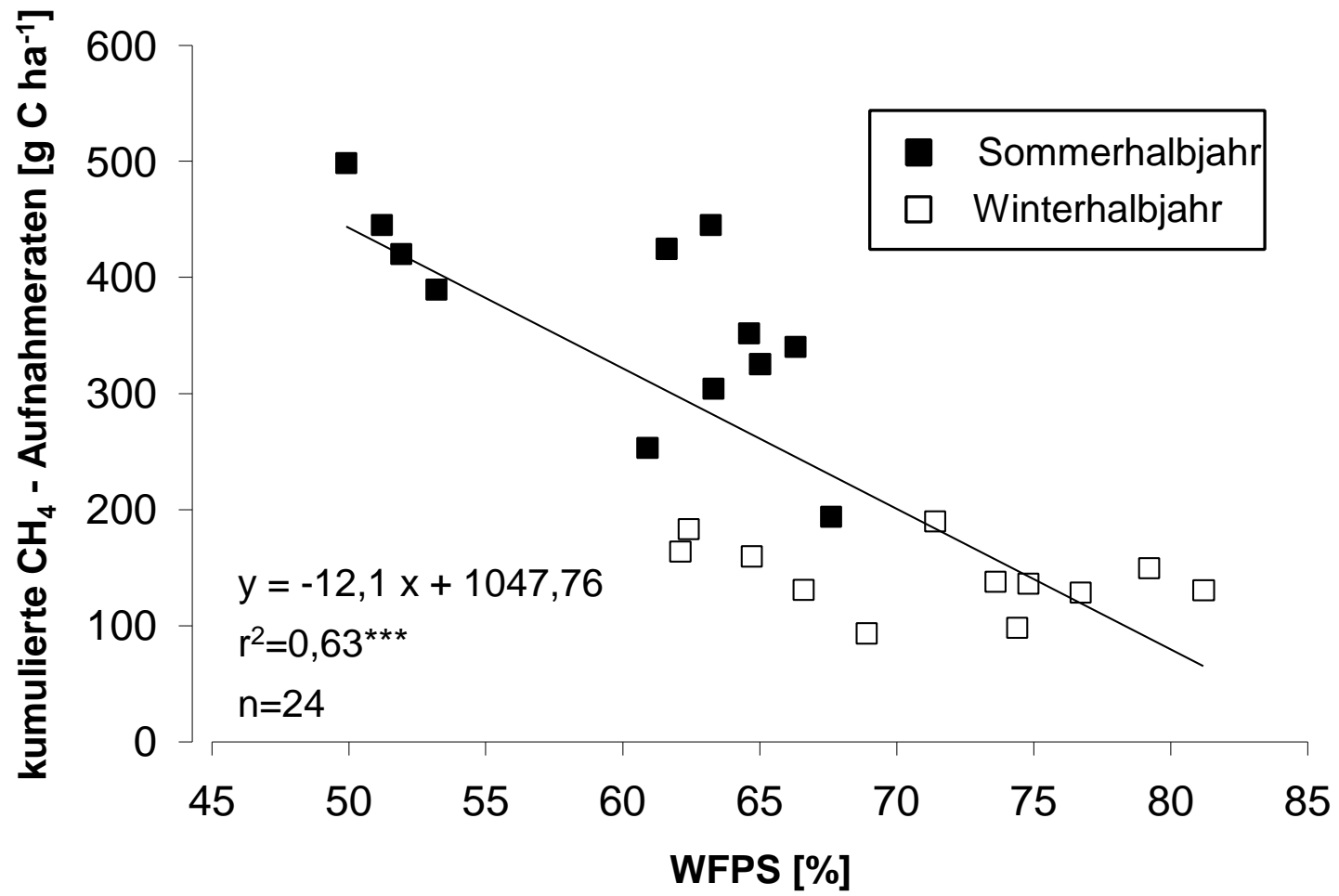

Abb. 3-18: Einfluß des mittleren WFPS auf die kumulierten Methan-Aufnahmeraten in den Sommer- und Winterhalbjahren des Versuchszeitraumes unter Berücksichtigung aller Fruchtarten. Signifikanzniveau $\mathrm{p}<0,05$. 
Die Bodentemperaturen zeigten über den gesamten Versuchszeitraum unter fast allen Fruchtarten signifikant positive Korrelationen zu den Methan-Flußraten (vgl. Tab. 3-8). In Freilanduntersuchungen ist es jedoch nicht möglich, den Einfluß von Bodenfeuchte und Bodentemperatur voneinander zu trennen, da beide Parameter in einer starken Wechselwirkung zueinander stehen. Nach KING und ADAMSON (1992) und ADAMSON und KING (1993) wird die biologische Aktivität der methanotrophen Bakterien jedoch weniger von der Bodentemperatur als von der Methan- und Sauerstoffdiffusion in den Boden beeinflußt. Die Diffusionsmechanismen wiederum werden maßgeblich von den Wassergehalten des Bodens gesteuert, so daß die Bodentemperaturen im Hinblick auf die Methanoxidation eine eher untergeordnete Rolle spielen dürften. Trotzdem geben BOWDEN et al. (1993) die durch die Temperatur zu erklärende Variabilität der Methanoxidation mit $60 \%$ an.

Die Nitratgehalte des Bodens in 0-30 cm Tiefe spielten zur Klärung der zeitlichen Variabilität der Methanflüsse ebenfalls eine nur untergeordnete Rolle (vgl. Tab. 3-8). BORKEN (1996) konnte zur Abschätzung der räumlichen Variabilität mit einem Bestimmtheitsmaß von 0,05 nur tendenziell einen schwach negativen Zusammenhang zwischen den Nitratkonzentrationen der Bodenlösung und der Methanaufnahmerate nachweisen.

\section{Einfluß der $\mathrm{N}$-Düngung auf die $\mathrm{CH}_{4}$-Flußraten}

Nach Reduzierung der N-Düngung um $50 \%$ konnte in allen Versuchsvarianten kein signifikanter Anstieg der Methanaufnahmeraten verzeichnet werden (Tab. 3-9). FLESSA et al. (1995) fanden auf Ackerflächen trotz differenzierter N-Düngung keine nennenswerten Abweichungen zwischen den $\mathrm{CH}_{4}$-Oxidationsraten. MOSIER et al. (1991), HÜTSCH et al. (1994) und STEUDLER et al. (1996) berichten, daß verglichen mit einer Landnutzungsänderung der unmittelbare N-Düngeeffekt in der Regel geringer sei. Auch BORKEN (1996) weist auf einer gedüngten Buchenfläche im Solling in einer dreijährigen Meßperiode nach, daß eine Reduzierung der Ammoniumdüngung um $100 \%$ und der Nitratdüngung um $80 \%$ nicht zu einer erwarteten Erhöhung der Methan-Aufnahmerate führt. Dieses zeigt, daß der Effekt mineralischer Stickstoffdüngung über Jahre hinweg erhalten bleibt. DoBBIE et al. (1996) und OJIMA et al. (1993) zeigten, daß selbst Jahrzehnte nach einer Wiederaufforstung die $\mathrm{CH}_{4}$-Oxidationsrate durch die landwirtschaftliche Vornutzung beeinflußt wurde. Es ist zu vermuten, daß die methanotrophen Bakterienpopulationen, die zuvor durch hohe N-Zufuhr stark dezimiert wurden, sich kurzfristig nicht regenerieren können. PRIEME et al. (1997) vermuten, daß die langfristige 
Regeneration der methanotrophen Organismen auf ihre geringe Wachstumsrate zurückzuführen sei.

Eine kurzfristige Verringerung der $\mathrm{CH}_{4}$-Oxidationsraten, wie sie sich im Sommerhalbjahr 1996 nach den jeweiligen Ammoniumnitrat-Gaben andeuteten (Abb. 3-14), wurden auch von DUNFIELD und KNOWLES (1995) und SCHNELL und KING (1994) beobachtet. Eine Reduktion der Methanoxidation nach Ammoniumapplikation ist wahrscheinlich darauf zurückzuführen, daß die aktiven Zentren der Methanmonooxygenase (MMO) aufgrund einer relativ breiten Substratspezifität auch vom Ammonium belegt werden können (MOSIER et al., 1991; HÜTSCH et al., 1994; SCHNELL Und KING, 1994; DUNFIELD und KNOWLES, 1995; BORKEN, 1996).

Die Grasfläche als ungedüngte Referenzfläche unterschied sich im Hinblick auf die Methanoxidationsleistungen kaum von den gedüngten Fruchtfolgeflächen (Tab. 3-9). Hier zeigt sich erneut die Wirkung der landwirtschaftlichen Vornutzung, eine Begrünung dieser Fläche bei gleichzeitigem Verzicht auf N-Düngung brachte selbst nach ca. 10 Jahren noch keine Erhöhung der Methanaufnahmeraten.

\subsubsection{Klimarelevanz der Produktion der unterschiedlichen Fruchtarten}

Wie in Kapitel 3.2.4 beschrieben, wurde für alle Fruchtarten die Treibhauswirksamkeit der Produktion bilanziert. Die kumulierten Methanaufnahmeraten im Felde wurden in die Bilanzierung nicht mit aufgenommen, da eine anthropogen unbeeinflußte Referenzfläche zur Abschätzung der Basisoxidation von Methan nicht zur Verfügung stand. Auch die ungedüngte Grasfläche schied als Referenzfläche aus, denn es war davon auszugehen, daß die $\mathrm{CH}_{4}$-Oxidationsleistung der "GrasReferenzfläche", die erst vor ca. 10 Jahren aus der landwirtschaftlichen Produktion genommen wurde, durch ehemalige Bewirtschaftung und Nährstoffeinträge beeinflußt war. Die kumulierten Methanaufnahmen in der Fruchtfolge lassen jedoch erkennen, daß der Anbau von WRaps in der Fruchtfolge zu keiner fruchtartspezifischen Veränderung der Methanoxidation führt und daß die kumulierten Werte für die Treibhausbilanz der Produktion ohnehin unbedeutend sind.

Als klimawirksame Emissionen biologischen Ursprungs beim Anbau von WRaps, WWeizen und WGerste (Tab. 3-11) wurden die direkten $\mathrm{N}_{2} \mathrm{O}$-Emissionen aus der Produktionsfläche sowie die indirekten $\mathrm{N}_{2} \mathrm{O}$-Emissionen, die z.B. durch umweltbelastende $\mathrm{N}$-Austräge aus den Produktionsflächen verursacht werden, berücksichtigt (vgl. Kap. 3.2.4). Außerdem flossen die klimawirksamen Emissionen 
energetischen Ursprungs in die Berechnungen mit ein. Unter Berücksichtigung der Klimawirksamkeit der bei der Produktion einzelner Fruchtarten zusätzlich emittierten Gase wurde die relative Treibhauswirksamkeit (vgl. Tabelle 3-4) anhand von $\mathrm{CO}_{2}-$ Äquivalenten dargestellt (Tab. 3-11).

Aufgrund der erhöhten $\mathrm{N}_{2} \mathrm{O}$-Feldemission trat die höchste Treibhauswirksamkeit bei der Produktion von WRaps auf. Sowohl im Vergleich der jeweils intensiv als auch der jeweils extensiv gedüngten Fruchtarten zeigte der WRaps jeweils mit 2728 bzw. $1960 \mathrm{~kg} \mathrm{CO}$-Äquivalenten ha ${ }^{-1} \mathrm{a}^{-1}$ die größte Klimawirksamkeit (Tab. 3-11). Die

Tab. 3-11: Klimawirksame Emissionen (in $\mathrm{CO}_{2}$ - ̈̈quivalenten) beim Anbau von Winterraps, Winterweizen und Wintergerste (Mittel der 2 Jahre).

\begin{tabular}{|c|c|c|c|c|c|c|c|c|}
\hline \multirow[t]{3}{*}{ Fruchtart } & \multirow{3}{*}{$\begin{array}{l}\text { Dünge- } \\
\text { variante }\end{array}$} & \multirow{3}{*}{$\begin{array}{l}\text { N-Düng. } \\
{\left[\mathrm{kg} \mathrm{ha}^{-1}\right]}\end{array}$} & \multicolumn{6}{|c|}{$\mathrm{CO}_{2}$-Äquivalente $\left[\mathrm{kg} \mathrm{ha}^{-1} \mathrm{a}^{-1}\right]$} \\
\hline & & & \multicolumn{2}{|c|}{ biologischer Ursprung } & \multicolumn{3}{|c|}{ energetischer Ursprung } & \multirow[t]{2}{*}{ Total $^{*}$} \\
\hline & & & $\mathrm{N}_{2} \mathrm{O}_{\text {Feld }}$ & $\mathrm{N}_{2} \mathrm{O}_{\text {ind. }}$ & $\mathrm{KS}(\mathrm{D})$ & D & PS & \\
\hline \multirow[t]{2}{*}{ WRaps } & N 50 & 79 & 768 & 296 & 352 & 525 & 19 & 1960 \\
\hline & & & $(39,2)$ & $(15,0)$ & $(18,0)$ & $(26,8)$ & $(1,0)$ & \\
\hline \multirow[t]{2}{*}{ WRaps } & N100 & 158 & 798 & 594 & 353 & 964 & 19 & 2728 \\
\hline & & & $(29,3)$ & $(21,7)$ & $(12,9)$ & $(35,4)$ & $(0,7)$ & \\
\hline \multirow[t]{2}{*}{ WWeizen } & N 50 & 74 & 476 & 277 & 376 & 497 & 41 & 1667 \\
\hline & & & $(28,6)$ & $(16,7)$ & $(22,5)$ & $(29,8)$ & $(2,4)$ & \\
\hline \multirow[t]{2}{*}{ WWeizen } & N100 & 148 & 463 & 556 & 376 & 908 & 41 & 2344 \\
\hline & & & $(19,7)$ & $(23,8)$ & $(16,0)$ & $(38,8)$ & $(1,7)$ & \\
\hline \multirow[t]{2}{*}{ WGerste } & N 50 & 76 & 364 & 286 & 359 & 511 & 22 & 1542 \\
\hline & & & $(23,6)$ & $(18,6)$ & $(23,3)$ & $(33,1)$ & $(1,4)$ & \\
\hline \multirow[t]{2}{*}{ WGerste } & N100 & 168 & 441 & 631 & 359 & 1020 & 22 & 2473 \\
\hline & & & $(17,8)$ & $(25,6)$ & $(14,5)$ & $(41,2)$ & $(0,9)$ & \\
\hline
\end{tabular}

$\mathrm{N}_{2} \mathrm{O}_{\text {Feld }}=$ in situ $\mathrm{N}_{2} \mathrm{O}$-Feldemission, $\mathrm{N}_{2} \mathrm{O}_{\text {ind }}=$ indirekte $\mathrm{N}_{2} \mathrm{O}$-Emission.

$\mathrm{KS}(\mathrm{D})=$ Emissionen aus Kraftstoff (Diesel), $\mathrm{D}=$ Emissionen aus Düngemittelbereitstellung, PS = Emission aus Pflanzenschutzmittelbereitstellung,

* = Summe der klimawirksamen Emissionen beim Anbau der jeweiligen Frucht.

Werte in Klammern ( ) geben den jeweiligen prozentualen Anteil an der Summe (Total) der $\mathrm{CO}_{2^{-}}$ Äquivalente an. 
Produktion von WWeizen und WGerste wies bei nahezu identischem NDüngungsniveau eine ähnlich hohe treibhauswirksame Gesamtemission auf. Gegenüber der Rapsproduktion ergab sich eine Reduktion der Treibhauswirksamkeit um rund $18 \%$ für die niedrige N-Düngung ( $\mathrm{N} 50$ ) bzw. um ca. $12 \%$ für die intensive Düngung (N100) (vgl. Tab. 3-11).

Der Anteil der direkten Feldemissionen an der gesamten Atmosphärenbelastung durch die Produktion der drei Fruchtarten schwankte zwischen 17,8 (WGerste, N100) und 39,2 \% (WRaps, N 50) (Tab. 3-11). Direkte und indirekte Feldemissionen als Quelle biologischen Ursprungs machen gemeinsam einen Anteil von 42,2 (WGerste, $N$ 50) bis maximal $54,2 \%$ (WRaps, N 50) an der gesamten Atmosphärenbelastung aus. Es zeigt sich also, daß es gerade die $\mathrm{N}_{2} \mathrm{O}$-Emissionen biologischen Ursprungs sind, die das Ausmaß der Atmosphärenbelastung bestimmen. Dieses steht im Widerspruch zur Annahme von KALTSCHMITT und REINHARD (1997), die der Auffassung sind, daß die biologisch bedingten $\mathrm{N}_{2} \mathrm{O}$ Emissionen im Hinblick auf eine Gesamtbilanzierung der treibhauswirksamen Emissionen, die mit der Nutzenergiebereitstellung aus Biomasse verbunden sind, eine untergeordnete Rolle spielen.

Die Klimawirksamkeit der Bereitstellung und des Verbrauchs von Dieselkraftstoff (Emissionen energetischen Ursprunges) fiel in den N-reduzierten Varianten (N 50) der Fruchtarten mit durchschnittlich 21 , in den intensiv gedüngten $\mathrm{N}$-Varianten (N100) im Mittel mit 14\% der Gesamtemissionen ins Gewicht (Tab. 3-11). Der chemische Pflanzenschutz spielte in allen Fruchtarten (N 50 und N100) mit einem Anteil von weniger als $2,5 \%$ an der totalen Atmosphärenbelastung eine untergeordnete Rolle. In allen Varianten der Rotation trugen die energetisch bedingten Bereitstellungsemissionen des applizierten N-Düngers (beide N-Varianten) mit 27 - $41 \%$ stark zur Totalbelastung der Atmosphäre bei.

Eine Verringerung der N-Düngung beim WRaps führte zu einem Rückgang der Gesamtemissionen (Total) um 28, im WWeizen um 29 und in der WGerste um $38 \%$, obwohl die direkten $\mathrm{N}_{2} \mathrm{O}$-Emissionen aus dem Felde nahezu gleich blieben. Die Reduktion der Totalemissionen war auf dem Versuchsstandort nur auf einen Rückgang der energetisch bedingten Bereitstellungsemissionen des applizierten Stickstoffs sowie auf einen Rückgang der biologisch bedingten indirekten $\mathrm{N}_{2} \mathrm{O}$ Emissionen $(=0,75 \%$ des applizierten $\mathrm{N})$ zurückzuführen.

Unterstellt man die energetische Verwertung des WRapses, so müssen für eine Lebenswegbilanz auch noch die klimarelevanten Emissionen bei der Weiterverarbeitung des Erntegutes sowie die $\mathrm{N}_{2} \mathrm{O}$ - und $\mathrm{CH}_{4}$-Emissionen bei der Verbrennung des Rapsmethylesters (RME) berücksichtigt werden. 
Auf dem Reinshof wurden im Versuchsmittel jährlich $32 \mathrm{dt} \mathrm{ha}^{-1}$ WRaps-TM geerntet, was ca. $1200 \mathrm{~kg} \mathrm{RME} \mathrm{ha-1} \mathrm{a}^{-1}$ entspricht. Bei einem Heizwert von 37,2 MJ/kg entspricht dies dem Energieertrag von $44640 \mathrm{MJ} \mathrm{ha}^{-1} \mathrm{a}^{-1}$. Da sowohl für die Rapsproduktion als auch für die nachfolgende RME-Herstellung Energie eingesetzt wird, ist der Nettoenergieertrag deutlich geringer. Insgesamt betragen die Energieaufwendungen von der Pflanzenproduktion bis zur Ölgewinnung rund $15000 \mathrm{MJ} \mathrm{ha}^{-1} \mathrm{a}^{-1}$ (KALTSCHMITT und REINHARD, 1997). Für die anschließende Raffination und Umesterung sind nochmals rund $8000 \mathrm{MJ} \mathrm{ha}^{-1} \mathrm{a}^{-1}$ nötig. Neben dem RME werden in dieser Produktionskette auch noch andere Produkte mit ökonomischem Wert, wie z.B. Rapskuchen und Glycerin (= Kuppelprodukte), erzeugt. Sowohl die bei der Produktion aufgewendete Energie als auch die auftretenden Umweltbelastungen können daher nicht ausschließlich der RMEProduktion angelastet werden. Verteilt man die Energieaufwendungen und Umweltbelastungen entsprechend der Energiegehalte der erzeugten Kuppelprodukte (KALTSCHMITT und REINHARD, 1997), so entfallen auf den RME ca. $60 \%$ der Aufwendungen und Umweltwirkungen, die im Zuge des Anbaus von Winterraps und der Ölgewinnung entstehen. Bei der ausschließlichen Prozeßkette der RMEHerstellung sind es ca. $96 \%$. Der Netto-Energieertrag der RME-Produktion würde demnach rund $30000 \mathrm{MJ} \mathrm{ha}^{-1} \mathrm{a}^{-1}$ betragen (KALTSCHMITT und REINHARD, 1997). Die Nutzung einer entsprechenden Menge an Dieselkraftstoff würde die Atmosphäre mit ca. 2,5 t $\mathrm{CO}_{2}$-Äquivalenten belasten. Dieses $\mathrm{CO}_{2}$-Einsparungspotential der Substitution von Dieselkraftstoff durch RME wird durch die Emission von Treibhausgasen (Tab. 3-11) im Rahmen der Rapsproduktion reduziert. Folgt man dem Bewertungsschema der Kuppelprodukte von KALTSCHMITT und REINHARD (1997), so sind $60 \%$ dieser Emissionen aus der Rapsproduktion dem RME anzurechnen. Die $\mathrm{N}_{2} \mathrm{O}$-Emissionen biologischen Ursprungs $\left(\mathrm{N}_{2} \mathrm{O}_{\text {Feld }}+\mathrm{N}_{2} \mathrm{O}_{\text {ind }}\right)$ der praxisüblich gedüngten Rapsvariante (N100) würden demnach auf den Versuchsflächen des Reinshofes mit anzurechnenden $\mathrm{CO}_{2}$-Äquivalenten in Höhe von $835 \mathrm{~kg} \mathrm{ha}^{-1} \mathrm{a}^{-1}$ das $\mathrm{CO}_{2}$-Eisparungspotential $\left(2500 \mathrm{~kg} \mathrm{CO}_{2} \mathrm{ha}^{-1} \mathrm{a}^{-1}\right)$ bereits um ein Drittel reduzieren. Die Emissionen energetischen Ursprungs (Tab. 3-11) kämen mit ca. $800 \mathrm{~kg} \mathrm{CO}_{2}$ ha $^{-1} \mathrm{a}^{-1}$ hinzu, so daß die gesamte Atmosphärenbelastung durch den Anbau von WRaps schon $66 \%$ des Einsparungspotentials zunichte macht.

Berücksichtigt werden müssen neben den klimawirksamen Emissionen beim Anbau von Winterraps (Tab. 3-11) noch die treibhauswirksamen Emissionen der Prozeßkette der nachfolgenden RME-Produktion sowie der RME-Verbrennung $\left(\mathrm{N}_{2} \mathrm{O}\right.$ und $\mathrm{CH}_{4}$-Emissionen). Hierdurch würde das Einsparungspotential nochmals um ca. $900 \mathrm{~kg} \mathrm{CO}_{2} \mathrm{ha}^{-1} \mathrm{a}^{-1}$ reduziert (KALTSCHMITT und REINHARD, 1997). Die $2500 \mathrm{~kg}$ $\mathrm{CO}_{2} \mathrm{ha}^{-1} \mathrm{a}^{-1}$, die bei der Substitution von Dieselkraftstoff durch RME zunächst eingespart werden, müssen bei Betrachtung des gesamten Lebensweges der RME- 
Produktion durch zusätzliche klimarelevante Emissionen in Höhe von ca. $2535 \mathrm{~kg}$ $\mathrm{CO}_{2} \mathrm{ha}^{-1} \mathrm{a}^{-1}$ belastet werden. Damit wäre das gesamte $\mathrm{CO}_{2}$-Einsparungspotential der Produktion von Rapsölmethylester vollständig zunichte gemacht. Zu einem ähnlichen Ergebnis kamen WOLFENSBERGER und DINKEL (1997) in ihrer ökologischen Beurteilung nachwachsender Rohstoffe. Die Lebenswegbilanz des RME zeigt, daß trotz des sehr niedrigen $\mathrm{N}_{2} \mathrm{O}$-Emissionsniveaus auf dem Standort „Reinshof“ die biologischen Lachgasemissionen eine bestimmende Größe der $\mathrm{CO}_{2}$-Bilanz sind. 


\section{Untersuchungen über die $\mathrm{N}_{2}$ O- und $\mathrm{N}_{2}$-Emissionen in unter- schiedlichen Tiefen eines Gley-Auenbodens im südnieder- sächsischen Leinetal}

\subsection{Einleitung}

Aus Böden emittiertes $\mathrm{N}_{2} \mathrm{O}$ kann sowohl nitrifikativer als auch denitrifikativer Herkunft sein (FIRESTONE et al., 1979). Die Nitrifikation findet i.d.R. nach BouwmaN (1990) nahe der Oberfläche unter aeroben Bedingungen statt. FIRESTONE und DAVIDSON (1989) geben an, daß dabei die $\mathrm{N}_{2} \mathrm{O}$-Freisetzung im allgemeinen deutlich weniger als $1 \%$ der nitrifizierten Stickstoffmenge ausmacht.

Die Denitrifikation hingegen findet nach FIRESTONE und DAVIDSON (1989) überwiegend in tieferen Bodenhorizonten unter anaeroben Bedingungen statt und kann bei hohem Wassergehalt des Bodens stark schwanken. Im Oberboden können denitrifikative $\mathrm{N}$-Verluste durch Verringerung der $\mathrm{O}_{2}$-Diffusion in den Boden hinein z.B. bei hohem Bodenwassergehalt oder bei Bodenverdichtung eintreten. Zusätzlich können sie auch durch mikrobiellen Sauerstoffverbrauch infolge der Zersetzung leicht abbaubarer organischer Substanz (z.B. Erntereste mit engem C/N-Verhältnis) gefördert werden (FLESSA und BEESE, 1995). Nach WEIER et al. (1993a) bestimmt die Kohlenstoffverfügbarkeit des Bodens maßgeblich das $\mathrm{N}_{2} \mathrm{O} / \mathrm{N}_{2}$-Verhältnis. In größerer Bodentiefe kann es auch bei geringer Kohlenstoffverfügbarkeit infolge autotropher Reduktionsprozesse zu hohen gasförmigen N-Verlusten kommen (RoLLAND, 1995). AULAKH et al. (1992) berichten, daß der relative Anteil des Lachgases an den gesamten gasförmigen $\mathrm{N}-$ Verlusten denitrifikativer Herkunft zwischen 0 und $100 \%$ variieren kann. BECKER (1991) nennt die Wurzelzone als wichtigsten Ort der Denitrifikation, im nicht durchwurzelten Bereich der Dränzone sei hingegen nur von geringen Nitratabbauraten auszugehen.

In Untersuchungen über den N-Haushalt im System Pflanze-Boden bei Extensivierung einer Rapsfruchtfolge stellte LICKFETT (1993) auf dem in dieser Arbeit untersuchten Gley-Auenboden des Reinshof ein beträchtliches Defizit in N-Bilanzen fest. Er beobachtete, daß es mit zunehmender Bodentiefe selbst unterhalb des Wurzelraumes $(>100 \mathrm{~cm}$ ) zu abnehmenden Nitratgehalten im Bodenwasser kam. Er vermutete aufgrund weiterer Untersuchungen, daß dieses $\mathrm{N}$-Defizit durch gasförmige $\mathrm{N}$-Verluste verursacht wurde. Versuche zu ihrer Quantifizierung und zur ursächlichen Diagnose wurden nicht durchgeführt.

Die in Kapitel 3 der vorliegenden Arbeit dargestellten Ergebnisse zeigen u.a., daß es auf diesem Gley-Auenboden beim Anbau der geprüften Fruchtarten zu 
vergleichsweise geringeren $\mathrm{N}_{2} \mathrm{O}$-Jahresemissionen kam als z.B. auf den Versuchsstandorten Scheyern und Canstein (vgl. FLESSA et al., 1998).

Die Befunde von LICKFETT (1993) wie auch die eigenen Ergebnisse aus den Feldversuchen gaben Anlaß, die Stickstoffmetabolik $\left(\mathrm{N}_{2} \mathrm{O}, \mathrm{N}_{2}\right)$ im Profil dieses Bodens zu untersuchen. Hierzu wurden Laboruntersuchungen unter Einsatz der von HANTSCHEL et al. (1994) beschriebenen Mikrokosmenanlage durchgeführt. Unter streng kontrollierten Bedingungen wurde an ungestörten Bodensäulen des Ap-, Ahund Go-Gr-Horizontes dieses Bodens die Lokalisation der $\mathrm{N}_{2} \mathrm{O}-$ Genese sowie die Denitrifikationsleistung in den Horizonten mit Hilfe der ${ }^{15} \mathrm{~N}$-Gasfreisetzungsmethode untersucht. 


\subsection{Material und Methoden}

\subsubsection{Entnahme von ungestörten Bodensäulen}

Für die Mikrokosmenversuche wurden dem Versuchsglied R-W-G des Versuchsstandortes Reinshof ungestörte Bodensäulen entnommen (vgl. Tab. 3-1). In den Tiefen 5 - 30 (Ap), 30 - 55 (Ah) und 140 - $165 \mathrm{~cm}$ (Go-Gr) wurden Plexiglaszylinder unter ständiger Freipräparation des seitlich anstehenden Bodens vorsichtig $25 \mathrm{~cm}$ tief in den Boden eingeschlagen. Gleichzeitig wurden aus den betreffenden Tiefen Bodenproben zur Bestimmung der physikalischen und chemischen Eigenschaften (vgl. Kap. 3.2.3.4 und Kap. 3.2.3.5) entnommen. Anschließend wurden die Bodensäulen in die Mikrokosmenanlage integriert.

\subsubsection{Funktion und Betrieb der Mikrokosmenanlage}

In der Mikrokosmenanlage können maximal 64 Bodensäulen bei variabler Temperatur inkubiert werden, für den hier durchgeführten Versuchsansatz wurde eine konstante Temperatur von $10{ }^{\circ} \mathrm{C}$ eingestellt. Der Gasfluß über die Bodensäulen sowie die Beregnung wurden über die Turbo Pascal Software MK 2 (Fa. UFE, Göttingen) gesteuert. HANTSCHEL et al. (1994) beschrieben die Anlage detailliert.

Nachdem die ungestörten Bodensäulen (Höhe $25 \mathrm{~cm}$, Durchmesser 14,6 cm, Oberfläche 162,8 $\mathrm{cm}^{2}$ ) am unteren Ende der Plexiglaszylinder (Höhe $30 \mathrm{~cm}$ ) bündig präpariert waren, wurden die gesamten Säulen mit der Bodenplatte und dem Deckel luftdicht verschlossen (Abb. 4-1). Über der Bodensäule befand sich ein Luftraum („Headspace“) mit einem Volumen von ca. 0,8 I. Dieser wurde ständig mit Außenluft durchspült, wobei eine konstante Flußrate von ca. $14 \mathrm{ml} \mathrm{min}^{-1}$ eingestellt wurde. Die Entnahme einer Probe für die Spurengasanalyse (vgl. Kap. 3.2.3.2) erfolgte manuell mit zuvor evakuierten Probenflaschen direkt aus dem Gasstrom der Luftabfuhr an 2 bis 3 Terminen pro Woche. Um die Gasemissionen der Bodensäulen berechnen zu können, wurden pro Termin in gleicher Weise insgesamt 4 Referenzproben aus der zuströmenden Frischluft gezogen. 


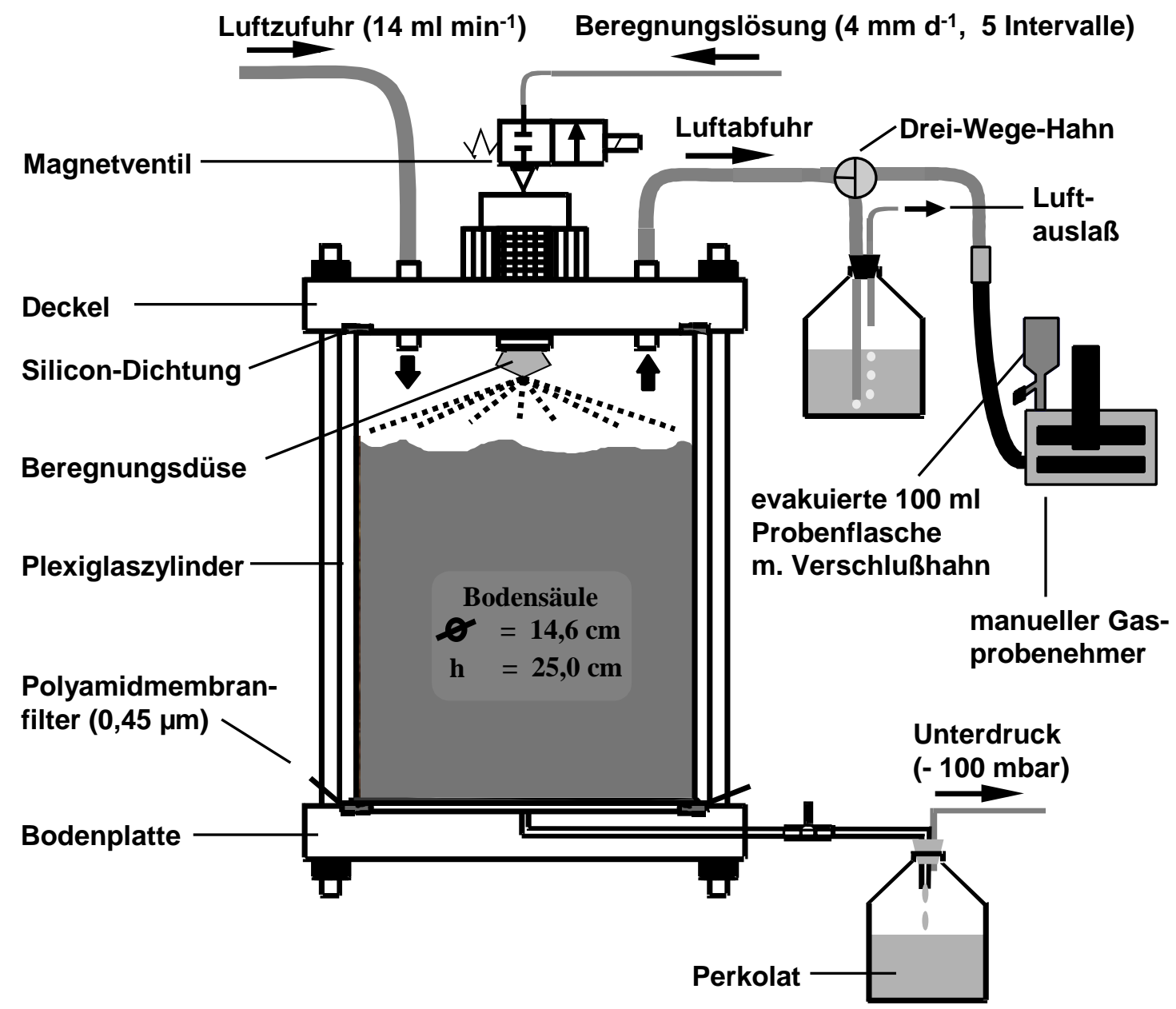

Abb. 4-1: $\quad$ Mikrokosmenanlage

Die Beregnungslösungen wurden über eine Düse im Deckel mit einer Rate von $4 \mathrm{~mm}$ $\mathrm{d}^{-1}$ in 5 Intervallen (entspr. 65,12 ml) zugeführt (vgl. Abb. 4-1). Am unteren Ende der Säulen war unterhalb eines Polyamidfilters (Porengröße 0,45 $\mu \mathrm{m}$, Fa. Sartorius) ein Unterdruck von -100 mbar - entsprechend einer Wasserspannung von $\mathrm{pF}=2$ angelegt. Dieses gewährleistete eine ständige Abfuhr der Perkolate aus den Bodensäulen. Die im Polyamidfilter filtrierten Perkolate wurden in Glasflaschen gesammelt, ein- bis zweimal wöchentlich entnommen, quantifiziert und bis zur Analyse auf $\mathrm{NO}_{3}-\mathrm{N}$ - und $\mathrm{NH}_{4}-\mathrm{N}$ tiefgefroren (vgl. Kap. 3.2.3.5.4).

In der Konditionierungsphase (Versuchsbeginn) wurden die Bodensäulen über einen Zeitraum von 55 Tagen mit einer 0,01 $\mathrm{M} \mathrm{CaSO}_{4}$-Lösung $\left(4 \mathrm{~mm} \mathrm{~d}^{-1}\right)$ beregnet. Während dieser Zeit wurden etwa zwei volumetrische Wassergehalte einer jeden Säule über die Perkolation des Sickerwassers ausgetauscht. Zum einen sollte sich während der Konditionierungsphase in den Säulen ein hydraulisches Gleichgewicht einstellen, zum anderen sollte das bei der Entnahme der Säulen im Boden 
befindliche Nitrat sowie andere akkumulierte gelöste Stoffe ausgewaschen werden. Außerdem bot sich die Möglichkeit, gegen Ende der Konditionierungsphase bei konstanten N-Austrägen über die Perkolate eine Aussage über die Mineralisationsrate des Bodens zu treffen.

\subsubsection{Versuchsvarianten}

Die Bodensäulen entstammten dem Ap-, Ah-, sowie dem Go-Gr-Horizont (Tab. 4-1) des Versuchsstandortes (vgl. Kap. 4.2.1). Die Bodensäulen wurden in 4-facher Wiederholung kontinuierlich mit Nitratlösungen unterschiedlicher Konzentrationen sowie im Laufe des Versuches auch mit Ammonium- bzw. ${ }^{15} \mathrm{~N}$-markiertem Nitrat beregnet. Zur Vereinfachung fand eine Aufteilung des gesamten Versuchsablaufes in zwei Phasen statt:

Tab. 4-1: Versuchsvarianten der Modellversuche

\begin{tabular}{|c|c|c|c|c|c|c|}
\hline \multirow[b]{3}{*}{$\begin{array}{l}\text { Tiefe u. GOF } \\
\text { [cm] }\end{array}$} & \multirow[b]{3}{*}{ Horizont } & \multirow[b]{3}{*}{$\mathbf{n}$} & \multicolumn{4}{|c|}{ Beregnungslösung } \\
\hline & & & \multicolumn{2}{|c|}{ Phase I } & \multicolumn{2}{|c|}{ Phase II } \\
\hline & & & N-Form & $\begin{array}{c}\text { N-Konzentration } \\
\text { [ppm] }\end{array}$ & N-Form & $\begin{array}{c}\text { N-Konzentration } \\
\text { [ppm] }\end{array}$ \\
\hline $5-30$ & Ap & 4 & $\mathrm{NO}_{3}^{-}$ & 100 & $\mathrm{NH}_{4}^{+}$ & 100 \\
\hline $30-55$ & Ah & 4 & $\mathrm{NO}_{3}^{-}$ & 100 & $\mathrm{NH}_{4}^{+}$ & 100 \\
\hline $30-55$ & Ah & 4 & $\mathrm{NO}_{3}^{-}$ & 50 & ${ }^{15} \mathrm{NO}_{3}^{-}$ & 50 \\
\hline $140-165$ & Go-Gr & 4 & $\mathrm{NO}_{3}^{-}$ & 25 & ${ }^{15} \mathrm{NO}_{3}^{-}$ & 25 \\
\hline
\end{tabular}

In Phase I wurden die Bodensäulen des Ap- und des Ah-Horizontes kontinuierlich mit einer 100 ppm-Nitrat-N-Lösung $\left(\mathrm{Ca}\left(\mathrm{NO}_{3}\right)_{2}\right)$ beregnet (Tab. 4-1). Der für die Beregnungslösung genutzte Konzentrationsbereich war so ausgewählt, wie er auch in der Bodenlösung eines landwirtschaftlich genutzten Oberbodens zu finden ist. Bei $4 \mathrm{~mm}$ täglicher Beregnungsmenge entsprach dies einer Stickstoffzufuhr in Höhe von $4 \mathrm{~kg} \mathrm{~N} \mathrm{ha}^{-1} \mathrm{~d}^{-1}$. Eine zweite Säulenvariante aus dem Ah-Horizont wurde mit einer 50 ppm-Nitrat-N-Lösung entsprechend $2 \mathrm{~kg} \mathrm{~N} \mathrm{ha}^{-1} \mathrm{~d}^{-1}$ beregnet, während dem GoGr-Horizont über eine 25 ppm-NO ${ }_{3}-\mathrm{N}$-Lösung $1 \mathrm{~kg} \mathrm{~N} \mathrm{ha}^{-1} \mathrm{~d}^{-1}$ zugeführt wurde. Grund für eine geringere Nitratzufuhr bei den Bodensäulen aus tieferen Bodenhorizonten ist, daß es auch in den Bodenlösungen landwirtschaftlich genutzter Böden mit zunehmender Bodentiefe zu abnehmenden Nitratgehalten kommt.

In Phase II wurden der Ap- und der Ah-Horizont anstatt der 100 ppm Nitrat-N-Lösung mit einer Ammoniumlösung $\left(\left(\mathrm{NH}_{4}\right)_{2} \mathrm{SO}_{4}\right)$ gleicher $\mathrm{N}$-Konzentration beregnet (vgl. Tab. 


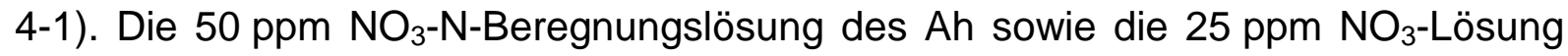
des Go-Gr wurden in Phase II durch ${ }^{15} \mathrm{~N}$-markierte Nitratlösungen $\mathrm{Ca}\left(\mathrm{NO}_{3}\right)_{2}$ gleicher Stickstoffkonzentration ersetzt (Ap-Horizont: 95\%-ige, Go-Gr-Horizont $50 \%$-ige ${ }^{15} \mathrm{~N}$ Anreicherung).

\subsubsection{Berechnung der Spurengasflüsse in den Mikrokosmen}

Die Flußrate $\left(\mathrm{F}_{\mathrm{Gas}}\right)$ von $\mathrm{CO}_{2}$ und $\mathrm{N}_{2} \mathrm{O}$ pro Flächeneinheit wird nach der folgenden Gleichung berechnet:

$$
\begin{aligned}
& \mathrm{F}_{\mathrm{Gas}}=\left(\frac{\mathrm{dc}^{*} \mathrm{Mi}^{*} \mathrm{Gr}}{\mathrm{Mv}^{*} \mathrm{~A}}\right) * 60 * 10^{-3 *} R \quad \mathrm{CO}_{2}:\left[\mathrm{mg} \mathrm{m}^{-2} \mathrm{~h}^{-1}\right] \\
& \mathrm{N}_{2} \mathrm{O}:\left[\mu \mathrm{g} \mathrm{m}^{-2} \mathrm{~h}^{-1}\right]
\end{aligned}
$$

$\mathrm{dc}=$ Konzentrationsänderung von $\mathrm{CO}_{2}$ [ppmv] sowie von $\mathrm{N}_{2} \mathrm{O}$ [ppbv] zwischen zugeführter und abgeführter Luft

$\mathrm{Mi}=$ Molmasse $\left[\mathrm{g} \mathrm{mol}{ }^{-1}\right]$

$\mathrm{Gr}=$ Gasfluß $\left[\mathrm{l} \mathrm{h}{ }^{-1}\right]$

$\mathrm{Mv}=$ Molvolumen $\left[\mathrm{mol}^{-1}\right]$

$A=$ Bodenoberfläche der Bodensäule $\left[\mathrm{m}^{2}\right]$

$\mathrm{R}=$ Reduktionsfaktor (vgl. Kap. 3.1.3.3)

Die Konzentrationsänderung der Gase ergibt sich aus der Differenz zwischen zugeführter Luft und abgeführter Luft.

\subsection{5 pF-Charakteristik der Bodenhorizonte}

Parallel zu der unter 4.2.1 beschriebenen Entnahme der Bodensäulen wurden aus den Ap-, Ah- und Go-Gr-Bodenhorizonten Stechzylinderproben $\left(100 \mathrm{~cm}^{3}, \mathrm{n}=8\right)$ entnommen. Mit Hilfe der Unterdruckmethode (HARTGE, 1992) wurden die pF-Kurven aufgenommen (Abb. 4-2). Diese geben an, welches Potential (negativer Druck, Spannung) bei verschiedenen Wassergehalten im Boden vorliegen. 


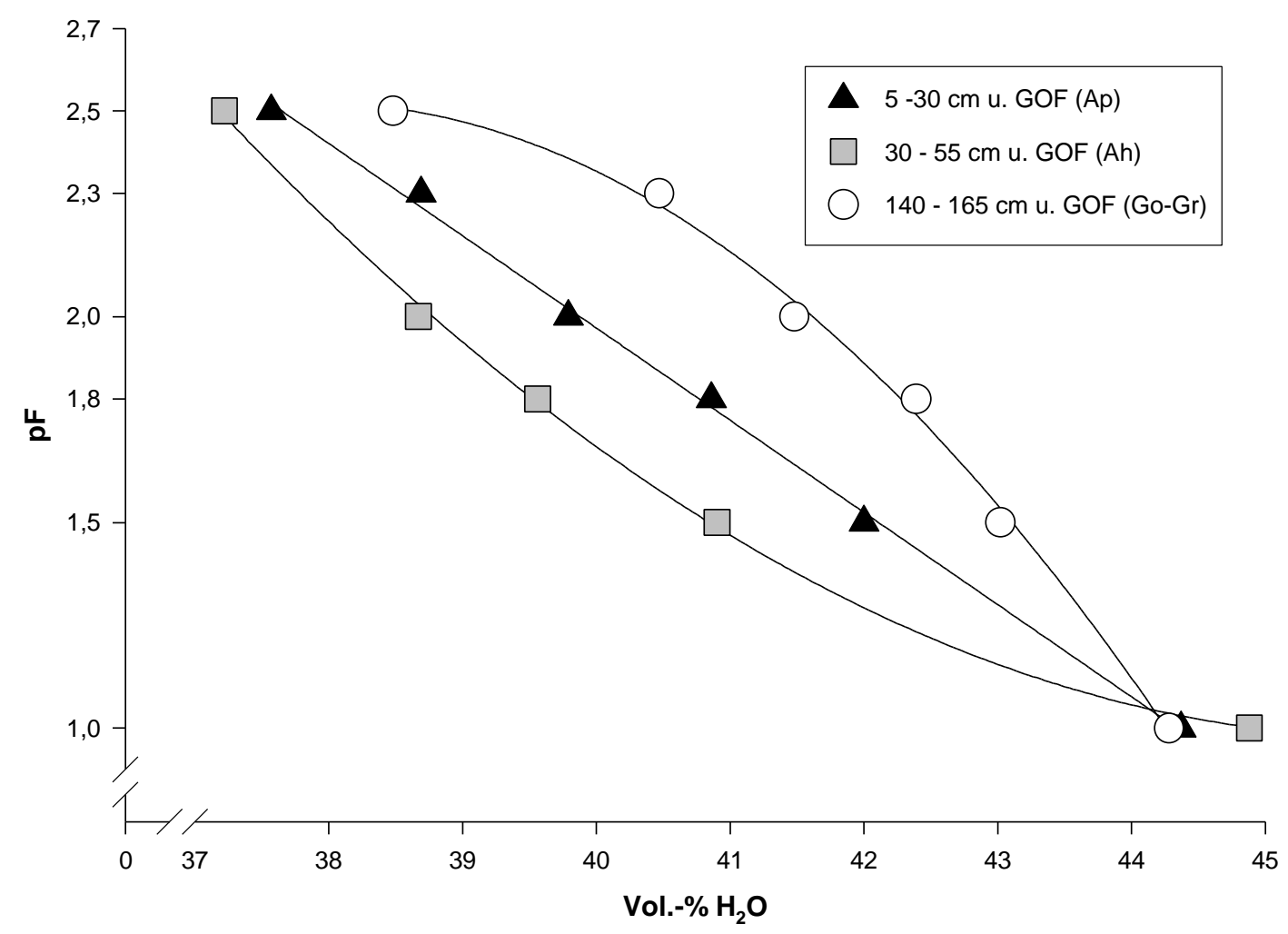

Abb. 4-2: $\quad$ pF-Kurven des Ap-, Ah- und Go-Gr-Horizontes $(\mathrm{n}=8)$

\subsubsection{Physikalische Eigenschaften der Bodensäulen}

Die mittleren Lagerungsdichten, die mittleren volumetrischen Wasser- und Luftgehalte (Vol.H20 und Vol.Luft) sowie der prozentuale Anteil der Vol.H20 am Gesamtporenvolumen (WFPS) der Bodensäulen des Ap-, Ah- und Go-GrBodenhorizontes zu Versuchsende nach Beregnung mit $4 \mathrm{~mm} \mathrm{~d}^{-1}$ sind in Tabelle 4-2 dargestellt. Die Lagerungsdichte der aus dem Ap-Horizont und Ah-Horizont entnommenen Bodensäulen stimmte nach Versuchsende mit den über 2 Jahre regelmäßig bestimmten Lagerungsdichten unter Feldbedingungen in 5-10 cm (Ap) und 30-35 cm (Ah) weitgehend überein (vgl. Tab. 3-1). Die Lagerungsdichte der Bodensäule aus 140-165 cm unter GOF (Go-Gr-Horizont) lag mit $1,25 \mathrm{~g} \mathrm{~cm}^{-3}$ nach Versuchsende unter der im April 1996 ermittelten natürlichen Lagerungsdichte von $1,5 \mathrm{~g} \mathrm{~cm}^{-3}$ in $145-150 \mathrm{~cm}$ unter GOF. Dieses weist auf eine inhomogene Beschaffenheit des Go-Gr-Horizontes mit unterschiedlicher Lagerungsdichte hin.

Nach kontinuierlicher Beregnung während der Versuchsdurchführung (4 $\left.\mathrm{mm} \mathrm{d}^{-1}\right)$ wies der Ap-Horizont ein wassergefülltes Porenvolumen von $82 \%$ auf, die mittlere Saugspannung lag im Bereich pF 1,7 (Tab. 4-2, vgl. Abb. 4-2). Bei den Bodensäulen aus dem Ah-Horizont lag das WFPS bei $77 \%$ und die Saugspannung im Bereich von 
pF 2,0. Die Säulen des Go-Gr-Horizontes wiesen hingegen ein WFPS von $93 \%$ bei nur noch ca. 4 \% Luftgehalt auf; die Saugspannung dieser Bodensäulen lag bei pF < 1 (in Abb. 4-1 nicht mehr dargestellter Bereich). Die Bodenfeuchte der Säulen des Ap- wie auch des Ah-Horizontes bewegten sich unter Versuchsbedingungen also im Bereich der Feldkapazität, während bei der Variante aus dem Go-Gr-Horizont ein gesättigter Wasserfluß vorliegt.

Tab. 4-2: $\quad$ Bodenphysikalische Eigenschaften der Bodensäulen nach Beregnung mit $4 \mathrm{~mm} \mathrm{~d}^{-1}$ (nach Versuchsende ermittelt).

\begin{tabular}{|c|c|c|c|c|c|c|c|}
\hline $\begin{array}{l}\text { Tiefe } \\
\text { u. GOF } \\
{[\mathrm{cm}]}\end{array}$ & $\begin{array}{l}\text { Boden- } \\
\text { horizont }\end{array}$ & $n$ & $\begin{array}{l}\text { Lagerungs- } \\
\text { dichte } \\
\left(d_{B}\right) \\
{\left[g ~ c m^{-3}\right]}\end{array}$ & $\begin{array}{c}\text { Volumetrischer } \\
\text { Wassergehalt } \\
\text { (Vol..H2O) } \\
\text { [\%] }\end{array}$ & $\begin{array}{l}\text { Volumetrischer } \\
\text { Luftgehalt } \\
\text { (Vol.-Luft) } \\
\text { [\%] }\end{array}$ & WFPS & $\begin{array}{c}\text { Saug- } \\
\text { spannung } \\
{[\mathrm{pF}]}\end{array}$ \\
\hline $5-30$ & Ap & 4 & 1,30 & 41,5 & 9,2 & 82,4 & $\sim 1,7$ \\
\hline $30-55$ & Ah & 8 & 1,32 & 38,5 & 12,0 & 76,7 & $\sim 2,0$ \\
\hline $140-165$ & Go-Gr & 4 & 1,25 & 49,0 & 3,8 & 93,0 & $<1,0$ \\
\hline
\end{tabular}

In Tabelle 4-3 sind die täglichen Beregnungsmengen $\left(B L_{d}\right)$ sowie die Porenwasservolumen (PV), wie sie sich unter den Versuchsbedingungen in den Bodensäulen einstellten, angegeben. Unterstellt man homogene Fließbedingungen sowie den Austausch des gesamten Bodenwassers einschließlich des Totwasseranteils durch die Beregnungslösung, so errechnet sich für den Ah-Horizont eine Zeit von 24 Tagen, für den Ap- von 26 Tagen und für den Go-Gr-Horizont von 30 Tagen für den Austausch von einem PV durch die $\mathrm{BL}_{d}$.

Tab. 4-3: $\quad$ Erforderliche Tage für den Austausch von einem Porenwasservolumen (PV) durch die täglichen Beregnungsvolumina $\left(\mathrm{BL}_{\mathrm{d}}\right)$ in den Bodensäulen.

\begin{tabular}{ccc|c|cc}
\hline $\begin{array}{c}\text { Tiefe u. } \\
\text { GOF } \\
\text { [cm] }\end{array}$ & $\begin{array}{c}\text { Boden- } \\
\text { horizont }\end{array}$ & $\mathbf{n}$ & $\mathbf{B L}_{\mathbf{d}}$ & $\mathbf{P V}$ & $\begin{array}{c}\text { Tage zum Austausch } \\
\text { von einem PV } \\
\text { [d] }\end{array}$ \\
\hline $5-30$ & Ap & 4 & 65,1 & [ml] & 25,9 \\
\hline $30-55$ & Ah & 8 & 65,1 & 1684 & 24,1 \\
\hline $140-165$ & Go-Gr & 4 & 65,1 & 1567 & 30,3 \\
\hline
\end{tabular}




\subsubsection{Bestimmung der Denitrifikation mit Hilfe der ${ }^{15} \mathrm{~N}$-Gasfreisetzungs- Methode (GFM)}

\subsubsection{Das Inkubationssystem}

Zur direkten Bestimmung der denitrifikativen $\mathrm{N}_{2}+\mathrm{NO}+\mathrm{N}_{2} \mathrm{O}$-Emissionen aus den Bodensäulen der Tiefen 30-55 cm und 140-165 cm unter GOF (vgl. Tab. 4-1) wurde in der Mikrokosmenanlage so lange ${ }^{15} \mathrm{~N}$-markiertes Nitrat (vgl. Kap. 4.2.3) verregnet, bis ca. 1,5 Porenwasservolumina (PV) durch die Perkolation der Lösung ausgetauscht waren. Anschließend wurde der Luftdurchfluß und die Beregnung beendet, die Beregnungsdüsen der Mikrokosmen durch ein Septum zur Gasprobenahme ersetzt sowie der Unterdruck am unteren Ende der Bodensäulen abgestellt (Abb. 4-3). Der Luftraum über den Bodensäulen (="Headspace") wurde

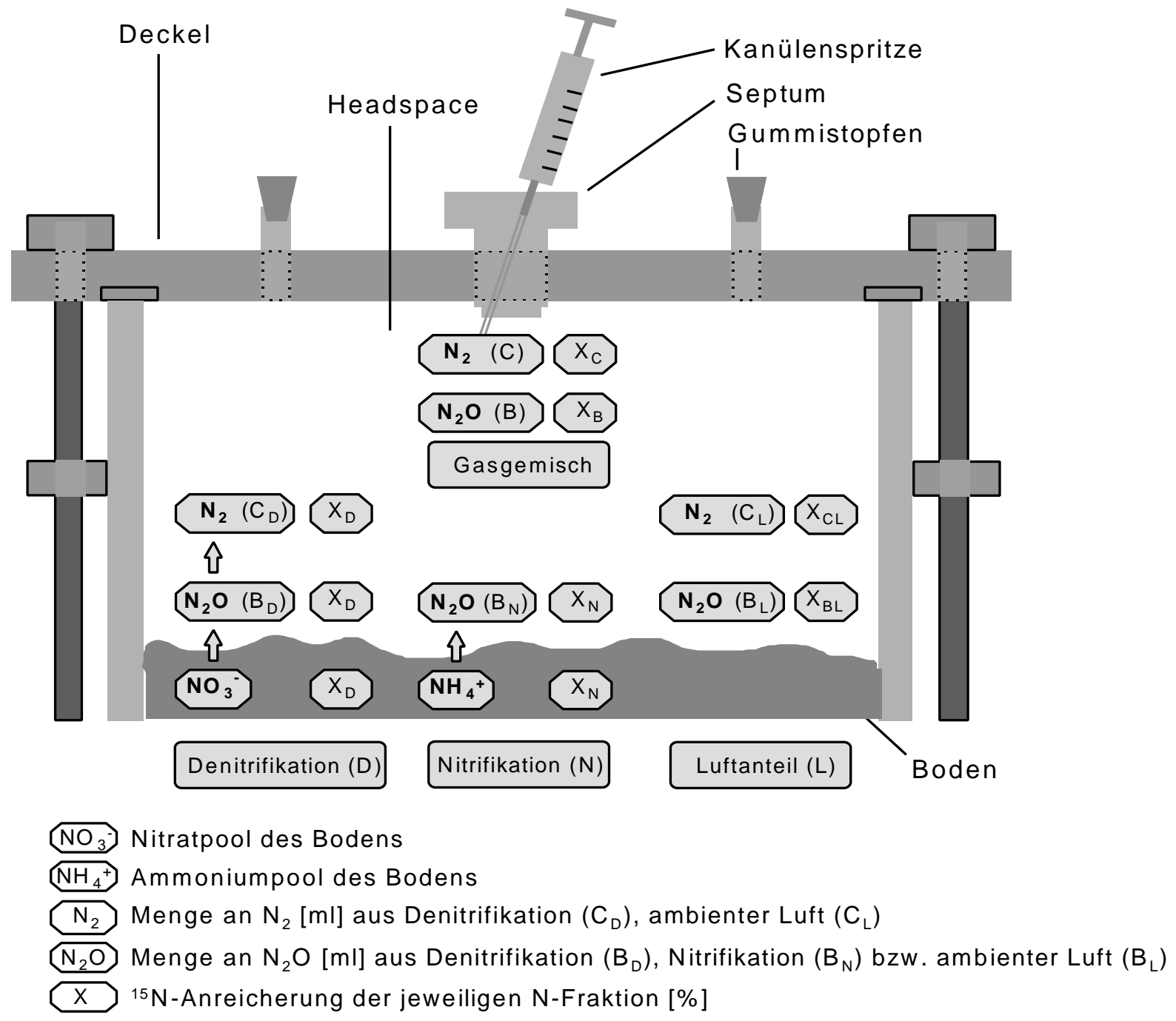

Abb. 4-3: $\quad$ Schematische Darstellung des Inkubationssystems zur direkten Bestimmung der denitrifikativen ${ }^{15} \mathrm{~N}$-Gasfreisetzung. 
mit atmosphärischer Luft gespült, um sicherzustellen, daß zu Inkubationsbeginn eine Konzentration von ca. 0,3663 Atom\% ${ }^{15} \mathrm{~N}\left(={ }^{15} \mathrm{~N}\right.$-Konzentration der atmosphärischen Luft) vorlag.

\subsubsection{Entnahme und Aufbereitung der Gasproben für die Isotopenanalyse}

4, 5 und 8 Tage nach Inkubationsbeginn erfolgten mittels PE-Spritzen Probenahmen von ca. $2 \mathrm{ml}$ Gas aus dem Headspace. Die aufgezogenen Spritzen wurden zur Abdichtung mit der Nadelspitze in Silikonstopfen gesteckt und innerhalb von 3 Stunden analysiert. Eine vor dem Abdichten der Mikrokosmen entnommene Gasprobe aus der Luftzufuhr des Systems ermöglichte die Bestimmung des ${ }^{15} \mathrm{~N}$ Referenzwertes der atmosphärischen Luft (vgl. Abb. 4-1).

\subsubsection{3 $\quad{ }^{15} \mathrm{~N}$ - Analytik}

Die ${ }^{15} \mathrm{~N}$-Gehalte der Gasproben (bis max. 1,5 Atom\% ${ }^{15} \mathrm{~N}$ ) wurden massenspektrometrisch (Fa. Finnigan, MAT 251) analysiert ${ }^{1}$. Das Gerät war mit einem C/NAnalysator (Fa. Carlo Erba, NA 1500) über ein Online-Split-Interface verbunden.

Nach Injektion von $1 \mathrm{ml}$ Probengas in den Trägergasstrom des Elementaranalysators erfolgt die Reduktion von $\mathrm{N}_{2} \mathrm{O}$ und $\mathrm{NO} z u \mathrm{~N}_{2}$ und die reduktive Eliminierung des störenden Sauerstoffs in einer Kupfersäule. Kohlendioxid wird in einem $\mathrm{CO}_{2}$-Filter entfernt.

Zur indirekten Bestimmung des denitrifikativen $\mathrm{N}_{2} \mathrm{O}$-Anteils in der Gasprobe $\left(\mathrm{N}_{2} \mathrm{O}+\mathrm{NO}+\mathrm{N}_{2}\right)$ wurde eine Parallelprobe über eine GC-Säule (Molsieb, $5 \AA$ ) geleitet, um $\mathrm{N}_{2} \mathrm{O}$ vor der Reduktion abzutrennen und somit später nur die NO- und $\mathrm{N}_{2}$ Fraktionen zu erfassen. Aus der Differenz der ${ }^{15} \mathrm{~N}$-Konzentrationen der parallelen Proben ließen sich somit die denitrifikativen ${ }^{15} \mathrm{~N}_{2} \mathrm{O}-\mathrm{N}$-Anteile errechnen. Die Stickstoffmonoxid-Anteile (NO) als ein Teil des verbleibenden ${ }^{15} \mathrm{~N}$-Anteils $\left(\mathrm{NO}+\mathrm{N}_{2}\right)$ wurden ebenfalls indirekt bestimmt, indem bei einer Parallelprobe eine NO-Falle vor den Elementaranalysator geschaltet wurde, um in dieser ausschließlich ${ }^{15} \mathrm{~N}_{2} \mathrm{zu}$ erfassen. Wie schon beim $\mathrm{N}_{2} \mathrm{O}$ konnte nun auch denitrifikatives ${ }^{15} \mathrm{NO}-\mathrm{N}$ aus der Differenz der ${ }^{15} \mathrm{~N}$-Konzentrationen der parallelen Proben bestimmt werden.

\footnotetext{
${ }^{1)}$ Die Isotopenanalyse der gasförmigen Proben wurde mit freundlicher Unterstützung von R. Langel im Isotopenlabor für biologische und medizinische Forschung der Universität Göttingen durchgeführt.
} 
Im Gasgemisch kommen die $\mathrm{N}_{2}$-Molmassen 28, 29 und 30 vor, jedoch werden nur die Massen $28\left({ }^{14} \mathrm{~N}^{14} \mathrm{~N}\right)$ und $29\left({ }^{14} \mathrm{~N}^{15} \mathrm{~N}\right)$ bestimmt. Wird ${ }^{15} \mathrm{~N}$-markierter, molekularer Stickstoff $\left(X_{D}\right.$, statistische ${ }^{15} \mathrm{~N}$-Verteilung), der durch Denitrifikation im Boden entstanden ist, mit dem molekularen Stickstoff der Luft (natürliche ${ }^{15} \mathrm{~N}$-Häufigkeit $\left(X_{C I}\right)$, statistische ${ }^{15} \mathrm{~N}$-Verteilung) vermischt, so ist das ${ }^{15} \mathrm{~N}_{2}$-Gemisch $\left(\mathrm{X}_{\mathrm{C}}\right)$ nichtstatistisch verteilt (Abb. 4-3). Deshalb würden bei der ausschließlichen Bestimmung des ${ }^{29} \mathrm{~N}_{2} /{ }^{28} \mathrm{~N}_{2}$ - Verhältnisses der ${ }^{30} \mathrm{~N}_{2}$-Anteil und somit die Denitrifikationsprozesse unterschätzt werden (HAUCK et al., 1958). Um dieses zu vermeiden, wurden parallel jeweils eine nicht equilibrierte und eine equilibrierte Gasprobe gemessen (MWE-Verfahren) (WELL et al., 1998). Mit Hilfe des Einsatzes von Mikrowellen wird in den Proben bei Unterdruck eine elektrodenlose Gasentladung erzeugt. Durch die dabei eintretende Dissoziation der $\mathrm{N}_{2}$-Moleküle und sich anschließende Neuverteilung der N-Atome wird eine statistische Verteilung der Molekülmassen erreicht, die bei der nicht equilibrierten Probe nicht gegeben ist. Somit kann die ${ }^{15} \mathrm{~N}$-Anreicherung der Gasproben fehlerfrei aus der Messung der Molekülmassen 28 und 29 errechnet werden. Die Messmethodik sowie das MWEVerfahren wurden von WELL (1993) ausführlich beschrieben. Eine direkte massenspektrometrische Bestimmung der Masse 30 war durch elektronisches Rauschen und durch die NO-Produktion (=Masse 30) in der lonenquelle nicht möglich.

Die Messung eines Standardgases erbrachte nach 6 Wiederholungen eine mittlere ${ }^{15} \mathrm{~N}$-Anreicherung von 0,3691 Atom-\%, der Variationskoeffizient der Wiederholungen lag bei 0,019 Atom-\%. Messwiederholungen der atmosphärischen Luft $(n=27)$ ergaben einen Variationskoeffizienten in Höhe von 0,034 \% (Tab. 4-4).

Tab. 4-4: Reproduzierbarkeit der ${ }^{15} \mathrm{~N}-$ Gasanalyse.

\begin{tabular}{lcccc}
\hline Gasprobe & $\overline{\mathbf{X}}$ [Atom-\%] & $\mathbf{n}$ & $\mathbf{s}$ & Vk [\%] \\
\hline Standard ${ }^{15} \mathrm{~N}$ & 0,3691 & 6 & 0,000069 & 0,019 \\
Luft $_{\text {atmosph. }}{ }^{15} \mathrm{~N}$ & 0,3666 & 27 & 0,000123 & 0,034 \\
\hline
\end{tabular}




\subsubsection{Berechnung der denitrifikativen $\mathrm{N}_{2}$-Freisetzung}

Die ${ }^{15} \mathrm{~N}$-Anreicherungen gasförmiger $\mathrm{N}$-Komponenten im nicht equilibrierten und im equilibrierten Zustand wurden analytisch bestimmt. Bei der Bewertung der denitrifikativen ${ }^{15} \mathrm{~N}_{2}$-Freisetzung wird davon ausgegangen, daß die ${ }^{15} \mathrm{~N}$-Anreicherung in der Gasphase $\left(C_{D}\right)\left(X_{D}\right)$ der ${ }^{15} \mathrm{~N}$-Anreicherung des Nitrates entspricht $\left(\mathrm{NO}_{3}\right)\left(\mathrm{X}_{\mathrm{D}}\right)$ (Abb. 4-3). Grundlage zur Erfassung des gesamten gasförmigen N-Flusses aus dem Boden ist deshalb die Kenntnis der Anreicherung des nach der ${ }^{15} \mathrm{NO}_{3}$-Applikation in der Bodensäule befindlichen Gesamtnitrats $\left(\mathrm{NO}_{3}\right)(\mathrm{XD})$. Die ${ }^{15} \mathrm{~N}$-Anreicherung im Gesamtnitrat der Bodensäulen kann aus der massenspektrometrischen Bestimmung des $\mathrm{N}_{2}$ von equilibrierten ( $\mathrm{X}_{\mathrm{Ceq}}$ ) und nicht equilibrierten Gasproben ( $\mathrm{X}_{\mathrm{Cneq}}$ ) und aus der Anreicherung im $\mathrm{N}_{2}$ der atmosphärischen Luft $\left(\mathrm{X}_{\mathrm{CL}}\right)$ berechnet werden (BRUMME und ADEN, 1995):

$X_{D}[\%] \approx 100-\left(\frac{X_{\text {Cneq }}-X_{C L}}{X_{\text {Ceq }}-X_{C L}}\right) * 100$

Bei Kenntnis der Anreicherung des Bodennitrats $\left(X_{D}\right)$ läßt sich die Konzentration des durch Denitrifikation gebildeten $\mathrm{N}_{2}\left(\mathrm{C}_{\mathrm{D}}\right)$ berechnen:

$\mathrm{C}_{\mathrm{D}}[\%]=\left(\frac{\mathrm{XCeq}-\mathrm{XCL}}{\mathrm{XD}}\right) * C_{L}$

Die Gaskonzentrationen $C_{D}$ und $C_{L}$ sind unbekannt, jedoch kann $C_{L}$ gleich 1 gesetzt werden, da es der gesamten $\mathrm{N}_{2}$-Gasmenge im Anreicherungsvolumen oberhalb des Bodens entspricht und der denitrifikative $\mathrm{N}_{2}$-Anteil (1- $\mathrm{C}_{\mathrm{L}}$ ) verschwindend gering ist.

Zur Berechnung der $\mathrm{N}_{2}$-Masse im Anreicherungsvolumen dient das allgemeine Gasgesetz:

$N_{2}$ gesamt $[g]=\left(\frac{P * V}{R * T}\right) * 0,7809 * M$

$\mathrm{P} \quad=\quad$ Luftdruck $[\mathrm{Pa}]$

$\mathrm{V} \quad=\quad$ Volumen des Anreicherungsraumes $\left[\mathrm{m}^{3}\right]$

$\mathrm{R}=$ allgemeine Gaskonstante $\left(=8,3144 \mathrm{~J} \mathrm{~K}^{-1} \mathrm{~mol}^{-1}\right)$ 
$\mathrm{T} \quad=\quad$ Temperatur $[\mathrm{K}]$

$\mathrm{M}=\quad$ Molmasse $\left[\mathrm{g} \mathrm{mol}^{-1}\right]$

Es wird von 78,09\% Gesamtstickstoff im Anreicherungsvolumen ausgegangen.

Die Menge an $\mathrm{N}_{2}$ aus der Denitrifikation im Anreicherungsvolumen errechnet sich nach:

$\mathrm{N} 2$ freigesetzt $=\mathrm{N} 2$ gesamt ${ }^{*} \mathrm{CD} \quad[\mathrm{g}]$

\subsubsection{Statistische Auswertung}

Die Datenkalkulationen und Berechnungen von Mittelwert, Standardabweichung und Variationskoeffizient wurden mit Excel 7.0 (Microsoft) durchgeführt. 


\subsection{Ergebnisse}

Vor Beginn der Versuche wurden die Bodensäulen mit einer 0,01 $\mathrm{M} \mathrm{CaSO}_{4}$-Lösung $\left(4 \mathrm{~mm} \mathrm{~d}^{-1}\right)$ konditioniert (Kap. 4.2), die sich anschließenden Untersuchungen umfaßten Phase I und Phase II.

In Phase I erfolgte eine ausschließliche Nitratberegnung aller Bodenhorizonte. Für die Nitratlösungen wurde ein Konzentrationsbereich gewählt, wie er auch in den Bodenlösungen der entsprechenden Horizonte unter Feldbedingungen anzutreffen ist. Damit ist auch die vorgenommene Reduzierung der Nitratkonzentration mit zunehmender Bodentiefe begründet. Es soll im folgenden geklärt werden, ob und in welchen Bodenhorizonten es nach Applikation von Nitrat zu $\mathrm{N}_{2} \mathrm{O}$-Emissionen kommt. Im Rahmen einer Quantifizierung der Stickstoffflüsse in den Bodensäulen wurden neben der zweimal wöchentlich durchgeführten Bestimmung der $\mathrm{N}_{2} \mathrm{O}$-Emissionen die Nitrat- und Ammoniumausträge mit den Perkolaten einmal, in Zeiten stärkerer Dynamik auch zweimal wöchentlich bestimmt (Kap. 4.2). Phase I erstreckte sich über einen Zeitraum von 58 Tagen (Ap und Go-Gr-Horizont) bzw. über 62 Tage (AhHorizont).

Phase II unterschied sich zunächst nicht von der analytischen Vorgehensweise in Phase I. Um den Anteil nitrifikationsbürtiger $\mathrm{N}_{2} \mathrm{O}$-Emissionen vom ausgebrachten $\mathrm{N}$ Stickstoff abschätzen zu können, wurde der Ap- und der Ah-Horizont bei unveränderter Stickstoffzufuhr über einen Zeitraum von 60 (Ap) bzw. 56 Tagen (Ah) mit Ammonium beregnet. Auf eine Ammoniumberegnung tieferer Horizonte wurde verzichtet, da Ammonium unter Feldbedingungen in tieferen Bodenhorizonten nicht nachgewiesen werden konnte. Um denitrifikative $\mathrm{N}_{2}$-Emissionen des Ah- bzw. Go-GrHorizontes direkt nachweisen zu können (Kap. 4.2.7), wurden diese Horizonte bei unveränderter N-Zufuhr während der Phase II über einen Zeitraum von 47 Tagen mit ${ }^{15} \mathrm{NO}_{3}$ beregnet. Auf eine Bestimmung denitrifikativer $\mathrm{N}_{2}$-Emissionen aus dem ApHorizont mußte verzichtet werden, da keine weiteren Bodensäulen zur Verfügung standen.

\subsubsection{Transporteigenschaften der Bodensäulen für Wasser}

In den drei Bodenhorizonten stiegen die volumetrischen Wassergehalte (Vol. ${ }_{22} \mathrm{O}$ ) tendenziell von oben nach unten an (Abb. 4-4). Der untere Teil der Bodenkompartimente (jeweils $5 \mathrm{~cm}$ ) hingegen zeigte wegen der anliegenden Saugspannung bei allen Säulen wieder niedrigere Wassergehalte. Vereinfachend wurde in dieser Arbeit von homogenen Fließbedingungen in den Säulen ausgegangen. Die hydraulischen Bedingungen im Ah kamen diesen idealen 


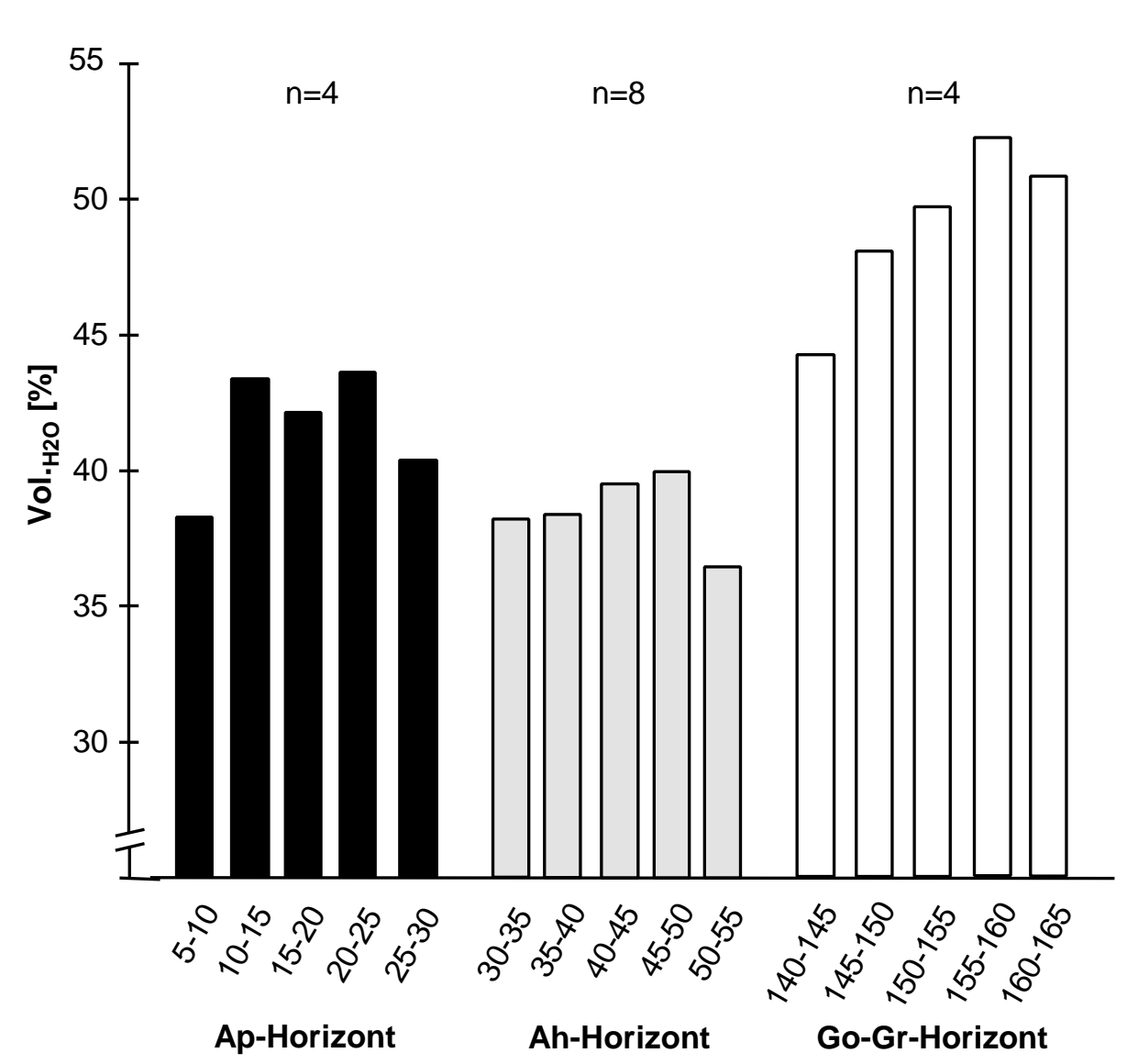

Abb.: 4-4: Volumetrische Wassergehalte (Vol. Versuchsbedingungen (Tiefenauflösung: $5 \mathrm{~cm}$ ).

Bedingungen sehr nahe; beim Go-Gr-Horizont hingegen ist der Anstieg der Vol ${ }_{\mathrm{H} 2 \mathrm{O}}$ mit zunehmender Tiefe auf eine relativ inhomogene Porengrößenverteilung zurückzuführen; hier lag jedoch ein gesättigter Wasserfluß vor.

Da bei einem Vergleich der Stickstoffdynamik unterschiedlicher Böden nicht nur die applizierten Stickstoffmengen und die Nitratfrachten, sondern auch die während der Versuchsdauer mit der Beregnungslösung ausgetauschten Porenwasservolumina (ausgetauschte PV) zu berücksichtigen sind, wurden diese in Abb. 4-5 in Abhängigkeit von der Zeit dargestellt. Unterschiedliche Porengrößenverteilungen und -strukturen verschiedener Böden führen zu einer Veränderung der Verweilzeiten von Sickerwässern, womit es zu unterschiedlichen Reaktionszeiten für chemische und mikrobiell katalysierte Prozesse kommt. Die ausgetauschten Wasservolumina der Bodensäulen (ausgetauschte PV) geben den Quotienten aus dem Porenwasservolumen (PV) und dem über die Beregnungstage kumulierten Volumen der applizierten Beregnungslösung $\left(\mathrm{BL}_{\mathrm{d}}\right)$ in den Bodensäulen der drei Horizonten wieder (Abb. 4-5, vgl. Tab. 4-3). Hierbei wird der Austausch des gesamten Bodenwassers einschließlich des Totwasseranteils unterstellt. Es wird deutlich, daß 
die in dieser Arbeit verwendeten drei Bodenhorizonte sich hinsichtlich der Transportgeschwindigkeit von Wasser nur unwesentlich voneinander unterscheiden.

Nach 55 Versuchstagen (Konditionierungsphase) waren nach einer täglichen Beregnung mit $4 \mathrm{~mm}$ einer $\mathrm{CaSO}_{4}$-Lösung $\left(0,01 \mathrm{Mol} \mathrm{L}^{-1}\right)$ (Abb. 4-5) je nach Porengrößenverteilung des jeweiligen Bodenhorizontes zwischen 1,8 und 2,2 Wasservolumina des Bodens ausgetauscht. Die selbst zu Beginn der Konditionierungsphase nahezu konstanten Perkolationsströme verdeutlichen, daß die hydraulischen Bedingungen der Bodensäulen nach Entnahme im April unter Versuchsbedingungen kaum verändert wurden.

Der fast lineare Anstieg der ausgetauschten PV macht deutlich, daß sich bei unveränderter Beregnungsmenge ein hydraulisches Gleichgewicht eingestellt hatte. In Phase I wurden bis zum 113. Versuchstag in den Bodensäulen des Ap-Horizontes 2,2 PV und in denen des Go-Gr-Horizontes 1,9 PV durch die Beregnungslösungen ausgetauscht. Nach 117 Versuchstagen waren im Ah-Horizont sogar 2,5 PV durch

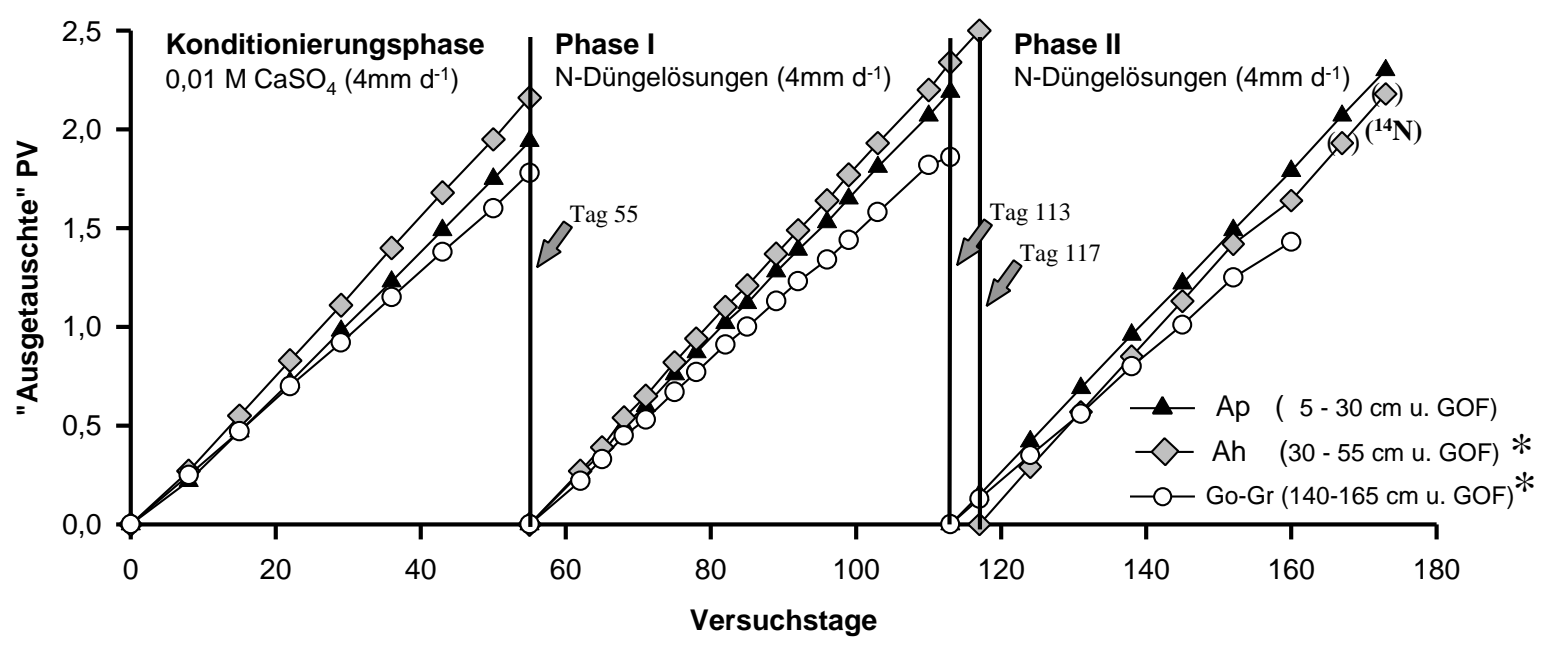

* in Phase II bis Versuchstag 160 Einsatz von ${ }^{15} \mathrm{~N}$.

Abb. 4-5: „Ausgetauschtes“ Wasservolumen der Bodensäulen (ausgetauschtes PV) unterschiedlicher Bodenhorizonte.

die Beregnung verdrängt. In Phase II wurden ähnliche Mengen an Bodenwasser ausgetauscht. Nur bei den mit ${ }^{15} \mathrm{~N}$ behandelten Bodenhorizonten (Ah $\prec-$ und Go$\mathrm{Gr}-0-)$ ) wurde die Beregnung nach Austausch von ca. 1,5 PV (Versuchstag 160) zum Zwecke von direkten Denitrifikationsmessungen abgestellt. 


\subsubsection{Nitrataustrag mit dem Perkolat}

Die Messungen zum $\mathrm{NO}_{3}$-Austrag (Abb. 4-6) erbrachten, daß zu Beginn der Konditionierungsphase für die Bodentiefen 5-30 cm (Ap - - ) und 30-55 cm u. GOF (Ah - - u. $\checkmark$ ) maximale Nitratausträge in Höhe von umgerechnet bis zu 1,7 kg ha-1 $\mathrm{d}^{-}$ 1 nachgewiesen weden konnten, während über die Perkolate der Bodensäulen aus 140-165 cm u. GOF (Go-Gr -0-) während der gesamten Konditionierungsphase kein Nitrat ausgetragen wurde. Im Verlauf der Konditionierungsphase sanken die Nitratausträge des Ap- wie auch des Ah-Horizontes. Nach Austausch von ca. 1,5 PV (Versuchstag 43) stellten sie sich auf einem Niveau von umgerechnet ca. 200 (Ap) bzw. $30 \mathrm{~g} \mathrm{ha}^{-1} \mathrm{~d}^{-1}(\mathrm{Ah})$ ein. Ammonium wurde in den Perkolaten während des gesamten Versuchszeitraumes zu keinem Zeitpunkt nachgewiesen.

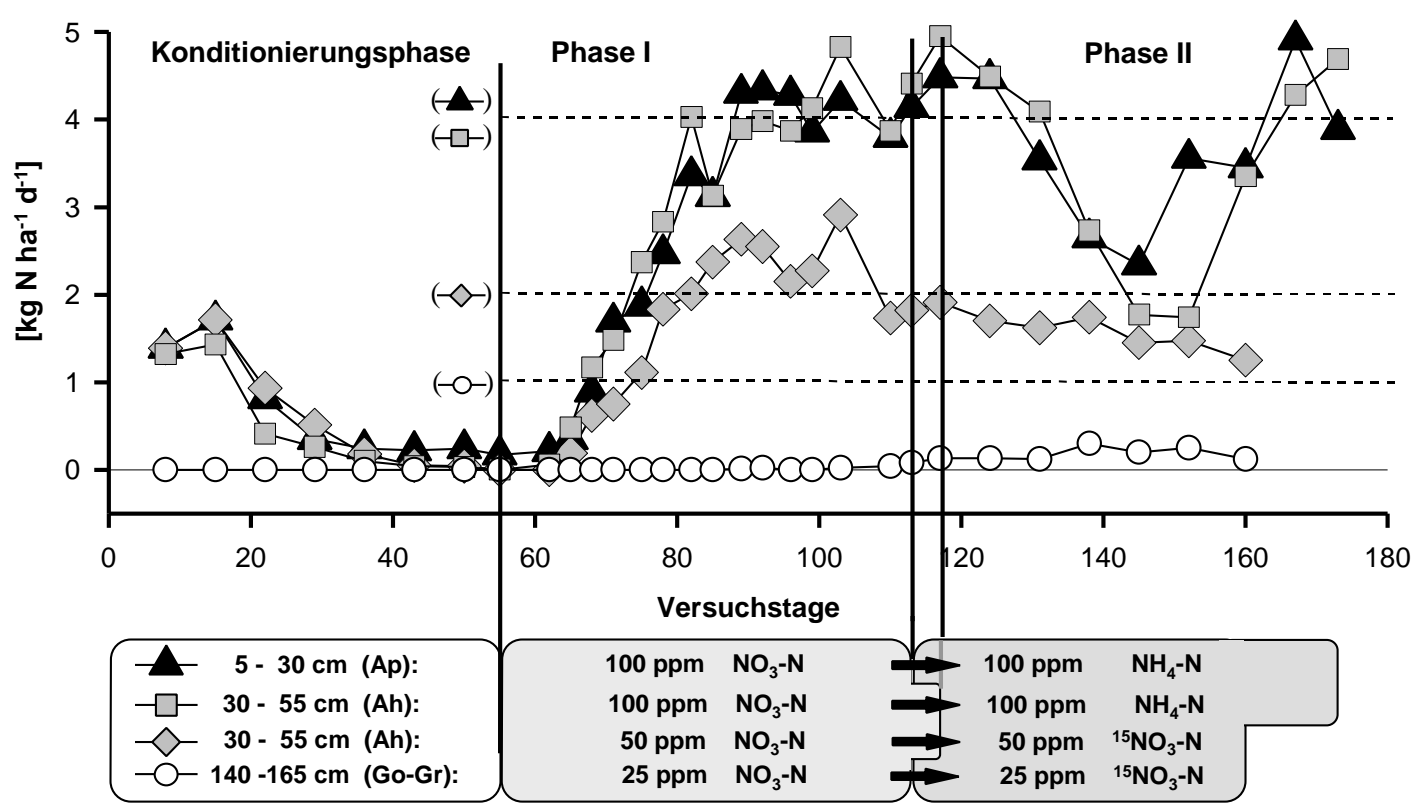

Abb. 4-6: Nitrataustragsraten mit dem Perkolat aus Bodensäulen des Ap-, Ah- und Go-GrHorizontes in Abhängigkeit der Form des applizierten N-Düngers $\left(\mathrm{NO}_{3}\right.$ und $\left.\mathrm{NH}_{4}\right)$ und der N-Inputkonzentration (----).

In der Versuchsphase I zeigten sich nach Beginn der Nitratberegnung sowohl für den Ap- $(-\boldsymbol{-})$ als auch für den Ah-Horizont ( $-\square-u$. $\diamond)$ ähnliche Durchbruchskurven, deren Steigungen und Maxima mit der Höhe der kontinuierlich applizierten Nitratmengen übereinstimmten. Für den Ap-Horizont ( $\left.\boldsymbol{\Lambda}_{-}\right)$konnte ab Austausch von ca. 1,2 PV (Tag 89) durch eine 100 ppm Nitrat-N-Lösung bei einer Beregnungsmenge von $4 \mathrm{~mm} \mathrm{~d}^{-1}\left(4 \mathrm{~kg} \mathrm{NO}_{3}-\mathrm{N} \mathrm{ha}^{-1} \mathrm{~d}^{-1}\right)$ im Mittel der Versuchstage 89 bis 113 eine mittlere Perkolatfracht von 4,14 $\mathrm{kg} \mathrm{NO}_{3}-\mathrm{N} \mathrm{ha}^{-1} \mathrm{~d}^{-1}$ nachgewiesen werden. Bei gleicher Nitratapplikation wurde für den Ah-Horizont ( $\square-$ ) bereits ab 
Austausch von ca. 1,1 PV während der Versuchstage 82-117 eine mittlere Nitratfracht über das Perkolat in Höhe von 4,11 $\mathrm{kg} \mathrm{NO}_{3}-\mathrm{N} \mathrm{ha}^{-1} \mathrm{~d}^{-1}$ festgestellt. Parallel dazu wurden im Ah-Horizont ( $-\approx$ ) bei kontinuierlicher Zugabe von umgerechnet $2 \mathrm{~kg}$ $\mathrm{NO}_{3}-\mathrm{N} \mathrm{ha}^{-1} \mathrm{~d}^{-1}$ mittlere Perkolatfrachten in Höhe von 2,24 $\mathrm{kg} \mathrm{NO}_{3}-\mathrm{N} \mathrm{ha}^{-1} \mathrm{~d}^{-1}$ ermittelt. Über das Perkolat des Ap- und Ah-Horizontes wurde also zum Ende der Phase I quantitativ sämtliches Nitrat, welches über die Beregnung zugeführt wurde, auch wieder abgeführt.

Im Gegensatz dazu konnte beim Go-Gr-Horizont (-O-) während der gesamten Phase I bei Nitratzufuhr von $1 \mathrm{~kg} \mathrm{~N}^{-1} \mathrm{~d}^{-1}$ kaum Nitrat im Perkolat nachgewiesen werden. Erst gegen Ende der Phase I deutet sich ein leichter Nitratdurchbruch an.

In Phase II wurde der Ap- und Ah-Horizont mit einer $\mathrm{NH}_{4}$-Lösung (gleiche $\mathrm{N}$ Konzentration wie in Phase I) beregnet, während in einem weiteren Versuchsansatz der Ah- und Go-Gr-Horizont mit markiertem Nitrat (gleiche Nitratkonzentration wie in Phase I) beschickt wurde.

Bei der Beregnung mit einer 50 ppm- $\mathrm{NO}_{3}-\mathrm{N}-\mathrm{Lösung}\left(2 \mathrm{~kg} \mathrm{NO}_{3}-\mathrm{N}_{\text {ha }}{ }^{-1} \mathrm{~d}^{-1}\right)$ kam es bei den Bodensäulen des Ah-Horizontes ( $\diamond)$ zu einem Rückgang der Nitratausträge. Im Mittel der Phase II konnten hier nur noch $77 \%$ der Nitratvorlage im Perkolat wiedergefunden werden (Tab. 4-5).

Tab. 4-5: N-Bilanz verschiedener Bodenhorizonte für die Versuchsphase II bei kontinuierlicher Nitratzufuhr

\begin{tabular}{|c|c|c|c|c|c|}
\hline \multirow[t]{2}{*}{$\begin{array}{l}\text { Boden- } \\
\text { horizont }\end{array}$} & \multirow{2}{*}{\multicolumn{2}{|c|}{$\begin{array}{c}\text { N-Form Applikations- } \\
\text { tage }\end{array}$}} & \multirow{2}{*}{$\begin{array}{l}\text { Zugeführte } \\
\text { N-Menge } \\
{\left[\mathrm{kg} \mathrm{ha}^{-1}\right]}\end{array}$} & \multicolumn{2}{|c|}{$\begin{array}{c}\text { Über das Perkolat } \\
\text { ausgetragene } \mathrm{N} \text {-Menge } \\
\text { relativ zum } \\
\text { zugeführten N }\end{array}$} \\
\hline & & & & {$\left[\mathrm{kg} \mathrm{ha}^{-1}\right]$} & [\%] \\
\hline Ah & $\mathrm{NO}_{3}$ & 43 & 86 & 65,78 & 76,5 \\
\hline Go-Gr & $\mathrm{NO}_{3}$ & 47 & 47 & 8,78 & 18,7 \\
\hline
\end{tabular}

Gleichzeitig ließ sich in Phase II bei fortgesetzter Nitratberegnung mit 25 ppm (1 kg $\mathrm{NO}_{3}-\mathrm{N} \mathrm{ha}^{-1} \mathrm{~d}^{-1}$ ) bei den Bodensäulen des Go-Gr-Horizontes (-o-) ein geringfügiger Nitratdurchbruch über das Perkolat erkennen, wie er sich gegen Ende der Phase I bereits ankündigte. Im Mittel dieser Go-Gr-Säulen wurden in Phase II nicht mehr als $19 \%$ des zugeführten Nitrates über die Perkolate ausgetragen (Tab. 4-5).

Nach Umstellung der Beregnung von Nitrat auf Ammonium bei gleicher Stickstoffkonzentration mit Beginn der Phase II kam es sowohl bei den Bodensäulen des Ap- $(\boldsymbol{A}-)$ als auch des Ah-Horizontes (마) zu einem deutlichen Rückgang der Nitratausträge. Nach Austausch von ca. 1,2 (Ap) bzw. 1,4 PV (Ah) (Versuchstag 145 
bzw. 152) durch die Ammoniumlösung wurden nur noch ca. $50 \%$ des applizierten Ammoniumstickstoffs, aber in Form von Nitrat-N, über das Perkolat abgeführt (Abb. 4-6). Nach Austausch von ca. 2 PV (ab Versuchstag 167) wurde quantitativ sämtliches über die Beregnung zugeführtes Ammonium- $\mathrm{N}$ im Perkolat beider Horizonte als Nitrat- $\mathrm{N}$ wiedergefunden. Wie bereits in Phase I lag auch nach Ammoniumdüngung im Perkolat dieser beiden Bodenhorizonte kein Ammoniumstickstoff vor.

Auf eine Bilanzierung der „Ammoniumphase“ wurde verzichtet, da in diesen Varianten das Bodensystem hinsichtlich der N-Flüsse zunächst nicht mehr im Gleichgewicht war.

\subsection{3 $\quad \mathrm{N}_{2} \mathrm{O}$-Flußraten}

Während des zweiten Abschnitts der Konditionierungsphase (Versuchstage 34-55) wiesen die Bodensäulen aller 3 Tiefen keine $\mathrm{N}_{2} \mathrm{O}$-Emissionen auf (Abb. 4-7).

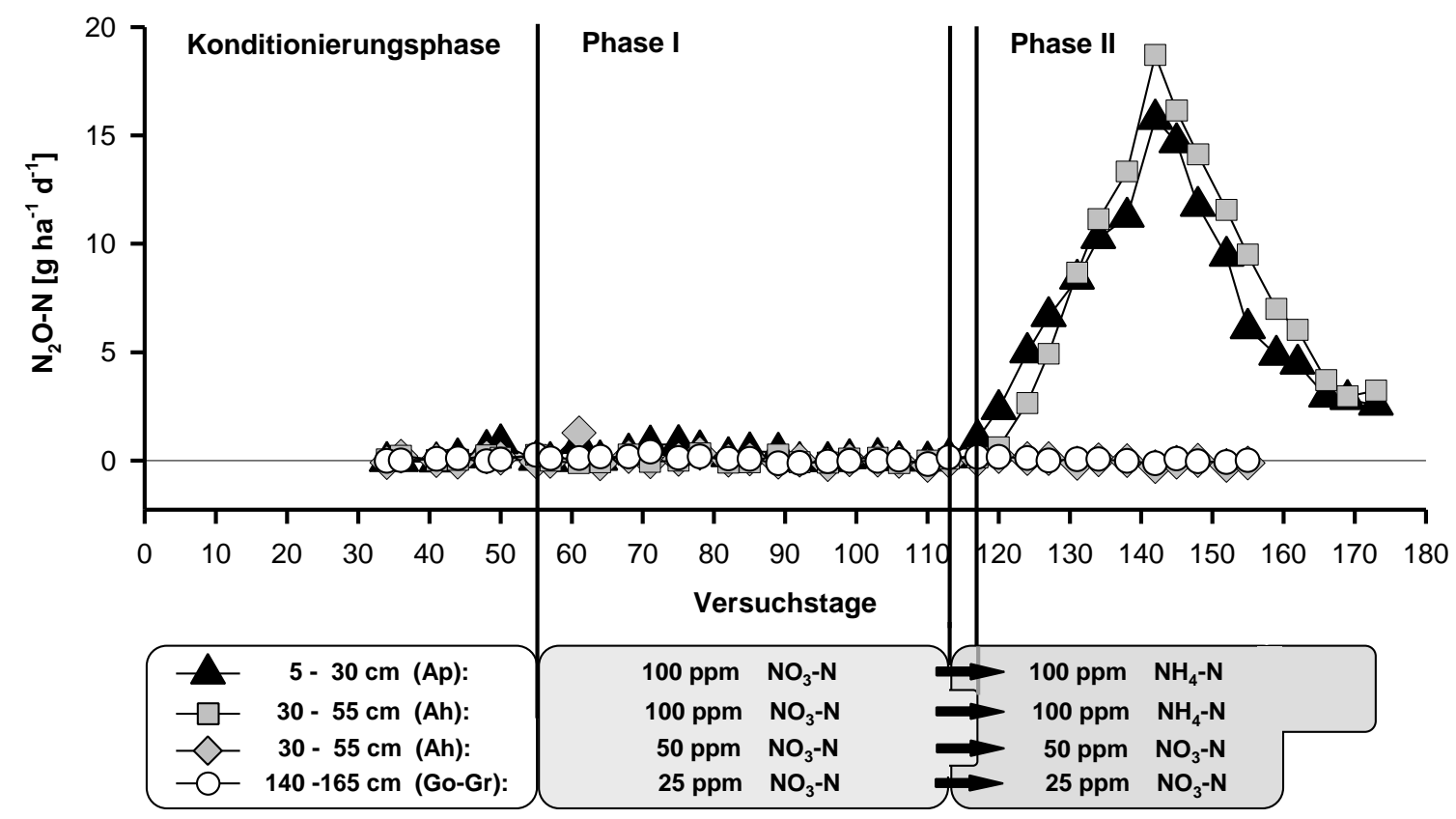

Abb. 4-7: $\quad \mathrm{N}_{2} \mathrm{O}$-Emissionsraten ungestörter Bodensäulen aus dem Ap-, Ah- und Go-Gr-Horizont in Abhängigkeit von der Form und der Menge des applizierten N-Düngers.

In der folgenden Versuchsphase I (Nitratberegnung) kam es unabhängig von der zugeführten Nitratmenge ebenfalls zu keinen $\mathrm{N}_{2} \mathrm{O}$-Emissionen. Dies setzte sich bei gleichbleibender Nitratberegnung der Horizonte 30-55 (Ah - ) und 140-165 cm u. GOF (Go-Gr -O-) auch in Phase II fort.

Erst die Umstellung auf Ammoniumberegnung führte in Phase II bei unveränderter $\mathrm{N}$-Menge (4 $\left.\mathrm{kg} \mathrm{N} \mathrm{ha}^{-1} \mathrm{~d}^{-1}\right)$ sowohl im Ap-(-) als auch im Ah-Horizont (-ㅁ) zu 
deutlichen $\mathrm{N}_{2} \mathrm{O}$-Austrägen bei einem nahezu identischen Emissionsverlauf. Bis zu einem Austausch von ca. 1 PV (Versuchstag 142) durch die Ammoniumlösung stiegen die $\mathrm{N}_{2} \mathrm{O}$-Emissionen stetig an. In beiden Bodentiefen wurden maximale Emissionen von umgerechnet 16 ( $\mathrm{Ap}-\mathbf{A}$ ) bis $19 \mathrm{~g} \mathrm{~N}_{2} \mathrm{O}-\mathrm{N}^{-1} \mathrm{~d}^{-1}$ (Ah - $-\mathrm{t}$ ) nachgewiesen. Danach gingen die Emissionen trotz stetiger $\mathrm{NH}_{4}$-Applikationen zurück und stabilisierten sich nach Austausch von ca. 2 PV in Phase II (Versuchstag 166) bei ca. $3 \mathrm{~g} \mathrm{~N}_{2} \mathrm{O}-\mathrm{N} \mathrm{ha}^{-1} \mathrm{~d}^{-1}$ (Abb. 4-7).

Bei Summierung der $\mathrm{N}$-Zufuhr und der $\mathrm{N}_{2} \mathrm{O}$-Emissionen ergibt sich, daß in Phase II dem Ap-(- - ) insgesamt $240 \mathrm{~kg} \mathrm{NH}_{4}-\mathrm{N}$ ha ${ }^{-1}$ und dem Ah-Horizont (마) $224 \mathrm{~kg}$

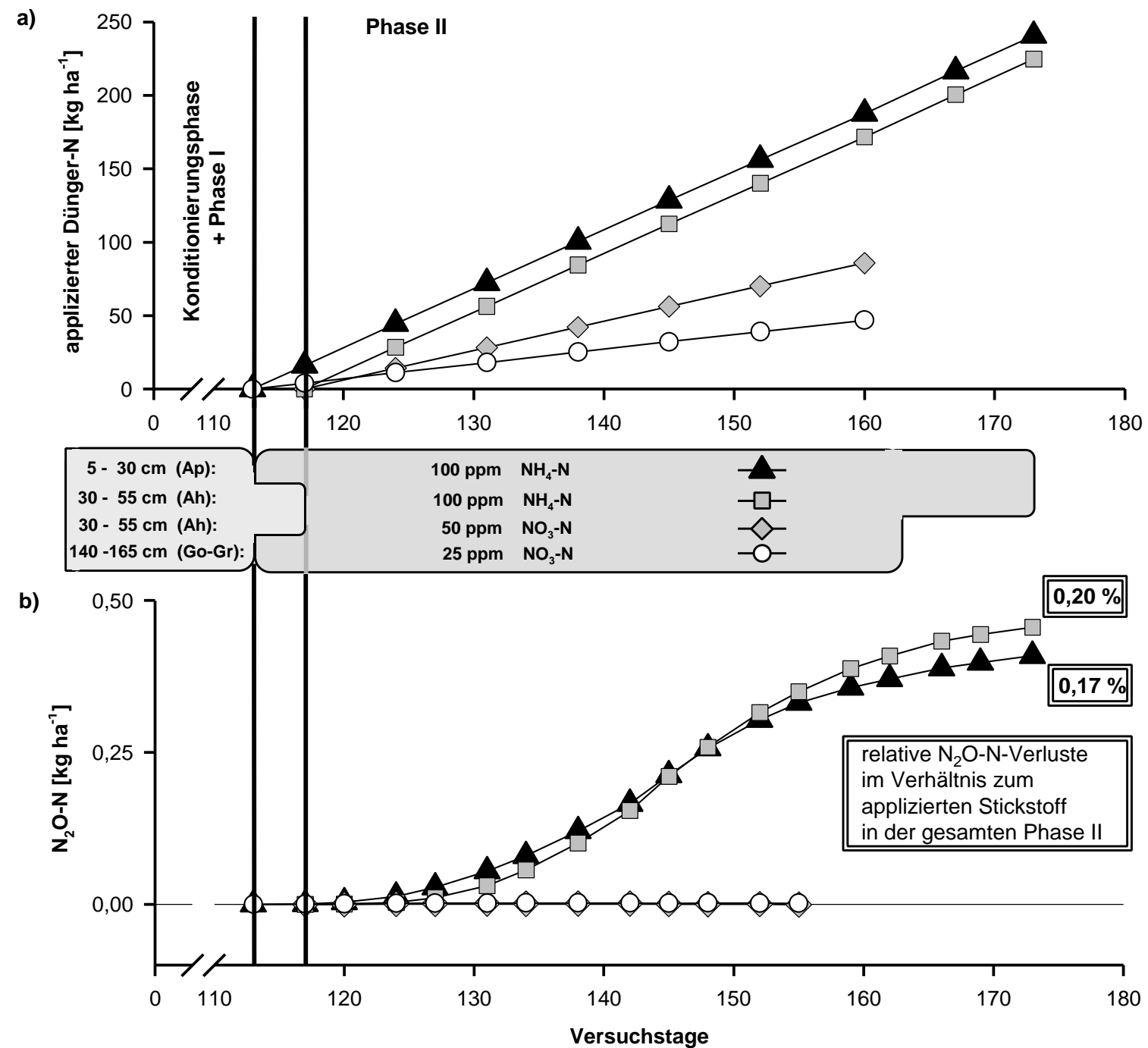

Abb. 4-8: Applizierte Stickstoffmengen (a) und $\mathrm{N}_{2} \mathrm{O}-\mathrm{N}-$ Emissionen (b) in Phase II in Abhängigkeit von der Bodentiefe und der Form des applizierten N-Düngers. 
$\mathrm{NH}_{4}-\mathrm{N}^{-1}$ zugeführt wurden (Abb. 4-8(a)). Die $\mathrm{N}_{2} \mathrm{O}-\mathrm{N}-$ Emissionen des ApHorizontes betrugen lediglich $0,17 \%$ des ausgebrachten Ammoniumstickstoffs, die des Ah-Horizontes lagen mit $0,20 \%$ in einer vergleichbaren Größenordnung (Abb. 48(b)). Die Applikation von insgesamt $86 \mathrm{~kg}$ (Ah-Horizont $\diamond$ ) bzw. $47 \mathrm{~kg} \mathrm{NO}_{3}-\mathrm{N}$ ha(Go-Gr-Horizont -O-) hingegen führte weiterhin zu keinen $\mathrm{N}_{2} \mathrm{O}$-Emissionen.

\subsection{4 $\mathrm{CO}_{2}$-Flußraten}

Zur $\mathrm{CO}_{2}$-Atmung des Bodens tragen neben vielen heterotrophen Bodenmikroorganismen auch heterotrophe Denitrifikanten bei, die zur Reduktion von Nitrat organischen Kohlenstoff veratmen. Zum Nachweis dieser mikrobiellen Aktivität wurde deshalb die $\mathrm{CO}_{2}$-Bildung der Bodensäulen quantitativ verfolgt. Es zeigte sich, daß der Ap-Horizont ( $\mathbf{A}$ ) während der Konditionierungsphase mit durchschnittlich $2 \mathrm{~kg}$ $\mathrm{CO}_{2}-\mathrm{C}$ ha $^{-1} \mathrm{~d}^{-1}$ die höchste Freisetzungsrate der drei Bodenhorizonte aufwies (Abb. 4-9). Im Ah-Horizont ( - u. $\diamond$ ) lag sie mit durchschnittlich $0,5 \mathrm{~kg} \mathrm{CO}_{2}-\mathrm{C} \mathrm{ha}^{-1} \mathrm{~d}^{-1}$ deutlich niedriger, während im Go-Gr-Horizont (-O-) keine $\mathrm{CO}_{2}$-Freisetzung stattfand.

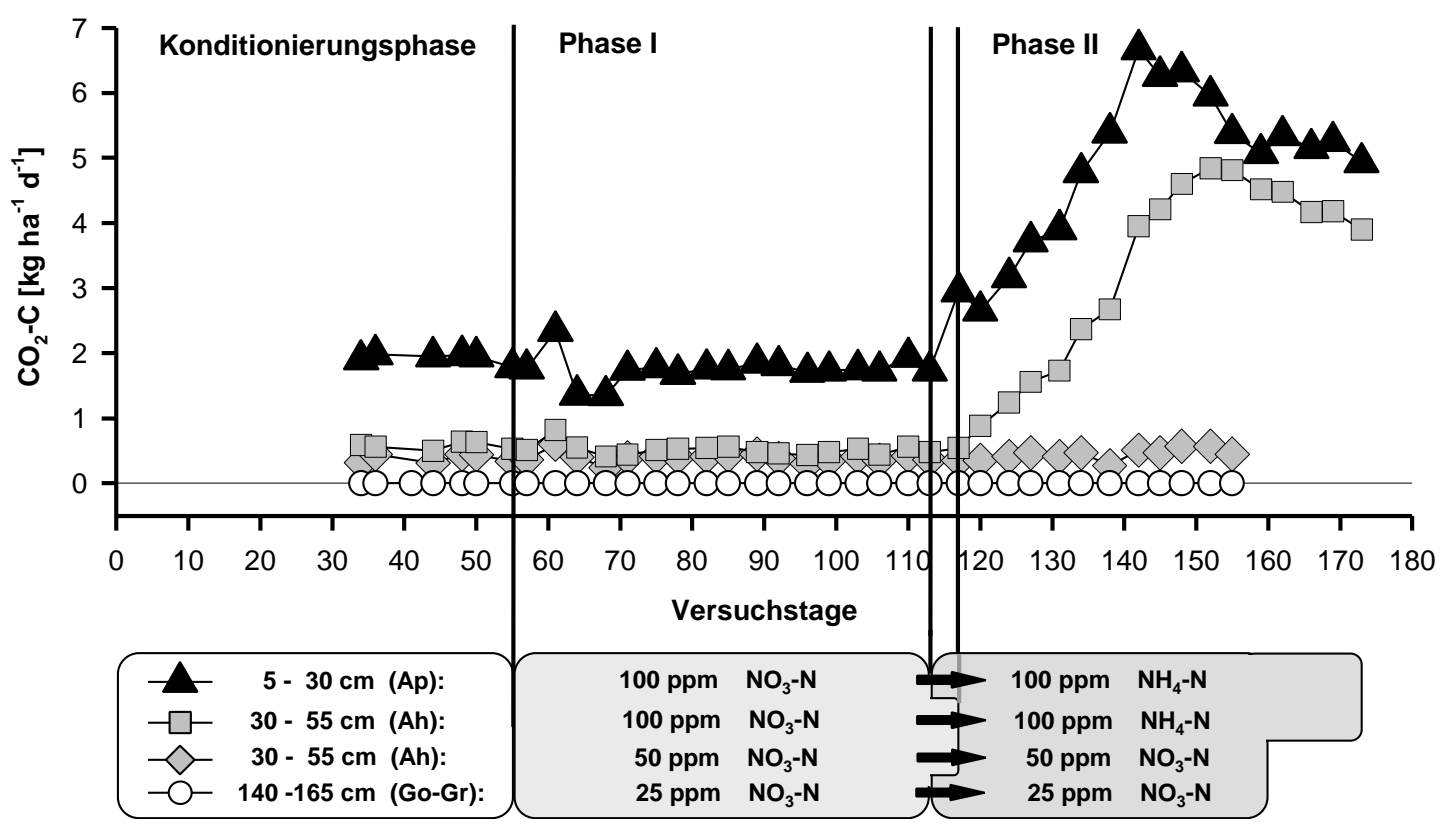

Abb. 4-9: $\quad \mathrm{CO}_{2}$-Emissionsraten ungestörter Bodensäulen des Ap-, Ah- und Go-Gr-Horizontes in Abhängigkeit von der Menge und der Form des applizierten N-Düngers.

Nach Beginn der Nitratapplikation in Phase I deutete sich aus dem Ap- $(\boldsymbol{A}-)$ und aus dem Ah-Horizont ( $-\square-$ u. $\diamond$ ) nur kurzfristig ein Anstieg der $\mathrm{CO}_{2}$-Emissionen an; im 
weiteren Verlauf blieben die Flußraten bei kontinuierlicher Nitratzufuhr nahezu unverändert. Aus dem Go-Gr-Horizont (-o-) konnte während der gesamten Versuchszeit auch bei kontinuierlicher Nitratzufuhr keine $\mathrm{CO}_{2}$-Emission nachgewiesen werden.

Die Umstellung auf Ammoniumapplikation (Phase II) führte sowohl beim Ap-( $\mathbf{A}$ ) als auch beim Ah-Horizont ( $-\square-$ zu einem deutlichen Anstieg der $\mathrm{CO}_{2}-\mathrm{C}$ Austräge bis auf Beträge von 6,7 (Ap-) bzw. 4,8 $\mathrm{kg} \mathrm{C} \mathrm{ha}^{-1} \mathrm{~d}^{-1}$ (Ah-Horizont). Nach Erreichen dieses Maximums sanken die Freisetzungsraten ab, verblieben aber, verglichen mit dem Ausgangszustand von ca. 5 (Ap-) bzw. ca. 4 kg CO${ }_{2}^{-} \mathrm{C} \mathrm{ha}^{-1} \mathrm{~d}^{-1}$ (Ah-Horizont), auf einem erhöhten Niveau. Im Mittel der Phase II zeigten sowohl der Ap- als auch der Ah-Horizont $\mathrm{CO}_{2}$-Flußraten, die um ca. $2,9 \mathrm{~kg} \mathrm{CO}_{2}-\mathrm{C}$ ha $^{-1} \mathrm{~d}^{-1}$ über den mittleren Austragsraten der Phase I lagen.

\subsection{5 $\mathrm{N}_{2}$-Emissionen}

Die Tatsache, daß über das Perkolat des Go-Gr-Horizontes (Abb. 4-6) in Phase I zunächst kein Nitrat ausgetragen wurde, legte die Vermutung nahe, daß es in diesem Horizont zu hohen gasförmigen N-Verlusten gekommen war. Um diese Vermutung zu belegen, wurden die denitrifikativen N-Emissionen des Go-GrHorizontes und zum Vergleich auch die des Ah-Horizontes direkt nachgewiesen (vgl. Kap. 4.2.7).

Während der Nitratberegnung der Bodensäulen des Ah- und Go-Gr - Horizontes kam es zu keinen $\mathrm{N}_{2} \mathrm{O}$-Emissionen (Abb. 4-7). Diese Vorkenntnisse gaben Anlaß, bei den sich an Phase II anschließenden ${ }^{15} \mathrm{~N}$ - Analysen der Inkubations - Gas-gemische auf die $\mathrm{N}_{2} \mathrm{O}$-Abtrennung (vgl. Kap. 4.2.7.2.) zu verzichten. Sporadisch durchgeführte massenspektrometrische Kontrollmessungen mit vorgeschaltetem Molsieb ließen ebenfalls kein $\mathrm{N}_{2} \mathrm{O}$ in den Gasproben dieser Bodenhorizonte erkennen. Ein Nachweis der Stickstoffmonoxid-Anteile (NO) als möglicher Teil des verbleibenden denitrifikativen ${ }^{15} \mathrm{~N}$-Anteils $\left(\mathrm{NO}+\mathrm{N}_{2}\right)$ wurden am letzten (3.) Probenahmetermin durchgeführt (vgl. Kap. 4.2.7.3). Schon die in ersten Parallelmessungen ermittelten ${ }^{15} \mathrm{~N}$-Atomprozente machten deutlich, daß es weder im Ah-Horizont noch im Go-GrHorizont zu denitrifikativen NO-Emissionen gekommen war (Vgl. Tab. 4-6). Hiermit hatte sich der weitere Einsatz eines NO-Filters als überflüssig erwiesen. 
Tab. 4-6: Indirekter Nachweis der denitrifikativen NO-Anteile beim 3. Gasprobenahmetermin

\begin{tabular}{c|c|c|c|c}
\hline Bodenhorizont & Wdhl. & \multicolumn{2}{|c|}{${ }^{15}$ N [Atom-\%] } & NO-N Atom-\% \\
& & ohne NO-Filter & mit NO-Filter & \\
\hline Ap & 1 & 0,367007 & 0,367025 & n.n. \\
Go-Gr & 1 & 0,822171 & 0,820459 & 0,00171 \\
Go-Gr & 2 & 0,438242 & 0,439396 & n.n. \\
Go-Gr & 3 & 1,035871 & 1,039146 & n.n. \\
\hline
\end{tabular}

In den ca. 8 Tagen der Inkubationsphase (Tab. 4-7) wurde im Ah-Horizont keine denitrifikative $\mathrm{N}_{2}$-Entbindung nachgewiesen. Im Headspace des Go-Gr-Horizontes hingegen ließ sich eine deutliche Anreicherung von denitrifikationsbürtigem ${ }^{15} \mathrm{~N}$ nachweisen (Abb. 4-10).

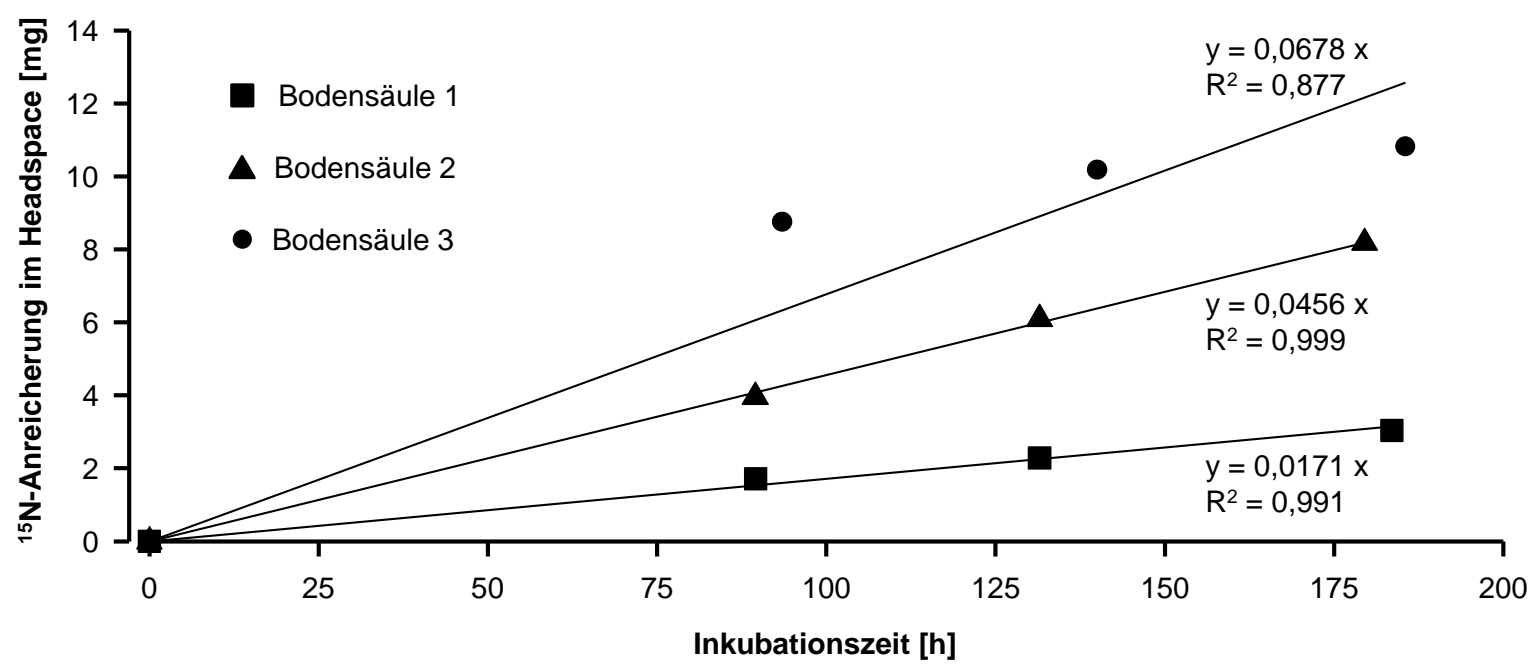

Abb. 4-10: $\quad{ }^{15} \mathrm{~N}$-Anreicherung im Headspace der Mikrokosmen des Go-Gr-Horizontes ( $\left.n=3\right)$.

Eine Linearität der ${ }^{15} \mathrm{~N}$-Anreicherung über den gesamten Inkubationszeitraum war, wie die Bodensäule 3 (Abb. 4-10) zeigt, nicht in jedem Fall gegeben. Eine Regressionsgerade 1. Ordnung spiegelt das Emissionsverhalten zum Teil nur unbefriedigend wider. Die mittlere tägliche $\mathrm{N}_{2}$-Emission während des Inkubationszeitraumes wurde deshalb aus der tatsächlichen ${ }^{15} \mathrm{~N}$-Anreicherung am Ende der Inkubation (nach ca. 8 Tagen) abgeleitet (vgl. auch Kap. 4.2.7.4). Somit 
betrug die denitrifikative $\mathrm{N}_{2}$-Emission während des Versuchszeitraumes im Go-GrHorizont im Mittel $0,59 \mathrm{~kg} \mathrm{ha}^{-1} \mathrm{~d}^{-1}$ bei einem Variationskoeffizienten zwischen den Säulen von $53 \%$ (Tab. 4-7).

Tab. 4-7: $\quad \mathrm{N}_{2}$-Emissionsraten ungestörter Bodensäulen in Abhängigkeit vom Bodenhorizont und der zuvor applizierten Nitratmenge

\begin{tabular}{c|c|c|c|c}
\hline Bodenhorizont & $\mathbf{n}$ & $\begin{array}{c}\text { zuvor } \\
\text { appliziertes } \\
{ }^{15} \mathrm{NO}_{3}-\mathbf{N} \\
{\left[\mathbf{k g ~ h a}^{-1} \mathbf{d}^{-1}\right]}\end{array}$ & $\begin{array}{c}\mathbf{N}_{2^{-}} \\
\text {Emissionen }\end{array}$ & VK \\
{$\left[\mathbf{k g ~ h a}^{-1} \mathbf{d}^{-1}\right]$} & {$[\%]$} \\
\hline Ah & 4 & 2 & $\mathrm{n} . \mathrm{n}$. & - \\
Go-Gr & 3 & 1 & 0,59 & 53,3 \\
\hline
\end{tabular}

Zusammenfassend kann festgestellt werden, daß es unter Versuchsbedingungen nur im Go-Gr-Horizont zu starken denitrifikativen $\mathrm{N}_{2}$-Abgaben kam, während gleichzeitig keine denitrifikativen NO- und $\mathrm{N}_{2} \mathrm{O}$-Emissionen nachzuweisen waren. 


\subsection{Diskussion}

Untersuchungen über die Bedeutung des WRaps-Anbaus für die $\mathrm{N}_{2} \mathrm{O}$ - und $\mathrm{CH}_{4}{ }^{-}$ Flüsse eines Gley-Auenbodens führten u. a. zu dem Ergebnis, daß auf dem Standort im Vergleich zu Erkenntnissen aus Versuchen mit ähnlichen Anbausystemen auf anderen Bodentypen (FLESSA et al., 1998) wesentlich geringere $\mathrm{N}_{2} \mathrm{O}$-Emissionen auftraten. LICKFETT (1993) fand auf demselben Gley-Auenboden der vorliegenden Untersuchungsergebnisse insbesondere in Bodentiefen $>120 \mathrm{~cm}$ unter GOF wesentliche N-Defizite, die er N-Verlusten durch Denitrifikation zuordnete. Dieser Kenntnisstand war Anlaß für eingehende Modelluntersuchungen zur Erforschung der $\mathrm{N}$-Transformationsprozesse im Ap-, Ah- und Go-Gr-Horizont des Gley-Auenbodens unter kontrollierten Bedingungen in der Mikrokosmenanlage (Kap. 4.2.2). In den Untersuchungen wurden sowohl die gasförmigen $\mathrm{N}_{2} \mathrm{O}$-Emissionen unterschiedlicher Bodenhorizonte in Abhängigkeit von der applizierten N-Form als auch die denitrifikativen $\mathrm{N}_{2}$-Emissionen des Ap- und Go-Gr-Horizontes direkt bestimmt.

\section{Nitrataustrag aus den Bodensäulen}

Die nahezu konstanten Perkolationsflüsse aller Bodensäulen wiesen bereits zu Beginn der Konditionierungsphase auf einen stationären Fluß hin (Abb. 4-5). Die Porenwasservolumina in den Säulen (PV) waren zwar bei gleicher Beregnungsmenge in Abhängigkeit von der Porengrößenverteilung unterschiedlich hoch, unterschieden sich jedoch nicht wesentlich voneinander. Rechnerisch ergab sich deshalb mit ca. 24 (Ah-), 26 (Ap-) sowie 30 Tagen (Go-Gr-Horizont) ein unterschiedlicher Zeitbedarf für den Austausch eines Porenwasservolumens (PV) (Tab. 4-3).

Nach Austausch von ca. 1,5 PV durch die $\mathrm{CaSO}_{4}$-Lösung stellte sich in der Konditionierungsphase nach 43 Versuchstagen ein konstanter mineralischer $\mathrm{N}$ Austrag in Höhe von umgerechnet $200 \mathrm{~g} \mathrm{ha}^{-1} \mathrm{~d}^{-1}$ im Ap-Horizont und von ca. $30 \mathrm{~g}$ $h^{-1} d^{-1}$ im Ah-Horizont ein. In den Perkolaten wurde während der Konditionierungsphase ausschließlich Nitrat nachgewiesen. Dies weist auf ein hohes Mineralisations- und Nitrifikationsvermögen der Bodensäulen hin. Die nahezu konstanten täglichen N-Frachten lassen vermuten, daß sich die Bodensäulen hinsichtlich ihrer N-Dynamik zu diesem Zeitpunkt in einem "steady state“ befanden. Unter der Annahme, daß der Gesamt-N-Anteil $\left(\mathrm{N}_{\mathrm{t}}\right)$ des Bodens sich nicht veränderte, entspricht die konstante $\mathrm{NO}_{3}$-Fracht annäherungsweise der N-Nettomineralisations- 
rate $^{1)}$ der betreffenden Bodenhorizonte, da den Systemen von außen kein Stickstoff zugeführt wurde.

Nach Austausch von ca. 1,8 PV während der gesamten Konditionierungsphase lag bei den Bodensäulen des Go-Gr-Horizontes offensichtlich keine Nettomineralisation vor, da keine Nitratausträge festgestellt werden konnten (vgl. Abb. 4-6). Trotz fehlender Nettomineralisation können jedoch gasförmige N-Verbindungen freigesetzt werden, wenn Bruttomineralisation erfolgt. Da aber trotz eines hohen $\mathrm{C}_{\text {org }}$ - Gehaltes von $1,42 \%$ sowie eines engen $\mathrm{C} / \mathrm{N}$-Verhältnisses von ca. 13 (Tab. 3-1) während der gesamten Versuchsdauer kein $\mathrm{CO}_{2}$ emittiert wurde (vgl. Abb. 4-9), ist eine vermehrte Aktivität heterotropher Mikroorganismen und somit eine Stickstofffreisetzung auf diesem Wege auszuschließen. Neben dem anzunehmenden niedrigen Sauerstoffpartialdruck bei $93 \%$ WFPS kommt für heterotrophe Mikroorganismen ferner maßgebend hinzu, daß die hohen $\mathrm{C}_{\text {org }}$ - Gehalte dieses Tiefenabschnittes auf einen fossilen Ah-Horizont zurückzuführen sind, in dem keine mikrobiell leicht umsetzbare organische Substanz zu erwarten ist.

Nach Beginn der Nitratzufuhr (Phase I) zeigten sich sowohl für den Ap- als auch für den Ah-Horizont Durchbruchskurven des Nitrats (Abb. 4-6), wie sie von BEESE und VAN der PLOEG (1979) auch für andere Böden beschrieben wurden. Da dem System kontinuierlich Nitrat zugeführt wurde, bildeten die Nitratfrachten ein hohes Plateau. In den Perkolaten des Ap- wie des Ah-Horizontes wurde in Phase I nach Austausch von 1,2 (Ap) bzw. 1,1 PV (Ah) mehr Nitrat gefunden, als zugeführt wurde (Abb. 4-5 und 4-6). Anhand von Input-Output-Bilanzen wird häufig versucht, die Denitrifikationsraten in Böden zu quantifizieren (PRATT et al., 1972; Voss, 1985; SCHUlte-Kellinghaus, 1987; MAidl und FischbeCK, 1987; HeYder, 1993; FunK, 1993). Hierbei muß aber die natürliche Mineralisationsrate ${ }^{2)}$ des Bodens berücksichtigt werden. Sie betrug im Ap 0,20 und im Ah 0,03 $\mathrm{kg} \mathrm{NO}_{3}-\mathrm{N} \mathrm{ha}^{-1} \mathrm{~d}^{-1}$ (s.o.). Außerdem muß eine mögliche Steigerung der $\mathrm{N}$-Mineralisation aufgrund eines Priming-Effektes nach Zugabe von Dünger- $\mathrm{N}$ in Betracht gezogen werden. Er wird mit einer Stimulierung der mikrobiellen Aktivität infolge einer N-Düngung begründet (WeStermanN und KURTZ, 1973; WestermanN und TUCKER, 1974; WoOds et al., 1987). Da sich jedoch nach Nitratzugabe zu Beginn der Phase I sowohl im Ap-als auch im Ah-Horizont lediglich kurzfristig eine leichte Erhöhung der $\mathrm{CO}_{2}$ Freisetzungsrate zeigte (Abb. 4-9), wird angenommen, daß es nur zu einer geringen und kurzfristigen Steigerung der Mineralisationsrate kam. Do NGOC (1975)

1) Unter der N-Nettomineralisation wird die Menge pflanzenverfügbaren Stickstoffs $\left(\mathrm{NO}_{3}\right.$ und $\left.\mathrm{NH}_{4}\right)$ verstanden, die nahezu ausschließlich aus den im Boden vorhandenen organischen $\mathrm{N}$ Verbindungen über den mikrobiellen Bedarf des Bau- und Betriebsstoffwechsels hinaus mineralisiert wird (BARTHOLOMEW UND CLARK, 1950; JANSSON, 1958; ZÖTTL, 1960).

2) hier abgeleitet aus dem Nitrat-N-Austrag gegen Ende der Konditionierungsphase 
errechnete nach einer N-Düngung von 180 und $350 \mathrm{~kg} \mathrm{~N}$ ha $^{-1}$ eine Steigerung der Mineralisationsrate um das 1,6- bzw. 3fache gegenüber ungedüngtem Boden. Den Bodensäulen der Mikrokosmenanlage wurden in Phase I $115 \mathrm{~kg}\left(50 \mathrm{ppm} \mathrm{NO}{ }_{3}-\mathrm{N}\right)$ und $230 \mathrm{~kg}$ Stickstoff ( $100 \mathrm{ppm} \mathrm{NO}-\mathrm{N}$ ) pro Hektar zugeführt. Bei Erwartung einer Verdoppelung der Mineralisationsrate zur Zeit der Nitratzugabe müßten in Phase I im Ap-Horizont maximale $\mathrm{N}$-Mineralisationsraten von etwa $0,4 \mathrm{~kg} \mathrm{~N}^{-1} \mathrm{~d}^{-1}$ sowie im Ah-Horizont von etwa $0,06 \mathrm{~kg} \mathrm{~N}^{-1} \mathrm{~d}^{-1}$ auftreten. Nach Abzug dieser maximal anzunehmenden N-Mineralisationsraten von den täglichen Nitratfrachten errechnet sich nach Austausch von $>1$ PV durch die Beregnungslösung ein Nitrat-Input, wie er im Experiment eingesetzt wurde (4 bzw. $2 \mathrm{~kg} \mathrm{NO}_{3}-\mathrm{N} \mathrm{ha}^{-1} \mathrm{~d}^{-1}$ ) (vgl. Abb. 4-6). Im Apwie auch im Ah-Horizont sind demnach keine größeren denitrifikativen Stickstoffverluste zu erwarten. Unter Versuchsbedingungen ist daher die Denitrifikationsleistung dieser Bodensäulen zu vernachlässigen.

Demgegenüber wurden im Perkolat des Go-Gr-Horizontes nach Austausch von 1,9 PV (Versuchstag 113, Phase I) durch eine 25 ppm Nitrat-N-Lösung (kontinuierliche Zugabe von $1 \mathrm{~kg} \mathrm{NO}_{3}-\mathrm{N} \mathrm{ha}^{-1} \mathrm{~d}^{-1}$ ) Nitrat nur in Spuren nachgewiesen (Abb. 4-6). Dieses Ergebnis läßt in diesem Horizont hohe gasförmige N-Verluste erwarten. Der mittlere WFPS von $93 \%$ ist ein weiteres Indiz für Denitrifikationsverluste.

Bei der über Phase I hinaus fortgesetzten Nitratdüngung (50 ppm $\mathrm{NO}_{3}-\mathrm{N}-\mathrm{Lösung}$ ) des Ah-Horizontes zeigte sich in Phase II ein Rückgang des Nitrataustrages. Im Mittel konnten nur $77 \%$ (Tab. 4-5, Phase II) der Nitratvorlage im Perkolat wiedergefunden werden. Anhand der Untersuchungen konnte nicht nachgewiesen werden, ob hierfür eine mikrobielle $\mathrm{NO}_{3}{ }^{-}$-Immobilisation verantwortlich ist. Sowohl ARDAKANI et al. (1975) als auch HyNES und KNOWLES (1980) berichten, daß in Langzeitversuchen, in denen dem System kontinuierlich Nitrat zugeführt wird, eine mikrobielle $\mathrm{NO}_{3}$-Immobilisation ohne Bedeutung ist. Die Autoren weisen aber darauf hin, daß allerdings in $\mathrm{N}$-limitierten Systemen $\mathrm{NO}_{3}$ mikrobiell immobilisiert werden kann. Unter diesem Aspekt wird für die vorliegenden Versuche eine N-Immobilisation des Ah-Horizontes in Phase II nicht ausgeschlossen. Anhand der durchgeführten NBilanzierung in Phase II wäre deshalb beim Ah-Horizont von einer biologischen Immobilisation und gasförmigen N-Verlusten in Höhe von $23 \%$ des zugegebenen Stickstoffs auszugehen. Eventuelle gasförmige Stickstoffverluste dieses Horizontes sollen jedoch im weiteren Verlauf der Arbeit direkt nachgewiesen werden.

Im Go-Gr-Horizont in Phase II konnte über die N-Bilanz der Verbleib von durchschnittlich $81 \%$ des applizierten Nitrats nicht geklärt werden (vgl. Tab. 4-5). In diesem Bodenhorizont muß von erheblichen gasförmigen $\mathrm{N}$-Verlusten ausgegangen 
werden, wie es im späteren Teil der Arbeit noch direkt nachgewiesen wird. Eine organotrophe Denitrifikation (vgl. Kap. 2.1.2) kann jedoch nicht als einzige Ursache für diese massiven $\mathrm{N}$-Verluste herangezogen werden, da dies auch zu einem Anstieg der Bodenatmung im Go-Gr-Horizont hätte führen müssen, was jedoch nicht der Fall war (Abb. 4-9).

Während der Ammoniumdüngung des Ap und Ah wird die Leistungsfähigkeit der Nitrifikanten in diesen Horizonten dadurch verdeutlicht, daß nach Austausch von ca. 2 PV mit einer Ammoniumlösung (Versuchstag 167, Phase II) kein Ammonium im Perkolat gefunden wurde. Ca. 45 Tage nach Beginn der $\mathrm{NH}_{4}$-Zugabe wurden sogar mehr als $100 \%$ des kontinuierlich applizierten Ammoniumstickstoffs als Nitrat im Perkolat wiedergefunden (Abb. 4-6). Dieses kann damit erklärt werden, daß nach der vollständigen Adaptation der Nitrifikanten an die neuen Milieubedingungen zusätzlich zu dem kontinuierlich zugeführten Ammonium auch ein Teil des zuvor an den Austauschern gebundenen Ammoniums nitrifiziert wurde.

Ein zusätzlicher Hinweis auf eine hohe Nitrifikationsleistung dieser Bodensäulen ist die Tatsache, daß es in Phase II bei Ammoniumzugabe im Gegensatz zur Nitratdüngung in Phase I zu einem deutlichen Anstieg der $\mathrm{CO}_{2}$-Emissionen kam (Abb. 4-9). Da sowohl der Ap- als auch der Ah-Horizont $\mathrm{CaCO}_{3}$-Gehalte in Höhe von ca. $5 \%$ aufwiesen (Tab. 3-1), dürften diese $\mathrm{CO}_{2}$-Freisetzungsraten eine Folge der durch den nitrifikativen Protoneneintrag beschleunigten Auflösung freien Carbonates sein. Bei einer täglichen Zufuhr von $4 \mathrm{~kg} \mathrm{NH}_{4}-\mathrm{N} \mathrm{ha}^{-1}$ ist ein Anstieg der carbonatbürtigen $\mathrm{CO}_{2}-\mathrm{C}$-Emissionen des Bodens um bis zu 3,5 $\mathrm{kg} \mathrm{ha}^{-1} \mathrm{~d}^{-1} \mathrm{zu}$ erwarten. Die im Mittel um 2,9 kg ha-1 $\mathrm{d}^{-1}$ höheren $\mathrm{CO}_{2}$-C-Austräge nach Ammoniumdüngung in Phase II (Kap. 4.3.4) sind damit vermutlich in voller Höhe auf den nitrifikativen Protoneneintrag zurückzuführen.

Der deutliche Rückgang des Nitrataustrages nach Umstellung der Nitratdüngung auf Ammoniumdüngung ist auf die Auswaschung des in Phase I zugegebenen Nitrats zurückzuführen (vgl. Abb. 4-6). Der folgende Wiederanstieg ist mit dem verzögerten Durchbruch des zugeführten Ammoniumstickstoffs zu erklären. MOCHOGE und BEESE (1983) stellten bei Perkolationsversuchen einer Parabraunerde fest, daß der $\mathrm{NO}_{3}$ Durchbruch bei $\mathrm{NH}_{4}$-Düngung im Vergleich zu einer $\mathrm{NO}_{3}$-Düngung sich um etwa 10 Tage verzögerte. Es wurde vermutet, daß dieses durch die Adsorption von $\mathrm{NH}_{4}{ }^{+}$ an den Austauschern sowie eine eventuell verzögerte Nitrifikation bedingt war. In diesem Versuch kam es, verglichen mit der $\mathrm{NO}_{3}$-Applikation in Phase I, zu einer Verzögerung des Nitratdurchbruchs um ca. 16 Tage, was ebenfalls auf eine hohe Nitrifikationsleistung schließen läßt. Der Rückgang der $\mathrm{NO}_{3}$-Austräge beim Wechsel von $\mathrm{NO}_{3}-\mathrm{zu} \mathrm{NH} \mathrm{NH}_{4}$-Beregnung im Ap- und im Ah-Horizont ist somit auch Ausdruck einer verzögerten Nitrifikation und kann mit einer Aufsättigung der Austauscher mit 
$\mathrm{NH}_{4}$ oder einer Anpassung der Nitrifikanten an die hohe, kontinuierliche $\mathrm{NH}_{4}{ }^{-}$ Applikation erklärt werden.

Die Tatsache, daß ca. 46 Tage nach Beginn der Ammoniumzugabe, also nach Austausch von ca. 2 PV durch eine 100 ppm NH$_{4}$-Lösung, quantitativ sämtlicher Stickstoff in den Perkolaten wiedergefunden wurde, macht deutlich, daß es nach Ammoniumzufuhr weder im Ap- noch im Ah-Horizont zu nennenswerten gasförmigen $\mathrm{N}-$ Verlusten gekommen sein kann.

\section{$\mathrm{N}_{2} \mathrm{O}$-Flüsse}

Aus den ungestörten Bodensäulen wurde bei mittleren wassergefüllten Porenvolumina von ca. $82 \%$ (Ap-) bzw. $77 \%$ (Ah-Horizont) (Tab. 4-2) während der Konditionierungsphase kein $\mathrm{N}_{2} \mathrm{O}$ emittiert (Abb. 4-7).

GRANLI und BOCKMANN (1994) stellen den WFPS als einen maßgeblichen Parameter dar, um die Bedingungen einer maximalen Produktion und Emission von $\mathrm{N}_{2} \mathrm{O}$ unabhängig von der Bodenart zu beschreiben. Nach Kenntnis der Autoren werde häufig die Feldkapazität als das Maß der Bodenfeuchte angegeben, bei der die höchsten $\mathrm{N}_{2} \mathrm{O}$-Emissionen auftreten. Im Gegensatz zu den eigenen Untersuchungen berichten KLEMEDTSSON et al. (1988) und HANSEN et al. (1993) von hohen $\mathrm{N}_{2} \mathrm{O}$ Emissionen bei einem WFPS > $75 \%$. PARTON et al. (1988), KLEMEDTSSON et al. (1988), SCHUSTER und KONRAD (1992) und DAVIDSON (1991, 1992) berichten von maximalen $\mathrm{N}_{2} \mathrm{O}$-Emissionen bei einem WFPS im Bereich von 45 bis $75 \%$, da in diesem Bereich sowohl die Nitrifikanten als auch die Denitrifikanten $\mathrm{N}_{2} \mathrm{O}$ Produzenten sein können. DAVIDSON (1991) weist jedoch ausdrücklich darauf hin, daß sich das Maximum der $\mathrm{N}_{2} \mathrm{O}$-Emissionen in Abhängigkeit von Bodentyp und Bodeneigenschaften verschieben kann.

$\mathrm{Da}$ in den Bodensäulen des Ap- und Ah-Horizontes während der Konditionierungsphase ständig Stickstoff nachgeliefert wurde (s.o.), ist nicht davon auszugehen, daß die als Hauptverursacher der $\mathrm{N}_{2} \mathrm{O}$-Bildung im Boden geltenden Stoffwechselprozesse der Nitrifikation und der Denitrifikation (vgl. Kap. 2.1.2) aufgrund von Stickstoffmangel zum Erliegen kamen. Gründe dafür, daß die Bodensäulen keine $\mathrm{N}_{2} \mathrm{O}$-Basisemissionen zeigten, können daher nur sein, daß entweder bei den beiden Stoffwechselvorgängen $\mathrm{N}_{2} \mathrm{O}$ gar nicht erst als Endprodukt im Boden auftritt oder daß einmal produziertes $\mathrm{N}_{2} \mathrm{O}$ auf dem Weg zur Bodenoberfläche in Abhängigkeit von den partiellen bodenphysikalischen und bodenchemischen Gegebenheiten weiter zum molekularen Stickstoff reduziert wird.

Bei Nitratapplikation wurde in Phase I und Phase II aus dem Ap- und Ah-Horizont kein $\mathrm{N}_{2} \mathrm{O}$ emittiert (Abb. 4-7), obwohl nach DAVIDSON (1991) der Wassergehalt des 
Bodens für die Möglichkeit denitrifikativer $\mathrm{N}_{2} \mathrm{O}$-Emissionen spricht. Diese Feststellung steht im Gegensatz zu den Mitteilungen von MosIER et al. (1983), LIND (1985), EATON und PARIQUIN (1989) sowie MATSON et al. (1990), nach denen steigende Nitratgehalte des Bodens mit erhöhten $\mathrm{N}_{2} \mathrm{O}$-Emissionen einhergehen. MOSIER et al. (1983) stellten sogar einen prinzipiellen Zusammenhang zwischen dem Bodennitratgehalt und gasförmigen N-Verlusten des Bodens her; bei zunehmendem Bodennitratgehalt nähmen die denitrifikationsbürtigen $\mathrm{N}_{2} \mathrm{O}$-Emissionen fast linear zu. Grund für die gegensätzlichen Ergebnisse der eigenen Untersuchungen könnte u.a. der konstante Wassergehalt in den Bodensäulen während der Versuchsdurchführung sein, denn MULVANEY und KURTZ (1984) beobachteten höchste Denitrifikationsraten nur bei einem Wechsel zwischen Austrocknen und Wiederbefeuchten des Bodens. Die Autoren weisen darauf hin, daß die Denitrifikationsraten zurückgehen, wenn der Boden über einen längeren Zeitraum einen höheren Wassergehalt aufweist.

So ist zu vermuten, daß sowohl die Bodensäulen des Ap- als auch des AhHorizontes nahe Feldkapazität noch genügend kontinuierlich luftführende Poren für einen guten Gasaustausch aufwiesen, so daß ein relativ hoher Sauerstoffpartialdruck in den gesamten Bodensäulen gewährleistet war. Dieses hätte geringe Denitrifikationsraten zur Folge mit entsprechend geringen Emissionen an denitrifikationsbürtigem $\mathrm{N}_{2} \mathrm{O}$.

Im Go-Gr-Horizont wiesen die beträchtlichen $\mathrm{NO}_{3}$-Verluste auf Denitrifikation hin (s.o.). Dennoch wurden auch für diesen Bodenhorizont keine $\mathrm{N}_{2} \mathrm{O}$-Emissionen nachgewiesen (Abb. 4-7). Dieses könnte im Verhältnis von emittiertem $\mathrm{N}_{2} \mathrm{O}$ zu emittiertem $\mathrm{N}_{2}$ bedingt sein. Das $\mathrm{N}_{2} \mathrm{O} / \mathrm{N}_{2}$-Verhältnis nimmt nach ERIKSEN und HOLTEM-HARTWIG (1993) und BURESH et al. (1993) mit steigendem Wassergehalt des Bodens ab; es zeigen sich höchste Werte, wenn die Denitrifikationsrate in Verbindung mit niedrigen Wassergehalten des Bodens am geringsten ist (ROLSTON et al., 1982; AulaKH et al., 1984; Christensen, 1985; MuraKamı und KumAZAWA, 1987; SCHUSter und ConRAD, 1992; Weier et al., 1993a). Weiterhin ist eine Zunahme der Nitratatmung unter stark anaeroben Bedingungen in Betracht zu ziehen, da unter Nitratmangel nach Mosier et al. (1993) eine Ausnutzung des Reduktionspotentials bis zum $\mathrm{N}_{2}$ erfolgt. Durch Wassergehalte oberhalb der Feldkapazität, die zu physikalischen Diffusionsbarrieren führen und das Entweichen von $\mathrm{N}_{2} \mathrm{O}$ aus dem Boden verhindern, werde dieser Prozeß gefördert (CHRISTENSEN, 1985; SHEPHERD et al., 1991). Je länger $\mathrm{N}_{2} \mathrm{O}$ im Boden verweile, desto mehr werde davon weiter zum molekularen Stickstoff $\left(\mathrm{N}_{2}\right)$ reduziert.

Nach Umstellung der N-Zufuhr auf Ammoniumberegnung (Phase II) wurden aus dem Ap- und Ah-Horizont sofort deutliche Mengen an $\mathrm{N}_{2} \mathrm{O}$ freigesetzt (Abb. 4-7). Da 
es bei ausschließlicher Nitratdüngung dieser Säulen keine (denitrifikativen) $\mathrm{N}_{2} \mathrm{O}$ Emissionen gab, können die Emissionen nach Ammoniumdüngung nur nitrifikationsbürtig sein. Auch BLACKMER und BREMNER (1981) sowie CONRAD et al. (1983) schreiben die Entstehung von $\mathrm{N}_{2} \mathrm{O}$ nach $\mathrm{NH}_{4}{ }^{+}$-Düngung hauptsächlich dem oxidativen Stoffwechselweg zu. $\mathrm{N}_{2} \mathrm{O}$ kann nach PAPEN und RENNENBERG (1990) bei der Ammoniumoxidation sowohl aus den Zwischenprodukten Hydroxylamin $\left(\mathrm{NH}_{2} \mathrm{OH}\right)$ und Nitroxyl (HNO) entstehen und bei partiellem Sauerstoffmangel durch eine sogenannte Nitrifizierer-Denitrifikation (GROFFMAN, 1991) direkt aus $\mathrm{NO}_{2}{ }^{-}$gebildet werden. Auch RACKWITZ (1996) beobachtete in einem Mikrokosmenversuch bei ähnlichen Bodenwassergehalten wie in den vorliegenden Versuchen, daß es nach einer einmaligen $\mathrm{NH}_{4}{ }^{+}$-Düngung in allen untersuchten Böden zu einem sofortigen Anstieg der $\mathrm{N}_{2} \mathrm{O}$-Emissionen kam. Diese wurden ebenfalls auf die Nitrifikation zurückgeführt. Der Autor berichtet, daß in Abhängigkeit von der Bodenart nach Austausch von 0,25 bis 1 PV durch Beregnungswasser infolge der Ammoniumdüngung ein Maximum erreicht wurde, danach fielen die Emissionen bei zurückgehender $\mathrm{NH}_{4}{ }^{+}$-Konzentration in der Bodenlösung kontinuierlich bis auf Null ab. Die parallel dazu gestiegenen Nitratkonzentrationen in der Bodenlösung hatten hingegen keinen Anstieg denitrifikativer $\mathrm{N}_{2} \mathrm{O}$-Emissionen zur Folge.

Die $\mathrm{N}_{2} \mathrm{O}$-Emissionen aus dem Ap- und dem Ah-Horizont zeigten während der Ammoniumberegnung nahezu identische Verläufe (Abb. 4-7). Sie erreichten bereits nach dem Austausch von ca. einem PV durch die Beregnungslösung (Tag 145) ein Maximum. Obwohl die $\mathrm{NH}_{4}$-Applikation kontinuierlich erfolgte, verringerten sich die $\mathrm{N}_{2} \mathrm{O}$-Emissionen von maximal 19 auf ca. $3 \mathrm{~g} \mathrm{ha}^{-1} \mathrm{~d}^{-1}$ und stabilisierten sich auf diesem Niveau. Mögliche Ursachen können eine Anpassung der Nitrifikantenpopulation an die $\mathrm{NH}_{4}$-Konzentrationen oder eine Versauerung der Böden sein.

Die $\mathbf{N}_{2} \mathrm{O}-\mathrm{N}$-Emissionen (Abb. 4-8 b) während der Ammoniumdüngung betrugen ca. $0,2 \%$ des ausgebrachten Ammonium-N. Trotz kontinuierlicher Ammoniumzufuhr wurde dieser Betrag in dem betrachteten Zeitraum maßgeblich durch den $\mathrm{N}_{2} \mathrm{O}$ Emissionspeak bestimmt, wie er auch in anderen Arbeiten nach einer einmaligen Ammoniumapplikation beschrieben wurde (RACKWITZ, 1996). Der $\mathrm{N}_{2} \mathrm{O}-\mathrm{N}$-Anteil am kontinuierlich ausgebrachten $\mathrm{NH}_{4}-\mathrm{N}$ steht auch in engster Übereinstimmung mit den Ergebnissen von Arbeiten anderer Autoren, die im Freiland mit N-Düngeimpulsen arbeiteten (KelleR et al., 1988; BouwmAN, 1990; EICHNER, 1990). Diese Autoren zeigten anhand der Auswertung von Literaturdaten, daß der Median der $\mathrm{N}_{2} \mathrm{O}-\mathrm{N}$ Emissionen bei Verwendung von Ammoniumdüngern auf gut durchlüfteten Böden zwischen 0,12 und $0,15 \%$ der applizierten N-Menge lag. 


\section{$\mathrm{N}_{2}$-Emissionen}

Anhand der ${ }^{15} \mathrm{~N}$-Gasfreisetzungsmethode (Kap. 4.2.7) wurde nachgewiesen, daß von dem Ah-Horizont unter den eingestellten Versuchsbedingungen bei Nitratdüngung kein denitrifikationsbürtiger Stickstoff $\left(\mathrm{N}_{2}\right)$ emittiert wurde. Gleichzeitig konnten keine $\mathrm{N}_{2} \mathrm{O}$-Emissionen nachgewiesen werden (Kap. 4.3.3). Dieses steht im Widerspruch zu der vorangestellten N-Bilanz, nach der in Phase II Stickstoffverluste von ca. $23 \%$ des applizierten $\mathrm{N}$ vorlagen. Es wurde vermutet, daß diese Verluste neben einer biologischen Immobilisation hauptsächlich auf gasförmige N-Verluste zurückzuführen seien. Bilanzen zur Bestimmung des Denitrifikationsvermögens eines Bodens, wie sie auch von RollaNd (1995) anhand von Säulenversuchen durchgeführt wurden, sind deshalb zur Ermittlung von Denitrifikationsverlusten nicht ausreichend.

Im Ap- und Ah-Horizont sind bei einem mittleren WFPS von $82 \%$ (Ap) bzw. $77 \%$ (Ah) vermutlich alle Bereiche des Bodens über kontinuierliche Luftporen gut mit Sauerstoff versorgt, so daß ein hoher $\mathrm{O}_{2}$-Partialdruck in den Bodensäulen ein limitierender Faktor für die Denitrifikation sein dürfte. Nach STUMM und MORGAN (1981) reduzieren fakultativ anaerobe Mikroorganismen Nitrat aufgrund einer im Vergleich zur $\mathrm{O}_{2}$-Reduktion energetisch geringfügigeren Ausbeute nur bei Sauerstoffmangel. In den Bodensäulen des Ah-Horizontes dagegen dürfte die Denitrifikationsrate in den Untersuchungen zu gering ausgewiesen sein, da in den Mikrokosmen die $\mathrm{O}_{2}$-Diffusionswege von der Bodenoberfläche durch den ApHorizont hindurch, wie sie im freien Feld vorliegen, wegfallen. Es ist somit zu berücksichtigen, daß im Modell der $\mathrm{O}_{2}$-Partialdruck im Ah vermutlich unnatürlich hoch ist. Unter Feldbedingungen wäre die Wahrscheinlichkeit der Bildung anaerober Kompartimente im Ah aufgrund der vorausgegangenen $\mathrm{O}_{2}$-Zehrung im Oberboden (mikrobielle Atmung, Wurzelatmung) größer.

Da sich der Ah-Horizont sowohl hinsichtlich seiner bodenphysikalischen und -chemischen Eigenschaften (Tab. 3-1) wie auch seiner hydraulischen Eigenschaften unter Versuchsbedingungen kaum von denen des Ap-Horizontes unterschied und aus beiden Horizonten bei Nitratberegnung keine $\mathrm{N}_{2} \mathrm{O}$-Emissionen auftraten, wird unterstellt, daß es auch im Ap-Horizont zu keinen Denitrifikationsverlusten kam. Da beide Horizonte aus der Wurzelzone stammen, wird weiterhin vermutet, daß der verfügbare organische Kohlenstoff als Elektronendonator für organotrophe Denitrifikanten nicht der begrenzende Faktor ist. RYDEN et al. (1979) und BOWMAN und FOCHT (1974) weisen jedoch darauf hin, daß nur ein geringer Teil des organischen Kohlenstoffs für die organotrophen Mikroorganismen zur Verfügung steht und daß sich dementsprechend alle Prozesse, die die leicht verfügbare Kohlenstofffraktion erhöhen (Ausscheidung von Wurzelexsudaten, Einarbeitung von 
Ernterückständen, Austrocknung und Wiederbefeuchtung, Gefrieren und Auftauen), positiv auf die Denitrifikation auswirken. Diese die Denitrifikation fördernden Faktoren waren zwar unter Versuchsbedingungen nicht gegeben, jedoch spricht die gleichbleibende Bodenatmung über den gesamten Versuchszeitraum, wie sie an den Ah-Säulen nachgewiesen wurde, für eine ausreichende C-Verfügbarkeit ( Abb. 4-9).

Da eine Denitrifikation zur Erklärung der $\mathrm{NO}_{3}$-Bilanzverluste im Ah-Horizont in der Versuchsphase II ausgeschlossen wird, muß neben transportbedingten Ursachen eine mikrobielle Festlegung angenommen werden.

Im Gegensatz zum Ah konnten für die Bodensäulen des Go-Gr-Horizontes während der Inkubationsphase starke denitrifikative $\mathrm{N}_{2}$-Verluste in Höhe von $0,59 \mathrm{~kg} \mathrm{ha}^{-1} \mathrm{~d}^{-1}$ nachgewiesen werden (Tab. 4-7). Dieser Horizont emittierte während der gesamten Versuchsdauer kein $\mathrm{N}_{2} \mathrm{O}$ (Abb. 4-8); Nitrat wurde also vollständig zum molekularen Stickstoff reduziert. Es wird unterstellt, daß die ermittelten $\mathrm{N}_{2}$-Verluste der Inkubationsphase auch die Denitrifikationsverluste während der Nitratberegnung in Phase II widerspiegeln. Dieses bedeutet, daß die tägliche Nitratvorlage in Höhe von $1 \mathrm{~kg} \mathrm{NO}_{3}-\mathrm{N} \mathrm{ha}^{-1}$ zu $59 \%$ denitrifiziert wurde.

Bei der N-Bilanzierung (s.o.) wurde für die Versuchsphase II ein mittlerer N-Verlust in Höhe von $81 \%$ des zugeführten Nitratstickstoffs ermittelt. Der größte Teil dieser Verluste konnte somit der direkten ${ }^{15} \mathrm{~N}$-Gasfreisetzungsmethode auf denitrifikative Stickstoffverluste zurückgeführt werden. Bei der ermittelten Denitrifikationsrate kann es sich, wie bereits erörtert (fehlende Auflagehorizonte im Mikrokosmensystem, zusätzlicher Sauerstoffeintrag über Beregnung und Belüftung) jedoch nur um eine Mindestgröße handeln.

Mit Blick auf den Denitrifikationsprozeß im Go-Gr wird festgestellt, daß es sich bei den Denitrifikanten nicht um organotrophe Mikroorganismen handeln kann, da während der gesamten Versuchszeit kein $\mathrm{CO}_{2}$ produziert wurde. Stöchiometrische Berechnungen machen nämlich deutlich, daß bei der gemessenen Denitrifikationsrate von $0,59 \mathrm{~kg} \mathrm{~N}_{2} \mathrm{ha}^{-1} \mathrm{~d}^{-1}$ bei organotropher Denitrifikation in etwa $0,81 \mathrm{~kg} \mathrm{CO}_{2}-\mathrm{C} \mathrm{ha}^{-1} \mathrm{~d}^{-1}$ hätten emittiert werden müssen.

$\mathrm{Da}$ kein $\mathrm{CO}_{2}$ emittiert wurde, wird angenommen, daß für das hohe Denitrifikationspotential dieses Horizontes vorwiegend chemolithoautotrophe Mikroorganismen verantwortlich sind, die primäre Schwefelverbindungen, wie z.B. Pyrit und Melnikovit, oder amorphe sulfidische Verbindungen als Energiequelle nutzen (KöLlE et al., 1983; BÖTTCHER, 1992). Das Vorhandensein von Schwefel im Go-Gr-Horizont wurde anhand eines qualitativen Schwefelnachweises $(\mathrm{HCl}$, Geruchsprobe) festgestellt. Überwiegend reduzierende Bedingungen im Go-Gr wie auch die Entstehungsgeschichte dieses Horizontes (alter, unter reduzierenden Bedingungen gut konservierter Ah-Horizont mit zahlreichen eingebetteten 
Holzresten) weisen auf hohe Schwefelanreicherungen hin. SCHLIE (1989) wies im Grundwasser dieses Standortes Sulfatgehalte $\left(\mathrm{SO}_{4}{ }^{2-}\right)$ von $>200 \mathrm{mg} \mathrm{L}^{-1}$ nach, wobei die genaue Herkunft nicht geklärt werden konnte. LICKFETT (1993) wies im Saugkerzenwasser desselben Standortes $(160 \mathrm{~cm}$ u. GOF) Sulfatgehalte nach, die signifikant negativ mit den Nitratgehalten korrelierten. Auch KöLLE et al. (1990) beobachteten bei Nitratreduktion im Aquifer des Fuhrberger Feldes eine gleichzeitige Zunahme der Sulfatkonzentration und schlossen auf eine chemolithoautotrophe Denitrifikation.

In den eigenen Untersuchungen konnte im Perkolat der Bodensäulen eine Bestimmung der Sulfatkonzentrationen nicht durchgeführt werden, da diese Säulen während der Konditionierungsphase mit $\mathrm{CaSO}_{4}$-Lösung beregnet wurden. ROLLAND (1995) zeigte zudem in Brut- und in Säulenversuchen, daß die Oxidation reduzierter Schwefelverbindungen und damit verbunden das Auswaschen von Sulfat unter aeroben Bedingungen schneller abläuft als unter reduzierenden Bedingungen mit Nitrat. Daraus folgert er, daß die chemolithoautotrophe Denitrifikation langfristig nur dann eine Rolle spielen kann, wenn der Boden dauerhaft sauerstofffrei ist. Diese Situation ist zwar im Go-Gr der Versuchsflächen gegeben, in der Mikrokosmenanlage hingegen wurde Sauerstoff eingetragen.

Die eigenen Versuche zeigten, daß sich das Reduktionspotential des Go-GrHorizontes im Laufe des Versuches langsam verbrauchte, denn gegen Ende der Phase I deutete sich nach Zugabe von ca. $100 \mathrm{~kg}$ Nitrat-N ha ${ }^{-1}$ bereits ein leichter Nitratdurchbruch über das Perkolat an. Auch wurde eine von oben nach unten fortschreitende rötlich -braune Verfärbung des zuvor grau-reduzierten Horizontes beobachtet. Dies weist auf eine Oxidation des Reduktionspotentiales hin. Wenn Sulfat über die Perkolate abgeführt wird, geht der Schwefel als potentielles Reduktionsäquivalent und somit auch als Denitrifikationspotential dem System verloren.

ROLLAND (1995) wies in seinen Versuchen tägliche Schwefelabbauraten von bis zu $5 \mathrm{mg}$ pro kg trockenen Bodens nach. Nach seinen Berechnungen reicht dieses aus, um selbst seine höchsten in einem Gley gemessenen Schwefelgehalte von ca. $2 \mathrm{~g}$ $\mathrm{S} \mathrm{kg}^{-1}$ Boden in etwa 3 Jahren völlig abzubauen. WISOTZKY (1994) berichtet von Pyritabbau in Aufschüttungen aus dem rheinischen Braunkohleabbau, wo nach Exposition mit der Luft zwischen 70 und $90 \%$ des Pyrites innerhalb von 330 Tagen oxidierte.

In den ungestörten Bodensäulen des Gley-Auenbodens traten unter den eingestellten Versuchsbedingungen keine denitrifikativen $\mathrm{N}_{2} \mathrm{O}$-Emissionen auf. Infolge von Ammoniumdüngung konnten nitrifikationsbürtige $\mathrm{N}_{2} \mathrm{O}$-Emissionen 
nachgewiesen werden. Im Go-Gr-Horizont des Standortes ließ sich eine starke Denitrifikationsleistung nachweisen, die auf chemolithoautotrophe Mikroorganismen zurückzuführen war. Trotz hoher Denitrifikation wurde vom Go-Gr kein $\mathrm{N}_{2} \mathrm{O}$ emittiert. 


\section{$5 \quad$ Schlußfolgerung}

Spurengasmessungen, die in einer WRaps-WWeizen-WGerste-Fruchtfolge auf einem Gley-Auenboden über einen Zeitraum von zwei Jahren durchgeführt wurden, zeigten deutlich, daß es zu fruchtartspezifischen $\mathrm{N}_{2} \mathrm{O}$-Emissionen des Bodens kam. Beim Anbau von WRaps wurden im Versuchsmittel 1,6 $\mathrm{kg} \mathrm{N}_{2} \mathrm{O}-\mathrm{N} \mathrm{ha}^{-1} \mathrm{a}^{-1}$ emittiert, beim Anbau von WWeizen und WGerste kam es zu deutlich geringeren Emissionen in Höhe von 0,9 bzw. 0,8 $\mathrm{kg} \mathrm{N}_{2} \mathrm{O}-\mathrm{N} \mathrm{ha}^{-1} \mathrm{a}^{-1}$ (Abb. 3-12). Im Falle des Raps betrugen die Winteremissionen im Mittel ca. $70 \%$ der Gesamtjahresfreisetzungen (vgl. Tab. 37). Die höchsten Emissionsereignisse wurden bei hohem WFPS (> $80 \%$ ) oder während der Frost-Tau-Zyklen unter Stauwassereinfluß festgestellt.

Die im Vergleich höchsten $\mathrm{N}_{2} \mathrm{O}$-Emissionen nach WRaps dürften damit zu begründen sein, daß durch die leichte Mineralisierbarkeit seiner Ernterückstände (LICKFETT, 1993) (C/N 50) besonders in den Frost-Tau-Phasen Nitrat im ausreichenden Maße als Substrat für die Denitrifikation nachgeliefert wird. Außerdem dürfte eine verstärkte Mineralisation in einem durch hohen Wassergehalt schlecht durchlüfteten Boden die Geschwindigkeit der Sauerstoffzehrung und damit die Abnahme des Sauerstoffpartialdruckes bewirkt haben. LICKFETT (1993) berichtet, daß es unabhängig von der Produktionsintensität gerade nach WRaps zu erhöhten $\mathrm{N}_{\text {min }}$-Gehalten im Boden kam (> $100 \mathrm{~kg} \mathrm{ha}^{-1} 90 \mathrm{~cm}^{-1}$ ), die er auf die Mineralisation der Ernterückstände zurückführte. Hohe Bodenstickstoffgehalte tragen nach DAVIDSON (1991) zu erhöhten $\mathrm{N}_{2} \mathrm{O}$-Emissionen bei. FIRESTONE und DAVIDSON (1989) berichten von ausgeprägten denitrifikationsbürtigen $\mathrm{N}_{2} \mathrm{O}$-Emissionspeaks bei erhöhten Bodenwassergehalten. Sollen die hohen $\mathrm{N}_{2} \mathrm{O}$-Emissionen nach Raps vermieden werden, muß daher versucht werden, die Nitratgehalte im Winter nach Raps mit Hilfe einer Änderung im Bodenbearbeitungs- und Fruchtfolgekonzept (LICKFETT 1993) zu reduzieren.

Auf dem Reinshof zeigten sich in der Auftauphase des Bodens im März 1996 nach der ersten KAS-Düngung $\left(50 \% \mathrm{NO}_{3}-\mathrm{N}\right)$ z.T. deutliche $\mathrm{N}_{2} \mathrm{O}$-Emissionspeaks (vgl. Abb. 3-5 und Abb. 3-11). Um hohe $\mathrm{N}_{2} \mathrm{O}$-Emissionen im Frühjahr (=Sommerhalbjahr) zu vermeiden, sollte trotz besserer Befahrbarkeit des Ackers darauf verzichtet werden, die erste $\mathrm{N}$-Gabe auf den noch gefrorenen Boden auszubringen, da es besonders in der folgenden Auftauphase nach N-Düngung in dem von oben her auftauenden Boden in Verbindung mit stauender Nässe schnell zu extrem hohen denitrifikativen $\mathrm{N}_{2} \mathrm{O}$-Emissionen kommen kann. 
Unter Feldbedingungen ließ sich die zeitliche Variabilität der $\mathrm{N}_{2} \mathrm{O}$-Flüsse durch die als maßgebliche Einflußgrößen der $\mathrm{N}_{2} \mathrm{O}$-Emissionen geltenden Parameter Bodentemperatur, Bodenfeuchte und Bodennitratgehalt nicht bzw. nur mäßig erklären (Tab. 3-6). Dieses läßt sich mit Sicherheit auf das niedrige Emissionsniveau des Standortes zurückführen.

Der Vergleich mit anderen Versuchsstandorten („Scheyern“ und "Canstein“) zeigte, daß das $\mathrm{N}_{2} \mathrm{O}$-Emissionsniveau des Gley-Auenbodens vergleichsweise niedrig war (Abb. 3-15). LINN und DORAN (1984) weisen darauf hin, daß die Zunahme der $\mathrm{N}_{2} \mathrm{O}$ Produktion bei einem WFPS $>60 \%$ die Folge einer diffusionsbedingten Abnahme der $\mathrm{O}_{2}$-Verfügbarkeit ist. Der WFPS des Reinshof schwankte unter Feldbedingungen zwischen 92,5\% (Februar 1996) und 43,0 \% (Juni 1996). Trotz eines Anstieges des wassergefüllten Porenvolumens infolge von Niederschlägen auf $>70 \%$ konnten im Sommerhalbjahr (z.B. im Mai 1995) nur unwesentlich erhöhte $\mathrm{N}_{2} \mathrm{O}$-Emissionen nachgewiesen werden, wie sie von MOSIER et al. (1986) und RUDAZ et al. (1991) beschrieben wurden. Die Monate April bis August zeichneten sich auf dem Versuchsstandort durch gleichmäßige $\mathrm{N}_{2} \mathrm{O}$-Emissionen auf niedrigem Niveau aus.

Eine Reduzierung der ordnungsgemäßen N-Düngung um $50 \%$ führte auf dem Standort weder im Sommerhalbjahr selbst noch im Winterhalbjahr nach der Ernte zu signifikant niedrigeren $\mathrm{N}_{2} \mathrm{O}$-Emissionen (vgl. Tab. 3-7), wie sie nach EICHNER (1990) und BouWman (1994) zu erwarten gewesen wären. Dieses ist wahrscheinlich darauf zurückzuführen, daß eine Reduktion der N-Düngung zu keiner entsprechenden Reduzierung der Nitratgehalte im Boden führte, wie es z.B. auf dem Standort Scheyern der Fall war (FLESSA et al., 1998). Dort kam es während der Vegetationsperiode infolge von Niederschlagsereignissen zu deutlichen $\mathrm{N}_{2} \mathrm{O}$ Emissionen, die wahrscheinlich denitrifikativer Herkunft waren. Eine Verringerung der N-Düngung war in Scheyern eine wirkungsvolle Maßnahme zur Reduktion der direkten $\mathrm{N}_{2} \mathrm{O}$-Emissionen.

Für Methan stellte der Gley-Auenboden des Reinshof während des gesamten Versuchszeitraumes eine Senke dar. Die jährlichen Methanaufnahmeraten lagen im Versuchsmittel bei ca. 0,5 kg CH${ }_{4}-\mathrm{C}$ ha-1 (Tab. 3-9); mehr als $70 \%$ der jährlichen Oxidationsleistung erfolgte in den Sommerhalbjahren. Nach STRIEGEL (1993) ist der Haupteinflußfaktor der Methanoxidation die Höhe der Methandiffusion von der Atmosphäre zum Ort der Methanoxidierer im Boden, die Diffusionsgeschwindigkeit werde maßgeblich vom Wassergehalt des Bodens beeinflußt. Anhand der vorliegenden Ergebnisse konnten im Mittel aller Fruchtarten $40 \%$ der Variation der $\mathrm{CH}_{4}$-Aufnahme allein über den WFPS erklärt werden. Eine Abhängigkeit der Methanflußrate vom Wassergehalt des Bodens wird auch von STEUDLER et al. (1989), DöRR et al. (1993) und CZEPIEL et al. (1995) bestätigt. Mit einer jährlichen 
Aufnahmerate von ca. $0,34 \mathrm{~kg} \mathrm{CH}_{4}-\mathrm{C}$ ha $^{-1}$ unterschied sich das ungedüngte Grasland nicht signifikant von den Fruchtfolgeflächen. Die $\mathrm{CH}_{4}$-Aufnahmerate dieser Fläche wurde sehr wahrscheinlich noch durch seine landwirtschaftliche Vornutzung geprägt (vgl. DobBie et al., 1996; OJImA et al., 1993).

Im Gegensatz zu den Lachgasemissionen ließen sich bei den Methanaufnahmeraten keine fruchtartspezifischen Flußraten erkennen. Auch eine Reduktion der NDüngung bewirkte keine Erhöhung der Methanoxidation. Zu dem gleichen Ergebnis kam auch BORKEN (1996) auf gedüngten Buchenflächen. HüTSCH et al. (1994) und STEUDLER et al. (1996) berichten, daß im Vergleich zur Landnutzungsänderung der unmittelbare $\mathrm{N}$-Düngereffekt vergleichsweise klein ist. Im Vergleich zu den Standorten Scheyern und Canstein zeigte der Reinshof eine fast doppelt so hohe Oxidationsleistung (FLESSA et al., 1998), was bedeutet, daß sich dieser Standort durch eine vergleichsweise gute Gaspermeabilität des Bodens auszeichnet.

Die Laborversuche wiesen im Hinblick auf $\mathrm{N}_{2} \mathrm{O}$-Emissionen verschiedener Bodenhorizonte auf einige Besonderheiten des Standortes hin, denn bei ständiger Nitratzufuhr kam es sowohl im Ap- als auch im Ah-Horizont bei einem mittleren WFPS von 82 (Ap) bzw. $77 \%$ (Ah) zu keinen (denitrifikativen) $\mathrm{N}_{2} \mathrm{O}$-Verlusten (Kap. 4.4). Dieses könnte damit zu erklären sein, daß sich der Boden trotz feinkörniger Textur durch eine starke Aggregierung (Polyedergefüge) und somit wahrscheinlich selbst bei hohen Wassergehalten immer noch durch eine ausreichende $\mathrm{O}_{2}$-Diffusion in den Boden hinein auszeichnet. Auch die im Vergleich zu den Standorten Scheyern und Canstein hohe annuelle $\mathrm{CH}_{4}$-Oxidationsleistung dieses Ackerstandortes (vgl. Abb. 3-17) ließ bereits eine gute Gaspermeabilität des Bodens vermuten (s.o.).

Die $\mathrm{N}_{2} \mathrm{O}$-Emissionen, wie sie sich unter den statischen Bedingungen im Labor zeigen, sind jedoch nicht uneingeschränkt auf Freilandbedingungen zu übertragen. Allein die konstant hohen Wassergehalte des Bodens unter Laborbedingungen können zu einem Rückgang denitrifikativer $\mathrm{N}_{2} \mathrm{O}$-Emissionen führen (vgl. MULVANEY und KURTZ, 1984). Da im Vergleich zu Freilandbedingungen in den Bodensäulen sowohl die fehlende Wurzelatmung als auch die fehlende Mineralisation von kürzlich eingearbeitetem Pflanzenmaterial zu keinem zusätzlichen $\mathrm{O}_{2}$-Verbrauch führen, dürfte unter sonst gleichen Bedingungen der $\mathrm{O}_{2}$-Partialdruck im Boden in vitro hoch sein. Außerdem kommt es beim Ah aufgrund des fehlenden Auflagehorizontes infolge einer Verkürzung der Diffusionswege für atmosphärische Luft unter Laborbedingungen zu vergleichsweise hohen Sauerstoffeinträgen.

$\mathrm{Da}$ es jedoch unter Laborbedingungen bei kontinuierlicher Nitratzufuhr im ApHorizont selbst bei einem mittleren WFPS von $82 \%$ zu keinen $\mathrm{N}_{2} \mathrm{O}$-Emissionen kam, kann davon ausgegangen werden, daß die unter Freilandbedingungen bei einem 
WFPS $<80 \%$ auftretenden $\mathrm{N}_{2} \mathrm{O}$-Emissionen zum überwiegenden Teil nitrifikationsbürtig waren. Die Tatsache, daß es sowohl im Ap-Horizont als auch im Ah-Horizont unter Laborbedingungen erst nach kontinuierlicher Ammoniumzufuhr zu maximalen $\mathrm{N}_{2} \mathrm{O}$-Emissionen zwischen 16 und $19 \mathrm{~g} \mathrm{~N}_{2} \mathrm{O}-\mathrm{N} \mathrm{ha}^{-1} \mathrm{~d}^{-1}$ kam (Abb. 4-7), zeigt die Bedeutung der Nitrifikation für $\mathrm{N}_{2} \mathrm{O}$-Emissionen dieses Standortes. Selbst im Anschluß an den beschriebenen Emissionspeak nach Beginn der Ammoniumzugabe betrugen die täglichen $\mathrm{N}_{2} \mathrm{O}-\mathrm{N}$-Emissionen noch ca. $3 \mathrm{~g} \mathrm{ha}^{-1}$, was einer jährlichen Emission von ca. $1 \mathrm{~kg} \mathrm{~N}_{2} \mathrm{O}-\mathrm{N}^{-1}$ entspricht. Die tatsächlich in der Fruchtfolge gemessenen jährlichen Gesamtemissionen lagen zwischen 0,57 und $1,8 \mathrm{~kg} \mathrm{~N}_{2} \mathrm{O}-\mathrm{N} \mathrm{ha}^{-1}$ (vgl. Tab. 3-7). Dieses ist ein Hinweis darauf, daß potentiell zumindest ein großer Teil der $\mathrm{N}_{2} \mathrm{O}$-Emissionen dieses Standortes nitrifikationsbürtig sein kann. Auch KÖBRICH (1995) kommt zu dem Ergebnis, daß für Versuchsflächen südlich von Braunschweig (Parabraunerde aus Löß mit Tongehalten bis $22 \%$ ) die Nitrifikation mit einem erheblichen Anteil zur $\mathrm{N}_{2} \mathrm{O}$-Freisetzung beiträgt. Die Bodenschicht aus einer Tiefe von $0-10 \mathrm{~cm}$ unter GOF wird als hauptverantwortlich für die nitrifikationsbürtige $\mathrm{N}_{2} \mathrm{O}$-Freisetzung angesehen (SCHNEIDER, 1991).

Auf dem Reinshof dürften deshalb auch die z.T. leicht erhöhten $\mathrm{N}_{2} \mathrm{O}$-Emissionen nach KAS-Düngung (50\% $\left.\mathrm{NH}_{4}-\mathrm{N}\right)$ im April und Mai (Abb. 3-10 und 3-11) überwiegend nitrifikativer Herkunft sein. Auch SKIBA et al. (1993) fanden in sandigen Lehmböden höhere nitrifikationsbürtige $\mathrm{N}_{2} \mathrm{O}$-Flußraten nach der Düngung.

Auch die leicht angestiegenen $\mathrm{N}_{2} \mathrm{O}$-Emissionen direkt nach der Ernte des WRaps im Juli/August dürften auf eine erhöhte Nitrifikation zurückzuführen sein. Gefördert durch eine langsam einsetzende Wiederbefeuchtung des Bodens bei gleichzeitig guter Durchlüftung (lockernde Bodenbearbeitung) wird durch die Einarbeitung der Erntereste in Abhängigkeit von deren Qualität und Quantität die N-Mineralisation gesteigert. Dieses wirkt sich unmittelbar auf einen Anstieg der Nitrifikationsleistung des Bodens aus, während die Denitrifikation direkt nach der Ernte bei einem WFPS von meistens $<70 \%$ im Ap-Horizont des Reinshof nach den Erkenntnissen aus den Laborversuchen eher eine untergeordnete Rolle spielen dürfte. Die Leistungsfähigkeit der Nitrifikanten sowohl im Ap als auch im Ah reichte selbst bei einem WFPS von ca. $80 \%$ bei gleichzeitiger Beregnung von $4 \mathrm{~mm} \mathrm{~d}^{-1}$ noch aus, um eine tägliche Ammoniumzufuhr von $4 \mathrm{~kg} \mathrm{ha}^{-1}$ vollständig zu nitrifizieren.

Erst wassergefüllte Porenvolumina $>80 \%$ (Abb. 3-6 und 3-7) bzw. aufgestautes Wasser nach der Schneeschmelze, wie es von Dezember bis März auf dem Standort vorkam, dürfte zu relativem Sauerstoffmangel im Boden geführt haben, so daß es infolgedessen $z u$ einem deutlichen Anstieg von $\mathrm{N}_{2} \mathrm{O}$-Emissionen denitrifikativer Herkunft kam (Abb. 3-10 und 3-11). Ein Hinweis auf denitrifikationsbürtige $\mathrm{N}_{2} \mathrm{O}$ - 
Emissionen des Reinshof ist, daß sich nur für die Winterhalbjahre (alle Fruchtarten der Fruchtfolge) ein signifikant positiver Zusammenhang zwischen den mittleren Nitratgehalten des Bodens und den halbjährlich kumulierten $\mathrm{N}_{2} \mathrm{O}$-Emissionen nachweisen ließ (Abb. 3-16) (vgl. MosiER et al., 1993). Die Vermutung einer denitrifikativen Herkunft der $\mathrm{N}_{2} \mathrm{O}$-Emissionen wird zudem dadurch gestützt, daß es gerade in diesen Monaten zu sprunghaften, innerhalb kurzer Zeit um mehrere Größenordnungen ansteigenden Emissionsraten kam. Die Nitrifikation hingegen weist in den meisten terrestrischen Böden keine entsprechend großen Aktivitätssprünge auf (FIRESTONE und DAVIDSON, 1989).

Die direkte Messung denitrifikativer $\mathrm{N}_{2}$-Emissionen an ungestörten Bodensäulen im Labor ergab, daß es im Ah-Horizont nach kontinuierlicher Nitratzufuhr bei einem mittleren WFPS von $77 \%$ nicht zu denitrifikativen Stickstoffverlusten kam. Aufgrund ähnlicher bodenphysikalischer und bodenchemischer Eigenschaften des ApHorizontes wurde davon ausgegangen, daß auch dieser Horizont ebenfalls keine denitrifikativen $\mathrm{N}_{2}$-Verluste zeigte (Kap 4.4).

Anhand der Feldversuche konnte nicht geklärt werden, ob in tieferen Bodenhorizonten des Reinshof $\mathrm{N}_{2} \mathrm{O}$ gebildet wurde und welche Bildungsprozesse hierfür eine Rolle spielten. LICKFETT (1993) vermutete, daß es in Reinshof zwischen 80 und $160 \mathrm{~cm}$ unter GOF zu erheblichen denitrifikativen Stickstoffverlusten $\left(\mathrm{N}_{2}\right.$, $\mathrm{N}_{2} \mathrm{O}$, NO) kam, da er in diesem Bereich einen starken Rückgang der Nitratkonzentrationen in der Bodenlösung feststellte. Mit Hilfe der ungestörten Bodensäulen aus 140 - $165 \mathrm{~cm}$ unter GOF (Go-Gr-Horizont) konnte in dieser Arbeit nachgewiesen werden, daß sich nach Nitratdüngung die denitrifikativen $\mathrm{N}_{2}$-Verluste auf $0,59 \mathrm{~kg} \mathrm{ha}^{-1} \mathrm{~d}^{-1}$ beliefen, was einer Denitrifikationsleistung von $59 \%$ des zuvor täglich applizierten Nitratstickstoffs entsprach. Denitrifikative NO- und $\mathrm{N}_{2} \mathrm{O}-$ Verluste traten in diesem Horizont hingegen nicht auf, d.h. sämtliches Nitrat wurde bis zum molekularen Stickstoff reduziert. Da sich gleichzeitig keine $\mathrm{CO}_{2}$-Emission zeigte, war die Denitrifikation vermutlich auf die mikrobielle Oxidation reduzierter Schwefelverbindungen zurückzuführen (Kap. 4.4). Trotz starker Denitrifikationsleistung dieser Bodensäulen wurde jedoch kein $\mathrm{N}_{2} \mathrm{O}$ emittiert. Bei einem WFPS von $93 \%$, welcher auch die meist wassergesättigten Verhältnisse im Felde widerspiegelt, scheint bei hoher Denitrifikationsrate die Gasdiffusion stark behindert zu sein, wodurch das $\mathrm{N}_{2} \mathrm{O} / \mathrm{N}_{2}$-Verhältnis stark abnehmen kann (DAVIDSON, 1991). Damit scheidet dieser überwiegend reduktive Horizont des Reinshof als Quelle von denitrifikativen $\mathrm{N}_{2} \mathrm{O}$-Emissionen aus, emittiertes $\mathrm{N}_{2} \mathrm{O}$ muß also in den darüberliegenden Bodenhorizonten produziert worden sein.

In Feldversuchen auf dem Standort Reinshof konnte schon LICKFETT (1993) nachweisen, daß es trotz z.T. hoher N-Bilanzüberschüsse im Winterraps zu keinen 
bzw. nur zu geringen Nitratausträgen ins Grundwasser kam. Nach den Erkenntnissen der Laborversuche dürfte dieses ebenfalls auf die hohe Denitrifikationsleistung des Go-Gr-Horizontes zurückzuführen sein (Kap. 4.4). Die Gefahr einer Stickstoffbelastung angrenzender Naturräume über den Aquifer und der damit verbundenen Möglichkeit der Erhöhung indirekter $\mathrm{N}_{2} \mathrm{O}-$ Emissionen (DUXBURY et al., 1993) wird dadurch weitgehend minimiert.

Die Laborversuche zeigten jedoch, daß das Reduktionspotential bei einem massiven Eintrag von Oxidationsmitteln $\left(\mathrm{NO}_{3}, \mathrm{O}_{2}\right)$ vermutlich verbraucht wird. Wird Schwefel in der wasserlöslichen, oxidierten Form (Sulfat) über das Sickerwasser abgeführt, steht dieser als Reduktionsmittel nicht mehr zur Verfügung. Ist das Reduktionspotential des Go-Gr-Horizontes weitgehend erschöpft, besteht die Gefahr, daß es auf dem Standort aufgrund der schwachen Denitrifikationsleistung im Oberboden zum „Durchbruch“ von Nitratfrachten kommt. Abgesehen von der Eutrophierung angrenzender Naturräume hätte dieses zur Folge, daß es dort unter Umständen zu erhöhten $\mathrm{N}_{2} \mathrm{O}$-Emissionen (indirekte $\mathrm{N}_{2} \mathrm{O}$-Emissionen) käme, die der jeweils angebauten Frucht auf dem Reinshof zugeschrieben werden müßten. Besonders hoch wären dann die indirekten $\mathrm{N}_{2} \mathrm{O}$-Emissionen für den Winterraps anzusetzen. Ein alternatives Anbaukonzept, wie es von LICKFETT et al. (1993) im Zusammenhang mit einer möglichen Minimierung der Nitratauswaschungsgefahr nach WRaps vorgeschlagen wurde, könnte neben einer unmittelbaren Reduktion der direkten $\mathrm{N}_{2} \mathrm{O}$-Emissionen auch dazu beitragen, daß das „natürliche“ Reduktionspotential im Go-Gr-Horizont dieses Standortes möglichst lange erhalten bleibt.

Allgemeingültige Aussagen bezüglich der Klimarelevanz der Produktion von Winterraps als Ersatz von Rapsölmethylester können anhand der Spurengasmessungen auf dem Reinshof nur vorbehaltlich der oben genannten Besonderheiten des Standortes hinsichtlich der $\mathrm{N}_{2} \mathrm{O}$-Emissionen gemacht werden. Vor allen Dingen das damit verbundene niedrige $\mathrm{N}_{2} \mathrm{O}$-Emissionsniveau ( $\mathrm{vgl}$. Abb. 3-15) dürfte zu einer Unterschätzung der beim Anbau von WRaps typischen Feldemissionen führen. Die aufgenommenen Methanmengen in der Fruchtfolge ließen erkennen, daß diese im Vergleich $\mathrm{zu}$ den $\mathrm{N}_{2} \mathrm{O}$-Emissionen für die Treibhausbilanz der Produktion unbedeutend waren.

Der Anbau von WRaps trägt im Vergleich zu den Fruchtarten WWeizen und WGerste in höherem Maß zur Verstärkung des Treibhauseffektes bei. Grund hierfür waren die erhöhten fruchtspezifischen direkten $\mathrm{N}_{2} \mathrm{O}$-Feldemissionen, deren Anteil an der gesamten Atmosphärenbelastung beim Anbau von WRaps zwischen $29 \%(\mathrm{~N}$ Düngung It. Offizialberatung) und $39 \%$ (um $50 \%$ reduzierte N-Düngung) lag. 
Trotz des standortspezifisch geringen Emissionsniveaus machten die Emissionen biologischen Ursprungs (direkte und indirekte Lachgasemissionen im Feld) im Versuchsmittel jedoch über $50 \%$ der gesamten klimawirksamen Atmosphärenbelastung beim Anbau von WRaps aus (Tab. 3-11). Bei Betrachtung des gesamten Lebensweges der RME-Produktion reduzieren allein die $\mathrm{N}_{2} \mathrm{O}$-Emissionen biologischen Ursprungs das gesamte in $\mathrm{CO}_{2}$-Äquivalenten ausgedrückte Einsparungspotential des RME um ein Drittel (vgl. Kap. 3.4.3). Da unter Berücksichtigung des gesamten Lebensweges der Rapsölmethylester-Produktion auf dem Reinshof das $\mathrm{CO}_{2}$-Einsparungspotential des RME völlig zunichte gemacht würde, käme es auf Standorten mit einem deutlich höheren Niveau der $\mathrm{N}_{2} \mathrm{O}$ Feldemissionen (vgl. Abb. 3-15) beim Anbau von Winterraps als Ersatz von Dieselkraftstoff sogar zu einer zusätzlichen Belastung der Erdatmosphäre. 


\section{$6 \quad$ Zusammenfassung}

In Deutschland wurden 1997 ca. 914.000 ha Winterraps angebaut. Hiervon ist ein großer Teil für die Rapsölmethylestererzeugung (RME) als Ersatz von Dieselkraftstoff bestimmt, um u.a. die anthropogen bedingten Emissionen treibhauswirksamer Gase $\left(\mathrm{CO}_{2}, \mathrm{~N}_{2} \mathrm{O}, \mathrm{CH}_{4}\right)$ aus der Verbrennung fossiler Energieträger zu verringern. Weitgehend unbekannt ist, ob es durch den Anbau von WRaps zu zusätzlichen Emissionen von klimarelevanten Spurengasen kommt, durch die eine Entlastung der Erdatmosphäre minimiert würde. WRaps hinterläßt nach seiner Ernte auch bei ordnungsgemäßer Düngung erhebliche Mengen an mineralischem Stickstoff im Boden, so daß im Vergleich zu anderen Fruchtarten ein großer Substratpool für eine nitrifikative oder denitrifikative $\mathrm{N}_{2} \mathrm{O}$-Bildung im Boden vorhanden ist. Im Rahmen der vorliegenden Arbeit wurden deshalb die Flüsse der Spurengase $\mathrm{N}_{2} \mathrm{O}$ und $\mathrm{CH}_{4}$ in der Fruchtfolge Winterraps-Winterweizen-Wintergerste bei differenzierter N-Düngung auf einem Gley-Auenboden $2 \mathrm{~km}$ südlich von Göttingen über einen Zeitraum von 2 Jahren in wöchentlichen Abständen gemessen und in Bezug zu einigen Einflußfaktoren gesetzt, die nach Literaturangaben auf die Spurengasflüsse des Bodens wirken.

Mit Hilfe von Laborversuchen (Mikrokosmen) wurde darüber hinaus der Einfluß des Ammoniums und des Nitrats auf die $\mathrm{N}_{2} \mathrm{O}$-Emissionen des Standortes untersucht. Hierbei wurde die Bedeutung der Nitrifikation und der Denitrifikation in ungestörten Bodensäulen des Ap-, Ah- sowie des Go-Gr-Horizontes (nur Denitrifikation) abgeleitet. Zusätzlich wurde geklärt, welche der Bodenhorizonte für die Entstehung von $\mathrm{N}_{2} \mathrm{O}$ in Betracht kommen. Außerdem wurde die Denitrifikationsleistung im Ahund Go-Gr-Horizont mit Hilfe der ${ }^{15} \mathrm{~N}$-Gasfreisetzungsmethode direkt bestimmt.

Aufgrund der vorliegenden Ergebnisse wurde abschließend eine ökologische Bewertung des Rapsanbaus mit Blick auf $\mathrm{N}_{2} \mathrm{O}$ und $\mathrm{CH}_{4}$ durchgeführt.

Der Versuchsstandort Reinshof wies vergleichsweise niedrige $\mathrm{N}_{2} \mathrm{O}$-Emissionsraten auf. Im Versuchsmittel wurden in der Fruchtfolge jährlich zwischen 0,72 und 1,59 kg $\mathrm{N}_{2} \mathrm{O}-\mathrm{N}$ ha ${ }^{-1}$ emittiert; im Vergleich dazu wies eine ungedüngte Grasfläche des Standortes eine mittlere $\mathrm{N}_{2} \mathrm{O}$-Emission von $0,34 \mathrm{~kg} \mathrm{~N}_{2} \mathrm{O}-\mathrm{N} \mathrm{ha}^{-1} \mathrm{a}^{-1}$ auf.

Die Gesamt $-\mathrm{N}_{2} \mathrm{O}$-Emissionen der drei Fruchtarten waren in den Winterhalbjahren (1. September bis 28./29. Februar) nach der jeweiligen Ernte positiv mit den mittleren Nitrat-Gehalten des Bodens $(0-30 \mathrm{~cm})$ korreliert. Besonders deutlich zeigten sich erhöhte Bodennitratgehalte im Winterhalbjahr nach Raps, die in erster Linie auf die Mineralisation der Ernte- und Wurzelrückstände zurückgeführt wurden. 
Infolgedessen kam es nach Winterraps auch zu den höchsten $\mathrm{N}_{2} \mathrm{O}$-Emissionen. Die Winteremissionen beim Anbau von WRaps machten im Mittel ca. $70 \%$ der Gesamtjahresemissionen aus.

Eine Halbierung der mineralischen N-Düngung (KAS) auf durchschnittlich $76 \mathrm{~kg}$ $\mathrm{ha}^{-1} \mathrm{a}^{-1}$ wirkte sich in der gesamten Fruchtfolge nicht auf die $\mathrm{N}_{2} \mathrm{O}$ Gesamtjahresflüsse aus.

Die zeitliche Variabilität der $\mathrm{N}_{2} \mathrm{O}$-Flüsse im Feld ließ sich anhand der als maßgebliche Steuergrößen der $\mathrm{N}_{2} \mathrm{O}$-Emissionen geltenden Parameter Bodentemperatur, Bodenfeuchte und Bodennitratgehalt nicht bzw. nur in Ansätzen erklären. Hierfür wird das vergleichsweise niedrige $\mathrm{N}_{2} \mathrm{O}$-Emissionsniveau des Standortes verantwortlich gemacht.

Die Methanaufnahmeraten in der Fruchtfolge des Reinshof schwankten zwischen 0,58 und $0,45 \mathrm{~kg} \mathrm{CH}_{4}-\mathrm{C} \mathrm{ha}{ }^{-1} \mathrm{a}^{-1}$, auf der Grasfläche beliefen sie sich auf $0,36 \mathrm{~kg}$ $\mathrm{CH}_{4}-\mathrm{C} \mathrm{ha}^{-1} \mathrm{a}^{-1}$.

Die Variation der Methanoxidation konnte im Versuchsmittel zu ca. $40 \%$ über die Dynamik des wassergefüllten Porenvolumens des Bodens erklärt werden. Die kumulierten, durch den Boden aufgenommenen Methanmengen korrelierten sowohl in den Sommer- als auch in den Winterhalbjahren deutlich negativ mit dem wassergefüllten Porenvolumen des Bodens.

Fruchtartspezifische Effekte ließen sich hingegen für die Methanoxidation nicht nachweisen. Auch eine Reduzierung der N-Düngung um $50 \%$ führte in der gesamten Fruchtfolge zu keiner Reduzierung der $\mathrm{CH}_{4}$-Gesamtjahresflüsse.

Die Laboruntersuchungen an ungestörten Bodensäulen des Gley-Auenbodens zeigten, daß es bei einem WFPS von ca. $80 \%$ im Ap- und Ah-Horizont bei Nitratapplikation zu keinen gasförmigen $\mathrm{N}$-Verlusten kam. Erst Ammoniumapplikation führte zu deutlichen $\mathrm{N}_{2} \mathrm{O}$-Emissionen. Hieraus wurde geschlossen, daß diese $\mathrm{N}_{2} \mathrm{O}$-Emissionen nitrifikationsbürtig waren. Es wurden maximale $\mathrm{N}_{2} \mathrm{O}$ Emissionen von umgerechnet bis zu $19 \mathrm{~g} \mathrm{~N}_{2} \mathrm{O}-\mathrm{N} \mathrm{ha}^{-1} \mathrm{~d}^{-1}$ gefunden, die sich nach längerer Ammoniumzugabe bei ca. $3 \mathrm{~g} \mathrm{~N}_{2} \mathrm{O}-\mathrm{N} \mathrm{ha}^{-1} \mathrm{~d}^{-1}$ stabilisierten. Letzteres entspricht einer jährlichen $\mathrm{N}_{2} \mathrm{O}-\mathrm{N}$-Emission in Höhe von über einem Kilogramm pro Hektar und Jahr und liegt somit in einem Bereich, der auch in den Feldversuchen nachgewiesen wurde.

Im Go-Gr-Horizont (140-165 cm unter GOF) des Reinshof kam es bei kontinuierlicher Nitratzufuhr zu keinen $\mathrm{N}_{2} \mathrm{O}$-Emissionen, jedoch konnten hier nach 
Applikation von ${ }^{15} \mathrm{~N}$-markiertem Nitrat hohe denitrifikative $\mathrm{N}_{2}$-Verluste direkt nachgewiesen werden.

Sowohl die Tatsache, daß aus diesem Horizont bei überwiegend reduktiven Verhältnissen kein $\mathrm{CO}_{2}$ emittiert wurde, als auch der qualitative Nachweis von Schwefel lassen vermuten, daß es sich um eine autotrophe Denitrifikation handelt. Der allmähliche „Durchbruch“ von Nitrat in den Bodensäulen des Go-Gr-Horizontes im Verlaufe des Versuches wies darauf hin, daß sich das Reduktionspotential aufgrund des Eintrages von Oxidationsmitteln in Form von Nitrat und Sauerstoff langsam verbrauchte.

Aus den Untersuchungen wird deutlich, daß der Anbau von WRaps im Vergleich zu den Fruchtarten WWeizen und WGerste in höherem Maß zur Verstärkung des Treibhauseffektes beiträgt. Grund hierfür waren die erhöhten fruchtartspezifischen direkten $\mathrm{N}_{2} \mathrm{O}$-Feldemissionen, deren Anteil an der gesamten Atmosphärenbelastung beim Anbau von WRaps zwischen $29 \%$ (N-Düngung lt. Offizialberatung) und $39 \%$ (um $50 \%$ reduzierte $\mathrm{N}$-Düngung) lag.

Bei Betrachtung des gesamten Lebensweges der RME-Produktion reduzieren allein die $\mathrm{N}_{2} \mathrm{O}$-Emissionen biologischen Ursprungs (direkte und indirekte Lachgasemissionen im Feld) das gesamte in $\mathrm{CO}_{2}$-Äquivalenten ausgedrückte Einsparungspotential des RME um ein Drittel. Die klimarelevanten Emissionen energetischen Ursprunges beim Anbau von WRaps (Kraftstoff-, Düngemittel- und Pflanzenschutzmittelbereitstellung) würden dieses um ein weiteres Drittel reduzieren. Die weitere Berücksichtigung von Emissionen bei der industriellen RME-Produktion sowie bei der RME-Verbrennung $\left(\mathrm{N}_{2} \mathrm{O}\right.$ und $\left.\mathrm{CH}_{4}\right)$ macht das $\mathrm{CO}_{2}{ }^{-}$ Einsparungspotential des nachwachsenden Rohstoffes WRaps vollständig zunichte. 


\section{$7 \quad$ Literaturverzeichnis}

Abou-SeAdA, M.N.I. und J.C.G. OtTow (1995): Effect of increasing oxygen concentration on total denitrification and nitrous oxide release from soil by different bacteria. Biol. Fert. Soils 1, 31-38.

AdAmSEN, A.P.S und G.M. King (1993): Methane consumption in temperate and subarctic forest soils - rates, vertical zonation, and responses to water and nitrogen. Appl. Environ. Microbiol. 59, 485-490.

Ambus, P. und S. Christensen (1995): Spatial and seasonal nitrous oxide and methane fluxes in danish forest-, grassland-, and agroecosystems. J. Environ. Qual. 24, 993-1001.

ANDERSON, I.C. und J.S. LEVINE (1986): Relative rates of nitric oxide production by nitrifiers, denitrifiers and nitrate respirers. Appl. Environ. Microbiol. 51, 938-945.

ARDAKANI, M.S., M.S. BeLSER und A.D. McLAREN (1975): Reduction of nitrate in a soil column during continuous flow. Soil Sci. Soc. Am. Proc. 39, 290-294.

AulakH, M.S., D.A. ReNNIE und E.A. Paul (1982): Gaseous nitrogen losses from cropped and summer-fallowed soils. Can. J. Soil Sci. 62, 187-196.

AulAKH, M.S., D.A. RENNIE und E.A. PAUL (1984): Gaseous nitrogen losses from soils under zero-till as compared with conventional-till management systems. J. Environ. Qual. 13, 130-136.

AULAKH, M.S., J.W. DORAN und A.R. MOSIER (1992): Soil denitrification - significance, measurement, and effect of management. Adv. Soil Sci. 18, $1-57$.

BADR, O. und S.D. Probert (1992): Sources of atmospheric nitrous oxide. J. Appl. Energy 42: 129-176.

BARTHOLOMEW, W.V. und E. CLARK (1950): Nitrogen transformation in soil in relation to the rhizosphere microflora. $4^{\text {th }}$ Int. Congr. Soil Sci. Soc. Amsterdam.

BATJES, N.H. (1992): Nitrous oxide. pp. 49-86. In: World inventory of soil emissions. International Soil Reference and Information Centre, N.H. Batjes and E.M. Bridges, The Netherlands.

BATJES, N.H. und E.M. BRIDGES (1992): World inventory of soil emissions. International Soil Reference and Information Centre, Wageningen, Netherlands. 
BECKER, K.W. (1991): Nitratsteuerung in Wassereinzugsgebieten: Regionale Unterschiede in der $\mathrm{N}$-Belastung der Agrar-Ökotope aufgrund der standortabhängigen Nutzungsweisen und Produktionsgewohnheiten. Mitt. Dt. Bodenkundl. Ges., 66/2, 907-910.

BedARD, C. und R. KNowles (1989): Physiology, biochemistry, and specific inhibitors of $\mathrm{CH}_{4}, \mathrm{NH}_{4}$, and $\mathrm{CO}$ oxidation by methanothrophs and nitrifiers. Microbiol. Rev. 53, 68-84.

Beese, F. und P.R. van der Ploeg (1979): Simulation des Anionen-Transports in ungestörten Bodensäulen unter stationären Fließbedingungen. $Z$. Pflanzenernähr. Bodenk. 142, 69-85.

BeEse, F. und E. MATZneR (1986): Langzeitperspektiven vermehrten Stickstoffeintrages in Waldökosystemen: Droht Eutrophierung? Ber. Forschungsz. Waldökosysteme Reihe B, Bd. 3, 182-204.

BEESE, F. (1993): Gasförmige Stickstoffverbindungen. Studienprogramm Landwirtschaft, Band 1. Hrsg. Enquete-Kommission „Schutz der Erdatmosphäre" des deutschen Bundestages. Economica Verlag Bonn.

BENDER M. und R. ConRAD (1992): Kinetics of $\mathrm{CH}_{4}$ oxidation in oxic soils exposed to ambient air or high $\mathrm{CH}_{4}$ mixing ratios. FEMS Microbiol. Ecol. 101, 261-270.

Benkiser, G., K.-M. Syring, G. Gaus, K. Haider und D. Sauerbeck (1987): Einfluß verschiedener Bodenvariablen auf die Denitrifikation landwirtschaftlich genutzter Flächen. VDLUFA-Schriftenreihe, Kongressband 23, 937-952.

BLACKMER M.K., J.M. BREMNER und E.L. SCHMIDT (1980): Production of nitrous oxide by ammonia-oxidizing chemoautotrophic microorganisms in soil. Appl. Environ. Microbiol. 40, 1060-1066.

BLACKMER M.K. und J.M. BREMNER (1981): Terrestrial nitrification as a source of atmospheric nitrous oxide. In: Delwiche CC (ed.): Denitrification, nitrification and atmospheric $\mathrm{N}_{2} \mathrm{O}$. pp. 164-170, John Wiley and Sons Ltd., Chichester.

Blackmer, A.M. und M.E. CerRato (1986): Soil properties affecting formation of nitric oxide by chemical reactions of nitrite. Soil Sci. Soc. A. J. 50, 12151218.

BLAKE, D.R. und F.S. ROWLAND (1988): Continuing worldwide increase in tropospheric methane, 1978-1987. Science 239, 1129-1131.

BötTCHER, J. (1992): Stoffanlieferung an das Grundwasser bei Sandböden und Stoffumsetzungen in einem Lockersteins-Aquifer. Habilitationsschrift, Hannover, S. 118. 
BORKEN, W. (1996): Methan-Aufnahme und Kohlendioxid-Freisetzung von Waldböden. Diss. Göttingen, Berichte des Forschungszentrums Waldökosysteme, Reihe A, Bd. 137.

BORKEN, W. und R. BRUMmE (1997): Liming practice in temperate forest ecosystems and the effects on $\mathrm{CO}_{2}, \mathrm{~N}_{2} \mathrm{O}$ and $\mathrm{CH}_{4}$-fluxes. Soil Use and Management 13 , 251-257.

BouWman, A.F. (1990): Exchange of greenhouse gases between terrestrial ecosystems and the atmosphere. Pp. 61-127 in A.F. Bouwman (ed.): Soils and the greenhouse effect. John Wiley and sons Ltd., Chichester.

Bouwman, A.F. (1994): Direct emissions of nitrous oxide from agricultural soils. Report No. 773004004, National Institute of Public Health and Environmental Protection, Bilthoven, The Netherlands.

Bowman, R.A. und D.D. FOCHT (1974): The influence of glucose and nitrate concentrations upon denitrification rates in sandy soils. Soil Biol. Biochem. 6, 297-301.

Bowden, R.D., M.S. Castro, J.M. Melillo, P.A. Steudler und J.D. Aber (1993): Fluxes of greenhouse gases between soils and the atmosphere in a temperate forest following a simulated hurricane blowdown. Biogeochemistry 21, 61-71.

BREMNER, J.M. und A.M. BLACKMER (1981): Terrestrial nitrification as a source of atmospheric nitrous oxide. pp. 151-170 in C.C. Delwiche (ed.): Denitrification, nitrification and atmospheric $\mathrm{N}_{2} \mathrm{O}$. John Wiley and sons Ltd., Chichester.

Brumme, R, und G. AdEN (1995): The use of ${ }^{15} \mathrm{~N}$ to study the nitrogen turnover in soils. Methods in Applied Soil Microbiology and Biochemistry, pp. 246-250.

BuRESH, R.J., M.I. SAMSON und S.K. DeDatTA (1993): Quantification of denitrification in flooded soils as affected by rice establishment method. Soil Biol. Biochem. $25,7,843-848$.

BURFORD, J.R. und J.M. BREMNER (1975): Relationships between the denitrification capacities of soil and total, water-soluble and readily decomposable soil organic matter. Soil Biol. Biochem. 7, 389-394.

Castro, M.S., P.A. Steudler und J.M. Melillo (1995): Factors controlling atmospheric methane consumption by temperate forest soils. Global Biogeochem. Cycles. 9(1), 1-10.

CATES, R.L. und D.R. KEENEY (1987): Nitrous oxide production throughout the year from fertilized and manured maize fields. J. Environ. Qual. 16(4), 443-447. 
ChRISTENSEN, S. (1985): Denitrification in a sandy loam soil as influenced by climatic and soil conditions. Tidsskr. Planteavl. 89, 351-365.

Cicerone R.J. und R.S. OREMLAND (1988): Biogeochemical aspects of atmospheric methane. Global Biogeochemical Cycles 2: 299-327.

ConRAd, R. und W. SeILER (1980): Field measurements of the loss of fertilizer nitrogen into the atmosphere as nitrous oxide. Atm. Environ. 14, 555-558.

ConRad, R. W., SeILER und G. Bunse (1983): Factors influencing the loss of fertilizer nitrogen into the atmosphere as nitrous oxide. J. Geophys. Res. 88, 67096718.

CONRAD, R. und F. RothFuss (1991): Methane oxidation in the soil surface layer of a flooded rice field and the effect of ammonium. Biol. Fertil. Soils 12, 28-32.

CRiBBS, W.J. und H.A. Mills (1979): Influence of nitrapyrin on the evolution of nitrogen oxide $\left(\mathrm{N}_{2} \mathrm{O}\right)$ from an organic medium with and without plants. Commun. Soil Sci. Plant Anal. 10, 785-794.

CRILL, P.M. (1991): Seasonal patterns of methane uptake and carbon dioxide release by a temperate woodland soil. Global Biogeochem. Cycles 5: 319-334.

Crill, P.M., P.J. Martikainen, H. Nykanen und J. Silvola (1994): Temperature and $\mathrm{N}$-fertilization effects on methane oxidation in a drained peatland soil. Soil Biol. Biochem. 26, 1331-1339.

Christensen, S. und J.M. TIEDJE (1990): Brief and vigorous $\mathrm{N}_{2} \mathrm{O}$ production by soil at spring thaw. J. Soil Sci. 41, 1-4.

Cruzen, P.J. und D.H. EnHALT (1977): Effects of nitrogen fertilizers and combustion on the stratospheric ozone layer. Ambio 6, 112-117.

Crutzen, P.J., L.E. Heidt, J.P. Krasnec, W.H. Polloc und W. Seiler (1979): Biomass burning as a source of atmospheric gases $\mathrm{CO}, \mathrm{H}_{2}, \mathrm{~N}_{2} \mathrm{O}, \mathrm{CH}_{3} \mathrm{Cl}$ and COS. Nature 282, 253-256.

CRUTZEN, P.J. (1991): Methane's sinks and sources. Nature 350, 380-381.

Czepiel, P.M., P.M. CRILl und R.C. HARRIES (1995): Environmental factors influencing the variability of methane oxidation in temperate zone soils. J. Geophys. Res. 100 D5, 9359-9364.

DAVIDSON, E.A. (1991): Fluxes of nitrous oxide and nitric oxide from terrestrial ecosystems. pp. 219-235 in J.E. Rogers and W.B. Whitman (eds.): Microbial production and consumption of greenhouse gases: Methane, nitrogen oxides and halomethanes. American Society for Microbiolgy, Washington, D.C..

DAVIDSON, E.A. (1992): Sources of nitric oxide and nitrous oxide following wetting of dry soil. Soil Sci. Soc. Am. J. 56, 95-102. 
Davidson, E.A., P.A. Matson, P.M. Vitousek, R. Riley, K. Dunkin, G. GarciaMENDEZ und J.M. MAASS (1993): Processes regulating soil emissions of NO and $\mathrm{N}_{2} \mathrm{O}$ in a seasonally dry tropical forest. Ecology 74, 130-139.

Denmead, O.T., J.R. Freney und J.R. Simpson (1979): Studies of nitrous oxide emission from a grass sward. Soil Sci. Soc. Am. J. 43, 726-728.

DoBBIE, K.E. und K.A. SMITH (1996): Comparison of methane oxidation rates in woodland, arable and set aside soils. Soil Biol. Biochem. 28, 1357-1365.

Dobbie, K.E., K.A. Smith, A. Prieme, S. Christensen, A. Degorska und P. Orlansky (1996): Effect of land use on the rate of methane uptake by surface soils in Northern Europe. Atmos. Environ. 30, 1005-1011.

DörR, H., L. KaRTRufF und I. LeVIN (1993): Soil texture parameterization of the methane uptake in aerated soils. Chemosphere 26, 697-713.

Do NGOC, Q. (1975): Untersuchungen zur Dynamik des $\mathrm{NH}_{4}-$ und $\mathrm{NO}_{3}$-Stickstoffs in einer Parabraunerde, einem Podsol und einer Braunerde-Rendzina und zur $\mathrm{N}$-Aufnahme des Maises nach Pflugfurche und Minimalbodenbearbeitung. Diss. Hohenheim.

Dunfield, P.F., R. KNowles, R. Dumont und T.R. Moore (1993): Methane production and consumption in temperate and subarctic peat soils Response to temperature and pH. Soil Biol. Biochem. 25, 321-326.

DUNFIELD, P.F. und R. KNOWLES (1995): Kinetics of inhibition of methane oxidation by nitrate, nitrite, and ammonium in a humisol. Applied Microbiology 61, 8, 3129-3135.

Duxbury, J. M., D.R. Bouldin, R.E. TerRy und R.L. TAte (1982): Emissions of nitrous oxide from soils. Nature, 289, 462-464.

DuXbury, J. M., L.A. HARPER und A. R. Mosier. (1993): Contribution of agroecosystems to global climate change. pp. 1-18. In: Agricultural Ecosystem Effects on Trace Gases and Global Climate Change, ASA Special Publication Number 55, Madison.

DUXBURY, J. M. (1984): Factors affecting nitrous oxide production by denitrification in soils. In: V.P. Aneja (ed.): Environmental impact of natural emission. Air Pollution Control Association. Pittsburgh, 82-99.

EATON, L.J. und D.G. PARIQUIN (1989): Denitrification in lowbush blueberry soils. Can. J. Soil Sci. 69, 303-312.

EICHNER, M.J. (1990): Nitrous oxide emissions from fertilized soils: Summary of available data. J. Environ. Qual. 19, 272-280.

Engel,T. und A. MANGstL (1988): Entwicklung des $\mathrm{N}_{\min }$-Gehaltes im Herbst und Winter auf Praxisschlägen. Mitt. Ges. Pflanzenbauwiss. 1, 12-14. 
ERIKSEn, A.B. und L. Holtem-HaRtwig (1993): Emission spectrometry for direct measurements of nitrous oxide and dinitrogen from soil. Soil Sci. Soc. Am. J. 57, 738-742.

FINDLAY, W.I. und D.J. McKENNEY (1979): Direct measurement of nitrous oxide flux from soil. Can. J. Soil Sci. 59: 413-421.

Firestone, M.K., R.B. FiRestone und J.M. TiedJe (1979): Nitric oxide as an intermediate in denitrification: evidence from nitrogen-13 isotope exchange. Biochemical and Biophysical Research Communications 91, 1, 10-16.

Firestone, M.K., R.B. Firestone und J.M. TiedJe (1980): Nitrous oxide from soil denitrification - Factors controlling its biological production. Science 208, 749-751.

FIRESTONE, M.K. und E.A. DAVIDSON (1989): Microbiological basis of $\mathrm{NO}$ and $\mathrm{N}_{2} \mathrm{O}$ production and consumption in soil, in: Exchange of trace gases between terrestrial ecosystems and the atmosphere, eds. M.O. Andreae and D.S. Schimel, pp. 7-21.

Fischer, J., S. Behnke, K. Westrum und E. Przemeck (1992): Auswirkungen einer Stickstoffreihendüngung zu Kartoffeln auf Ertrag, Qualität und Rest- $\mathrm{N}_{\min }$ im Vergleich zur breitwürfigen Düngung. VDLUFA-Schriftenreihe 34, Kongreßband, 511-514.

FlessA, H. und F. BeEse (1995): Effects of sugarbeet residues on soil redox potential and nitrous oxide emission. Soil Sci. Soc. Am. J., 59, 1044-1051.

FlessA, H., P. Dörsch und F. BeEse (1995). Seasonal variation of $\mathrm{N}_{2} \mathrm{O}$ and $\mathrm{CH}_{4}$ fluxes in differently managed soils in southern Germany. J. Geophys. Res. 100: 23115 - 23124.

Flessa, H., W. Pfau, P. Dörsch und F. Beese (1996): The influence of nitrate and ammonium fertilization on $\mathrm{N}_{2} \mathrm{O}$ release and $\mathrm{CH}_{4}$ uptake of a well-drained topsoil. Z. Pflanzenernähr. Bodenk. 159, 499-503.

Flessa, H., f. Beese, R. Brumme, E. Przemeck, E. Priesack, R. Ruser, R. Teepe, F. SchmäDEKE, C. HABERBosch, R. Stenger, T. LiCKFEtT und R. Schilling (1998): Freisetzung und Verbrauch der Spurengase $\mathrm{N}_{2} \mathrm{O}$ und $\mathrm{CH}_{4}$ beim Anbau nachwachsender Rohstoffe. Abschlußbericht zum Verbundprojekt der Deutschen Bundesstiftung Umwelt, Osnabrück (im Druck).

FUNK, R. (1993): Verlagerung und Abbau von Nitrat in tieferen Bodenschichten süddeutscher Ackerstandorte. Diss. TU-München.

GERMON, J.C. (1985): Microbiology of denitrification and other processes involving the reduction of oxygenated nitrous compounds. In: H.L. GoltERMANN (ed.): Denitrification in the nitrogen cycle. Nato Conference Series I (9), 3146. 
Gisı, U., R. Schenker, R. Schulin, F.X. Stadelmann und H. Sticher (1990): Bodenökologie, Georg Thieme Verlag Stuttgart, New York.

GranLI, T. und O.C. BockMAN (1994): Nitrous oxide from agriculture. Norwegian Journal of Agricultural Sciences, Norsk Hydro Research Centre, Porsgunn Norway.

GRofFMAN, P.M. (1991): Ecology of nitrification and denitrification in soil evaluated at scales relevant to atmospheric chemistry. pp. 201-217. In: J. Rogers und W. Whitman (Eds.), Microbial production and consumption of greenhouse gases: Methane, nitrous oxide and halomethanes. American Society for Microbiology, Washington, USA.

Hansen, S., J.E. Maehlum und L.R. Bakken (1993): $\mathrm{N}_{2} \mathrm{O}$ and $\mathrm{CH}_{4}$ fluxes in soil influenced by fertilization and tractor traffic. Soil Biol. Biochem. 25,5, 621630.

HANTSCHEL, R.E., H. Flessa und F. BeEse (1994): An automated microcosm system for studying soil ecological processes. Soil Sci. Soc. Am. J. 58, 401-404.

HarRIS, R.C., D.I. Sebacher und F.P. DAY (1992): Methane flux in the Great Dismal Swamp. Nature 297, 673-674.

HARTGE, K.H. und R. HORN (1991): Einführung in die Bodenphysik. Ferdinand EnkeVerlag, Stuttgart.

HARTGE, K.H. und R. HORN (1992): Die physikalische Untersuchung von Böden. Ferdinand Enke-Verlag, Stuttgart.

HAUCK, R.D., S.W. MELSTED und P.E. YANKWICH (1958): Use of N-isotope distribution in nitrogen gas in a study of denitrification. Soil Sci. 86, 287-291.

Healy, R. W., R. G. Striegl, T. F. Russell, G. L. Hutchinson und G.P. Livingston (1996): Numerical evaluation of static-chamber measurements of soilatmosphere gas exchange: identification of physical process. Soil Sci. Soc. Am. J., 60, 740-747.

Heinemeyer, O., K. Haider und A.R. Mosier (1988): Einfluß kontrollierter Wasserzufuhr zu mit Mais bepflanztem Boden auf $\mathrm{N}_{2} \mathrm{O}$ - und $\mathrm{N}_{2}$ - Freisetzung aus ${ }^{15} \mathrm{~N}$-Nitrat; Kontinuierliche Redoxpotentialmessung in verschiedenen Bodentiefen. Mitt. Dt. Bodenkundl. Ges. 56, 283-288.

HEYDER, D. (1993): Nitratverlagerung, Wasserhaushalt und Denitrifikationspotential in mächtigen Lößdecken und einem Tonband bei unterschiedlicher Bewirtschaftung. Bonner Bodenkd. Abhandl. 10, $171 \mathrm{~S}$.

HEYER, J. (1993): Methan, Studienprogramm Landwirtschaft der EnqueteKommission Schutz der Erdatmosphäre. Economica Verlag, Bonn. 
Houba, V.J.G., J. Novozamsky, A.W.M. Huygregts und J. VAN deR Lee (1986): Comparison of soil extraction by $0,01 \mathrm{M} \mathrm{CaCl}_{2}$ by EUF and by some conventional extraction procedures. Plant and Soil, 96, 433-437.

HÜTSCH, B.W., C.P. WEBSTER und D.S. POWLSON (1994): Methane oxidation in soil as affected by land use, soil $\mathrm{pH}$ and $\mathrm{N}$ fertilization. Soil Biol. Biochem. 26, 1613-1622.

HydRo AGRI (1993): Faustzahlen für Landwirtschaft und Gartenbau. Verlagsunion Agrar, 12. überarbeitete, ergänzte und erweiterte Auflage.

HYNES, R.K. und R. KNOWLES (1980): Denitrification, nitrogen fixation and nitrification in continuous flow laboratory soil columns. Can. J. Soil Science 60, 355-363.

INGRAHAM, J.L. (1981): Microbiology and genetics of denitrifiers, in: Denitrification, nitrification and atmospheric nitrous oxide. John Wiley and sons New York, 45-53.

IPCC (1994): Radiative forcing of climate change. The 1994 report of the scientific assessment working group of IPCC. Summary for policymakers. WMO/UNEP, Geneva, Switzerland.

IPCC (1995): IPCC second assessment. Climate change 1995. A report of the intergovernmental panel on climate change. WMO/UNEP, Geneva, Switzerland.

IPCC (1996): Climate change 1995. Scientific and technical analyses of impacts, adaptations and mitigation. Contribution of working group II to the second assessment report of the intergovernmental panel on climate change. University press, Cambridge.

JANSSON, S.L. (1958): Tracer studies on nitrogen transformations in soil with special attention to mineralisation/immobilisation relationships. Kungl. Landbr. Ann. 24, 101.

Kalser, E.-A., K. Kohrs, M. KüCKe, E. Schnug, O. Heinemeyer und J.C. Munch (1997): Nitrous oxide release from arable soil: Importance of $\mathrm{N}$-fertilization, crops and temporal variation. Soil Biol. Biochem. (eingereicht).

KaltSCHMitT, M. und G.A. ReINHARD (1997): Nachwachsende Energieträger, Grundlagen, Verfahren, ökologische Bilanzierung. Vieweg-Verlag, Braunschweig/Wiesbaden.

KAMP, T und H. STEINDL (1997): $\mathrm{N}_{2} \mathrm{O}$-emissions from agriculture in a warmer climate How will soils react on a climate change. pp 297-302. In: K.H. Becker und P. Wiesen (Eds.): Proceedings of the $7^{\text {th }}$ International workshop on nitrous oxide emissions, Cologne, Germany. 
Keller, M., W.A. Kaplan, S.C. Wofsy und J.M. DaCosta (1988): Emissions of nitrous oxide from tropical forest soils: Response to fertilization with ammonium, nitrate, and phosphate. J. Geophys. Res. 93, 1600-1604.

Keller, M., M.E. Mitre und R.R. Stallard (1990): Consumption of atmospheric methane in soils of central Panama: Effects of agricultural developement. Global Biogeochem. Cycles 4, 21-27.

KhALIL, M.A.K. und R.A. RASMussen (1990): Atmospheric methane: recent global trends. Environ. Sci. Technol. 24, 549-553.

KING, G.M. und A.P.S. AdAMSON (1992): Effects of temperature on methane consumption in a forest soil and in pure cultures of the methanotroph. Appl. Environ. Microbiol. 58, 2758-2763.

Klemedtsson, L., B.H. Svensson und T. Rosswall (1988): Relationships between soil moisture content and nitrous oxide production during nitrification and denitrification. Biol. Fertil. Soils 6, 106-111.

KÖBRICH, D. (1995): Die Emission von Distickstoffoxid $\left(\mathrm{N}_{2} \mathrm{O}\right)$ aus einer ackerbaulich genutzten Fläche -Quantifizierung und Modellierung - Diss. TU Braunschweig.

Kölle, W., P. Werner, O. Strebel und J. Böttcher (1983): Denitrifikation in einem reduzierten Grundwasserleiter. Vom Wasser 61, 125-147.

Kölle, W., O. Strebel und J. BötTCheR (1985): Formation of sulfate by microbial denitrification in a reducing aquifer. Wat. Supply 3, 35-40.

Kölle, W., O. Strebel und J. Böttcher (1990): Reduced sulfur compounds in sandy aquifers and their interactions with groundwater. Proc. of the Dresden Symposium: Groundwater Monitoring and Management. IAHS Publ. 173, 23-30.

Kralova, M., P.H. Masscheleyn, C.W. Lindau und W.H. Patrick JR. (1992): Production of dinitrogen and nitrous oxide in soil suspensions as affected by redoxpotential. Water Air Soil Pollution 61, 37-45.

LANGel, R., A. REINEKING und R. Well (1995): On-line equilibrating of ${ }^{15} \mathrm{~N}$-gases using gas discharge. Isotopes in Environmental and Health Studies 31, 305-306.

Lessard, R., P. Rochette, E. Topp, E. Pattey und R.L. Desjardins (1994): Methane and carbon dioxide fluxes from poorly drained adjacent cultivated and forest sites. Can J. Soil Sci. 74, 139-146.

LICKFETT, T. (1993): Auswirkungen verminderter Produktionsintensität in zwei Rapsfruchtfolgen auf Elemente des N-Haushaltes im System Boden Pflanze. Diss. Uni Göttingen. 
Lickfett, T. und E. PrzemeCK (1997): Auswirkungen von Extensivierungsmaßnahmen auf den Stickstoffhaushalt. pp. 91-125. In: GEROWITT, B. und WILDENHAYN, M. (Hrsg.): Ökologische und ökonomische Auswirkungen von Extensivierungsmaßnahmen im Ackerbau - Ergebnisse des Göttinger INTEX-Projektes 1990 - 1994. Golze Verlag, Göttingen.

LIND, A.M. (1985): Soil air concentration of nitrous oxide over 3 years of field experiments with animal manure and inorganic nitrogen fertilizer. Tidsskr. Planteavl 89, 331-340.

LINN, D.M. und J.W. DoRAN (1984): Effect of water-filled pore space on carbon dioxide and nitrous oxide production in tilled and nontilled soils. Soil Sci. Soc. Am. J. 48, 1267-1272.

LiPSChUltZ, F., O.C. ZafiRIOU, S.C. Wofsy, M.B. McEloy, F.W. ValoIS und S.W. WATSON (1981): Production of $\mathrm{NO}$ and $\mathrm{N}_{2} \mathrm{O}$ by soil nitrifying bacteria. Nature 294, 641-643.

Loftfield, N., H. Flessa, J. Augustin and F. Beese (1997): Automated gas chromatographic system for rapid analysis of the atmospheric trace gases methane, carbon dioxide, and nitrous oxide. J. Environ. Qual., 26/2, 560-564.

MAIDL, F.C. und G. FISCHBECK (1987): Nitratgehalte tieferer Bodenschichten bei unterschiedlichen Fruchtfolgen auf intensiv genutzten Ackerbaustandorten. Z. Pflanzenernähr. Bodenk. 150, 213-219.

MALINOWSKY, P. und J.C.G. OTTOW (1985): Ökologische Bedingungen der Denitrifikation bei Pilzen. Landwirtsch. Forsch. 38, 30-34.

Marrero, T.R. und E.A. Masson (1972): Gaseous diffusion coefficients. J. Phys. Chem. Ref. Data 1, 3-118.

Matson, P.A., P.M. Vitousek, G.P. Livingstone und N.A. Swanberg (1990): Sources of variation in nitrous oxide flux from amazonian ecosystems. J. Geophys. Res. 95, 16789-16798.

McDonald, J.A., U. SkiBA, L.J. Sheppard, K.J. HaRgreaves, K.A.Smith und D. FOWLER (1996): Soil environmental variables affecting the flux of methane from a range of forests, moorlands and agricultural soils. Biogeochemistry 34, 113-132.

Melin, J. und H. Nommik (1983): Denitrification measurements in intact soil cores. Acta Agriculturae Scandinavica 33, 145-151.

MOCHOGE, B. und F. BEESE (1983): The behaviour of nitrogen fertilizer in neutral and acid loess soils. 1. Transport and transformation of nitrogen. $Z$. Pflanzenernähr. Bodenkd. 146, 504-515. 
Mosier, A.R. und G.L. Hutchinson (1981): Nitrous oxide emissions from cropped fields. J. Environ. Qual. 10(2), 169-173.

Mosier, A.R., W.J. PARTON und G.L. HutChinson (1983): Modelling nitrous oxide evolution from cropped and native soils. Environ. Biogeochem. Ecol. Bull. 35, 229-241.

MosieR, A.R., W.D. GuenzI und E.E. SchweIzer (1986): Soil losses of dinitrogen and nitrous oxide from irrigated crops in northeastern Colorado. Soil Sci. Soc. Am. J. 50, 344-348.

Mosier, A.R., D. Schimel, D. Valentine, K. Bronson und W. Patron (1991): Methane and nitrous oxide fluxes in native, fertilized and cultivated grasslands. Nature 350, 330-332.

MuLVANEY, R.L. und L.T. KuRTZ (1984): Evolution of dinitrogen and nitrous oxide from nitrogen-15 fertilized soil cores subjected to wetting and drying cycles. Soil Sci. Soc. Am. J. 48, 596-602.

MummeY, D.L., J.L. SMith und H. Bolton (1994): Nitrous oxide flux from a shrubsteppe ecosystem: sources and regulation. Soil Biol. Biochem. 26, 2, 279-286.

MuNCH, J.C. und J.C.G. OTTOW (1987): Einfluß von Bodenfeuchte und Sauerstoff auf die Lachgasbildung denitrifizierender Bakterien. Mitt. Dt. Bodenkundl. Ges. 55/II, 505-510.

MuRAKAMI, T. und K. KumaZAWA (1987): Measurement of denitrification products in soil by the acetylene inhibition method. Soil Sci. Plant Nutr. 33, 225-234.

OJima, D.S., D.W. Valentine, A.R. Mosier, W.J. Parton, und D.S. Schimel (1993): Effect of land use change on methane oxidation in temperate forest and grassland soils. Chemosphere, 26, 675-685.

Papen, H. und H. Rennenberg (1990): Microbial processes involved in emissions of radiatively important trace gases. In: Transactions $14^{\text {th }}$ International Congress of Soil Science, Kyoto. Vol. 2, 232-237.

PARTON, W.J., A.R. Mosier und D.S. Schimel (1988): Rates ant pathways of nitrous oxide production in a shortgrass steppe. Biogeochemistry 6: 45-58.

PAYNE, W.J. (1995): Diversity of denitrifiers and their enzymes, in: Denitrification in the nitrogen cycle. Plenum press, New York, 47-65.

PenNington, P.C. und R.C. ElLIS (1993): Autotrophic and heterotrophic nitrification in acid forest and native grassland soils. Soil Biol. Biochem. 25, 10, 1399-1408.

PostmA, D. (1990): Kinetics of nitrate reduction by detrital Fe(II)-silicates. Geochim Cosmochim. Acta 54, 903-908. 
Postma, D., C. Boesen, H. Kristiansen und F. LARSEN (1991): Nitrate reduction in an unconfirmed aquifer: Water chemistry, reduction processes, and geochemical modelling. Water Res. 27, 2027-2045.

POtTER, C.S., E.A. DAVIDSON und L.V. VeRCHOt (1996): Estimation of global biogeochemical controls and seasonality in soil methane consumption. Chemosphere 32, 2219-2246.

Pratt, P.E., W.W. Jones und V.E. HUnSAKER (1972): Nitrate in deep soil profiles in relation to fertilizer rates and leaching volume. J. Environ. Qual. 1, 97-102.

Prieme, A., S. Christensen, K.E. Dobbie und K.A. Smith (1997): Slow increase in rate of methane oxidation in soils with time, following land use change from arable agriculture to woodland. Soil Biol. Biochem. 29, 1165-1172.

PRZEMECK, E. und T. LiCKFETT (1992): Untersuchungen zum Nährstoffhaushalt der Böden. In: Feldversuche zur Extensivierung im Ackerbau. 104. VDLUFAKongress Göttingen, Exkursionsführer, 76-83.

RACKWITZ, R. (1996): Modelluntersuchungen zur Stickstoffdynamik in Schwarzerden mit unterschiedlicher Düngungsgeschichte. Diss. TU München.

ROBERTSON, G.P. (1989): Nitrification and denitrification in humid tropical ecosystems: Potential controls on nitrogen retention. pp. 55-68 in J. Procter (ed.): Mineral nutrients in tropical forests and savanna ecosystems. Blackwell Scientific Publications, Oxford.

RoLLAND, W. (1995): Organotrophe und chemolithoautotrophe Denitrifikation in der ungesättigten Zone - Messung und Simulation -. Diss Bonn.

Rolston, D.E., D.L. HofFMAnN und D.W. ToY (1978): Field measurement of denitrification. 1. Flux of nitrogen and nitrous oxide. Soil Sci. Soc. Am. J. 42, 863-869.

Rolston, D.E., A.N. Sharpley, D.W. Toy und F.E. Broadbend (1982): Field measurement of denitrification; 3. Rates during irrigation cycles. Soil Sci. Soc. Am. J. 46, 289-296.

Rudaz, A.O., E.A. DAvidson und M.K. FIRESTONE (1991): Sources of nitrous oxide production following wetting of dry soil, FEMS Microbiology Ecology 85, 117-124.

Ruser, R., H. Flessa und F. Beese (1996): Plant-type and N-fertilizer dependent variation in nitrous oxide emissions. pp. 539-542. In: K.H. Becker und P. Wiesen (Eds.): Proceedings of the $7^{\text {th }}$ International workshop on nitrous oxide emissions, Cologne, Germany. 
Ruser, R., H. Flessa, R. Schilling, H. Steindl und F. Beese (1998): Effects of soil compaction and fertilization on $\mathrm{N}_{2} \mathrm{O}$ and $\mathrm{CH}_{4}$ fluxes in potato fields. Soil Sci. Soc. Am. J., (zur Publikation eingereicht).

Ruz-JEREZ, B.E., R.E. WHITE und P.R. BALL (1994): Long-term measurement of denitrification in three contrasting pastures grazed by sheep. Soil Biol. Biochem. 26, 1, 29-39.

RYDEN, J.C., L.J. LUND und D.D. FOCHT (1980): Direct measurement of denitrification loss from soil. II. Development and application of field methods. Soil Sci. Soc. Am. J. 43, 110-118.

RYDEN, J.C. und L.J. LUND (1980): Nitrous oxide evolution from irrigated land. J. Environ. Qual. 9, 387-393.

Sahrawat, K.L. und D.R. KeEney (1996): Nitrous oxide emissions from soils. Adv. Soil Sci. 4, 103-148.

SAUERBECK, D. und K. ISERMANN (1993): Herkunft und Strategien zur Verminderung der Emission von klimarelevanten Spurengasen - Bereich Land- und Forstwirtschaft. 7. Mühlheimer wassertechnisches Seminar, IWWSchriftenreihe, Rhein.-Westfäl. Inst. f. Wasserchemie und Wassertechnol., Mühlheim / Ruhr, 1-27.

Schachtschabel, P., H.-P. Blume, G. Brümmer, K.-H. Hartge und U. SCHWERTMANN (1989): Scheffer/Schachtschabel: Lehrbuch der Bodenkunde. Auflage 12, Ferdinand Enke Verlag, Stuttgart.

SCHEFFER, B. (1993): Nitratauswaschung - Nach Rapsanbau steigen die Nitratwerte. Landwirtschaftsblatt Weser-Ems, 36, 20-21.

SCHIMEL, J.P., E.A. Holland und D. VAlentine (1993): Controls on methane flux from terrestrial ecosystems. pp. 167-182. In: Agricultural Ecosystem Effects on Trace Gases and Global Climate Change, ASA Special Publication Number 55, Madison.

SCHLEGEL, H.G. (1985): Allgemeine Mikrobiologie. 6. überarbeitete Auflage, Thieme Verlag, Stuttgart.

Schlichting, E. und H.P. Blume (1966): Bodenkundliches Praktikum. Paul Parey, Hamburg und Berlin.

SChlichting, E., H.P. Blume und K. StAR (1995): Bodenkundliches Praktikum. Blackwell Wissenschaftsverlag, Berlin.

SCHLIE, P. (1989): Hydrologie des Grundwasserwerkes Stegemühle. Diss., Göttinger Arb. Geol. Paläont., 43. 
SCHNEIDER, U. (1991): Messungen von Denitrifikations- und Nitratauswaschungsverlusten in einem landwirtschaftlich genutzten Wassereinzugsgebiet. Diss., Bonn.

SCHNELL, S. und G.M. KING (1994): Mechanistic analysis of ammonium inhibition of atmospheric methane consumption in forest soils. Appl. Environm. Microb. 60, 3514-2321.

SCHULTE-KelLINGHAUS, S. (1987): Über die Denitrifikation in der ungesättigten Zone mächtiger Lösse und grundwassernaher Standorte. Diss. Bonn.

Schulz, H., W. Seiler und H. RenNenberg (1990): Soil and land use related sources and sinks of methane $\left(\mathrm{CH}_{4}\right)$ in the context of the global methane budget. Soils and the Greenhouse Effect, A.F. Bouwman, Editor; John Wiley and Sons, pp. 270-285.

SCHUSTER, M. und R. KONRAD (1992): Metabolism of nitric oxide and nitrous oxide during nitrification and denitrification in soil at different incubation conditions. FEMS Microbiol. Ecol. 101, 133-143.

SEILER, W. und R. ConRAD (1981): Mikrobielle Bildung von $\mathrm{N}_{2} \mathrm{O}$ (Distickstoffoxid) aus Mineraldüngern - ein Umweltproblem?, forum mikrobiologie 6, 322-328.

SHARP, B. (1991): Nitrous oxide emissions from soils. Expected modifications as a consequence of current trends in land use. CEC Report EUR 13473 EN, Brussels.

SHEPHERD, M.F., S. BARZETTI und D.R. HASTIE (1991): The production of atmospheric $\mathrm{NO}_{x}$ and $\mathrm{N}_{2} \mathrm{O}$ from a fertilized agricultural soil. Atmos. Environ. 25A, 19611969.

SKIBA, U., K.A. SMITH und D. FowLER (1993): Nitrification and denitrification as sources of nitric oxide and nitrous oxide in a sandy loam soil. Soil Biol. Biochem. 25, 11, 1527-1536.

SMITH, K.S. und J.R.M. ARAH (1990): Losses of nitrogen by denitrification and emissions of nitrogen oxides from soils. Proc. Fert. Soc. London, No 299.

Sommerfeld, R.A., A.R. Moisier und R.C. Musselmann (1993): $\mathrm{CO}_{2}, \mathrm{CH}_{4}$ and $\mathrm{N}_{2} \mathrm{O}-$ flux through a Wyoming snowpack and implications for global budgets. Nature 361, 140-142.

Staley, T.E., W.H. CAskey und D.G. Boyer (1990): Soil denitrification and nitrification potentials during the growing season relative to tillage. Soil Sci. Soc. Am. J. 54, 1602-1608.

StATISTISCHES BundeSAMt (1997); Wiesbaden, Deutschland 
Steudler, P.A., R.D. Bowden, J.M. Melillo und J.D. Aber (1989): Influence of nitrogen fertilization on methane uptake in temperate forest soils. Nature 341, 314-316.

Steudler, P.A., R.D. Jones, M.S. CAstro, J.M. Mellilo und D.L. LeWis (1996): Microbial controls of methane oxidation in temperate forests and agricultural soils. P. 69-84. In: Microbiology of Atmospheric Trace Gases, edited by J.C. Murrell and D.P. Kelly, NATO ASI Series, Springer-Verlag Berlin, p.39.

StRIEGEL, R.G. (1993): Diffusional limits to the consumption of atmospheric methane by soils. Chemosphere 26, 715-720.

Stumm, W. und J.J. MoRgan (1981): Aquatic chemistry. $2^{\text {nd }}$ ed. Wiley-Interscience, New York.

TeIWES, K. (1997): Standortbedingungen. pp. 25-34 In: GeRowitT, B. und WILDENHAYN, M. (Hrsg.): Ökologische und ökonomische Auswirkungen von Extensivierungsmaßnahmen im Ackerbau - Ergebnisse des Göttinger INTEX-Projektes 1990 - 1994. Golze Verlag, Göttingen.

ThOMPSON, A.M. und R.J. CICERONE (1996): Possible pertubation to atmospheric CO, $\mathrm{CH}_{4}$, and $\mathrm{OH}$. J. Geophys. Res 91, 10853-10864.

TORBERT, H.A. und C.W. WoOD (1992): Effects of soil compaction and water-filled pore space on soil microbial activity and $\mathrm{N}$ losses. Comm. Soil Sci. Plant Anal. 23, 1321-1331.

Tortoso, A.C. und G.L. HutChinson (1990): Contribution of autotrophic and heterotrophic nitrifiers to soil $\mathrm{NO}$ and $\mathrm{N}_{2} \mathrm{O}$ emissions. Appl. Environ. Microbiol. 56, 6, 1799-1805.

Trudell , M.R., R.W. Gillham und J.A. Cherry (1996): An in-situ study of the occurence and rate of denitrification in a shallow unconfined sand aquifer. Journal of Hydrology 83, 251-268.

Tyler, S.C., P.M. CRILl und G.W. Brailsford (1994): C13/C14 fractionation of methane during oxidation in a temperate forest. Geochimica et Cosmochimica Acta 58, 6, 1625-1633.

UBA (1992): Jahresbericht 1992 des Umweltbundesamtes: 10. Schutz der Erdatmosphäre, 149-152.

UBA (1993): Ökologische Bilanz von Rapsölmethylester (RME) als Ersatz von Dieselkraftstoff (Ökobilanz Raps). Umweltbundesamt, Berlin.

U.S. Environmental Protection Agency (1989): Policy options for stabilizing global climate. p. 20. In D.A. Lashof and D.A. Tirpak (ed.): Draft report to Congress. USEPA Office of Policy, Planning and Evaluation, Washington, DC. 
Van Beek, C.G.E.M., H. Boukes, D. Van Riusbergen und R. Straatmann (1988): The threat of the Netherlands waterworks by nitrate in the abstracted groundwater, as demonstrated on the well field Vierlingsbeek. Water Supply 6, 313-318.

Van Kessel, C., J. Pennock, und R.E. Farrell (1993): Seasonal variations in denitrification and nitrous oxide evolution at the landscape scale. Soil Sci. Soc. Am. J. 57, 988-995.

Van Cleemput, O., J. Van Hoorde und A. Vermoesen (1992): Emission of $\mathrm{N}_{2} \mathrm{O}$ under different cropping systems. pp. 20-21. In E. Francois, K. Pithan und N. Bartiaux-Thill (eds.): Proc. Whorkshop Cost 814: Nitrogen cycling and leaching in cool and wet regions of Europe. CRA, Gembloux.

VDLUFA (1991): Methodenbuch, Band I. Die Untersuchung von Böden. VDLUFAVerlag, Darmstadt.

Voss, G. (1985): Zur Nitratverlagerung in mächtigen Lössdecken des Vorgebirges bei Bonn. Diss. Bonn.

Watson, R.T., L.G. Meira Filho, E. Sanhueza und A. Janetos (1992): Greenhouse gases: Sources and sinks. In: Climate change, The IPCC Scientific Assessment (Houghton J.T., Jenkins, G.J., Ephraums, J.J., (eds.)) Cambridge Univ. Press, Cambridge, UK, 1-41.

WeIER, K.L. und J.W. GILlIAM (1986): Effect of acidity on denitrification and nitrous oxide evolution from atlantic coastal-plain soils. Soil Sci. Soc. of Am. J. 50, 1202-1205.

Weier, K.L., J.W. Doran, J.F. PoWer und D.T. Walters (1993 a): Denitrification and the dinitrogen/nitrous oxide ratio as affected by soil water, available carbon and nitrate. Soil Sci. Soc. Am. J. 57, 66-72.

WeieR, K.L., I.C. MAcRae und R.J.K. Myers (1993 b): Denitrification in a clay soil under pasture and annual crop: estimation of potential losses using intact soil cores. Soil Biol. Biochem. 25, 8, 991-997.

WeieR, K.L., I.C. MAcRae und R.J.K. Myers (1993 c): Denitrification in a clay soil under pasture and annual crop: losses from ${ }^{15} \mathrm{~N}$-labelled nitrate in the subsoil in the field using $\mathrm{C}_{2} \mathrm{H}_{2}$ inhibition. Soil Biol. Biochem. 25, 8, 999-1004.

WELL, R. (1993): Denitrifikation im Wurzelraum unterhalb der Ackerkrume. MessMethodik und Vergleich der ${ }^{15} \mathrm{~N}$-Bilanz mit der ${ }^{15} \mathrm{~N}$-Gasfreisetzungs-Methode. Diss Göttingen.

Well, R., K.-W. Becker, R. Langel, B. Meyer und A. Reineking (1998): Continuous flow equilibration from mass spectrometric analysis of dinitrogen emissions. Soil Sci. Soc. Am. J. (eingereicht). 
Westermann, R.L. und L.T. KURTZ (1973): Priming effect of ${ }^{15} \mathrm{~N}$-labelled fertilizers on soil nitrogen in field experiments. Soil Sci. Soc. Am. Proc. 37, 725-727.

Westermann, R.L. und T.C. TUCKER (1974): Effect of salts and salts plus nitrogen15-labelled ammonium chloride on mineralization of soil nitrogen, nitrification, and immobilization. Soil Sci. Soc. Am. Proc. 38, 602-605.

Whalen, S.C., W.S. ReEBURGH und K.A. SANDBECK (1990): Rapid methane oxidation in a landfill cover. Soil Applied and Environmental Microbiology 56, 34053411.

WISOTZKY, F. (1994): Untersuchungen zur Pyritoxidation in Sedimenten des Rheinischen Braunkohlereviers und deren Auswirkungen auf die Chemie des Grundwassers. Bes. Mitteil. dt. Gewässerkdl. Jahrbuch 58, S. 153.

WOLFENSBERGER, U. und F. DINKEL (1997): Beurteilung nachwachsender Rohstoffe in der Schweiz in den Jahren 1993-1996. Eidgenössische Forschungsanstalt für Agrarwissenschaften und Landtechnik, Tänikon, Schweiz.

WoOdS, L.E., C.V. Cole, L.K. PoRTER und D.C. Coleman (1987): Transformation of added and indigenous nitrogen in gnotobiotic soil: a comment on the priming effect. Soil Biol. Biochem. 19, 6, 673-678.

ZÖTTL, H. (1960): Die Mineralstickstoffanlieferung in Fichten- und Kiefernbeständen Bayerns. Forstw. Cbl. 79, 221. 


\section{Lebenslauf}

\begin{tabular}{|c|c|c|}
\hline Name & Frank Schmädeke & \\
\hline Geburtsdatum & 23.01 .65 & \\
\hline Geburtsort & Nienburg/Weser & \\
\hline \multirow[t]{3}{*}{ Schulausbildung } & $1971-1975$ & Grundschule Heemsen \\
\hline & $1975-1985$ & Gymnasium Nienburg \\
\hline & & Abschluß: $\quad \underline{\text { Abitur }}$ \\
\hline \multirow[t]{2}{*}{ Berufsausbildung } & $1985-1987$ & landwirtschaftl. Lehre \\
\hline & & Abschluß: Landwirt \\
\hline Grundwehrdienst & $1987-1988$ & \\
\hline \multirow[t]{3}{*}{ Studium } & $1988-1994$ & Universität Göttingen \\
\hline & & Agrarwissenschaften \\
\hline & & Fachrichtung Pflanzenbau \\
\hline \multirow[t]{2}{*}{ Diplomarbeit } & 05.1994 & $\begin{array}{l}\text { Institut für Boden- } \\
\text { wissenschaften }\end{array}$ \\
\hline & & Abschluß: Dipl. Ing. agr. \\
\hline \multicolumn{3}{|l|}{ Wissenschaftl. Tätigkeit } \\
\hline Wissenschaftl. Hilfskraft & $09.1994-12.1994$ & Institut f. Agrikulturchemie \\
\hline Promotion & seit 01.1995 & Institut f. Agrikulturchemie \\
\hline \multirow[t]{3}{*}{ Zusätzl. Tätigkeiten } & 28.03. - 12.04.1991 & freiwilliges Praktikum im \\
\hline & & $\begin{array}{l}\text { Pflanzenschutzamt der LWK } \\
\text { (Bezirksstelle Nienburg) }\end{array}$ \\
\hline & 4.02. - 03.04.1992 & $\begin{array}{l}\text { Werkstudent im Forschungs- } \\
\text { zentrum Monheim (Bayer AG) }\end{array}$ \\
\hline
\end{tabular}




\section{Danksagung}

An dieser Stelle möchte ich allen Menschen, die mir bei der Anfertigung dieser Arbeit mit Rat, Tat und moralischer Unterstützung zur Seite gestanden haben, bedanken. Mein besonderer Dank gilt:

Herrn Prof. Dr. Eberhard Przemeck für die Überlassung des interessanten Themas. Ich danke inm weiterhin für die große Freiheit, die er mir zur Bearbeitung des Projektes überließ, sowie für die Korrektur der Arbeit,

Herrn Professor Dr. Friedrich Beese für die Möglichkeit, am Institut für Bodenkunde und Waldernährung einen großen Teil meiner Laboranalysen durchzuführen sowie für die freundliche Übernahme des Korreferats,

den Herren Dr. Thomas Lickfett, Dr. Rainer Brumme und Dr. Heiner Flessa für die gute und freundschaftliche Zusammenarbeit, für ihre ständige Gesprächs- und Hilfsbereitschaft sowie für die vielen methodischen Anregungen,

Frau Gabi Dormann und Frau Kirsten Essig, die nicht nur zu jeder Zeit zur Stelle waren, wenn es galt, große Analysenmengen zu bewältigen, sondern immer ein angenehmes Arbeitsklima verbreiteten und jederzeit ein offenes Ohr für große und kleine Probleme hatten - Herzlichen Dank !

Herrn Professor Dr. Brunk Meyer sowie allen Mitarbeitern des Institutes für Bodenwissenschaften für die ständige Gesprächs- und Hilfsbereitschaft,

meinen Mitdoktoranden Herrn Rainer Ruser und Herrn Robert Teepe im Verbundprojekt "Nachwachsende Rohstoffe" für die wissenschaftlich effektive und freundschaftliche Zusammenarbeit,

den Herren Dr. Werner Borken, Dr. Jörg Priess und Lars Szwecz für die inhaltliche und praktische Unterstützung in Sachen Spurengasanalytik,

dem Forschungs- und Studienzentrum Landwirtschaft und Umwelt für die Bereitstellung der Versuchsflächen,

Frau Michaela Meyer und den Herren Detlev Kopf, Dr. Michael Wildenhayn, Edgar Schreiber und Horst Schröder, auf die ich mich immer verlassen konnte, wenn es galt, die ackerbaulichen Maßnahmen zu planen oder durchzuführen.

Für die kritische Durchsicht des Manuskripts danke ich Frau Gudula Kreykenbohm, Herrn Dr. Reinhard Well, Frau Susanne Küster und Herrn Dr. Jörg Küster.

Ganz besonderer Dank gilt meinen Eltern, die mir mit moralischer Unterstützung und außerwissenschaftlicher Hilfe in allen Phasen der Promotion zur Seite standen.

Diese Arbeit wurde mit Mitteln der Deutsche Bundesstiftung Umwelt im Rahmen des Verbundprojektes „klimarelevante Spurengasflüsse beim Anbau nachwachsender Rohstoffe" gefördert. 